FEB. 04, 2016

\title{
Synthetic Access to Atomically Dispersed Metals in Metal-Organic Frameworks via a Combined Atomic-Layer-Deposition-in-MOF and Metal-Exchange Approach
}

Rachel C. Klet, ${ }^{\dagger}$ Timothy C. Wang, ${ }^{\dagger}$ Laura E. Fernandez, ${ }^{\S}$ Donald G. Truhlar, ${ }^{*, \S}$ Joseph T. Hupp, ${ }^{*, \dagger}$ Omar K. Farha, ${ }^{*, \dagger, \perp}$

${ }^{\dagger}$ Department of Chemistry, Northwestern University, Evanston, IL 60208, United States $\perp$ Department of Chemistry, Faculty of Science, King Abdulaziz University, Jeddah, Saudi Arabia

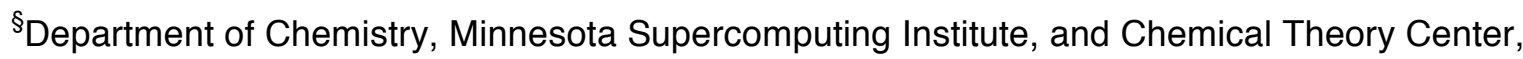
University of Minnesota, Minneapolis, Minnesota 55455, United States

\section{Table of Contents}

\begin{tabular}{|l|l|}
\hline Materials and Methods & $\mathrm{S}-2$ \\
\hline Instrumentation & $\mathrm{S}-2$ \\
\hline Modified ALD procedure for Zn-AIM & $\mathrm{S}-2$ \\
\hline Zn/Zr $_{6}$ Ratio for AIM at T $=100-140^{\circ} \mathrm{C}$ & $\mathrm{S}-3$ \\
\hline DRIFTS comparing Zn-AIM and NU-1000 & $\mathrm{S}-3$ \\
\hline ICP-OES Analysis & $\mathrm{S}-3$ \\
\hline Metal Exchange Data for Cu salts for 3, 6, and $20 \mathrm{~h}$ & $\mathrm{~S}-4$ \\
\hline TGA traces for AIM-ME-Cu, AIM-ME-Ni, and AIM-ME-Co & $\mathrm{S}-5$ \\
\hline SEM-EDX for AIM-ME-Cu & $\mathrm{S}-5$ \\
\hline Metal Exchange Data for Ni and Co salts for 3, 6, and $20 \mathrm{~h}$ & $\mathrm{~S}-6$ \\
\hline SEM-EDX for AIM-ME-Ni & $\mathrm{S}-6$ \\
\hline SEM-EDX and AIM-ME-Co & $\mathrm{S}-7$ \\
\hline DRIFTS spectra for AIM-ME-Cu, AIM-ME-Ni, and AIM-ME-Co & $\mathrm{S}-7$ \\
\hline Computational Details of Zn-AIM Calculations & $\mathrm{S}-8$ \\
\hline Quantum Mechanical Results for Zn Coordination & $\mathrm{S}-12$ \\
\hline Cartesian Coordinates for Computational Structures & $\mathrm{S}-13$ \\
\hline Reference & $\mathrm{S}-127$ \\
\hline
\end{tabular}




\section{Materials and Methods.}

Methanol (Macron), $\mathrm{NiCl}_{2} \cdot 6 \mathrm{H}_{2} \mathrm{O}$ (Strem), $\mathrm{Ni}\left(\mathrm{NO}_{3}\right)_{2} \cdot 6 \mathrm{H}_{2} \mathrm{O}, \mathrm{CuCl}_{2} \cdot 2 \mathrm{H}_{2} \mathrm{O}, \mathrm{CoCl}_{2} \cdot 6 \mathrm{H}_{2} \mathrm{O}$, $\mathrm{CuBr}_{2}, \mathrm{Cu}\left(\mathrm{NO}_{3}\right)_{2} \cdot 2.5 \mathrm{H}_{2} \mathrm{O}$ (Sigma-Aldrich) were used as received. $\mathrm{NU}-1000^{1}$ was synthesized according to a method reported in the literature. Metal salt stock solutions were prepared in $50 \mathrm{~mL}$ volumetric flasks.

\section{Instrumentation.}

Activation of MOF samples was performed on a Micromeritics SmartVacPrep (Micromeritics, Norcross, GA, USA) and $\mathrm{N}_{2}$ adsorption isotherms were measured on a Micromeritics Tristar II 3020 at $77 \mathrm{~K}$ with the temperature held constant using liquid $\mathrm{N}_{2}$ bath. Powder X-ray diffraction (PXRD) patterns were recorded on a Rigaku ATXG diffractometer equipped with an $18 \mathrm{~kW}$ Cu rotating anode, MLO monochromator, and a high-count-rate scintillation detector. Atomic layer deposition was carried out in a Savanah S100 system (Ultratech Cambridge Nanotech) under $\mathrm{N}_{2}$. Scanning electron microscopy (SEM) images and energy-dispersive X-ray spectroscopy (EDS) mapping were recorded on a Hitachi SU8030 SEM. Thermogravimetric analyses (TGA) were performed on a TGA/DCS 1 system (Mettler-Toledo AG, Schwerzenbach, Switzerland), which runs on a PC with STAR ${ }^{\mathrm{e}}$ software. Samples were heated from $25^{\circ} \mathrm{C}$ to $700^{\circ} \mathrm{C}$ at $10{ }^{\circ} \mathrm{C} /$ minute rate under flowing $\mathrm{N}_{2}$. Inductively Coupled Plasma-Optical Emission Spectroscopy (ICP-OES) was collected on a Thermo iCAP 7600.

\section{Modified ALD procedure for Zn-AIM}

NU-1000 (40 mg) was loaded into a home-built stainless steel powder holder and subsequently placed into an ALD reactor at the target temperature. NU-1000 was allowed to equilibrate in the reactor for $0.5 \mathrm{~h}$ prior to the ALD deposition. The ALD reactions were carried out utilizing the following timing sequence (time in $s$ ): $t_{1}-t_{2}-t_{3}$, where $t_{1}$ is the precursor pulse time, $t_{2}$ the precursor exposure time (i.e., the time where the precursor is in contact with $\mathrm{NU}-1000$ without pumping), and $t_{3}$ the $\mathrm{N}_{2}$ purge time. $\mathrm{ZnEt}_{2}$ was deposited at temperatures between 100-140 ${ }^{\circ} \mathrm{C}$ utilizing sixteen 1-240-240 sequences, and the $\mathrm{N}_{2}$ flow was held constant at $15 \mathrm{sccm}$. After the exposure sequences of $\mathrm{ZnEt}_{2}$, the reactor was purged with $\mathrm{N}_{2}$ for additional 10 min, and another $t_{1}-t_{2}-t_{3}$ sequence with water was carried out. Water was dosed into the reactor utilizing sixteen 0.015-120-120 sequences, and the $\mathrm{N}_{2}$ flow was held constant at $15 \mathrm{sccm}$. Based on ICP-OES analysis of $\mathrm{ZnEt}_{2}$ AIM performed at temperatures between 100-140 ${ }^{\circ} \mathrm{C}$, we determined the optimal temperature for deposition of $\mathrm{ZnEt}_{2}$ in NU-1000 to be 110 ${ }^{\circ} \mathrm{C}$. 
$\mathrm{Zn} / \mathrm{Zr}_{6}$ Ratio for AIM at $\mathrm{T}=100-140^{\circ} \mathrm{C}$

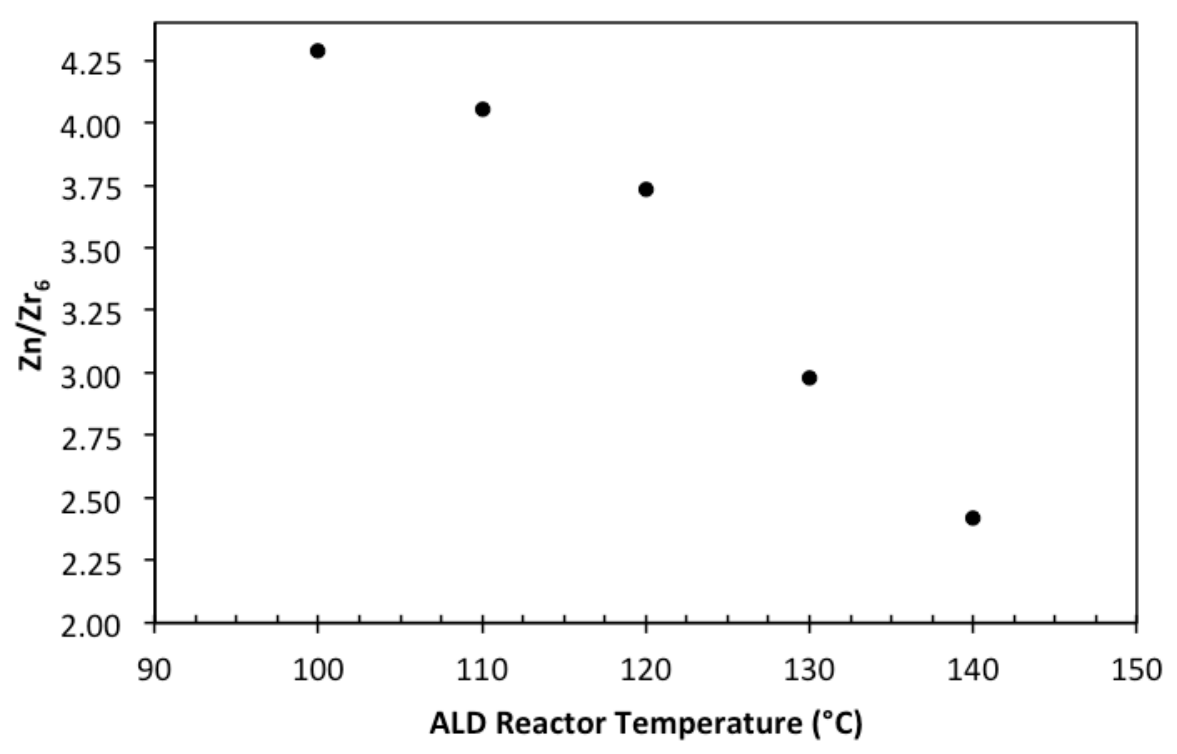

Figure S1. Plot of $Z n / Z r_{6}$ ratio in AIM samples of $N U-1000$ as a function of ALD reactor temperature.
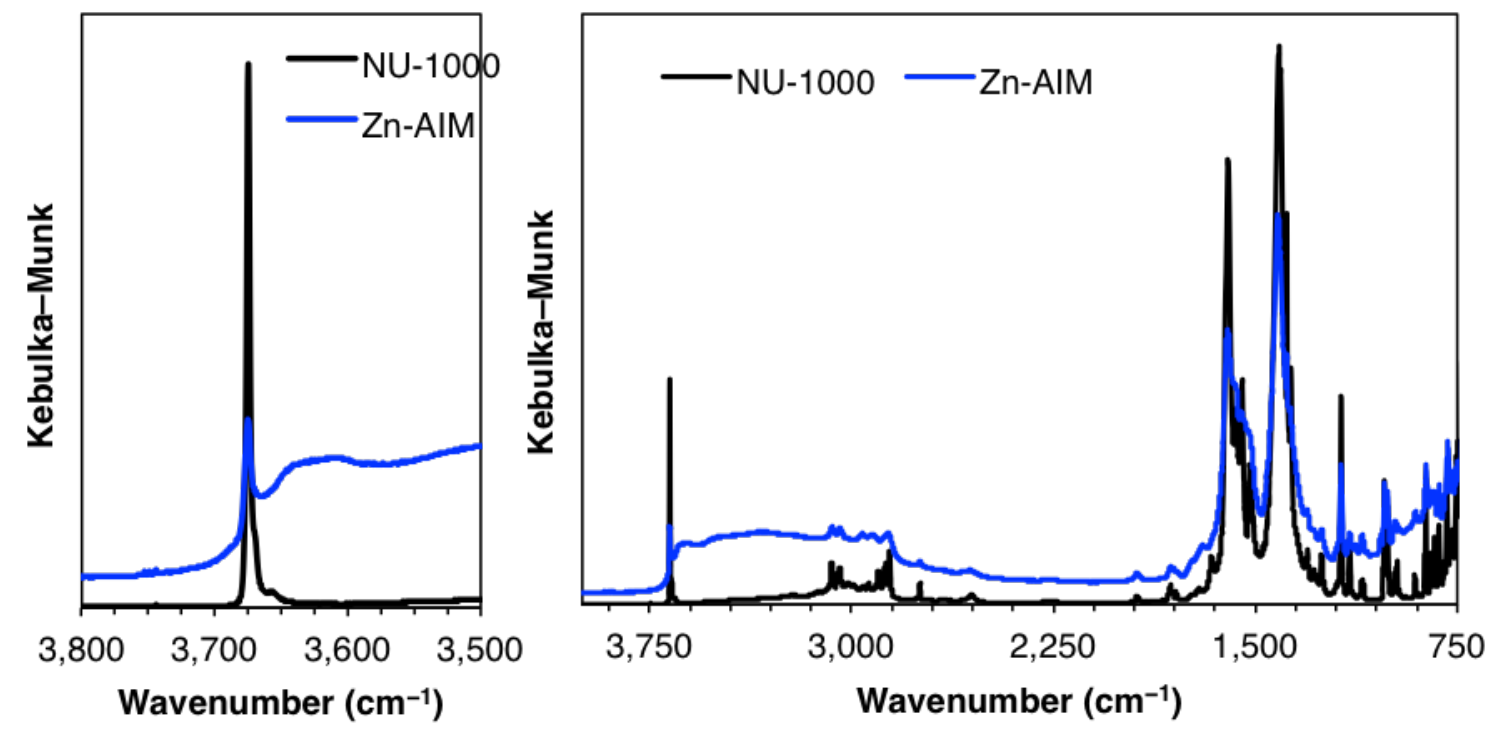

Figure S2. DRIFTS spectra of NU-1000 (black) and Zn-AIM (blue). Full spectra (right) and $-\mathrm{OH}$ region from $3500-3800 \mathrm{~cm}^{-1}$ (left).

\section{ICP-OES Analysis.}

Approximately $1 \mathrm{mg}$ of sample was digested in $1 \mathrm{~mL}$ of piranha solution $\left(1: 330 \% \mathrm{H}_{2} \mathrm{O}_{2}\right.$ / conc. $\mathrm{H}_{2} \mathrm{SO}_{4}$ ), and irradiated in a microwave oven at $150{ }^{\circ} \mathrm{C}$ for $5 \mathrm{~min}$. The colorless solution was then diluted to $25 \mathrm{~mL}$ with nanopure water and analyzed by ICP-OES. Metal concentrations were calculated from external stock solutions and compared to the known $\mathrm{Zr}$ content of the MOF. 
Table S1. Metal Exchange Data for Cu salts for 3, 6, and $20 \mathrm{~h}$.

\begin{tabular}{|c|c|c|c|c|c|c|}
\hline Cu Salt & $\begin{array}{l}\text { Exchange } \\
\text { Time (h) }\end{array}$ & $\mathrm{Zn} / \mathrm{Zr}_{6}$ & $\mathrm{Cu} / \mathrm{Zr}_{6}$ & $\begin{array}{c}\% \text { Nonstructural } \\
\text { Metal Retained } \\
((\mathrm{Zn}+ \\
\text { Cu }) / 4) \times 100 \%\end{array}$ & $\begin{array}{c}\% \mathrm{Cu} \\
\text { Exchange } \\
(\mathrm{Cu} /(\mathrm{Cu}+ \\
\mathrm{Zn})) \times 100 \%\end{array}$ & $\begin{array}{c}\text { Weighted } \\
\text { Effective } \\
\text { Metal } \\
\text { Exchange }\end{array}$ \\
\hline \multirow{3}{*}{$\mathrm{CuCl}_{2} \cdot 2 \mathrm{H}_{2} \mathrm{O}$} & 3 & 0.64 & 2.76 & 85 & 81 & 0.69 \\
\hline & 6 & 0.04 & 3.63 & 92 & 99 & 0.91 \\
\hline & 20 & 0.02 & 3.65 & 92 & 99 & 0.91 \\
\hline \multirow{3}{*}{$\mathrm{CuBr}_{2}$} & 3 & 0.32 & 3.03 & 84 & 90 & 0.76 \\
\hline & 6 & 0.03 & 3.49 & 88 & 99 & 0.87 \\
\hline & 20 & 0.02 & 3.57 & 90 & 100 & 0.89 \\
\hline \multirow{4}{*}{$\mathrm{Cu}\left(\mathrm{NO}_{3}\right)_{2} \cdot 2.5 \mathrm{H}_{2} \mathrm{O}$} & 3 & 0.54 & 2.79 & 83 & 84 & 0.70 \\
\hline & 6 & 0.17 & 3.40 & 89 & 95 & 0.85 \\
\hline & 20 & 0.11 & 3.60 & 93 & 97 & 0.90 \\
\hline & 44 & 0.09 & 3.88 & 99 & 98 & 0.97 \\
\hline
\end{tabular}

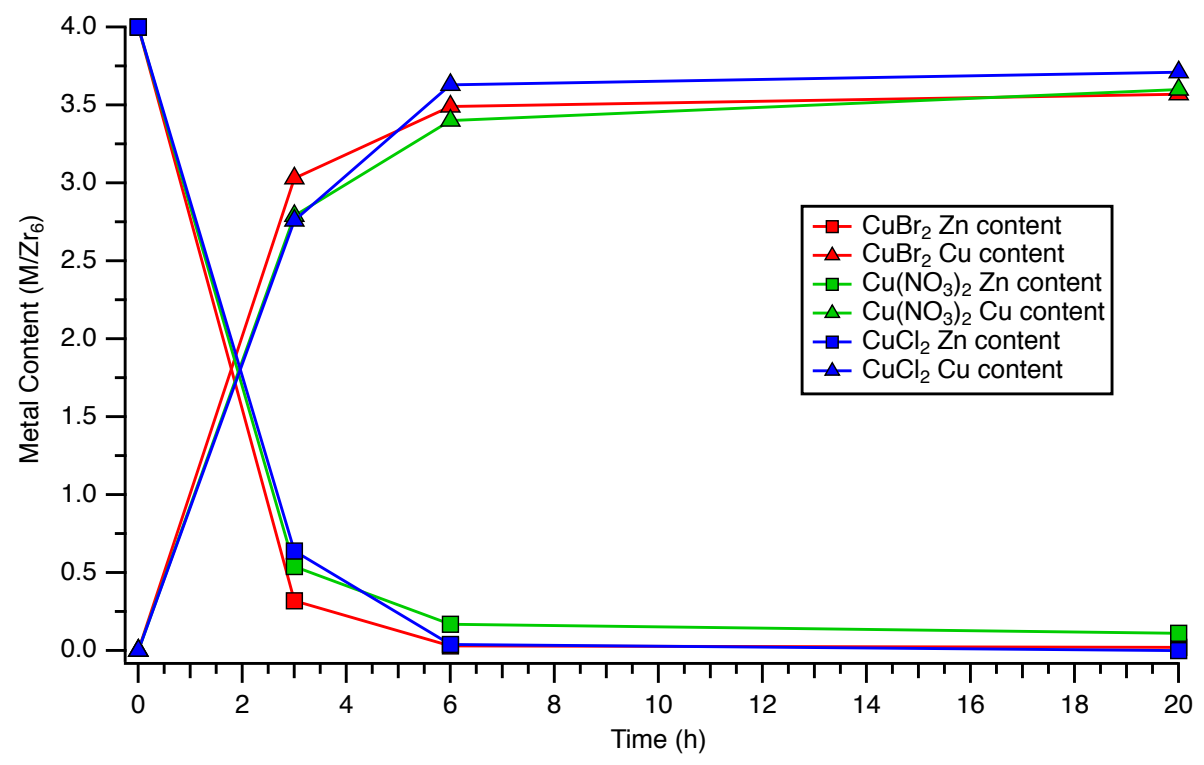

Figure S3. Metal exchange of Zn-AIM with different copper salts showing similar exchange rates. 


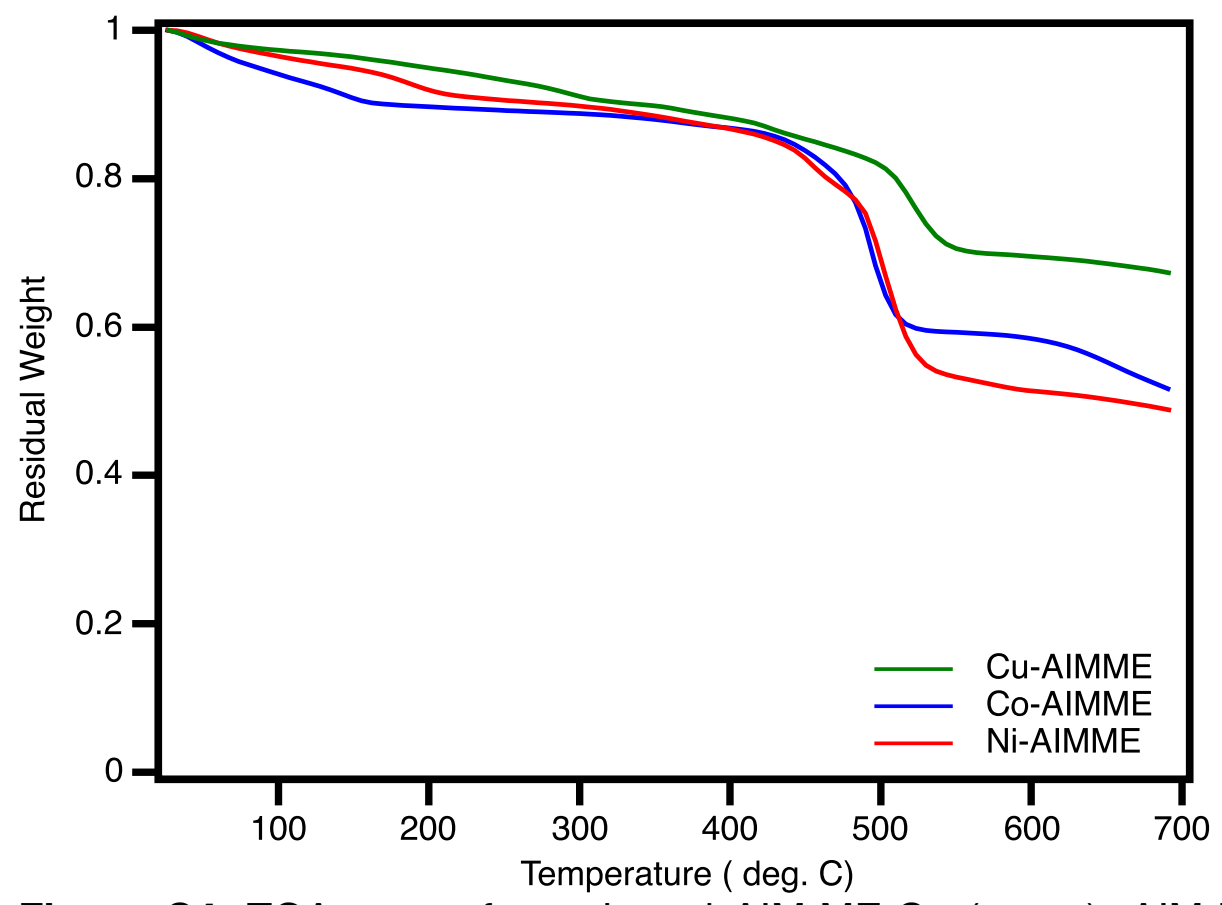

Figure S4. TGA traces for activated AIM-ME-Cu (green), AIM-ME-Ni (red), and AIMME-Co (blue) samples.
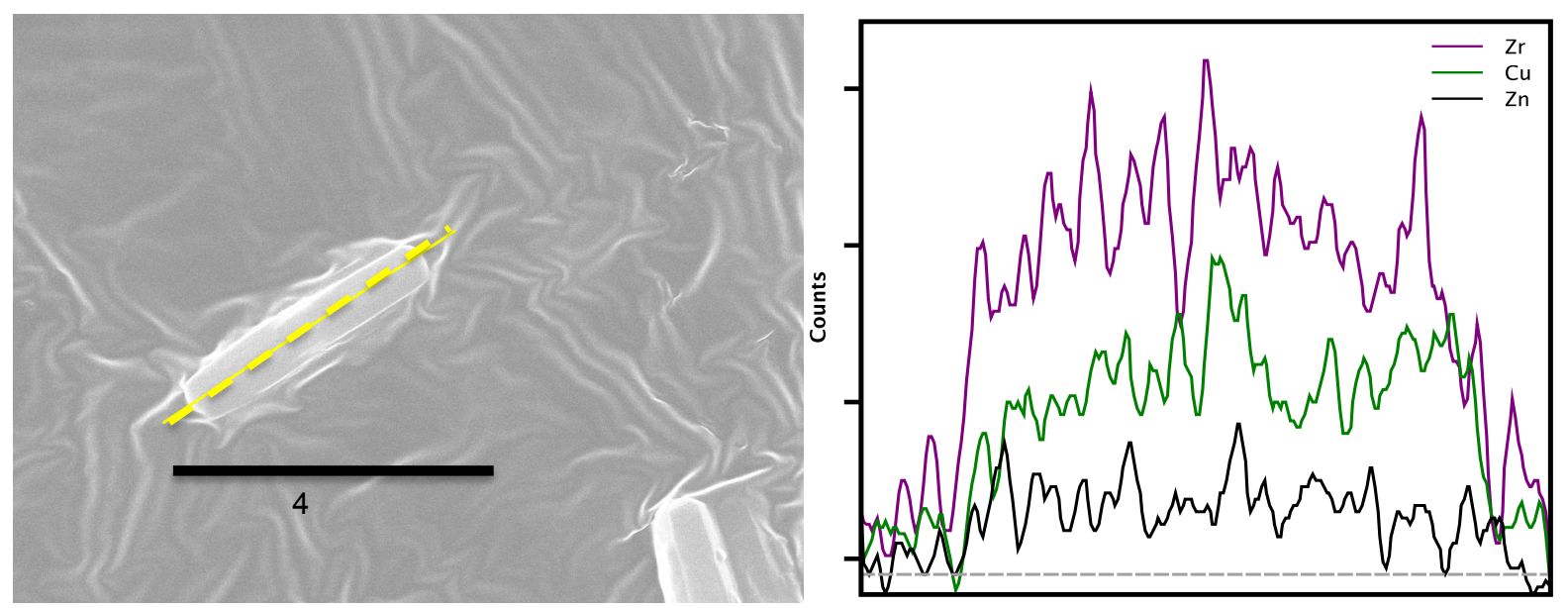

Figure S5. SEM-EDX image and spectrum of AIM-ME-Cu. EDX scan lines for $\mathrm{Zr}, \mathrm{Cu}$, and $\mathrm{Zn}$ are show in purple, green, and black, respectively. The yellow dashed line indicates where the EDX scan was taken. 
Table S2. Metal Exchange Data for $\mathrm{Ni}$ and Co salts for 3, 6, and $20 \mathrm{~h}$

\begin{tabular}{|c|c|c|c|c|c|c|c|}
\hline Metal Salt & $\begin{array}{l}\text { Temp. of } \\
\text { Metal } \\
\text { Exchange }\end{array}$ & $\begin{array}{c}\text { Exchange } \\
\text { Time (h) }\end{array}$ & $\mathrm{Zn} / \mathrm{Zr}_{6}$ & $M / Z r_{6}$ & $\begin{array}{c}\% \\
\text { Nonstructural } \\
\text { Metal } \\
\text { Retained } \\
((\mathrm{Zn}+ \\
\text { M)/4) } \times 100 \% \\
\end{array}$ & $\begin{array}{c}\% M \\
\text { Exchange } \\
(M /(M+ \\
Z n)) \times 100 \%\end{array}$ & $\begin{array}{l}\text { Weighted } \\
\text { Effective } \\
\text { Metal } \\
\text { Exchange }\end{array}$ \\
\hline \multirow{3}{*}{$\mathrm{NiCl}_{2} \cdot 6 \mathrm{H}_{2} \mathrm{O}$} & \multirow{3}{*}{$r t$} & 3 & 1.84 & 1.35 & 80 & 42 & 0.34 \\
\hline & & 6 & 1.64 & 1.50 & 78 & 48 & 0.38 \\
\hline & & 20 & 1.23 & 1.81 & 76 & 59 & 0.45 \\
\hline \multirow{3}{*}{$\mathrm{Ni}\left(\mathrm{NO}_{3}\right)_{2} \cdot 6 \mathrm{H}_{2} \mathrm{O}$} & \multirow{3}{*}{ rt } & 3 & 2.64 & 0.68 & 83 & 21 & 0.17 \\
\hline & & 6 & 2.47 & 0.79 & 81 & 24 & 0.20 \\
\hline & & 20 & 2.08 & 1.03 & 78 & 33 & 0.26 \\
\hline \multirow{3}{*}{$\mathrm{NiCl}_{2} \cdot 6 \mathrm{H}_{2} \mathrm{O}$} & \multirow{3}{*}{$60^{\circ} \mathrm{C}$} & 3 & 0.91 & 1.94 & 71 & 68 & 0.48 \\
\hline & & 6 & 0.81 & 2.16 & 74 & 73 & 0.54 \\
\hline & & 20 & 0.96 & 2.48 & 86 & 72 & 0.62 \\
\hline \multirow{3}{*}{$\mathrm{CoCl}_{2} \cdot 6 \mathrm{H}_{2} \mathrm{O}$} & \multirow{3}{*}{$60^{\circ} \mathrm{C}$} & 3 & 0.64 & 2.43 & 77 & 79 & 0.61 \\
\hline & & 6 & 0.58 & 2.38 & 74 & 80 & 0.59 \\
\hline & & 20 & 0.43 & 3.19 & 91 & 88 & 0.80 \\
\hline
\end{tabular}
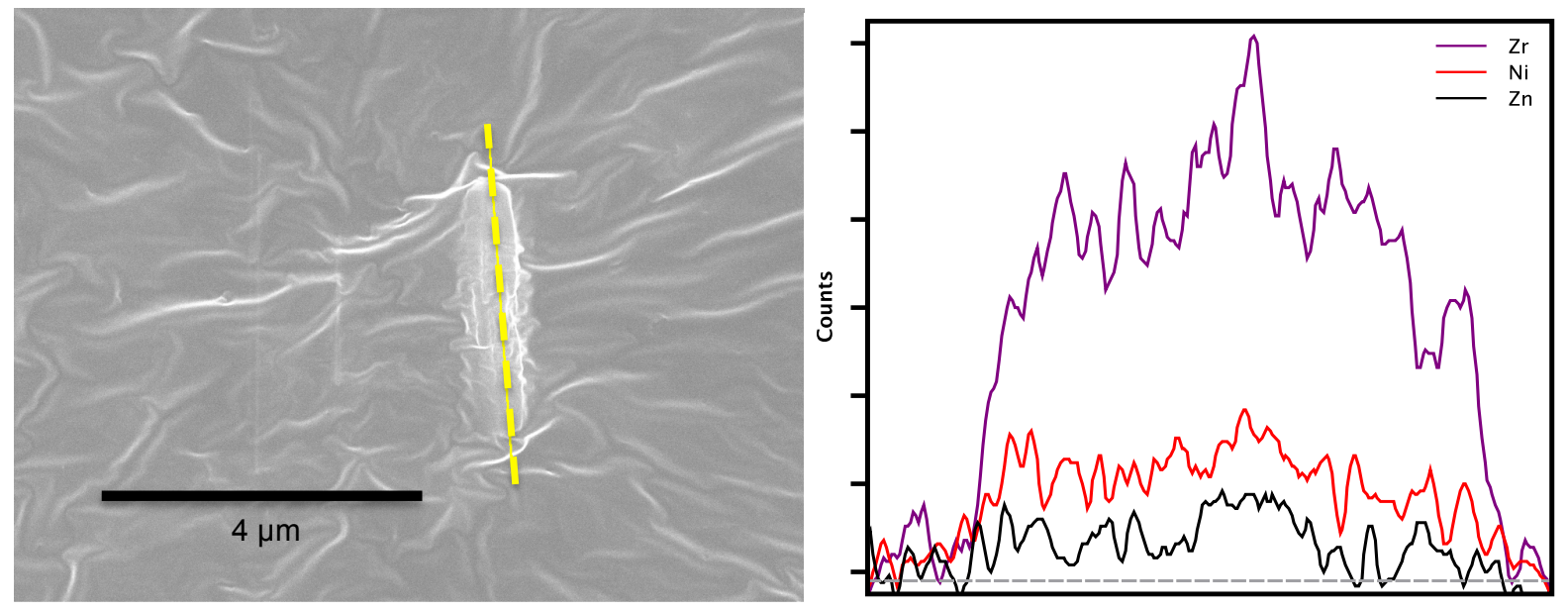

Figure S6. SEM-EDX image and spectrum of AIM-ME-Ni. EDX scan lines for $\mathrm{Zr}$, $\mathrm{Ni}$, and $\mathrm{Zn}$ are show in purple, red, and black, respectively. The yellow dashed line indicates where the EDX scan was taken. 

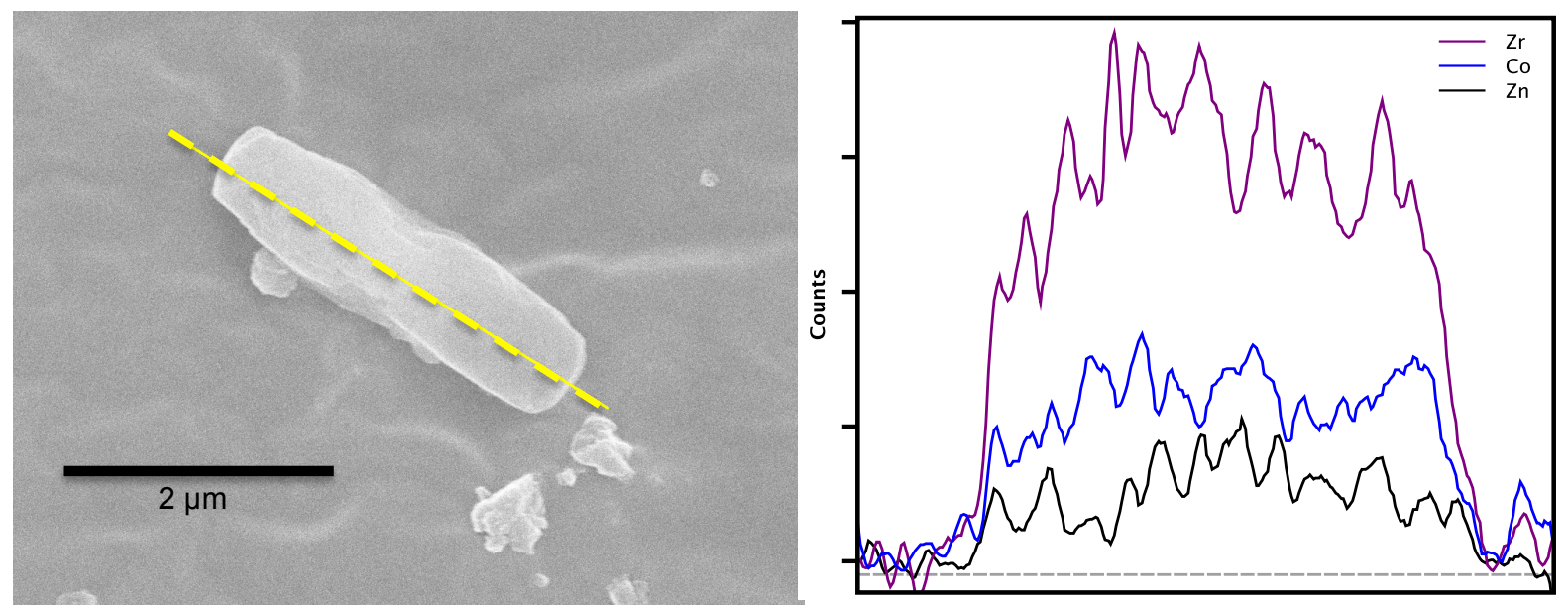

Figure S7. SEM-EDX image and spectrum of AIM-ME-Co. EDX scan lines for $\mathrm{Zr}$, Co, and $\mathrm{Zn}$ are show in purple, blue, and black, respectively. The yellow dashed line indicates where the EDX scan was taken.
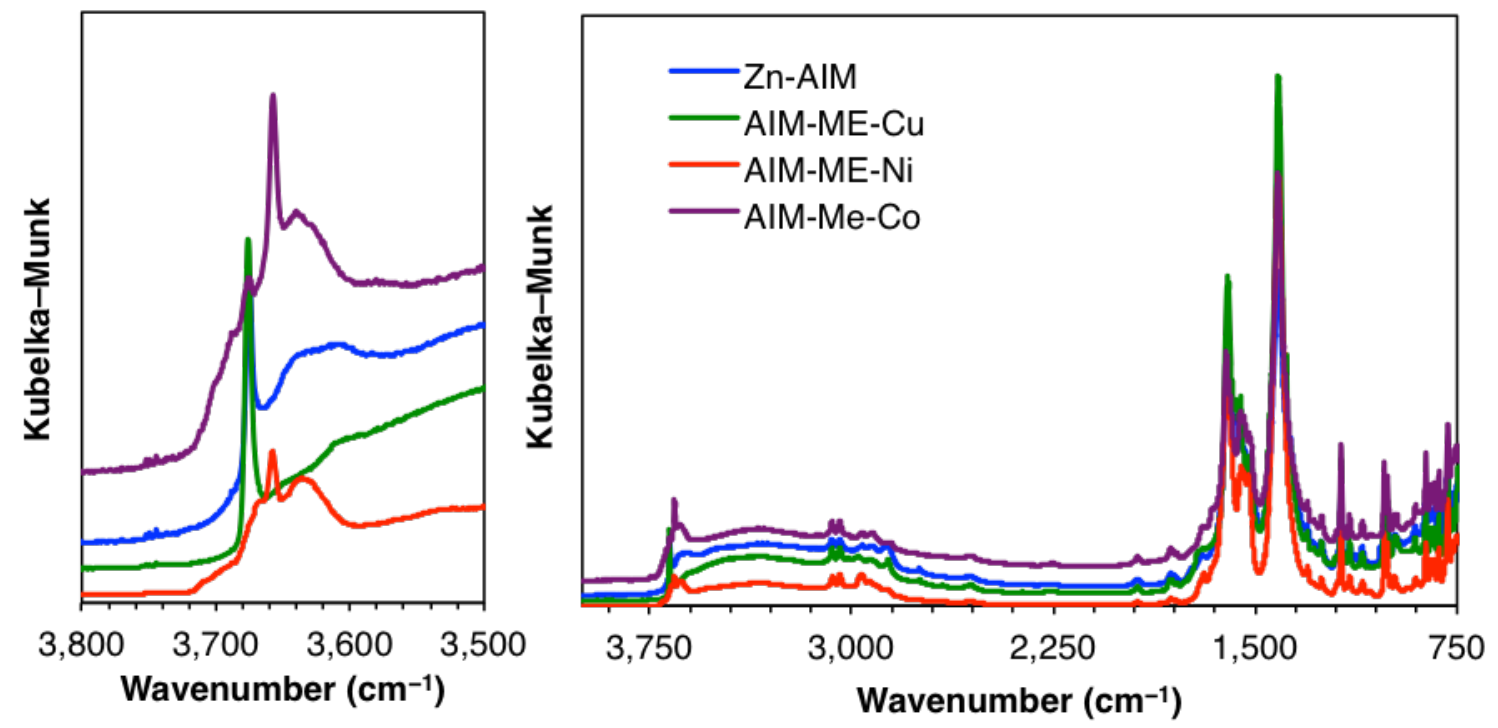

Figure S8. DRIFTS spectra of Zn-AIM (blue), AIM-ME-Cu (green), AIM-ME-Ni (red), and AIM-ME-Co (purple). Full spectra (right) and $-\mathrm{OH}$ region from $3500-3800 \mathrm{~cm}^{-1}$ (left). 


\section{Computational Details of Zn-AIM Calculations}

As seen in Table 1 of the article, the $\mathrm{HB}$ and $\mathrm{H}_{2} \mathrm{O}$ initial binding structures are energetically similar. Furthermore, when the structures of the $\mathrm{HB}$ and $\mathrm{H}_{2} \mathrm{O}$ structures are analyzed more closely, we see that they differ by rotation around the $\mathrm{Zr}-\mathrm{O}$ bond (Figure S9).

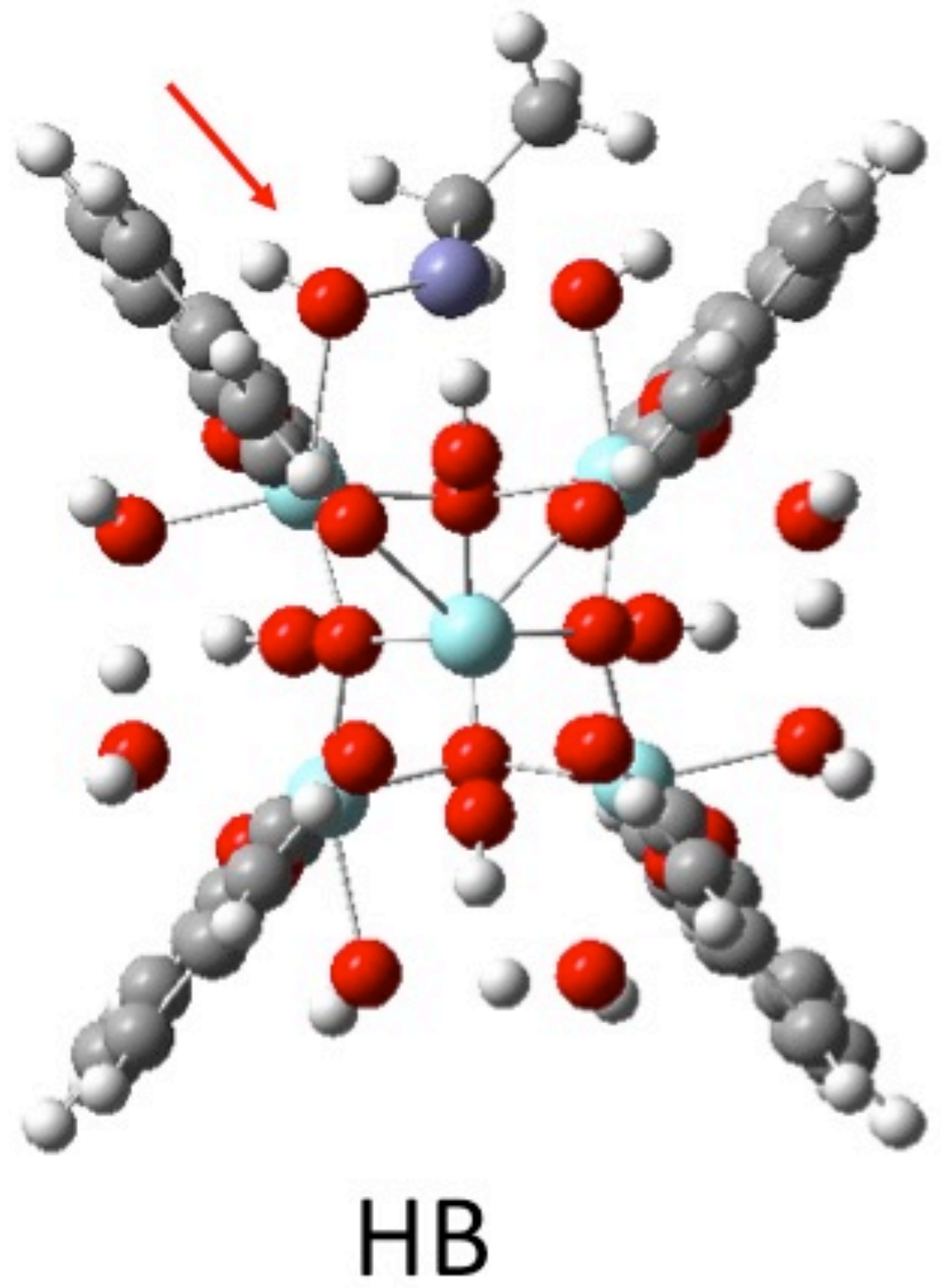




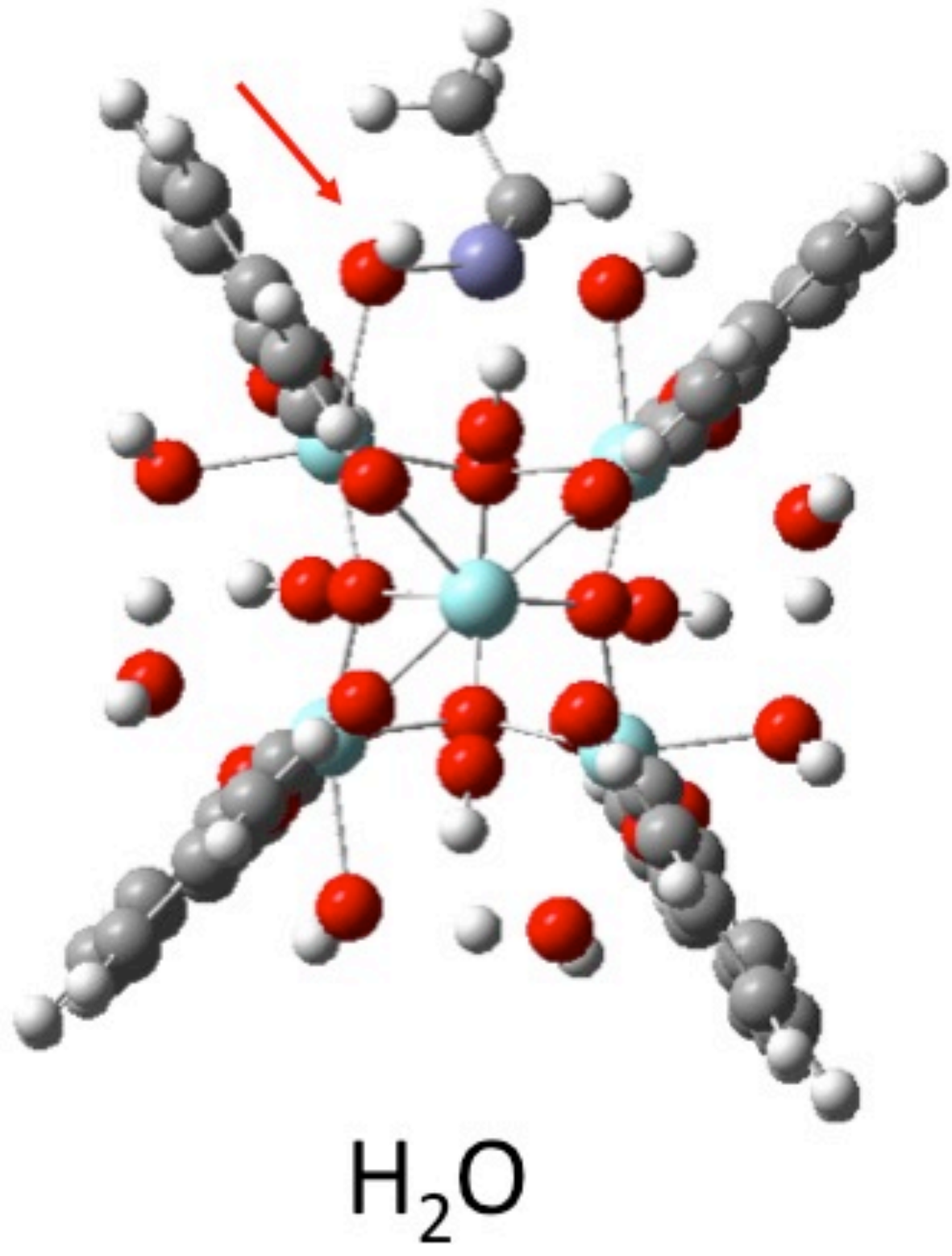

Figure S9. Optimized structures of the $\mathrm{HB}$ and $\mathrm{H}_{2} \mathrm{O}$ initial binding structures with arrows pointing to the $\mathrm{O}$ of the $\mathrm{Zr}-\mathrm{O}$ torsion.

Table 1 and Figure 2 of the article show that the three lowest-energy structures (HB, $\mathrm{H}_{2} \mathrm{O}$, and $\mu \mathrm{OH}$ ) of the $\mathrm{ZnEt}$ adduct are energetically close. Therefore we investigated the addition of a second $\mathrm{ZnEt}_{2}$ precursor to each of these three structures (see Schemes S1-S2 for details of the $\mathrm{HB}$ and $\mu \mathrm{OH}$ ). 


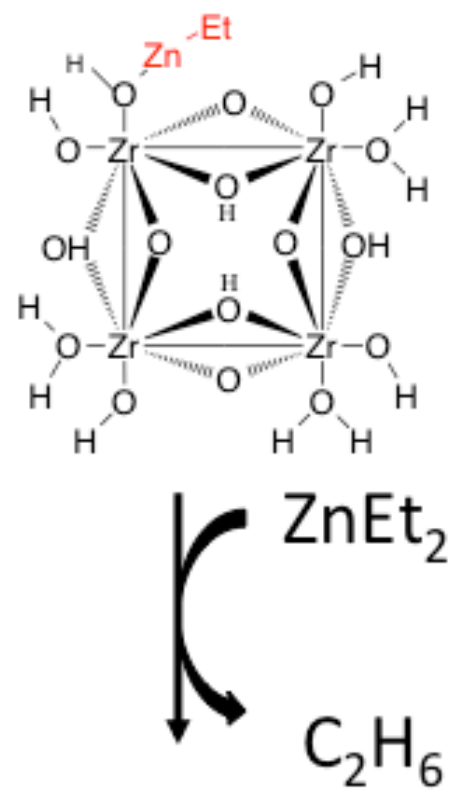

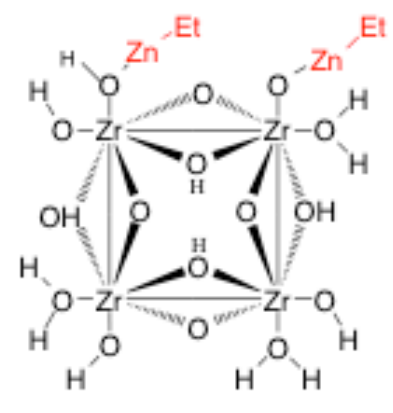

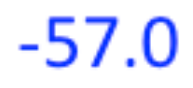

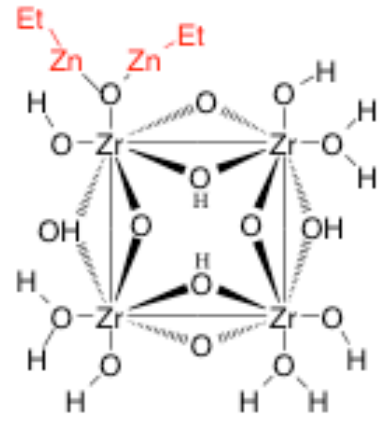

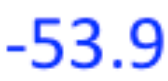

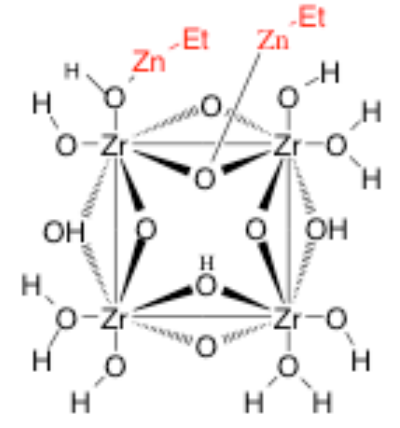

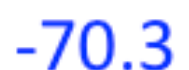

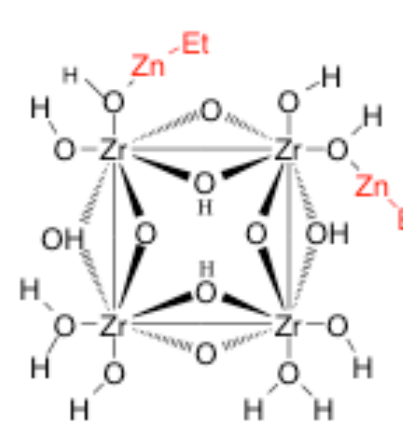

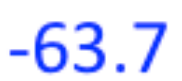

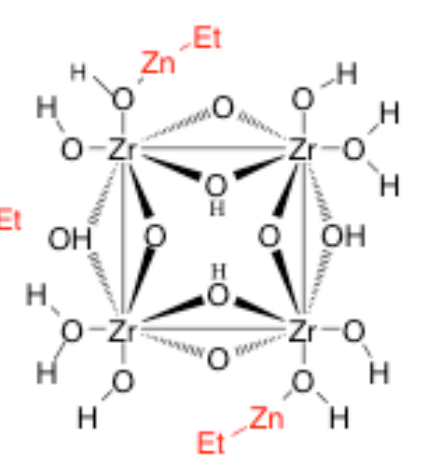

$-63.7$

Scheme S1. The possible reaction pathways of a second equivalent of $\mathrm{ZnEt}_{2}$ adding to the NU-1000 node after reaction of one equivalent of $\mathrm{ZnEt}_{2}$ with the $\mathrm{HB}$ site. $\Delta G_{383}^{\circ}$ reported in $\mathrm{kcal} / \mathrm{mol}$. 

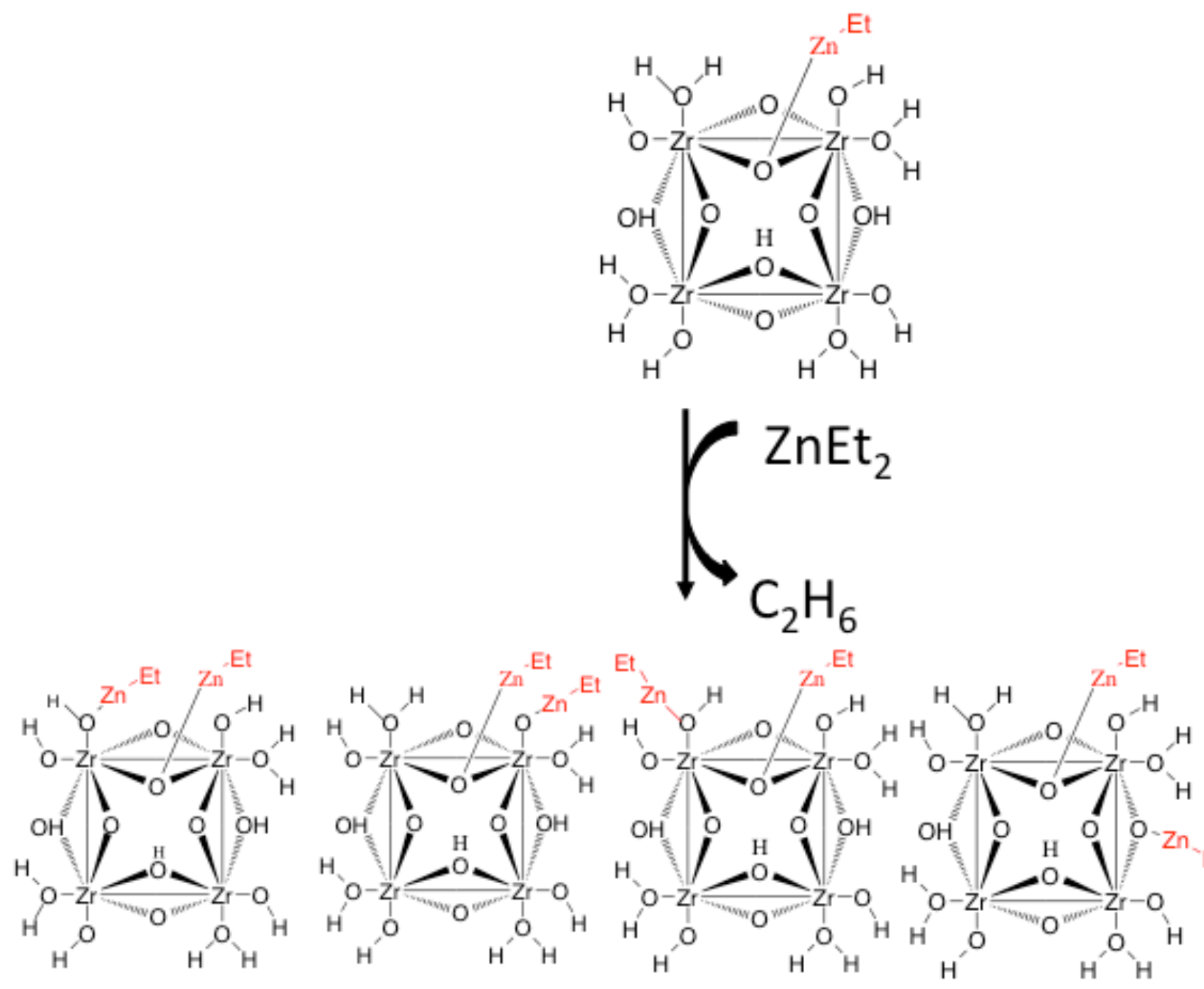

$-73.8$
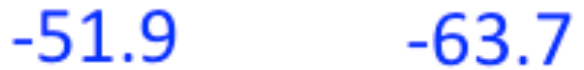

$-60.8$

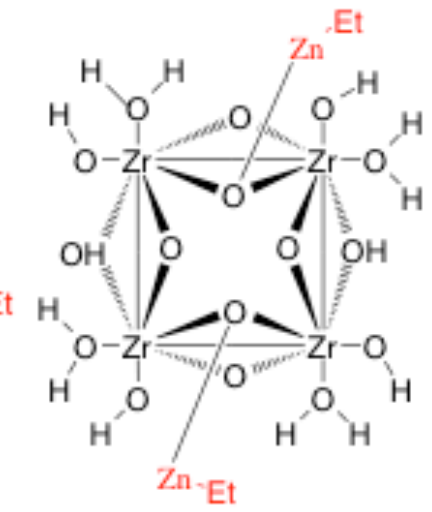

Scheme S2. The possible reaction pathways of a second equivalent of $\mathrm{ZnEt}_{2}$ adding to the NU-1000 node after reaction of one equivalent of $\mathrm{ZnEt}_{2}$ with the $\mu \mathrm{OH}$ site. $\Delta G_{383}^{\circ}$ reported in $\mathrm{kcal} / \mathrm{mol}$. 


\section{Quantum Mechanical Results for Zn Coordination}

Although optimizations were started with 4-, 5-, and 6-coordinated Zn structures, after the optimization was complete and energies and bond distances were analyzed, all the structures were found to be 4-coordinate. Thus, Figure S10 shows the resulting fourcoordinate $\mathrm{Zn}$ species whose geometries were quantum mechanically optimized for this study. The more water molecules that are added to the system, the lower the $\Delta G_{383}^{\circ}$. As the $\mathrm{Zn}$ ion coordinates to more water molecules, the distance between the $\mathrm{Zr}-\mu_{3}-\mathrm{O} / \mathrm{Zr}-$ $\mu_{3}-\mathrm{OH}$ and the $\mathrm{Zn}$ ion increases.

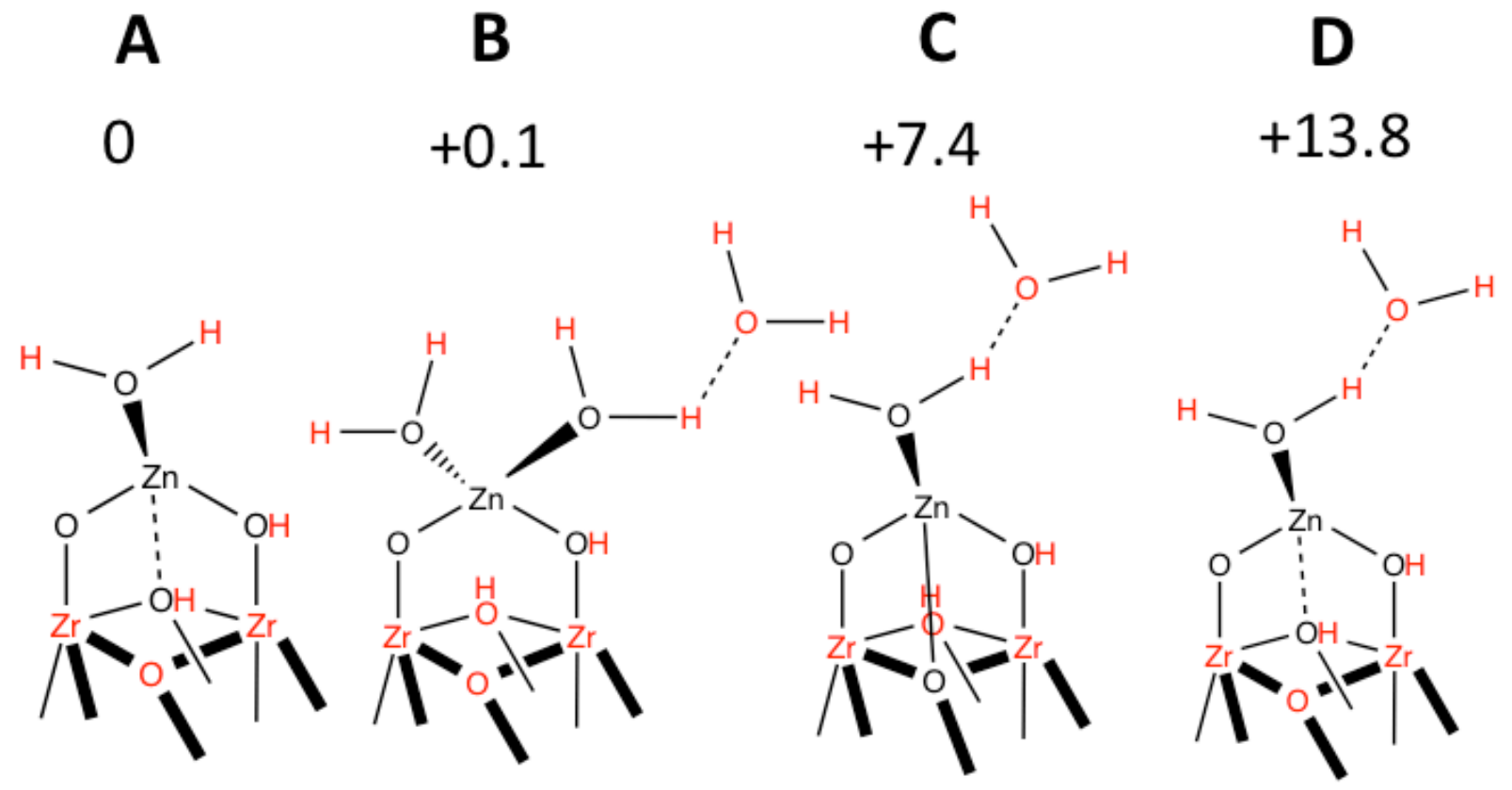

Figure S10. The four different four-coordinated species are shown in order of smallest to largest $\Delta G_{383}^{\circ}(\mathrm{kcal} / \mathrm{mol})$. 


\section{Coordinates for Computational Structures}

The coordinates for the bare node can be found in reference 1 .

Table S3. Structure for $\mathrm{ZnEt}_{2}$.

$E=-385.54644376$ Hartrees, $G=-385.464842$ Hartrees

\begin{tabular}{lrrr}
\hline Atom & \multicolumn{1}{c}{$\mathrm{X}$} & $\mathrm{Y}$ & \multicolumn{1}{c}{$\mathrm{Z}$} \\
\hline \hline $\mathrm{Zn}$ & 0.000562 & -0.000121 & -0.000046 \\
$\mathrm{C}$ & -0.029962 & 1.949937 & -0.051979 \\
$\mathrm{H}$ & -0.502905 & 2.308343 & 0.874294 \\
$\mathrm{H}$ & 1.008714 & 2.308647 & -0.0011 \\
$\mathrm{C}$ & 0.02961 & -1.950229 & 0.050825 \\
$\mathrm{H}$ & 0.504702 & -2.308438 & -0.874423 \\
$\mathrm{H}$ & -1.009154 & -2.308275 & -0.002915 \\
$\mathrm{C}$ & -0.729947 & 2.566183 & -1.260517 \\
$\mathrm{H}$ & -1.784116 & 2.267394 & -1.324135 \\
$\mathrm{H}$ & -0.71537 & 3.665583 & -1.235004 \\
$\mathrm{H}$ & -0.260353 & 2.267667 & -2.206542 \\
$\mathrm{C}$ & 0.726128 & -2.567538 & 1.260806 \\
$\mathrm{H}$ & 0.254295 & -2.269278 & 2.205799 \\
$\mathrm{H}$ & 0.710956 & -3.666912 & 1.234617 \\
$\mathrm{H}$ & 1.780309 & -2.269411 & 1.327281
\end{tabular}

Table S4. Structure for ethane.

$E=-79.807435249$ Hartrees, $G=-79.763306$ Hartrees

\begin{tabular}{lrrr}
\hline Atom & \multicolumn{1}{c}{$\mathrm{X}$} & $\mathrm{Y}$ & $\mathrm{Z}$ \\
\hline \hline $\mathrm{C}$ & 0.000037 & 0.000000 & 0.005236 \\
$\mathrm{C}$ & -0.000037 & 0.000000 & 1.522232 \\
$\mathrm{H}$ & 1.019516 & 0.000000 & -0.397933 \\
$\mathrm{H}$ & -0.509738 & -0.883036 & -0.397861 \\
$\mathrm{H}$ & -0.509738 & 0.883036 & -0.397861 \\
$\mathrm{H}$ & -1.019429 & 0.000000 & 1.925448 \\
$\mathrm{H}$ & 0.509694 & -0.882961 & 1.925369 \\
$\mathrm{H}$ & 0.509694 & 0.882961 & 1.925369
\end{tabular}

Table S5. Structure for water.

$E=-76.4000298357$ Hartrees, $G=-76.402337$ Hartrees

\begin{tabular}{lccc}
\hline Atom & $\mathrm{X}$ & $\mathrm{Y}$ & $\mathrm{Z}$ \\
\hline $\mathrm{O}$ & 0.000000 & 0.000000 & 0.119427 \\
$\mathrm{H}$ & 0.000000 & 0.757116 & -0.477707 \\
$\mathrm{H}$ & 0.000000 & -0.757116 & -0.477707
\end{tabular}


Table S6. $\mathrm{H}_{2} \mathrm{O}-1 \mathrm{Et}$ structure.

$E=-5164.83355773$ Hartrees, $G=-5164.12771$ Hatrees

\begin{tabular}{|c|c|c|c|}
\hline Atom & $X$ & $\mathrm{Y}$ & Z \\
\hline C & 6.080791 & 2.435556 & 2.473975 \\
\hline $\mathrm{C}$ & -5.248547 & 3.548316 & -2.567039 \\
\hline C & 6.04886 & 2.276425 & -2.661696 \\
\hline $\mathrm{C}$ & -5.182261 & 3.746579 & 2.39208 \\
\hline C & 4.778669 & 2.837576 & 2.806422 \\
\hline C & -3.88354 & 3.674775 & -2.865282 \\
\hline C & 4.744345 & 2.676728 & -2.987874 \\
\hline $\mathrm{C}$ & -3.820499 & 3.885316 & 2.699138 \\
\hline C & 4.602891 & 3.963099 & 3.625462 \\
\hline C & -3.477365 & 4.633086 & -3.805749 \\
\hline C & 4.565589 & 3.710736 & -3.918979 \\
\hline C & -3.419591 & 4.913316 & 3.564873 \\
\hline C & 5.704726 & 4.67913 & 4.087597 \\
\hline C & -4.413127 & 5.467062 & -4.410171 \\
\hline C & 5.664503 & 4.351661 & -4.484465 \\
\hline C & -4.356173 & 5.800722 & 4.087765 \\
\hline C & 7.015926 & 4.285788 & 3.755148 \\
\hline C & -5.782075 & 5.371815 & -4.092407 \\
\hline C & 6.978471 & 3.975413 & -4.142788 \\
\hline C & -5.72296 & 5.6843 & 3.766238 \\
\hline C & 7.180313 & 3.145755 & 2.945087 \\
\hline C & -6.181838 & 4.381873 & -3.175209 \\
\hline C & 7.145931 & 2.912742 & -3.234108 \\
\hline C & -6.117924 & 4.628949 & 2.92247 \\
\hline C & -5.818904 & -2.074146 & -2.353199 \\
\hline C & 5.486577 & -3.050228 & 2.67746 \\
\hline C & -5.826659 & -1.872284 & 2.615256 \\
\hline C & 5.470797 & -3.209265 & -2.482416 \\
\hline C & -4.520631 & -2.504124 & -2.665248 \\
\hline C & 4.128055 & -3.167621 & 3.008584 \\
\hline C & -4.519913 & -2.291982 & 2.905547 \\
\hline C & 4.111098 & -3.3275 & -2.809894 \\
\hline C & -4.352559 & -3.593571 & -3.532035 \\
\hline C & 3.740089 & -4.138247 & 3.944939 \\
\hline C & -4.324758 & -3.314842 & 3.845397 \\
\hline C & 3.701078 & -4.388104 & -3.632121 \\
\hline C & -5.459378 & -4.258671 & -4.050655 \\
\hline C & 4.683199 & -4.995098 & 4.505878 \\
\hline C & -5.4152 & -3.925956 & 4.456863 \\
\hline
\end{tabular}




\begin{tabular}{|c|c|c|c|}
\hline$C$ & 4.626345 & -5.319129 & -4.098477 \\
\hline$C$ & -6.767285 & -3.85167 & -3.722691 \\
\hline$C$ & 6.045494 & -4.905486 & 4.157442 \\
\hline$C$ & -6.732664 & -3.536195 & 4.146572 \\
\hline$C$ & 5.99177 & -5.21438 & -3.76788 \\
\hline$C$ & -6.92394 & -2.735511 & -2.879383 \\
\hline$C$ & 6.428983 & -3.90132 & 3.247108 \\
\hline$C$ & -6.914918 & -2.484123 & 3.229653 \\
\hline$C$ & 6.394936 & -4.134912 & -2.958268 \\
\hline$C$ & 3.584085 & 2.019751 & -2.317546 \\
\hline C & 3.608108 & 2.114655 & 2.235946 \\
\hline$C$ & 3.129045 & -2.283752 & 2.336666 \\
\hline$C$ & -3.353878 & -1.677441 & 2.215009 \\
\hline$C$ & -3.347459 & -1.803604 & -2.067639 \\
\hline C & -2.874046 & 2.821225 & -2.18119 \\
\hline$C$ & 3.119668 & -2.378742 & -2.227753 \\
\hline$C$ & -2.828026 & 2.945382 & 2.100673 \\
\hline $\mathrm{H}$ & 6.210911 & 1.566486 & 1.833387 \\
\hline $\mathrm{H}$ & -5.557972 & 2.795346 & -1.845833 \\
\hline $\mathrm{H}$ & 6.18382 & 1.469603 & -1.945521 \\
\hline $\mathrm{H}$ & -5.487134 & 2.940514 & 1.728871 \\
\hline $\mathrm{H}$ & 3.591892 & 4.279919 & 3.871579 \\
\hline $\mathrm{H}$ & -2.420921 & 4.714024 & -4.050558 \\
\hline $\mathrm{H}$ & 3.553541 & 4.022351 & -4.168916 \\
\hline $\mathrm{H}$ & -2.366319 & 5.004567 & 3.821524 \\
\hline $\mathrm{H}$ & 5.547542 & 5.557204 & 4.71221 \\
\hline $\mathrm{H}$ & -4.079012 & 6.21064 & -5.132357 \\
\hline $\mathrm{H}$ & 5.502933 & 5.163346 & -5.19223 \\
\hline $\mathrm{H}$ & -4.025282 & 6.596691 & 4.753251 \\
\hline $\mathrm{H}$ & 8.183392 & 2.818634 & 2.674843 \\
\hline $\mathrm{H}$ & -7.236401 & 4.274396 & -2.925339 \\
\hline $\mathrm{H}$ & 8.150741 & 2.591206 & -2.963263 \\
\hline $\mathrm{H}$ & -7.170226 & 4.508212 & 2.669014 \\
\hline $\mathrm{H}$ & -5.941759 & -1.224817 & -1.685107 \\
\hline $\mathrm{H}$ & 5.78423 & -2.290717 & 1.958651 \\
\hline $\mathrm{H}$ & -5.969985 & -1.073977 & 1.890504 \\
\hline $\mathrm{H}$ & 5.783147 & -2.388597 & -1.840892 \\
\hline $\mathrm{H}$ & -3.341407 & -3.91659 & -3.773051 \\
\hline $\mathrm{H}$ & 2.68715 & -4.22814 & 4.203675 \\
\hline $\mathrm{H}$ & -3.309371 & -3.628216 & 4.077121 \\
\hline $\mathrm{H}$ & 2.646394 & -4.481724 & -3.882876 \\
\hline $\mathrm{H}$ & -5.309854 & -5.111722 & -4.710892 \\
\hline $\mathrm{H}$ & 4.358443 & -5.753106 & 5.217184 \\
\hline
\end{tabular}




\begin{tabular}{|c|c|c|c|}
\hline $\mathrm{H}$ & -5.246453 & -4.725993 & 5.1763 \\
\hline $\mathrm{H}$ & 4.285683 & -6.141406 & -4.725938 \\
\hline $\mathrm{H}$ & -7.925297 & -2.393368 & -2.621125 \\
\hline $\mathrm{H}$ & 7.477618 & -3.797341 & 2.971859 \\
\hline $\mathrm{H}$ & -7.922884 & -2.152701 & 2.98423 \\
\hline $\mathrm{H}$ & 7.445272 & -4.026072 & -2.691476 \\
\hline $\mathrm{H}$ & 7.83685 & 4.48074 & -4.578667 \\
\hline $\mathrm{H}$ & -6.511222 & 6.030192 & -4.558386 \\
\hline $\mathrm{H}$ & 6.779752 & -5.580074 & 4.590816 \\
\hline $\mathrm{H}$ & 6.712695 & -5.943296 & -4.129914 \\
\hline $\mathrm{H}$ & -7.584176 & -4.022084 & 4.616818 \\
\hline $\mathrm{H}$ & -6.453497 & 6.379935 & 4.172082 \\
\hline $\mathrm{H}$ & -7.632091 & -4.375712 & -4.122764 \\
\hline $\mathrm{H}$ & 7.875819 & 4.846206 & 4.114012 \\
\hline $\mathrm{H}$ & -1.852509 & 0.37289 & -2.827418 \\
\hline $\mathrm{H}$ & 2.379679 & 2.857237 & -0.046934 \\
\hline $\mathrm{H}$ & 0.940075 & -1.343794 & 4.292876 \\
\hline $\mathrm{H}$ & 0.135922 & 0.011428 & 4.081898 \\
\hline $\mathrm{H}$ & 0.636277 & -1.651156 & -4.400491 \\
\hline $\mathrm{H}$ & 0.502078 & -4.16027 & -1.206319 \\
\hline $\mathrm{H}$ & -0.788017 & -4.096235 & 2.172313 \\
\hline $\mathrm{H}$ & -1.823963 & 0.454585 & 2.854197 \\
\hline $\mathrm{H}$ & 1.759727 & -2.833709 & 0.141365 \\
\hline $\mathrm{H}$ & 1.079502 & 1.924078 & 4.337468 \\
\hline $\mathrm{H}$ & 0.179161 & 0.446228 & -4.078127 \\
\hline $\mathrm{H}$ & 1.230633 & 1.623198 & -4.279112 \\
\hline $\mathrm{H}$ & -0.337218 & 4.597972 & 1.488359 \\
\hline $\mathrm{H}$ & -0.164298 & 4.584975 & -1.788273 \\
\hline $\mathrm{H}$ & 0.547971 & 4.264854 & 0.208781 \\
\hline 0 & 1.187318 & 0.108944 & 1.494387 \\
\hline $\mathrm{O}$ & 3.566067 & -1.537405 & 1.407983 \\
\hline 0 & 3.563114 & -1.536767 & -1.387042 \\
\hline 0 & 3.858129 & 1.186424 & 1.407437 \\
\hline 0 & 2.447821 & 2.494626 & 2.592713 \\
\hline $\mathrm{O}$ & 0.520855 & 4.220793 & 1.230314 \\
\hline 0 & -1.597511 & 3.146611 & 2.347833 \\
\hline 0 & -1.650811 & 3.003858 & -2.470604 \\
\hline $\mathrm{O}$ & -3.290909 & 2.005818 & 1.382613 \\
\hline 0 & -3.317463 & 1.968845 & -1.349515 \\
\hline $\mathrm{O}$ & -3.60808 & -0.751321 & 1.384354 \\
\hline $\mathrm{O}$ & -3.605062 & -0.800916 & -1.325068 \\
\hline $\mathrm{O}$ & -2.193573 & -2.238242 & -2.34662 \\
\hline $\mathrm{O}$ & -2.193613 & -2.107655 & 2.510456 \\
\hline
\end{tabular}




$\begin{array}{lrrr}\mathrm{O} & -1.129364 & -1.233099 & 0.010735 \\ \mathrm{O} & -1.241002 & 0.377509 & 2.084909 \\ \mathrm{O} & 0.266339 & 1.621602 & 3.907226 \\ \mathrm{O} & 0.068521 & -1.007333 & 4.024181 \\ \mathrm{O} & 0.31233 & 1.458202 & -4.005884 \\ \mathrm{O} & -0.764856 & 1.781101 & 0.015061 \\ \mathrm{O} & 1.736993 & 2.134478 & -0.00091 \\ \mathrm{O} & 2.415582 & 2.341496 & -2.692879 \\ \mathrm{O} & 0.562528 & 4.07693 & -1.399387 \\ \mathrm{O} & 3.859701 & 1.192604 & -1.391582 \\ \mathrm{O} & -0.281769 & -3.686934 & 1.452802 \\ \mathrm{O} & -0.303972 & -3.720362 & -1.510919 \\ \mathrm{O} & 1.285764 & -1.997846 & 0.029594 \\ \mathrm{O} & 1.916347 & -2.35722 & 2.714848 \\ \mathrm{O} & 1.912321 & -2.501126 & -2.593462 \\ \mathrm{O} & -0.033191 & -1.142757 & -3.921362 \\ \mathrm{O} & -1.258018 & 0.345125 & -2.063513 \\ \mathrm{O} & 1.177235 & 0.090528 & -1.45775 \\ \mathrm{Zr} & -2.364892 & 0.472355 & 0.008436 \\ \mathrm{Zr} & 0.348448 & 1.990082 & 1.833689 \\ \mathrm{Zr} & -0.076162 & -1.559654 & 1.778659 \\ \mathrm{Zr} & -0.041512 & -1.583914 & -1.866506 \\ \mathrm{Zr} & 2.622903 & -0.053415 & -0.002587 \\ \mathrm{Zr} & 0.295928 & 2.013836 & -1.763116 \\ \mathrm{C} & -3.550557 & -4.314674 & 0.261554 \\ \mathrm{H} & -4.181182 & -3.430709 & 0.072307 \\ \mathrm{H} & -3.636702 & -4.497897 & 1.343057 \\ \mathrm{Zn} & -1.702465 & -3.786933 & -0.112991 \\ \mathrm{C} & -4.092369 & -5.512172 & -0.509229 \\ \mathrm{H} & -3.562979 & -6.438641 & -0.249309 \\ \mathrm{H} & -5.161095 & -5.68686 & -0.308436 \\ \mathrm{H} & -3.994151 & -5.38628 & -1.594387\end{array}$


Table S7. Structure for HB-1Et.

$E=-5164.83313413$ Hartrees, $G=-5164.125865$ Hartrees

\begin{tabular}{|c|c|c|c|}
\hline Atom & $\mathrm{X}$ & $\mathrm{Y}$ & Z \\
\hline$C$ & -6.038168 & 2.359703 & -2.612139 \\
\hline$C$ & 5.251421 & 3.629597 & 2.480651 \\
\hline $\mathrm{C}$ & -6.053253 & 2.421137 & 2.525707 \\
\hline$C$ & 5.230784 & 3.615019 & -2.482808 \\
\hline $\mathrm{C}$ & -4.730905 & 2.740363 & -2.949517 \\
\hline$C$ & 3.884511 & 3.775774 & 2.760607 \\
\hline$C$ & -4.749523 & 2.828356 & 2.846416 \\
\hline C & 3.872625 & 3.74745 & -2.808066 \\
\hline $\mathrm{C}$ & -4.541545 & 3.82875 & -3.814436 \\
\hline C & 3.47522 & 4.775675 & 3.655313 \\
\hline$C$ & -4.573413 & 3.900469 & 3.733909 \\
\hline$C$ & 3.485211 & 4.739368 & -3.720765 \\
\hline$C$ & -5.635199 & 4.529936 & -4.316995 \\
\hline$C$ & 4.41012 & 5.630012 & 4.231995 \\
\hline$C$ & -5.673794 & 4.57074 & 4.26123 \\
\hline$C$ & 4.431354 & 5.598662 & -4.272596 \\
\hline$C$ & -6.951493 & 4.157992 & -3.98007 \\
\hline C & 5.78132 & 5.514156 & 3.931234 \\
\hline$C$ & -6.986704 & 4.186935 & 3.923919 \\
\hline$C$ & 5.794533 & 5.489122 & -3.933792 \\
\hline$C$ & -7.129462 & 3.054666 & -3.123394 \\
\hline$C$ & 6.183815 & 4.483689 & 3.061073 \\
\hline$C$ & -7.15188 & 3.087093 & 3.060163 \\
\hline$C$ & 6.176048 & 4.468963 & -3.041922 \\
\hline$C$ & 5.792518 & -1.999724 & 2.513485 \\
\hline$C$ & -5.472552 & -3.132746 & -2.574603 \\
\hline$C$ & 5.846022 & -2.011489 & -2.458773 \\
\hline$C$ & -5.504004 & -3.070095 & 2.58727 \\
\hline$C$ & 4.489132 & -2.409211 & 2.831721 \\
\hline$C$ & -4.111781 & -3.271246 & -2.887856 \\
\hline C & 4.539631 & -2.436532 & -2.742816 \\
\hline$C$ & -4.147977 & -3.181155 & 2.932032 \\
\hline$C$ & 4.307247 & -3.459545 & 3.742864 \\
\hline$C$ & -3.720803 & -4.283178 & -3.778093 \\
\hline $\mathrm{C}$ & 4.347263 & -3.497797 & -3.639676 \\
\hline C & -3.751242 & -4.207564 & 3.802746 \\
\hline $\mathrm{C}$ & 5.405662 & -4.107446 & 4.299715 \\
\hline $\mathrm{C}$ & -4.663568 & -5.158462 & -4.31042 \\
\hline$C$ & 5.439751 & -4.140217 & -4.214291 \\
\hline
\end{tabular}




\begin{tabular}{|c|c|c|c|}
\hline C & -4.685806 & -5.112922 & 4.300073 \\
\hline C & 6.718697 & -3.721648 & 3.966655 \\
\hline$C$ & -6.028421 & -5.046954 & -3.978715 \\
\hline$C$ & 6.756513 & -3.744278 & -3.908883 \\
\hline C & -6.047603 & -5.015439 & 3.95272 \\
\hline C & 6.8891 & -2.643561 & 3.077717 \\
\hline$C$ & -6.414503 & -4.002654 & -3.115872 \\
\hline$C$ & 6.936349 & -2.654747 & -3.036302 \\
\hline$C$ & -6.437495 & -3.96968 & 3.093863 \\
\hline C & -3.586946 & 2.13723 & 2.215606 \\
\hline$C$ & -3.569539 & 2.036594 & -2.337792 \\
\hline$C$ & -3.113968 & -2.36449 & -2.245301 \\
\hline$C$ & 3.370864 & -1.786905 & -2.090052 \\
\hline C & 3.325276 & -1.728973 & 2.193826 \\
\hline$C$ & 2.876489 & 2.89884 & 2.104492 \\
\hline C & -3.146119 & -2.263394 & 2.318881 \\
\hline$C$ & 2.869625 & 2.83921 & -2.178997 \\
\hline $\mathrm{H}$ & -6.178882 & 1.519546 & -1.936211 \\
\hline $\mathrm{H}$ & 5.562956 & 2.844709 & 1.795232 \\
\hline $\mathrm{H}$ & -6.186278 & 1.585208 & 1.843329 \\
\hline $\mathrm{H}$ & 5.525402 & 2.836764 & -1.782781 \\
\hline $\mathrm{H}$ & -3.52667 & 4.129431 & -4.06485 \\
\hline $\mathrm{H}$ & 2.417006 & 4.872495 & 3.886409 \\
\hline $\mathrm{H}$ & -3.561944 & 4.217229 & 3.979694 \\
\hline $\mathrm{H}$ & 2.434785 & 4.824893 & -3.990731 \\
\hline $\mathrm{H}$ & -5.467551 & 5.379491 & -4.977298 \\
\hline $\mathrm{H}$ & 4.073735 & 6.40572 & 4.918455 \\
\hline $\mathrm{H}$ & -5.514159 & 5.411226 & 4.934996 \\
\hline $\mathrm{H}$ & 4.110777 & 6.366953 & -4.974634 \\
\hline $\mathrm{H}$ & -8.136696 & 2.744462 & -2.848758 \\
\hline $\mathrm{H}$ & 7.23997 & 4.360246 & 2.825691 \\
\hline $\mathrm{H}$ & -8.155934 & 2.759302 & 2.794119 \\
\hline $\mathrm{H}$ & 7.225342 & 4.353819 & -2.77384 \\
\hline $\mathrm{H}$ & 5.925275 & -1.180916 & 1.810259 \\
\hline $\mathrm{H}$ & -5.772244 & -2.342492 & -1.890579 \\
\hline $\mathrm{H}$ & 5.987047 & -1.183523 & -1.767602 \\
\hline $\mathrm{H}$ & -5.806061 & -2.27753 & 1.906803 \\
\hline $\mathrm{H}$ & 3.29386 & -3.764176 & 3.996165 \\
\hline $\mathrm{H}$ & -2.665822 & -4.390774 & -4.021201 \\
\hline $\mathrm{H}$ & 3.332655 & -3.811759 & -3.872887 \\
\hline $\mathrm{H}$ & -2.698301 & -4.298456 & 4.060688 \\
\hline $\mathrm{H}$ & 5.24613 & -4.928484 & 4.997195 \\
\hline $\mathrm{H}$ & -4.336555 & -5.948653 & -4.984741 \\
\hline
\end{tabular}




\begin{tabular}{|c|c|c|c|}
\hline $\mathrm{H}$ & 5.272874 & -4.970129 & -4.89948 \\
\hline $\mathrm{H}$ & -4.355251 & -5.911002 & 4.963294 \\
\hline $\mathrm{H}$ & 7.894692 & -2.317806 & 2.814917 \\
\hline $\mathrm{H}$ & -7.464917 & -3.882002 & -2.854427 \\
\hline $\mathrm{H}$ & 7.943839 & -2.317748 & -2.796552 \\
\hline $\mathrm{H}$ & -7.484788 & -3.86723 & 2.81278 \\
\hline $\mathrm{H}$ & -7.846165 & 4.714886 & 4.329841 \\
\hline $\mathrm{H}$ & 6.509863 & 6.188266 & 4.375128 \\
\hline $\mathrm{H}$ & -6.762422 & -5.736103 & -4.389067 \\
\hline $\mathrm{H}$ & -6.775612 & -5.72506 & 4.338267 \\
\hline $\mathrm{H}$ & 7.609459 & -4.254372 & -4.350016 \\
\hline $\mathrm{H}$ & 6.532528 & 6.162905 & -4.362351 \\
\hline $\mathrm{H}$ & 7.576744 & -4.232104 & 4.397512 \\
\hline $\mathrm{H}$ & -7.805013 & 4.706813 & -4.370664 \\
\hline $\mathrm{H}$ & 1.8307 & 0.477163 & 2.836545 \\
\hline $\mathrm{H}$ & -2.356848 & 2.877456 & -0.082697 \\
\hline $\mathrm{H}$ & -0.906317 & -1.523286 & -4.226933 \\
\hline $\mathrm{H}$ & -0.099937 & -0.160725 & -4.068113 \\
\hline $\mathrm{H}$ & -0.747076 & -1.425377 & 4.420176 \\
\hline $\mathrm{H}$ & 0.816207 & -4.160723 & -2.053047 \\
\hline $\mathrm{H}$ & 0.605915 & -4.0469 & 2.417096 \\
\hline $\mathrm{H}$ & 1.851172 & 0.323542 & -2.83953 \\
\hline $\mathrm{H}$ & -1.619748 & -2.917092 & 0.095892 \\
\hline $\mathrm{H}$ & -1.030179 & 1.7417 & -4.402214 \\
\hline $\mathrm{H}$ & -0.190706 & 0.607983 & 4.057668 \\
\hline $\mathrm{H}$ & -1.248529 & 1.786061 & 4.212408 \\
\hline $\mathrm{H}$ & 0.386072 & 4.525008 & -1.667623 \\
\hline $\mathrm{H}$ & 0.178414 & 4.662958 & 1.607504 \\
\hline $\mathrm{H}$ & -0.515169 & 4.258818 & -0.383252 \\
\hline 0 & -1.170099 & 0.046748 & -1.477626 \\
\hline $\mathrm{O}$ & -3.555889 & -1.571595 & -1.359843 \\
\hline 0 & -3.57752 & -1.480137 & 1.412905 \\
\hline 0 & -3.833007 & 1.147194 & -1.471578 \\
\hline 0 & -2.404051 & 2.392501 & -2.702102 \\
\hline $\mathrm{O}$ & -0.478155 & 4.168017 & -1.401041 \\
\hline 0 & 1.642469 & 3.034329 & -2.446899 \\
\hline 0 & 1.651148 & 3.101827 & 2.371003 \\
\hline $\mathrm{O}$ & 3.320674 & 1.928884 & -1.416816 \\
\hline $\mathrm{O}$ & 3.323422 & 2.006931 & 1.316814 \\
\hline $\mathrm{O}$ & 3.623499 & -0.824463 & -1.301302 \\
\hline $\mathrm{O}$ & 3.59492 & -0.769023 & 1.402018 \\
\hline $\mathrm{O}$ & 2.166251 & -2.128663 & 2.504318 \\
\hline $\mathrm{O}$ & 2.211954 & -2.221275 & -2.382228 \\
\hline
\end{tabular}




$\begin{array}{lrrr}\mathrm{O} & 1.144538 & -1.249619 & 0.06344 \\ \mathrm{O} & 1.264056 & 0.281598 & -2.07061 \\ \mathrm{O} & -0.221739 & 1.452394 & -3.954231 \\ \mathrm{O} & -0.036362 & -1.176545 & -3.965544 \\ \mathrm{O} & -0.328929 & 1.614977 & 3.947641 \\ \mathrm{O} & 0.780872 & 1.775422 & -0.067331 \\ \mathrm{O} & -1.721369 & 2.14699 & -0.089582 \\ \mathrm{O} & -2.419653 & 2.469492 & 2.586236 \\ \mathrm{O} & -0.546295 & 4.141566 & 1.232613 \\ \mathrm{O} & -3.859808 & 1.269075 & 1.326656 \\ \mathrm{O} & 0.294411 & -3.75126 & -1.344798 \\ \mathrm{O} & 0.1964 & -3.65755 & 1.628652 \\ \mathrm{O} & -1.26138 & -2.015843 & 0.071067 \\ \mathrm{O} & -1.899528 & -2.456347 & -2.613579 \\ \mathrm{O} & -1.955167 & -2.32204 & 2.737956 \\ \mathrm{O} & 0.002832 & -1.002109 & 3.97737 \\ \mathrm{O} & 1.245084 & 0.429372 & 2.06688 \\ \mathrm{O} & -1.190654 & 0.166662 & 1.45045 \\ \mathrm{Zr} & 2.375253 & 0.459372 & 0.008971 \\ \mathrm{Zr} & -0.318538 & 1.910332 & -1.898804 \\ \mathrm{Zr} & 0.082531 & -1.630707 & -1.699688 \\ \mathrm{Zr} & 0.014838 & -1.489126 & 1.926833 \\ \mathrm{Zr} & -2.630946 & -0.050978 & 0.000557 \\ \mathrm{Zr} & -0.297344 & 2.095071 & 1.68578 \\ \mathrm{C} & 3.481263 & -4.399879 & 0.120284 \\ \mathrm{H} & 4.091265 & -3.516526 & -0.128852 \\ \mathrm{H} & 3.82962 & -4.710092 & 1.115881 \\ \mathrm{Zn} & 1.617328 & -3.799768 & 0.233966 \\ \mathrm{C} & 3.736437 & -5.51349 & -0.888173 \\ \mathrm{H} & 4.809119 & -5.72466 & -1.02356 \\ \mathrm{H} & 3.259441 & -6.454966 & -0.584025 \\ \mathrm{H} & 3.345129 & -5.270464 & -1.886004\end{array}$


Table S8. Structure for $\mathrm{OH}-1 \mathrm{Et}$.

$E=-5164.80656088$ Hartrees, $G=-5164.101887$ Hartrees

\begin{tabular}{|c|c|c|c|}
\hline Atom & $X$ & $\mathrm{Y}$ & Z \\
\hline C & 5.942684 & -1.790446 & -3.120797 \\
\hline C & -5.185229 & -4.203697 & 1.914055 \\
\hline C & 6.110628 & -2.914851 & 1.890089 \\
\hline $\mathrm{C}$ & -5.31209 & -3.160891 & -2.937031 \\
\hline C & 4.627586 & -2.106342 & -3.4928 \\
\hline C & -3.810006 & -4.390669 & 2.118964 \\
\hline C & 4.818718 & -3.393036 & 2.155988 \\
\hline $\mathrm{C}$ & -3.963647 & -3.209118 & -3.320834 \\
\hline C & 4.417185 & -2.993804 & -4.558947 \\
\hline C & -3.37013 & -5.549986 & 2.774945 \\
\hline C & 4.673545 & -4.627506 & 2.806441 \\
\hline C & -3.599356 & -3.986391 & -4.429953 \\
\hline C & 5.498362 & -3.564395 & -5.226625 \\
\hline C & -4.283897 & -6.514811 & 3.188024 \\
\hline C & 5.791905 & -5.381158 & 3.152057 \\
\hline C & -4.55786 & -4.722374 & -5.121104 \\
\hline C & 6.82251 & -3.256838 & -4.857126 \\
\hline C & -5.663897 & -6.353235 & 2.956603 \\
\hline C & 7.092596 & -4.922346 & 2.864808 \\
\hline C & -5.910821 & -4.699399 & -4.728661 \\
\hline C & 7.021217 & -2.353171 & -3.795619 \\
\hline C & -6.09638 & -5.169031 & 2.33077 \\
\hline C & 7.227427 & -3.665775 & 2.243624 \\
\hline C & -6.269953 & -3.890123 & -3.634219 \\
\hline C & -5.748719 & 1.290825 & 3.128892 \\
\hline C & 5.355399 & 3.568955 & -1.929058 \\
\hline C & -5.949938 & 2.331941 & -1.729291 \\
\hline C & 5.54042 & 2.43855 & 3.104609 \\
\hline C & -4.438185 & 1.638848 & 3.488264 \\
\hline C & 3.985354 & 3.755379 & -2.168292 \\
\hline C & -4.654354 & 2.819993 & -1.955716 \\
\hline C & 4.194779 & 2.461851 & 3.50299 \\
\hline C & -4.233725 & 2.479431 & 4.591931 \\
\hline C & 3.563865 & 4.925728 & -2.817925 \\
\hline C & -4.493162 & 4.04596 & -2.618037 \\
\hline C & 3.819779 & 3.281467 & 4.578473 \\
\hline $\mathrm{C}$ & -5.317822 & 2.986599 & 5.30178 \\
\hline C & 4.486724 & 5.901936 & -3.183507 \\
\hline C & -5.604922 & 4.782246 & -3.015898 \\
\hline
\end{tabular}




\begin{tabular}{|c|c|c|c|}
\hline$C$ & 4.764896 & 4.073688 & 5.226206 \\
\hline$C$ & -6.638542 & 2.664722 & 4.933096 \\
\hline$C$ & 5.861283 & 5.738137 & -2.920747 \\
\hline$C$ & -6.910359 & 4.318132 & -2.762253 \\
\hline$C$ & 6.116173 & 4.064263 & 4.827944 \\
\hline$C$ & -6.830751 & 1.792506 & 3.845063 \\
\hline$C$ & 6.277199 & 4.541749 & -2.304477 \\
\hline$C$ & -7.059625 & 3.069672 & -2.129889 \\
\hline$C$ & 6.484765 & 3.22319 & 3.760236 \\
\hline$C$ & 3.635029 & -2.598181 & 1.715255 \\
\hline C & 3.48196 & -1.556536 & -2.715931 \\
\hline$C$ & 3.01088 & 2.724982 & -1.699897 \\
\hline$C$ & -3.463999 & 2.06125 & -1.485083 \\
\hline$C$ & -3.290947 & 1.117509 & 2.690615 \\
\hline C & -2.825604 & -3.386531 & 1.630837 \\
\hline$C$ & 3.179008 & 1.680804 & 2.741323 \\
\hline$C$ & -2.946223 & -2.440672 & -2.545607 \\
\hline $\mathrm{H}$ & 6.100126 & -1.107274 & -2.289574 \\
\hline $\mathrm{H}$ & -5.519832 & -3.297485 & 1.414229 \\
\hline $\mathrm{H}$ & 6.219543 & -1.954342 & 1.39239 \\
\hline $\mathrm{H}$ & -5.588765 & -2.54857 & -2.082057 \\
\hline $\mathrm{H}$ & 3.396403 & -3.246517 & -4.836979 \\
\hline $\mathrm{H}$ & -2.305123 & -5.681947 & 2.950452 \\
\hline $\mathrm{H}$ & 3.671011 & -4.998503 & 3.009075 \\
\hline $\mathrm{H}$ & -2.556986 & -4.003239 & -4.741067 \\
\hline $\mathrm{H}$ & 5.314724 & -4.26064 & -6.043599 \\
\hline $\mathrm{H}$ & -3.924022 & -7.412595 & 3.688664 \\
\hline $\mathrm{H}$ & 5.655869 & -6.344749 & 3.640883 \\
\hline $\mathrm{H}$ & -4.255172 & -5.325573 & -5.975816 \\
\hline $\mathrm{H}$ & 8.034884 & -2.096373 & -3.491031 \\
\hline $\mathrm{H}$ & -7.159636 & -5.010755 & 2.155666 \\
\hline $\mathrm{H}$ & 8.221804 & -3.279893 & 2.022835 \\
\hline $\mathrm{H}$ & -7.311428 & -3.844007 & -3.318727 \\
\hline $\mathrm{H}$ & -5.898135 & 0.628608 & 2.279584 \\
\hline $\mathrm{H}$ & 5.678174 & 2.657072 & -1.432676 \\
\hline $\mathrm{H}$ & -6.066341 & 1.375085 & -1.225607 \\
\hline $\mathrm{H}$ & 5.825535 & 1.805706 & 2.267511 \\
\hline $\mathrm{H}$ & -3.214909 & 2.72658 & 4.882582 \\
\hline $\mathrm{H}$ & 2.501498 & 5.068853 & -3.005113 \\
\hline $\mathrm{H}$ & -3.489808 & 4.406089 & -2.830161 \\
\hline $\mathrm{H}$ & 2.774703 & 3.306755 & 4.879163 \\
\hline $\mathrm{H}$ & -5.140872 & 3.643263 & 6.152328 \\
\hline $\mathrm{H}$ & 4.136719 & 6.811105 & -3.670462 \\
\hline
\end{tabular}




\begin{tabular}{|c|c|c|c|}
\hline $\mathrm{H}$ & -5.459852 & 5.736762 & -3.520544 \\
\hline $\mathrm{H}$ & 4.450912 & 4.712777 & 6.050254 \\
\hline $\mathrm{H}$ & -7.842194 & 1.516243 & 3.550156 \\
\hline $\mathrm{H}$ & 7.335553 & 4.380392 & -2.103914 \\
\hline $\mathrm{H}$ & -8.058116 & 2.679207 & -1.938894 \\
\hline $\mathrm{H}$ & 7.523522 & 3.192222 & 3.434151 \\
\hline $\mathrm{H}$ & 7.965935 & -5.514177 & 3.127975 \\
\hline $\mathrm{H}$ & -6.376109 & -7.112137 & 3.271423 \\
\hline $\mathrm{H}$ & 6.579927 & 6.504634 & -3.200276 \\
\hline $\mathrm{H}$ & 6.852547 & 4.685653 & 5.331806 \\
\hline $\mathrm{H}$ & -7.777974 & 4.899073 & -3.065497 \\
\hline $\mathrm{H}$ & -6.658372 & -5.277422 & -5.26661 \\
\hline $\mathrm{H}$ & -7.485545 & 3.06464 & 5.485436 \\
\hline $\mathrm{H}$ & 7.666357 & -3.704189 & -5.376862 \\
\hline $\mathrm{H}$ & -1.755 & -1.189703 & 2.838129 \\
\hline $\mathrm{H}$ & 2.344662 & -2.858089 & -0.655747 \\
\hline $\mathrm{H}$ & 0.729342 & 2.325708 & -3.712667 \\
\hline $\mathrm{H}$ & -0.067661 & 0.959465 & -3.817628 \\
\hline $\mathrm{H}$ & 0.817515 & 0.423417 & 4.695191 \\
\hline $\mathrm{H}$ & -0.300884 & 3.539437 & 1.274012 \\
\hline $\mathrm{H}$ & -1.234586 & 3.323792 & 2.538546 \\
\hline $\mathrm{H}$ & -1.956172 & 0.135778 & -2.706503 \\
\hline $\mathrm{H}$ & 1.747503 & 2.676355 & 0.71332 \\
\hline $\mathrm{H}$ & 0.867791 & -0.832641 & -4.608748 \\
\hline $\mathrm{H}$ & 0.29933 & -1.535691 & 3.927397 \\
\hline $\mathrm{H}$ & 1.372817 & -2.69795 & 3.797112 \\
\hline $\mathrm{H}$ & -0.447848 & -4.142061 & -2.472291 \\
\hline $\mathrm{H}$ & -0.13497 & -4.99852 & 0.688856 \\
\hline $\mathrm{H}$ & 0.499653 & -4.158151 & -1.199271 \\
\hline $\mathrm{O}$ & 1.103803 & 0.189311 & -1.385878 \\
\hline $\mathrm{O}$ & 3.48586 & 1.778129 & -0.997223 \\
\hline $\mathrm{O}$ & 3.593143 & 1.065181 & 1.711584 \\
\hline 0 & 3.771261 & -0.865676 & -1.690662 \\
\hline $\mathrm{O}$ & 2.307946 & -1.834678 & -3.114458 \\
\hline $\mathrm{O}$ & 0.429975 & -3.853696 & -2.169106 \\
\hline $\mathrm{O}$ & -1.728961 & -2.553128 & -2.888721 \\
\hline $\mathrm{O}$ & -1.592957 & -3.633746 & 1.81671 \\
\hline $\mathrm{O}$ & -3.381578 & -1.718631 & -1.595414 \\
\hline $\mathrm{O}$ & -3.29805 & -2.357127 & 1.058234 \\
\hline $\mathrm{O}$ & -3.680014 & 0.951685 & -0.917614 \\
\hline 0 & -3.572503 & 0.317243 & 1.745627 \\
\hline $\mathrm{O}$ & -2.124044 & 1.50358 & 3.010676 \\
\hline $\mathrm{O}$ & -2.323668 & 2.596843 & -1.674399 \\
\hline
\end{tabular}




$\begin{array}{lrrr}\mathrm{O} & -1.113668 & 1.014314 & 0.441536 \\ \mathrm{O} & -1.355843 & 0.06145 & -1.950762 \\ \mathrm{O} & 0.078169 & -0.656456 & -4.076913 \\ \mathrm{O} & -0.135404 & 1.929545 & -3.510215 \\ \mathrm{O} & 0.439135 & -2.496322 & 3.615209 \\ \mathrm{O} & -0.801579 & -1.852418 & -0.321636 \\ \mathrm{O} & 1.704537 & -2.151571 & -0.487033 \\ \mathrm{O} & 2.482179 & -3.018194 & 2.041069 \\ \mathrm{O} & 0.581625 & -4.404354 & 0.422112 \\ \mathrm{O} & 3.877876 & -1.56632 & 1.015616 \\ \mathrm{O} & -0.30531 & 3.784664 & -0.355084 \\ \mathrm{O} & -0.309304 & 3.245134 & 2.248771 \\ \mathrm{O} & 1.285997 & 1.83913 & 0.561556 \\ \mathrm{O} & 1.791067 & 2.874475 & -2.009865 \\ \mathrm{O} & 1.980582 & 1.698749 & 3.164169 \\ \mathrm{O} & 0.07921 & 0.069714 & 4.178202 \\ \mathrm{O} & -1.200439 & -0.968316 & 2.076075 \\ \mathrm{O} & 1.214434 & -0.561487 & 1.467783 \\ \mathrm{Zr} & -2.377519 & -0.579846 & 0.066665 \\ \mathrm{Zr} & 0.247821 & -1.530826 & -2.167652 \\ \mathrm{Zr} & -0.169836 & 1.919343 & -1.182732 \\ \mathrm{Zr} & 0.018654 & 0.938909 & 2.261021 \\ \mathrm{Zr} & 2.598698 & -0.020755 & 0.019406 \\ \mathrm{Zr} & 0.332702 & -2.50339 & 1.296354 \\ \mathrm{C} & -2.737617 & 6.473675 & -1.179022 \\ \mathrm{H} & -2.788405 & 6.627083 & -2.267101 \\ \mathrm{H} & -3.756902 & 6.198042 & -0.875386 \\ \mathrm{Zn} & -1.59262 & 4.948756 & -0.851394 \\ \mathrm{C} & -2.301461 & 7.760489 & -0.487191 \\ \mathrm{H} & -1.298196 & 8.07849 & -0.796741 \\ \mathrm{H} & -2.982617 & 8.594399 & -0.710445 \\ \mathrm{H} & -2.277847 & 7.655209 & 0.604519\end{array}$


Table S9. Structure for $\mu \mathrm{OH}-1 \mathrm{Et}$.

$E=-5164.82567276$ Hartrees, $G=-5164.120319$ Hartrees

\begin{tabular}{lrrr}
\hline Atom & \multicolumn{1}{c}{$\mathrm{X}$} & \multicolumn{1}{c}{$\mathrm{Y}$} & \multicolumn{1}{c}{$\mathrm{Z}$} \\
\hline \hline $\mathrm{C}$ & 5.116073 & 3.62366 & 2.593909 \\
$\mathrm{C}$ & -6.187201 & 2.28712 & -2.451252 \\
$\mathrm{C}$ & 5.09328 & 3.70143 & -2.543687 \\
$\mathrm{C}$ & -6.141167 & 2.263637 & 2.512003 \\
$\mathrm{C}$ & 3.757918 & 3.693675 & 2.937676 \\
$\mathrm{C}$ & -4.891443 & 2.743428 & -2.735822 \\
$\mathrm{C}$ & 3.729482 & 3.799966 & -2.857911 \\
$\mathrm{C}$ & -4.848021 & 2.702849 & 2.832617 \\
$\mathrm{C}$ & 3.328147 & 4.707261 & 3.807427 \\
$\mathrm{C}$ & -4.726234 & 3.813034 & -3.628273 \\
$\mathrm{C}$ & 3.308357 & 4.805697 & -3.740657 \\
$\mathrm{C}$ & -4.694047 & 3.754566 & 3.747599 \\
$\mathrm{C}$ & 4.234314 & 5.638978 & 4.308499 \\
$\mathrm{C}$ & -5.834638 & 4.43203 & -4.198166 \\
$\mathrm{C}$ & 4.223463 & 5.711774 & -4.269594 \\
$\mathrm{C}$ & -5.80939 & 4.372623 & 4.306265 \\
$\mathrm{C}$ & 5.599263 & 5.579515 & 3.965184 \\
$\mathrm{C}$ & -7.141423 & 4.004195 & -3.892658 \\
$\mathrm{C}$ & 5.590908 & 5.638086 & -3.938715 \\
$\mathrm{C}$ & -7.112729 & 3.954581 & 3.97221 \\
$\mathrm{C}$ & 6.021407 & 4.548753 & 3.103661 \\
$\mathrm{C}$ & -7.293124 & 2.906461 & -3.024894 \\
$\mathrm{C}$ & 6.007636 & 4.602924 & -3.079768 \\
$\mathrm{C}$ & -7.254394 & 2.876667 & 3.077927 \\
$\mathrm{C}$ & -5.424157 & -3.316287 & -2.503391 \\
$\mathrm{C}$ & 5.823866 & -1.852122 & 2.53768 \\
$\mathrm{C}$ & -5.450896 & -3.354046 & 2.468954 \\
$\mathrm{C}$ & 5.816616 & -1.76935 & -2.624002 \\
$\mathrm{C}$ & -4.063082 & -3.415304 & -2.828109 \\
$\mathrm{C}$ & 4.532476 & -2.299644 & 2.855554 \\
$\mathrm{C}$ & -4.08059 & -3.469218 & 2.746435 \\
$\mathrm{C}$ & 4.520561 & -2.187216 & -2.964045 \\
$\mathrm{C}$ & -3.649518 & -4.393433 & -3.743912 \\
$\mathrm{C}$ & -3.646081 & -4.460692 & 3.638528 \\
$\mathrm{C}$ & & -3.274761 & -3.83714 \\
$\mathrm{C}$ & -5.27422 & -4.299055 \\
$\mathrm{C}$ & -4.014211 & 4.26885
\end{tabular}




\begin{tabular}{|c|c|c|c|}
\hline C & -4.559672 & -5.337984 & 4.214834 \\
\hline C & 5.480484 & -3.940463 & -4.34143 \\
\hline C & -5.937745 & -5.200514 & -3.959564 \\
\hline C & 6.809951 & -3.591976 & 3.932406 \\
\hline C & -5.933502 & -5.253456 & 3.915917 \\
\hline C & 6.78528 & -3.534497 & -3.998869 \\
\hline C & -6.346595 & -4.192679 & -3.065905 \\
\hline C & 6.942539 & -2.48456 & 3.072074 \\
\hline C & -6.362177 & -4.231666 & 3.048163 \\
\hline C & 6.929083 & -2.429652 & -3.137533 \\
\hline C & 2.759085 & 2.859044 & -2.225332 \\
\hline C & 2.785938 & 2.744235 & 2.327686 \\
\hline C & 3.350524 & -1.643855 & 2.220225 \\
\hline C & -3.094757 & -2.567211 & 2.09172 \\
\hline C & -3.083167 & -2.488272 & -2.192040 \\
\hline C & -3.706324 & 2.118967 & -2.086861 \\
\hline C & 3.337867 & -1.525188 & -2.34363 \\
\hline C & -3.666471 & 2.050396 & 2.196315 \\
\hline $\mathrm{H}$ & 5.441486 & 2.840476 & 1.913064 \\
\hline $\mathrm{H}$ & -6.30788 & 1.450579 & -1.766793 \\
\hline $\mathrm{H}$ & 5.416225 & 2.916191 & -1.864302 \\
\hline $\mathrm{H}$ & -6.252952 & 1.440998 & 1.809716 \\
\hline $\mathrm{H}$ & 2.272418 & 4.766575 & 4.062139 \\
\hline $\mathrm{H}$ & -3.719596 & 4.150546 & -3.863341 \\
\hline $\mathrm{H}$ & 2.25004 & 4.882776 & -3.980789 \\
\hline $\mathrm{H}$ & -3.689848 & 4.077431 & 4.013986 \\
\hline $\mathrm{H}$ & 3.879407 & 6.425832 & 4.972472 \\
\hline $\mathrm{H}$ & -5.688213 & 5.266187 & -4.882899 \\
\hline $\mathrm{H}$ & 3.872393 & 6.495407 & -4.939421 \\
\hline $\mathrm{H}$ & -5.670346 & 5.192024 & 5.009973 \\
\hline $\mathrm{H}$ & 7.071571 & 4.478962 & 2.823385 \\
\hline $\mathrm{H}$ & -8.291906 & 2.543885 & -2.785817 \\
\hline $\mathrm{H}$ & 7.061259 & 4.513733 & -2.818244 \\
\hline $\mathrm{H}$ & -8.250708 & 2.524898 & 2.813564 \\
\hline $\mathrm{H}$ & -5.737976 & -2.548422 & -1.800586 \\
\hline $\mathrm{H}$ & 5.931187 & -1.013385 & 1.853704 \\
\hline $\mathrm{H}$ & -5.781297 & -2.575108 & 1.785791 \\
\hline $\mathrm{H}$ & 5.931756 & -0.932357 & -1.939021 \\
\hline $\mathrm{H}$ & -2.597602 & -4.449114 & -4.016117 \\
\hline $\mathrm{H}$ & 3.38635 & -3.725142 & 3.989027 \\
\hline $\mathrm{H}$ & -2.584913 & -4.532375 & 3.864949 \\
\hline $\mathrm{H}$ & 3.360299 & -3.606055 & -4.088713 \\
\hline $\mathrm{H}$ & -4.233267 & -6.031633 & -5.004098 \\
\hline
\end{tabular}




\begin{tabular}{|c|c|c|c|}
\hline $\mathrm{H}$ & 5.374408 & -4.860321 & 4.941234 \\
\hline $\mathrm{H}$ & -4.204988 & -6.10696 & 4.899799 \\
\hline $\mathrm{H}$ & 5.338501 & -4.791453 & -5.006016 \\
\hline $\mathrm{H}$ & -7.399055 & -4.105472 & -2.79942 \\
\hline $\mathrm{H}$ & 7.936065 & -2.12702 & 2.805319 \\
\hline $\mathrm{H}$ & -7.421365 & -4.133419 & 2.814408 \\
\hline $\mathrm{H}$ & 7.926218 & -2.092783 & -2.857411 \\
\hline $\mathrm{H}$ & 6.304727 & 6.350154 & -4.345758 \\
\hline $\mathrm{H}$ & -8.007066 & 4.494728 & -4.331179 \\
\hline $\mathrm{H}$ & 7.684114 & -4.095995 & 4.33704 \\
\hline $\mathrm{H}$ & 7.654809 & -4.057531 & -4.389425 \\
\hline $\mathrm{H}$ & -6.645205 & -5.945623 & 4.359497 \\
\hline $\mathrm{H}$ & -7.983514 & 4.440109 & 4.406144 \\
\hline $\mathrm{H}$ & -6.658129 & -5.891867 & -4.390229 \\
\hline $\mathrm{H}$ & 6.306119 & 6.308381 & 4.354361 \\
\hline $\mathrm{H}$ & -2.113457 & 0.01499 & -2.858755 \\
\hline $\mathrm{H}$ & 1.422419 & 3.277867 & 0.087731 \\
\hline $\mathrm{H}$ & 1.014038 & -1.35405 & 4.163527 \\
\hline $\mathrm{H}$ & -0.084023 & -0.21843 & 4.025688 \\
\hline $\mathrm{H}$ & 0.816081 & -1.274392 & -4.386406 \\
\hline $\mathrm{H}$ & 0.111072 & -3.900414 & -0.516538 \\
\hline $\mathrm{H}$ & 0.173974 & -4.460819 & 1.68425 \\
\hline $\mathrm{H}$ & -0.584017 & -4.101251 & -1.922807 \\
\hline $\mathrm{H}$ & -2.099434 & -0.157958 & 2.845468 \\
\hline $\mathrm{H}$ & 0.383747 & 1.861205 & 4.407275 \\
\hline $\mathrm{H}$ & -0.186723 & 0.599461 & -4.04138 \\
\hline $\mathrm{H}$ & 0.568184 & 1.987978 & -4.204231 \\
\hline $\mathrm{H}$ & -1.643668 & 4.273612 & 1.681597 \\
\hline $\mathrm{H}$ & -1.49844 & 4.476912 & -1.581702 \\
\hline $\mathrm{H}$ & -0.702916 & 4.228365 & 0.402117 \\
\hline $\mathrm{O}$ & 0.893242 & 0.269803 & 1.492417 \\
\hline $\mathrm{O}$ & 3.596049 & -0.760951 & 1.345482 \\
\hline 0 & 3.577971 & -0.633587 & -1.472268 \\
\hline $\mathrm{O}$ & 3.236326 & 1.936381 & 1.458993 \\
\hline $\mathrm{O}$ & 1.570699 & 2.829867 & 2.69757 \\
\hline $\mathrm{O}$ & -0.719759 & 4.125771 & 1.417935 \\
\hline $\mathrm{O}$ & -2.517458 & 2.520305 & 2.462041 \\
\hline $\mathrm{O}$ & -2.564442 & 2.602875 & -2.358306 \\
\hline $\mathrm{O}$ & -3.909178 & 1.070089 & 1.42798 \\
\hline $\mathrm{O}$ & -3.939142 & 1.15138 & -1.299316 \\
\hline 0 & -3.573761 & -1.675807 & 1.321918 \\
\hline $\mathrm{O}$ & -3.566987 & -1.599284 & -1.423525 \\
\hline $\mathrm{O}$ & -1.856441 & -2.635769 & -2.475951 \\
\hline
\end{tabular}




$\begin{array}{lrrr}\mathrm{O} & -1.860599 & -2.749484 & 2.336346 \\ \mathrm{O} & -1.082769 & -1.44404 & -0.08319 \\ \mathrm{O} & -1.53374 & -0.0593 & 2.066175 \\ \mathrm{O} & -0.342385 & 1.405234 & 3.957235 \\ \mathrm{O} & 0.085547 & -1.216849 & 3.911593 \\ \mathrm{O} & -0.291643 & 1.611858 & -3.949918 \\ \mathrm{O} & -1.407102 & 1.521846 & 0.069763 \\ \mathrm{O} & 0.943525 & 2.436508 & 0.089533 \\ \mathrm{O} & 1.542604 & 2.931951 & -2.587387 \\ \mathrm{O} & -0.66569 & 4.13621 & -1.223903 \\ \mathrm{O} & 3.219972 & 2.07175 & -1.341304 \\ \mathrm{O} & 0.472363 & -3.739392 & 1.114748 \\ \mathrm{O} & 0.240597 & -3.786612 & -1.508719 \\ \mathrm{O} & 1.450811 & -1.693839 & -0.100502 \\ \mathrm{O} & 2.190885 & -2.046979 & 2.562465 \\ \mathrm{O} & 2.18467 & -1.916747 & -2.706872 \\ \mathrm{O} & -0.007468 & -1.022384 & -3.944151 \\ \mathrm{O} & -1.554315 & 0.10975 & -2.074139 \\ \mathrm{O} & 0.879449 & 0.425211 & -1.497314 \\ \mathrm{Zr} & -2.668366 & -0.121474 & -0.001484 \\ \mathrm{Zr} & -0.357308 & 1.8849 & 1.907065 \\ \mathrm{Zr} & 0.048815 & -1.659403 & 1.619203 \\ \mathrm{Zr} & 0.092217 & -1.451987 & -1.87712 \\ \mathrm{Zr} & 2.305291 & 0.485159 & -0.013173 \\ \mathrm{Zr} & -0.439643 & 2.091259 & -1.685652 \\ \mathrm{C} & 3.766623 & -4.722428 & 0.261761 \\ \mathrm{H} & 3.7368 & -5.024368 & 1.320439 \\ \mathrm{H} & 3.378516 & -5.582862 & -0.302687 \\ \mathrm{Zn} & 2.429941 & -3.320032 & 0.145571 \\ \mathrm{C} & 5.198771 & -4.419009 & -0.154576 \\ \mathrm{H} & 5.583407 & -3.503553 & 0.315043 \\ \mathrm{H} & 5.886718 & -5.232138 & 0.123856 \\ \mathrm{H} & 5.287923 & -4.280673 & -1.23876\end{array}$


Table S10. Structure for one $\mathrm{ZnEt}$ group bound to HB site and another to the $\mathrm{OH}$ site. $E=-5470.66821174$ Hartrees, $G=-5469.91829$ Hartrees

\begin{tabular}{|c|c|c|c|}
\hline Atom & $X$ & $\mathrm{Y}$ & Z \\
\hline$C$ & 5.652528 & 3.166724 & 2.651527 \\
\hline $\mathrm{C}$ & -5.722673 & 3.313047 & -2.406862 \\
\hline$C$ & 5.646154 & 3.357637 & -2.483152 \\
\hline$C$ & -5.686446 & 3.176976 & 2.554658 \\
\hline $\mathrm{C}$ & 4.312279 & 3.392669 & 2.998454 \\
\hline $\mathrm{C}$ & -4.380951 & 3.615698 & -2.683186 \\
\hline $\mathrm{C}$ & 4.304639 & 3.626748 & -2.793437 \\
\hline $\mathrm{C}$ & -4.350179 & 3.449925 & 2.883039 \\
\hline$C$ & 4.006784 & 4.431671 & 3.890452 \\
\hline$C$ & -4.0867 & 4.676587 & -3.552534 \\
\hline $\mathrm{C}$ & 4.009108 & 4.694777 & -3.653724 \\
\hline$C$ & -4.071664 & 4.455373 & 3.82032 \\
\hline$C$ & 5.018076 & 5.236206 & 4.410374 \\
\hline C & -5.111557 & 5.436968 & -4.107495 \\
\hline$C$ & 5.027558 & 5.495039 & -4.164126 \\
\hline$C$ & -5.105034 & 5.191276 & 4.39363 \\
\hline$C$ & 6.366338 & 5.019917 & 4.064112 \\
\hline$C$ & -6.460825 & 5.163434 & -3.809625 \\
\hline$C$ & 6.375678 & 5.249772 & -3.836661 \\
\hline$C$ & -6.448842 & 4.940858 & 4.05229 \\
\hline$C$ & 6.662183 & 3.964603 & 3.179983 \\
\hline C & -6.745024 & 4.07354 & -2.965584 \\
\hline$C$ & 6.663316 & 4.153585 & -3.00081 \\
\hline$C$ & -6.718333 & 3.907479 & 3.135113 \\
\hline$C$ & -5.641216 & -2.339019 & -2.581062 \\
\hline$C$ & 5.694547 & -2.351914 & 2.476151 \\
\hline$C$ & -5.678962 & -2.480659 & 2.389424 \\
\hline$C$ & 5.704239 & -2.157408 & -2.682597 \\
\hline$C$ & -4.301547 & -2.594573 & -2.909579 \\
\hline$C$ & 4.358163 & -2.647198 & 2.785842 \\
\hline$C$ & -4.332927 & -2.766348 & 2.662544 \\
\hline$C$ & 4.367642 & -2.408513 & -3.029875 \\
\hline$C$ & -4.007841 & -3.595434 & -3.846759 \\
\hline$C$ & 4.083434 & -3.717894 & 3.650426 \\
\hline$C$ & -4.022419 & -3.822051 & 3.532428 \\
\hline $\mathrm{C}$ & 4.083813 & -3.450255 & -3.926045 \\
\hline $\mathrm{C}$ & -5.029886 & -4.346169 & -4.419642 \\
\hline $\mathrm{C}$ & 5.118289 & -4.497079 & 4.160563 \\
\hline $\mathrm{C}$ & -5.035955 & -4.594924 & 4.09084 \\
\hline
\end{tabular}




$\begin{array}{lrrr}\mathrm{C} & 5.110901 & -4.234533 & -4.446056 \\ \mathrm{C} & -6.376444 & -4.115652 & -4.076904 \\ \mathrm{C} & 6.46164 & -4.227777 & 3.83167 \\ \mathrm{C} & -6.389149 & -4.338808 & 3.795589 \\ \mathrm{C} & 6.454688 & -3.996448 & -4.096488 \\ \mathrm{C} & -6.661902 & -3.08538 & -3.161167 \\ \mathrm{C} & 6.728025 & -3.126122 & 2.9953 \\ \mathrm{C} & -6.690249 & -3.254251 & 2.950668 \\ \mathrm{C} & 6.729595 & -2.935884 & -3.211674 \\ \mathrm{C} & 3.226971 & 2.79639 & -2.180148 \\ \mathrm{C} & 3.233694 & 2.580822 & 2.369258 \\ \mathrm{C} & 3.264801 & -1.840028 & 2.166075 \\ \mathrm{C} & -3.244525 & -1.976043 & 2.026133 \\ \mathrm{C} & -3.217855 & -1.80628 & -2.254626 \\ \mathrm{C} & -3.280724 & 2.838999 & -2.049475 \\ \mathrm{C} & 3.272704 & -1.621982 & -2.393351 \\ \mathrm{C} & -3.255231 & 2.673552 & 2.23126 \\ \mathrm{H} & 5.883361 & 2.364732 & 1.953969 \\ \mathrm{H} & -5.944272 & 2.481639 & -1.741228 \\ \mathrm{H} & 5.872536 & 2.52497 & -1.821235 \\ \mathrm{H} & -5.895824 & 2.388923 & 1.834806 \\ \mathrm{H} & 2.965607 & 4.613519 & 4.148195 \\ \mathrm{H} & -3.046203 & 4.895427 & -3.781571 \\ \mathrm{H} & 2.967996 & 4.90418 & -3.891451 \\ \mathrm{H} & -3.036265 & 4.649331 & 4.092995 \\ \mathrm{H} & 4.759701 & 6.045913 & 5.09144 \\ \mathrm{H} & -4.864445 & 6.262089 & -4.774286 \\ \mathrm{H} & 4.774352 & 6.329731 & -4.816384 \\ \mathrm{H} & -4.869032 & 5.972416 & 5.115047 \\ \mathrm{H} & 7.696777 & 3.774594 & 2.896972 \\ \mathrm{H} & -7.780676 & 3.828769 & -2.73327 \\ \mathrm{H} & 7.698275 & 3.93211 & -2.742793 \\ \mathrm{H} & -7.749561 & 3.684283 & 2.864359 \\ \mathrm{H} & -5.860926 & -1.558473 & -1.855942 \\ \mathrm{H} & 5.903691 & -1.517089 & 1.811019 \\ \mathrm{H} & -5.913292 & -1.656911 & 1.718282 \\ \mathrm{H} & 5.919231 & -1.35394 & -1.981532 \\ \mathrm{H} & -2.967474 & -3.780098 & -4.107318 \\ \mathrm{H} & 3.046613 & -3.949694 & 3.888582 \\ \mathrm{H} & -2.976395 & -4.033258 & 3.745367 \\ \mathrm{H} & 3.046457 & -3.651108 & -4.186796 \\ \mathrm{H} & -4.783093 & -5.127153 & -5.137751 \\ & 4.881451 & -5.336309 & 4.813199\end{array}$




$\begin{array}{rrrr}\mathrm{H} & -4.776186 & -5.420151 & 4.752641 \\ \mathrm{H} & 4.868299 & -5.047197 & -5.129521 \\ \mathrm{H} & -7.696564 & -2.87969 & -2.889224 \\ \mathrm{H} & 7.758095 & -2.884663 & 2.736099 \\ \mathrm{H} & -7.729583 & -3.024863 & 2.718805 \\ \mathrm{H} & 7.760085 & -2.726427 & -2.927405 \\ \mathrm{H} & 7.170768 & 5.879298 & -4.229185 \\ \mathrm{H} & -7.260477 & 5.764223 & -4.236544 \\ \mathrm{H} & 7.268171 & -4.842444 & 4.224044 \\ \mathrm{H} & 7.255312 & -4.611743 & -4.500026 \\ \mathrm{H} & -7.179883 & -4.950941 & 4.223174 \\ \mathrm{H} & -7.2554 & 5.518411 & 4.497931 \\ \mathrm{H} & -7.174473 & -4.70677 & -4.520245 \\ \mathrm{H} & 7.155456 & 5.649794 & 4.468085 \\ \mathrm{H} & -1.990063 & 0.536961 & -2.820601 \\ \mathrm{H} & 1.925959 & 3.337203 & 0.136803 \\ \mathrm{H} & 0.95934 & -1.303915 & 4.254226 \\ \mathrm{H} & 0.036926 & -0.019368 & 4.063346 \\ \mathrm{H} & 0.783697 & -1.040004 & -4.446848 \\ \mathrm{H} & -0.219195 & -3.975756 & -2.270682 \\ \mathrm{H} & -1.980935 & 0.286037 & 2.831786 \\ \mathrm{H} & 2.250891 & -2.119038 & -0.255685 \\ \mathrm{H} & 0.738746 & 1.974487 & 4.44407 \\ \mathrm{H} & 0.00951 & 0.926393 & -4.045852 \\ \mathrm{H} & 0.939978 & 2.204186 & -4.182634 \\ \mathrm{H} & -0.974279 & 4.625972 & 1.767444 \\ \mathrm{H} & -0.789116 & 4.882575 & -1.507875 \\ \mathrm{H} & -0.052561 & 4.496305 & 0.48136 \\ \mathrm{O} & 1.058051 & 0.359713 & 1.477102 \\ \mathrm{O} & 3.608598 & -0.981016 & 1.299083 \\ \mathrm{O} & 3.613704 & -0.803596 & -1.480714 \\ \mathrm{O} & 3.591748 & 1.749394 & 1.479697 \\ \mathrm{O} & 2.037513 & 2.7958 & 2.742187 \\ \mathrm{O} & -0.075346 & 4.376071 & 1.49399 \\ \mathrm{O} & -2.056958 & 2.994741 & 2.506706 \\ \mathrm{O} & -2.085252 & 3.17753 & -2.321489 \\ \mathrm{O} & -3.605047 & 1.738044 & 1.445794 \\ \mathrm{O} & -3.619347 & 1.888253 & -1.278139 \\ \mathrm{O} & -3.601429 & -1.044998 & 1.237586 \\ \mathrm{O} & -3.585763 & -0.891991 & -1.448955 \\ \mathrm{O} & -2.01818 & -2.090852 & -2.548999 \\ \mathrm{O} & -2.049508 & -2.273114 & 2.329451 \\ \mathrm{O} & -1.064701 & -1.142709 & -0.089504 \\ & & & \end{array}$




$\begin{array}{lrrr}\mathrm{O} & -1.383706 & 0.30837 & 2.069939 \\ \mathrm{O} & -0.028715 & 1.599873 & 3.987707 \\ \mathrm{O} & 0.07741 & -1.034912 & 3.949946 \\ \mathrm{O} & 0.040045 & 1.938603 & -3.928408 \\ \mathrm{O} & -1.06851 & 1.90584 & 0.094452 \\ \mathrm{O} & 1.372402 & 2.542912 & 0.124521 \\ \mathrm{O} & 2.029142 & 3.00561 & -2.54531 \\ \mathrm{O} & -0.012409 & 4.429539 & -1.14882 \\ \mathrm{O} & 3.595262 & 1.946795 & -1.308919 \\ \mathrm{O} & -0.016106 & -3.516394 & 1.235283 \\ \mathrm{O} & 0.059031 & -3.439396 & -1.512706 \\ \mathrm{O} & 1.45147 & -1.579847 & -0.147138 \\ \mathrm{O} & 2.070604 & -2.09741 & 2.526204 \\ \mathrm{O} & 2.08548 & -1.849057 & -2.778515 \\ \mathrm{O} & -0.006654 & -0.713703 & -3.992598 \\ \mathrm{O} & -1.387664 & 0.548477 & -2.062638 \\ \mathrm{O} & 1.054423 & 0.563506 & -1.490169 \\ \mathrm{Zr} & -2.494649 & 0.415911 & -0.005948 \\ \mathrm{Zr} & 0.010713 & 2.096061 & 1.939696 \\ \mathrm{Zr} & 0.015188 & -1.506741 & 1.694434 \\ \mathrm{Zr} & 0.045205 & -1.230582 & -1.951823 \\ \mathrm{Zr} & 2.490805 & 0.500631 & -0.012282 \\ \mathrm{Zr} & -0.047341 & 2.382013 & -1.656705 \\ \mathrm{C} & -3.280199 & -4.448355 & -0.310521 \\ \mathrm{H} & -4.057769 & -3.692226 & -0.118208 \\ \mathrm{H} & -3.386397 & -4.701572 & -1.374764 \\ \mathrm{Zn} & -1.537133 & -3.601316 & -0.031665 \\ \mathrm{C} & -3.53045 & -5.687321 & 0.542655 \\ \mathrm{H} & -4.561996 & -6.060321 & 0.441528 \\ \mathrm{H} & -2.863365 & -6.514994 & 0.265146 \\ \mathrm{H} & -3.369302 & -5.498296 & 1.611356 \\ \mathrm{Zn} & 1.386111 & -4.13818 & 0.154652 \\ \mathrm{C} & 3.173947 & -4.684522 & -0.397779 \\ \mathrm{H} & 3.80212 & -3.775034 & -0.371805 \\ \mathrm{H} & 3.111892 & -4.948193 & -1.464107 \\ \mathrm{H} & 3.871699 & -5.790055 & 0.384947 \\ \mathrm{H} & 3.993602 & -5.530262 & 1.443982 \\ \mathrm{H} & & -6.734357 & 0.349705\end{array}$


Table S11. Structure for one ZnEt group bound to HB site and another to the $\mu \mathrm{OH}$ site. $E=-5470.69056101$ Hartrees, $G=-5469.939505$ Hartrees

\begin{tabular}{lrrr}
\hline Atom & \multicolumn{1}{c}{$\mathrm{X}$} & $\mathrm{Y}$ & \multicolumn{1}{c}{$\mathrm{Z}$} \\
\hline \hline $\mathrm{C}$ & 5.621934 & 3.197609 & 2.631437 \\
$\mathrm{C}$ & -5.756286 & 3.219737 & -2.422233 \\
$\mathrm{C}$ & 5.612005 & 3.33449 & -2.504966 \\
$\mathrm{C}$ & -5.716926 & 3.136058 & 2.540429 \\
$\mathrm{C}$ & 4.280465 & 3.418803 & 2.976684 \\
$\mathrm{C}$ & -4.416604 & 3.527858 & -2.702409 \\
$\mathrm{C}$ & 4.268682 & 3.591964 & -2.817348 \\
$\mathrm{C}$ & -4.382245 & 3.42078 & 2.865214 \\
$\mathrm{C}$ & 3.96889 & 4.465205 & 3.857872 \\
$\mathrm{C}$ & -4.129391 & 4.581363 & -3.583008 \\
$\mathrm{C}$ & 3.966097 & 4.649024 & -3.688642 \\
$\mathrm{C}$ & -4.10958 & 4.437741 & 3.791742 \\
$\mathrm{C}$ & 4.975374 & 5.281457 & 4.368781 \\
$\mathrm{C}$ & -5.159234 & 5.329448 & -4.145398 \\
$\mathrm{C}$ & 4.979293 & 5.450213 & -4.207967 \\
$\mathrm{C}$ & -5.147263 & 5.173162 & 4.357833 \\
$\mathrm{C}$ & 6.324802 & 5.069962 & 4.024099 \\
$\mathrm{C}$ & -6.506629 & 5.050641 & -3.843965 \\
$\mathrm{C}$ & 6.32907 & 5.216819 & -3.878656 \\
$\mathrm{C}$ & -6.489638 & 4.910786 & 4.01985 \\
$\mathrm{C}$ & 6.626828 & 4.007282 & 3.150953 \\
$\mathrm{C}$ & -6.783625 & 3.967924 & -2.98837 \\
$\mathrm{C}$ & 6.623936 & 4.131293 & -3.031486 \\
$\mathrm{C}$ & -6.753089 & 3.866163 & 3.113723 \\
$\mathrm{C}$ & -5.639596 & -2.433256 & -2.53703 \\
$\mathrm{C}$ & 5.698348 & -2.322237 & 2.513997 \\
$\mathrm{C}$ & -5.674171 & -2.522888 & 2.434601 \\
$\mathrm{C}$ & 5.70445 & -2.181898 & -2.646441 \\
$\mathrm{C}$ & -4.298561 & -2.683805 & -2.863555 \\
$\mathrm{C}$ & 4.363986 & -2.622501 & 2.827568 \\
$\mathrm{C}$ & -4.32626 & -2.797225 & 2.710055 \\
$\mathrm{C}$ & 4.369335 & -2.444867 & -2.990416 \\
$\mathrm{C}$ & -3.998993 & -3.692618 & -3.790389 \\
$\mathrm{C}$ & -4.008756 & -3.841756 & 3.590846 \\
$\mathrm{C}$ & & -3.497771 & -3.875455 \\
$\mathrm{C}$ & -4.4567 & -4.453094 & 4.221207
\end{tabular}




\begin{tabular}{|c|c|c|c|}
\hline C & -5.017186 & -4.615051 & 4.157856 \\
\hline C & 5.123291 & -4.281072 & -4.387707 \\
\hline C & -6.364401 & -4.230035 & -4.013775 \\
\hline C & 6.47777 & -4.178896 & 3.888781 \\
\hline C & -6.372089 & -4.370514 & 3.860629 \\
\hline C & 6.465724 & -4.030953 & -4.041351 \\
\hline C & -6.655867 & -3.192005 & -3.108755 \\
\hline C & 6.736882 & -3.084455 & 3.040736 \\
\hline C & -6.680347 & -3.296805 & 3.004509 \\
\hline C & 6.734406 & -2.959457 & -3.16787 \\
\hline C & 3.196493 & 2.76134 & -2.194743 \\
\hline C & 3.20665 & 2.593688 & 2.356702 \\
\hline C & 3.265336 & -1.828721 & 2.200195 \\
\hline C & -3.243177 & -2.006778 & 2.064876 \\
\hline C & -3.219449 & -1.882147 & -2.217757 \\
\hline C & -3.311233 & 2.764686 & -2.061056 \\
\hline C & 3.269732 & -1.658743 & -2.362052 \\
\hline C & -3.282669 & 2.644473 & 2.221072 \\
\hline $\mathrm{H}$ & 5.856666 & 2.389438 & 1.942219 \\
\hline $\mathrm{H}$ & -5.972877 & 2.394064 & -1.747916 \\
\hline $\mathrm{H}$ & 5.843091 & 2.509879 & -1.834494 \\
\hline $\mathrm{H}$ & -5.922249 & 2.338867 & 1.829551 \\
\hline $\mathrm{H}$ & 2.926823 & 4.643016 & 4.114379 \\
\hline $\mathrm{H}$ & -3.090426 & 4.804188 & -3.815178 \\
\hline $\mathrm{H}$ & 2.923722 & 4.849782 & -3.927857 \\
\hline $\mathrm{H}$ & -3.075398 & 4.640859 & 4.062352 \\
\hline $\mathrm{H}$ & 4.71231 & 6.096635 & 5.041564 \\
\hline $\mathrm{H}$ & -4.91757 & 6.148919 & -4.821049 \\
\hline $\mathrm{H}$ & 4.720634 & 6.276409 & -4.868917 \\
\hline $\mathrm{H}$ & -4.915765 & 5.963146 & 5.070996 \\
\hline $\mathrm{H}$ & 7.662503 & 3.820876 & 2.869384 \\
\hline $\mathrm{H}$ & -7.817618 & 3.719221 & -2.75289 \\
\hline $\mathrm{H}$ & 7.660402 & 3.919238 & -2.771499 \\
\hline $\mathrm{H}$ & -7.783003 & 3.633699 & 2.845864 \\
\hline $\mathrm{H}$ & -5.864523 & -1.645636 & -1.821477 \\
\hline $\mathrm{H}$ & 5.901183 & -1.494176 & 1.838619 \\
\hline $\mathrm{H}$ & -5.913235 & -1.706781 & 1.756154 \\
\hline $\mathrm{H}$ & 5.914094 & -1.370353 & -1.953195 \\
\hline $\mathrm{H}$ & -2.957482 & -3.873538 & -4.048963 \\
\hline $\mathrm{H}$ & 3.062117 & -3.917231 & 3.951212 \\
\hline $\mathrm{H}$ & -2.962897 & -4.037382 & 3.816536 \\
\hline $\mathrm{H}$ & 3.055408 & -3.708544 & -4.133254 \\
\hline $\mathrm{H}$ & -4.765293 & -5.24249 & -5.064949 \\
\hline
\end{tabular}




\begin{tabular}{|c|c|c|c|}
\hline $\mathrm{H}$ & 4.905473 & -5.286809 & 4.883122 \\
\hline $\mathrm{H}$ & -4.75282 & -5.42958 & 4.831066 \\
\hline $\mathrm{H}$ & 4.885621 & -5.102861 & -5.062019 \\
\hline $\mathrm{H}$ & -7.691681 & -2.989644 & -2.838735 \\
\hline $\mathrm{H}$ & 7.765269 & -2.839676 & 2.777894 \\
\hline $\mathrm{H}$ & -7.72118 & -3.076109 & 2.771143 \\
\hline $\mathrm{H}$ & 7.76363 & -2.741246 & -2.88555 \\
\hline $\mathrm{H}$ & 7.120069 & 5.847109 & -4.278216 \\
\hline $\mathrm{H}$ & -7.310201 & 5.641867 & -4.27679 \\
\hline $\mathrm{H}$ & 7.288196 & -4.784462 & 4.287338 \\
\hline $\mathrm{H}$ & 7.269891 & -4.645856 & -4.438427 \\
\hline $\mathrm{H}$ & -7.158585 & -4.982581 & 4.295971 \\
\hline $\mathrm{H}$ & -7.29957 & 5.487877 & 4.459897 \\
\hline $\mathrm{H}$ & -7.15891 & -4.830638 & -4.45062 \\
\hline $\mathrm{H}$ & 7.110207 & 5.708908 & 4.421076 \\
\hline $\mathrm{H}$ & -1.989641 & 0.46732 & -2.819421 \\
\hline $\mathrm{H}$ & 1.916067 & 3.316307 & 0.119016 \\
\hline $\mathrm{H}$ & 0.996403 & -1.253855 & 4.192507 \\
\hline $\mathrm{H}$ & 0.029391 & -0.002517 & 4.051659 \\
\hline $\mathrm{H}$ & 0.799934 & -1.069913 & -4.423268 \\
\hline $\mathrm{H}$ & -0.071547 & -4.215548 & 1.852075 \\
\hline $\mathrm{H}$ & 0.00359 & -3.991287 & -2.316856 \\
\hline $\mathrm{H}$ & -1.966051 & 0.222856 & 2.827081 \\
\hline $\mathrm{H}$ & 0.720128 & 1.997178 & 4.424743 \\
\hline $\mathrm{H}$ & 0.000318 & 0.885151 & -4.039148 \\
\hline $\mathrm{H}$ & 0.911244 & 2.176245 & -4.180812 \\
\hline $\mathrm{H}$ & -1.020128 & 4.658277 & 1.727922 \\
\hline $\mathrm{H}$ & -0.848517 & 4.857239 & -1.535289 \\
\hline $\mathrm{H}$ & -0.091973 & 4.508607 & 0.445962 \\
\hline 0 & 1.06256 & 0.39995 & 1.524501 \\
\hline $\mathrm{O}$ & 3.606001 & -0.954458 & 1.35244 \\
\hline 0 & 3.606358 & -0.81217 & -1.478902 \\
\hline 0 & 3.561006 & 1.753888 & 1.476191 \\
\hline 0 & 2.008759 & 2.822824 & 2.727899 \\
\hline $\mathrm{O}$ & -0.122223 & 4.398679 & 1.461683 \\
\hline 0 & -2.08776 & 2.985755 & 2.484524 \\
\hline 0 & -2.120451 & 3.117079 & -2.326455 \\
\hline $\mathrm{O}$ & -3.628951 & 1.700838 & 1.445072 \\
\hline 0 & -3.649294 & 1.819642 & -1.281198 \\
\hline $\mathrm{O}$ & -3.617244 & -1.057103 & 1.304187 \\
\hline $\mathrm{O}$ & -3.601596 & -0.959009 & -1.426748 \\
\hline $\mathrm{O}$ & -2.016708 & -2.154116 & -2.512226 \\
\hline $\mathrm{O}$ & -2.035635 & -2.317744 & 2.31994 \\
\hline
\end{tabular}




\begin{tabular}{|c|c|c|c|}
\hline 0 & -1.16858 & -1.247317 & -0.088368 \\
\hline $\mathrm{O}$ & -1.38689 & 0.303179 & 2.055282 \\
\hline $\mathrm{O}$ & -0.058805 & 1.634595 & 3.977526 \\
\hline $\mathrm{O}$ & 0.093634 & -1.012472 & 3.926261 \\
\hline $\mathrm{O}$ & 0.013579 & 1.900578 & -3.92813 \\
\hline $\mathrm{O}$ & -1.084977 & 1.855307 & 0.09023 \\
\hline $\mathrm{O}$ & 1.339121 & 2.53893 & 0.116247 \\
\hline $\mathrm{O}$ & 1.996132 & 2.981466 & -2.554797 \\
\hline 0 & -0.067547 & 4.416286 & -1.170591 \\
\hline $\mathrm{O}$ & 3.562726 & 1.914933 & -1.32171 \\
\hline $\mathrm{O}$ & 0.093434 & -3.573406 & 1.14678 \\
\hline $\mathrm{O}$ & 0.12456 & -3.434447 & -1.534411 \\
\hline 0 & 1.351367 & -1.571154 & -0.095091 \\
\hline $\mathrm{O}$ & 2.066072 & -2.096576 & 2.549299 \\
\hline $\mathrm{O}$ & 2.082176 & -1.898209 & -2.742815 \\
\hline $\mathrm{O}$ & 0.00916 & -0.745924 & -3.968975 \\
\hline 0 & -1.406472 & 0.50984 & -2.047622 \\
\hline $\mathrm{O}$ & 1.049609 & 0.565898 & -1.494532 \\
\hline $\mathrm{Zr}$ & -2.533412 & 0.376899 & 0.000663 \\
\hline $\mathrm{Zr}$ & -0.01399 & 2.135143 & 1.933453 \\
\hline $\mathrm{Zr}$ & 0.046899 & -1.408168 & 1.631326 \\
\hline $\mathrm{Zr}$ & 0.061253 & -1.214177 & -1.90977 \\
\hline $\mathrm{Zr}$ & 2.476616 & 0.427758 & -0.01005 \\
\hline $\mathrm{Zr}$ & -0.067882 & 2.364507 & -1.662005 \\
\hline C & -3.176629 & -4.551559 & -0.154841 \\
\hline $\mathrm{H}$ & -3.978513 & -3.85611 & 0.13827 \\
\hline $\mathrm{H}$ & -3.452678 & -4.898365 & -1.160987 \\
\hline $\mathrm{Zn}$ & -1.499866 & -3.541759 & -0.251132 \\
\hline C & -3.125879 & -5.733278 & 0.807093 \\
\hline $\mathrm{H}$ & -4.109139 & -6.211268 & 0.941481 \\
\hline $\mathrm{H}$ & -2.435732 & -6.513925 & 0.457632 \\
\hline $\mathrm{H}$ & -2.78829 & -5.438236 & 1.810538 \\
\hline $\mathrm{Zn}$ & 1.846691 & -3.490307 & -0.21055 \\
\hline C & 3.351916 & -4.730616 & -0.080186 \\
\hline $\mathrm{H}$ & 3.587379 & -5.140986 & -1.072752 \\
\hline $\mathrm{H}$ & 4.240684 & -4.149979 & 0.212303 \\
\hline C & 3.117577 & -5.86224 & 0.915028 \\
\hline $\mathrm{H}$ & 2.288494 & -6.514871 & 0.606947 \\
\hline $\mathrm{H}$ & 4.001746 & -6.506943 & 1.041064 \\
\hline $\mathrm{H}$ & 2.86511 & -5.482209 & 1.915343 \\
\hline
\end{tabular}


Table S12. Structure for $\mathrm{H}_{2} \mathrm{O} / \mathrm{HB}-2 \mathrm{Et}$.

$E=-5470.66259018$ Hartrees, $G=-5469.913262$ Hartrees

\begin{tabular}{|c|c|c|c|}
\hline Atom & $X$ & $\mathrm{Y}$ & Z \\
\hline C & 6.217044 & 3.113757 & 1.710237 \\
\hline C & -5.144824 & 3.058682 & -3.37984 \\
\hline C & 6.105312 & 1.434739 & -3.144645 \\
\hline C & -5.000123 & 4.721335 & 1.294688 \\
\hline $\mathrm{C}$ & 4.932952 & 3.640312 & 1.913972 \\
\hline C & -3.781056 & 3.044395 & -3.708655 \\
\hline C & 4.809169 & 1.763742 & -3.569101 \\
\hline C & -3.630391 & 4.899 & 1.54004 \\
\hline C & 4.803996 & 4.963849 & 2.361353 \\
\hline C & -3.359228 & 3.665111 & -4.89381 \\
\hline C & 4.648883 & 2.479258 & -4.765106 \\
\hline C & -3.18533 & 6.123872 & 2.058326 \\
\hline C & 5.934048 & 5.747252 & 2.583992 \\
\hline C & -4.277493 & 4.312604 & -5.714806 \\
\hline C & 5.758709 & 2.885301 & -5.501031 \\
\hline C & -4.086323 & 7.157836 & 2.297535 \\
\hline C & 7.227483 & 5.228668 & 2.377626 \\
\hline C & -5.643951 & 4.362609 & -5.376635 \\
\hline C & 7.065269 & 2.58354 & -5.068991 \\
\hline C & -5.460599 & 6.997327 & 2.031744 \\
\hline C & 7.344737 & 3.894346 & 1.943192 \\
\hline C & -6.060687 & 3.704576 & -4.204301 \\
\hline C & 7.213053 & 1.834387 & -3.885801 \\
\hline C & -5.900243 & 5.752694 & 1.54255 \\
\hline C & -5.885435 & -2.22276 & -1.498087 \\
\hline C & 5.456663 & -2.039555 & 3.5415 \\
\hline C & -5.814484 & -0.551389 & 3.18463 \\
\hline C & 5.360722 & -3.726232 & -1.33659 \\
\hline C & -4.605755 & -2.769789 & -1.674006 \\
\hline C & 4.100136 & -2.007055 & 3.898972 \\
\hline C & -4.517234 & -0.909652 & 3.580594 \\
\hline C & 3.993387 & -3.890482 & -1.607554 \\
\hline C & -4.484081 & -4.072854 & -2.177675 \\
\hline C & 3.696066 & -2.641358 & 5.083783 \\
\hline C & -4.340087 & -1.612483 & 4.781549 \\
\hline C & 3.538847 & -5.133207 & -2.07455 \\
\hline C & -5.618367 & -4.824303 & -2.469383 \\
\hline C & 4.620333 & -3.323864 & 5.87005 \\
\hline $\mathrm{C}$ & -5.439854 & -1.976688 & 5.552447 \\
\hline
\end{tabular}




\begin{tabular}{|c|c|c|c|}
\hline$C$ & 4.42801 & -6.191582 & -2.246745 \\
\hline$C$ & -6.908153 & -4.294134 & -2.271383 \\
\hline$C$ & 5.97952 & -3.388149 & 5.504311 \\
\hline$C$ & -6.749032 & -1.652576 & 5.146358 \\
\hline$C$ & 5.800694 & -6.039463 & -1.968812 \\
\hline$C$ & -7.017931 & -2.972958 & -1.798138 \\
\hline$C$ & 6.380532 & -2.71395 & 4.334369 \\
\hline$C$ & -6.912049 & -0.915487 & 3.958527 \\
\hline$C$ & 6.248784 & -4.782264 & -1.519445 \\
\hline$C$ & 3.639066 & 1.375659 & -2.728022 \\
\hline$C$ & 3.732419 & 2.820422 & 1.590289 \\
\hline$C$ & 3.119219 & -1.329905 & 2.998981 \\
\hline$C$ & -3.342966 & -0.568267 & 2.732841 \\
\hline C & -3.402911 & -1.963285 & -1.317761 \\
\hline C & -2.788544 & 2.39944 & -2.806124 \\
\hline$C$ & 3.040341 & -2.778495 & -1.329804 \\
\hline$C$ & -2.676266 & 3.790538 & 1.244076 \\
\hline $\mathrm{H}$ & 6.31132 & 2.089271 & 1.357379 \\
\hline $\mathrm{H}$ & -5.467077 & 2.566359 & -2.464922 \\
\hline $\mathrm{H}$ & 6.226076 & 0.874006 & -2.22076 \\
\hline $\mathrm{H}$ & -5.339948 & 3.7654 & 0.902599 \\
\hline $\mathrm{H}$ & 3.80712 & 5.374513 & 2.506659 \\
\hline $\mathrm{H}$ & -2.304257 & 3.634539 & -5.15666 \\
\hline $\mathrm{H}$ & 3.64308 & 2.736083 & -5.091821 \\
\hline $\mathrm{H}$ & -2.125909 & 6.251789 & 2.271231 \\
\hline $\mathrm{H}$ & 5.813185 & 6.776451 & 2.919242 \\
\hline $\mathrm{H}$ & -3.931143 & 4.796297 & -6.62725 \\
\hline $\mathrm{H}$ & 5.612005 & 3.454787 & -6.417784 \\
\hline $\mathrm{H}$ & -3.721305 & 8.104229 & 2.694262 \\
\hline $\mathrm{H}$ & 8.333313 & 3.467832 & 1.777845 \\
\hline $\mathrm{H}$ & -7.114461 & 3.71257 & -3.928646 \\
\hline $\mathrm{H}$ & 8.211457 & 1.574247 & -3.536114 \\
\hline $\mathrm{H}$ & -6.959582 & 5.597817 & 1.341592 \\
\hline $\mathrm{H}$ & -5.970936 & -1.209593 & -1.11201 \\
\hline $\mathrm{H}$ & 5.766907 & -1.540006 & 2.626717 \\
\hline $\mathrm{H}$ & -5.943815 & 0.000507 & 2.256099 \\
\hline $\mathrm{H}$ & 5.707603 & -2.763812 & -0.967612 \\
\hline $\mathrm{H}$ & -3.492977 & -4.493989 & -2.336259 \\
\hline $\mathrm{H}$ & 2.64412 & -2.616399 & 5.361126 \\
\hline $\mathrm{H}$ & -3.331496 & -1.870122 & 5.09619 \\
\hline $\mathrm{H}$ & 2.476513 & -5.262272 & -2.273769 \\
\hline $\mathrm{H}$ & -5.503268 & -5.838522 & -2.85034 \\
\hline $\mathrm{H}$ & 4.282583 & -3.825191 & 6.776177 \\
\hline
\end{tabular}




\begin{tabular}{|c|c|c|c|}
\hline $\mathrm{H}$ & -5.285117 & -2.532128 & 6.476602 \\
\hline $\mathrm{H}$ & 4.051389 & -7.151932 & -2.597455 \\
\hline $\mathrm{H}$ & -8.004509 & -2.535685 & -1.649619 \\
\hline $\mathrm{H}$ & 7.427894 & -2.732986 & 4.035361 \\
\hline $\mathrm{H}$ & -7.912927 & -0.637401 & 3.630739 \\
\hline $\mathrm{H}$ & 7.305976 & -4.635013 & -1.301731 \\
\hline $\mathrm{H}$ & 7.932549 & 2.907101 & -5.639734 \\
\hline $\mathrm{H}$ & -6.35916 & 4.877206 & -6.014071 \\
\hline $\mathrm{H}$ & 6.698877 & -3.92824 & 6.115328 \\
\hline $\mathrm{H}$ & 6.493094 & -6.867708 & -2.099757 \\
\hline $\mathrm{H}$ & -7.608268 & -1.947475 & 5.744253 \\
\hline $\mathrm{H}$ & -6.163424 & 7.806636 & 2.215434 \\
\hline $\mathrm{H}$ & -7.794207 & -4.883949 & -2.494323 \\
\hline $\mathrm{H}$ & 8.10954 & 5.84126 & 2.54898 \\
\hline $\mathrm{H}$ & -1.850112 & -0.162942 & -2.679072 \\
\hline $\mathrm{H}$ & 2.493467 & 2.904627 & -0.800402 \\
\hline $\mathrm{H}$ & 0.993675 & 0.219014 & 4.605162 \\
\hline $\mathrm{H}$ & 0.230794 & 1.477657 & 4.005557 \\
\hline $\mathrm{H}$ & 0.631311 & -2.663044 & -3.502668 \\
\hline $\mathrm{H}$ & -0.924811 & -2.944849 & 3.398181 \\
\hline $\mathrm{H}$ & -1.728326 & 1.616835 & 2.710639 \\
\hline $\mathrm{H}$ & 1.549599 & -2.575103 & 0.99544 \\
\hline $\mathrm{H}$ & 1.23122 & 3.352707 & 3.672128 \\
\hline $\mathrm{H}$ & 0.11722 & -0.600211 & -3.875509 \\
\hline $\mathrm{H}$ & 1.256029 & 0.356432 & -4.415239 \\
\hline $\mathrm{H}$ & -0.149742 & 5.09711 & 0.158968 \\
\hline $\mathrm{H}$ & -0.019262 & 4.104442 & -2.973418 \\
\hline $\mathrm{H}$ & 0.710187 & 4.374452 & -0.964333 \\
\hline $\mathrm{O}$ & 1.238291 & 0.757227 & 1.481844 \\
\hline $\mathrm{O}$ & 3.571579 & -0.893731 & 1.897792 \\
\hline $\mathrm{O}$ & 3.522223 & -1.757521 & -0.739959 \\
\hline $\mathrm{O}$ & 3.944213 & 1.680549 & 1.074706 \\
\hline $\mathrm{O}$ & 2.589718 & 3.32612 & 1.826832 \\
\hline $\mathrm{O}$ & 0.695367 & 4.636599 & 0.021953 \\
\hline $\mathrm{O}$ & -1.43708 & 4.012131 & 1.417614 \\
\hline $\mathrm{O}$ & -1.564056 & 2.438908 & -3.145897 \\
\hline $\mathrm{O}$ & -3.179897 & 2.697096 & 0.838912 \\
\hline $\mathrm{O}$ & -3.245555 & 1.849583 & -1.755072 \\
\hline $\mathrm{O}$ & -3.580515 & 0.084888 & 1.670103 \\
\hline $\mathrm{O}$ & -3.612504 & -0.788002 & -0.885302 \\
\hline $\mathrm{O}$ & -2.266931 & -2.489395 & -1.50563 \\
\hline $\mathrm{O}$ & -2.194382 & -0.931827 & 3.140404 \\
\hline $\mathrm{O}$ & -1.145019 & -0.858218 & 0.500855 \\
\hline
\end{tabular}




\begin{tabular}{|c|c|c|c|}
\hline 0 & -1.166277 & 1.285905 & 1.995026 \\
\hline 0 & 0.406927 & 2.957712 & 3.352675 \\
\hline 0 & 0.129247 & 0.491669 & 4.25354 \\
\hline 0 & 0.329427 & 0.358244 & -4.120688 \\
\hline $\mathrm{O}$ & -0.677628 & 1.99827 & -0.407793 \\
\hline 0 & 1.835062 & 2.244626 & -0.539255 \\
\hline 0 & 2.474206 & 1.601574 & -3.180867 \\
\hline $\mathrm{O}$ & 0.697728 & 3.712392 & -2.454038 \\
\hline $\mathrm{O}$ & 3.906371 & 0.856593 & -1.597896 \\
\hline 0 & -0.359872 & -2.813371 & 2.620183 \\
\hline $\mathrm{O}$ & -0.311732 & -3.707768 & -0.293178 \\
\hline $\mathrm{O}$ & 1.243157 & -1.692445 & 0.735562 \\
\hline $\mathrm{O}$ & 1.911013 & -1.24477 & 3.389522 \\
\hline 0 & 1.843313 & -2.919351 & -1.70232 \\
\hline 0 & -0.155498 & -2.166533 & -3.230166 \\
\hline $\mathrm{O}$ & -1.242426 & 0.021997 & -1.949027 \\
\hline $\mathrm{O}$ & 1.218269 & -0.113966 & -1.325278 \\
\hline $\mathrm{Zr}$ & -2.316579 & 0.802701 & -0.009063 \\
\hline $\mathrm{Zr}$ & 0.471813 & 2.686682 & 1.26311 \\
\hline $\mathrm{Zr}$ & -0.06847 & -0.703204 & 2.280205 \\
\hline $\mathrm{Zr}$ & -0.066623 & -1.772725 & -1.141264 \\
\hline $\mathrm{Zr}$ & 2.667526 & 0.111053 & 0.112805 \\
\hline $\mathrm{Zr}$ & 0.367123 & 1.647189 & -2.174419 \\
\hline C & -3.511789 & -3.868183 & 1.570355 \\
\hline $\mathrm{H}$ & -4.052019 & -2.908347 & 1.515361 \\
\hline $\mathrm{H}$ & -3.968618 & -4.493257 & 0.788853 \\
\hline $\mathrm{Zn}$ & -1.642061 & -3.477363 & 1.111867 \\
\hline C & -3.729923 & -4.507683 & 2.93638 \\
\hline $\mathrm{H}$ & -4.796335 & -4.587329 & 3.200958 \\
\hline $\mathrm{H}$ & -3.309924 & -5.521592 & 2.985062 \\
\hline $\mathrm{H}$ & -3.258368 & -3.932125 & 3.745932 \\
\hline $\mathrm{Zn}$ & -0.93041 & -4.652898 & -1.750411 \\
\hline C & -1.318005 & -5.819236 & -3.243632 \\
\hline $\mathrm{H}$ & -1.973286 & -6.636695 & -2.910985 \\
\hline $\mathrm{H}$ & -1.909911 & -5.216567 & -3.947263 \\
\hline C & -0.083761 & -6.377339 & -3.944384 \\
\hline $\mathrm{H}$ & -0.351206 & -6.994193 & -4.814734 \\
\hline $\mathrm{H}$ & 0.574234 & -5.578978 & -4.313498 \\
\hline $\mathrm{H}$ & 0.520895 & -7.00972 & -3.280887 \\
\hline
\end{tabular}


Table S13. Structure for $\mathrm{H}_{2} \mathrm{O} / \mu \mathrm{OH}-2 \mathrm{Et}$.

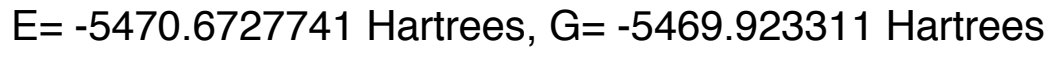

\begin{tabular}{|c|c|c|c|}
\hline Atom & $x$ & $Y$ & Z \\
\hline$C$ & 5.318851 & 4.438293 & 1.059915 \\
\hline$C$ & -6.101051 & 1.623237 & -3.022426 \\
\hline$C$ & 5.218215 & 2.459113 & -3.680811 \\
\hline$C$ & -5.978089 & 3.581657 & 1.53671 \\
\hline$C$ & 3.969445 & 4.690571 & 1.348055 \\
\hline $\mathrm{C}$ & -4.795097 & 1.879029 & -3.466644 \\
\hline$C$ & 3.853874 & 2.474986 & -4.007163 \\
\hline$C$ & -4.665876 & 4.063551 & 1.654105 \\
\hline C & 3.588093 & 5.982489 & 1.740681 \\
\hline$C$ & -4.60758 & 2.496353 & -4.712225 \\
\hline C & 3.453399 & 3.059681 & -5.217963 \\
\hline C & -4.461849 & 5.386553 & 2.072519 \\
\hline$C$ & 4.533196 & 7.002117 & 1.826987 \\
\hline C & -5.703061 & 2.877351 & -5.481162 \\
\hline$C$ & 4.390435 & 3.64418 & -6.0656 \\
\hline C & -5.546611 & 6.217582 & 2.338561 \\
\hline C & 5.88978 & 6.759415 & 1.534977 \\
\hline C & -7.018711 & 2.656351 & -5.029181 \\
\hline C & 5.759597 & 3.657514 & -5.733884 \\
\hline $\mathrm{C}$ & -6.868504 & 5.750108 & 2.200328 \\
\hline C & 6.263024 & 5.455364 & 1.156784 \\
\hline C & -7.194092 & 2.002915 & -3.794954 \\
\hline$C$ & 6.154236 & 3.036575 & -4.533172 \\
\hline$C$ & -7.060791 & 4.410926 & 1.81132 \\
\hline C & -5.530146 & -3.559442 & -0.831442 \\
\hline C & 5.83877 & -0.62683 & 3.1958 \\
\hline$C$ & -5.48029 & -1.607593 & 3.74143 \\
\hline$C$ & 5.753477 & -2.611691 & -1.568895 \\
\hline C & -4.179175 & -3.829938 & -1.091674 \\
\hline$C$ & 4.538042 & -0.86151 & 3.666956 \\
\hline$C$ & -4.110609 & -1.653838 & 4.040606 \\
\hline$C$ & 4.438867 & -3.081572 & -1.712647 \\
\hline C & -3.813003 & -5.107578 & -1.540896 \\
\hline C & 4.370714 & -1.488272 & 4.911388 \\
\hline $\mathrm{C}$ & -3.696107 & -2.222094 & 5.254375 \\
\hline$C$ & 4.233216 & -4.420691 & -2.078522 \\
\hline$C$ & -4.774178 & -6.101895 & -1.695727 \\
\hline C & 5.477105 & -1.904408 & 5.646932 \\
\hline C & -4.629903 & -2.761291 & 6.13397 \\
\hline
\end{tabular}




\begin{tabular}{|c|c|c|c|}
\hline C & 5.31673 & -5.273689 & -2.275532 \\
\hline C & -6.130397 & -5.847577 & -1.413287 \\
\hline C & 6.786762 & -1.700708 & 5.168825 \\
\hline C & -6.004578 & -2.75169 & 5.82723 \\
\hline C & 6.639803 & -4.81393 & -2.124684 \\
\hline C & -6.490648 & -4.552144 & -0.99665 \\
\hline C & 6.943491 & -1.034721 & 3.937558 \\
\hline C & -6.411746 & -2.146076 & 4.623796 \\
\hline C & 6.834626 & -3.463297 & -1.776709 \\
\hline C & 2.86186 & 1.901918 & -3.050922 \\
\hline C & 2.955943 & 3.613674 & 1.168742 \\
\hline C & 3.368471 & -0.470141 & 2.823423 \\
\hline C & -3.104672 & -1.125598 & 3.079158 \\
\hline C & -3.15503 & -2.76423 & -0.876381 \\
\hline C & -3.622127 & 1.521597 & -2.623441 \\
\hline C & 3.288121 & -2.183572 & -1.407756 \\
\hline C & -3.517389 & 3.167401 & 1.330424 \\
\hline $\mathrm{H}$ & 5.607197 & 3.436451 & 0.749386 \\
\hline $\mathrm{H}$ & -6.240077 & 1.1358 & -2.059902 \\
\hline $\mathrm{H}$ & 5.525403 & 1.99885 & -2.744511 \\
\hline $\mathrm{H}$ & -6.129801 & 2.551334 & 1.223309 \\
\hline $\mathrm{H}$ & 2.53902 & 6.178737 & 1.951376 \\
\hline $\mathrm{H}$ & -3.59382 & 2.675669 & -5.063113 \\
\hline $\mathrm{H}$ & 2.394502 & 3.073542 & -5.468379 \\
\hline $\mathrm{H}$ & -3.443144 & 5.75139 & 2.187558 \\
\hline $\mathrm{H}$ & 4.215716 & 8.001523 & 2.121517 \\
\hline $\mathrm{H}$ & -5.538969 & 3.363259 & -6.442061 \\
\hline $\mathrm{H}$ & 4.055832 & 4.108075 & -6.992546 \\
\hline $\mathrm{H}$ & -5.36861 & 7.243954 & 2.656603 \\
\hline $\mathrm{H}$ & 7.30587 & 5.24004 & 0.927251 \\
\hline $\mathrm{H}$ & -8.200877 & 1.803974 & -3.429948 \\
\hline $\mathrm{H}$ & 7.208349 & 3.019407 & -4.258717 \\
\hline $\mathrm{H}$ & -8.072712 & 4.020383 & 1.710753 \\
\hline $\mathrm{H}$ & -5.806916 & -2.564184 & -0.491404 \\
\hline $\mathrm{H}$ & 5.963986 & -0.139508 & 2.231481 \\
\hline $\mathrm{H}$ & -5.79528 & -1.154324 & 2.804133 \\
\hline $\mathrm{H}$ & 5.907633 & -1.576277 & -1.272803 \\
\hline $\mathrm{H}$ & -2.768626 & -5.328958 & -1.749926 \\
\hline $\mathrm{H}$ & 3.361746 & -1.673915 & 5.274971 \\
\hline $\mathrm{H}$ & -2.634379 & -2.237293 & 5.489875 \\
\hline $\mathrm{H}$ & 3.212825 & -4.791322 & -2.16897 \\
\hline $\mathrm{H}$ & -4.466542 & -7.090018 & -2.037495 \\
\hline $\mathrm{H}$ & 5.324789 & -2.40774 & 6.600891 \\
\hline
\end{tabular}




\begin{tabular}{|c|c|c|c|}
\hline $\mathrm{H}$ & -4.290769 & -3.205483 & 7.068992 \\
\hline $\mathrm{H}$ & 5.135198 & -6.314472 & -2.54047 \\
\hline $\mathrm{H}$ & -7.534843 & -4.326473 & -0.784509 \\
\hline $\mathrm{H}$ & 7.944646 & -0.852161 & 3.548936 \\
\hline $\mathrm{H}$ & -7.470825 & -2.109513 & 4.37147 \\
\hline $\mathrm{H}$ & 7.847036 & -3.080437 & -1.653816 \\
\hline $\mathrm{H}$ & 6.490887 & 4.120657 & -6.392172 \\
\hline $\mathrm{H}$ & -7.873983 & 2.963435 & -5.626506 \\
\hline $\mathrm{H}$ & 7.64964 & -2.034778 & 5.740075 \\
\hline $\mathrm{H}$ & 7.484629 & -5.482463 & -2.272469 \\
\hline $\mathrm{H}$ & -6.732545 & -3.18182 & 6.511205 \\
\hline $\mathrm{H}$ & -7.715434 & 6.4008 & 2.405121 \\
\hline $\mathrm{H}$ & -6.880369 & -6.626305 & -1.530229 \\
\hline $\mathrm{H}$ & 6.627123 & 7.556171 & 1.599924 \\
\hline $\mathrm{H}$ & -2.154586 & -0.777514 & -2.435887 \\
\hline $\mathrm{H}$ & 1.582791 & 3.249133 & -1.099842 \\
\hline $\mathrm{H}$ & 1.085908 & 0.669494 & 4.509599 \\
\hline $\mathrm{H}$ & 0.02292 & 1.693006 & 3.932208 \\
\hline $\mathrm{H}$ & 0.825924 & -2.773042 & -3.224667 \\
\hline $\mathrm{H}$ & -0.407632 & -4.025935 & 0.466834 \\
\hline $\mathrm{H}$ & -0.08503 & -3.207447 & 3.336417 \\
\hline $\mathrm{H}$ & -2.008049 & 1.357073 & 2.820266 \\
\hline $\mathrm{H}$ & 0.55814 & 3.743433 & 3.440066 \\
\hline $\mathrm{H}$ & -0.268099 & -0.835414 & -3.813211 \\
\hline $\mathrm{H}$ & 0.7106 & 0.237329 & -4.399074 \\
\hline $\mathrm{H}$ & -1.427513 & 4.917204 & -0.022759 \\
\hline $\mathrm{H}$ & -1.31524 & 3.803114 & -3.116979 \\
\hline $\mathrm{H}$ & -0.50421 & 4.341476 & -1.175005 \\
\hline $\mathrm{O}$ & 0.965356 & 1.085566 & 1.391772 \\
\hline $\mathrm{O}$ & 3.631772 & -0.013504 & 1.669795 \\
\hline $\mathrm{O}$ & 3.576733 & -1.033723 & -0.948729 \\
\hline $\mathrm{O}$ & 3.363962 & 2.511828 & 0.691831 \\
\hline O & 1.750535 & 3.885625 & 1.478549 \\
\hline $\mathrm{O}$ & -0.51138 & 4.648234 & -0.205352 \\
\hline $\mathrm{O}$ & -2.349005 & 3.658167 & 1.383302 \\
\hline $\mathrm{O}$ & -2.465139 & 1.800984 & -3.072668 \\
\hline $\mathrm{O}$ & -3.806762 & 1.970171 & 1.020741 \\
\hline $\mathrm{O}$ & -3.869917 & 0.956178 & -1.514028 \\
\hline $\mathrm{O}$ & -3.567763 & -0.592396 & 2.020036 \\
\hline $\mathrm{O}$ & -3.585084 & -1.631854 & -0.509843 \\
\hline $\mathrm{O}$ & -1.934978 & -3.045328 & -1.101837 \\
\hline $\mathrm{O}$ & -1.876747 & -1.237104 & 3.381532 \\
\hline $\mathrm{O}$ & -1.113851 & -1.044325 & 0.676757 \\
\hline
\end{tabular}




$\begin{array}{lrrr}\mathrm{O} & -1.452858 & 1.107671 & 2.067428 \\ \mathrm{O} & -0.186399 & 3.169277 & 3.208326 \\ \mathrm{O} & 0.156972 & 0.729916 & 4.228432 \\ \mathrm{O} & -0.21551 & 0.097699 & -4.13053 \\ \mathrm{O} & -1.303611 & 1.746866 & -0.406068 \\ \mathrm{O} & 1.068204 & 2.503394 & -0.758973 \\ \mathrm{O} & 1.642288 & 1.84974 & -3.421592 \\ \mathrm{O} & -0.496203 & 3.596206 & -2.644139 \\ \mathrm{O} & 3.307178 & 1.529355 & -1.922944 \\ \mathrm{O} & 0.39722 & -2.691391 & 2.679694 \\ \mathrm{O} & 0.324464 & -3.852191 & -0.14901 \\ \mathrm{O} & 1.424464 & -1.348276 & 0.73063 \\ \mathrm{O} & 2.205066 & -0.624331 & 3.311598 \\ \mathrm{O} & 2.117288 & -2.615568 & -1.617827 \\ \mathrm{O} & -0.089663 & -2.667216 & -2.91507 \\ \mathrm{O} & -1.522806 & -0.402766 & -1.807261 \\ \mathrm{O} & 0.943531 & 0.027492 & -1.455682 \\ \mathrm{Zr} & -2.623919 & 0.27546 & 0.206217 \\ \mathrm{Zr} & -0.207695 & 2.775203 & 1.144479 \\ \mathrm{Zr} & 0.086485 & -0.611765 & 2.32543 \\ \mathrm{Zr} & 0.069079 & -1.754787 & -0.892169 \\ \mathrm{Zr} & 2.374914 & 0.615462 & -0.058063 \\ \mathrm{Zr} & -0.348108 & 1.538081 & -2.234842 \\ \mathrm{C} & 3.422936 & -4.480032 & 1.494656 \\ \mathrm{H} & 3.139155 & -5.243654 & 2.23448 \\ \mathrm{H} & 3.413088 & -4.9978 & 0.523416 \\ \mathrm{Zn} & 1.965081 & -3.179112 & 1.387578 \\ \mathrm{Zn} & -0.392536 & -4.525366 & -1.997778 \\ \mathrm{C} & -0.601804 & -6.303012 & -2.797976 \\ \mathrm{H} & 0.405948 & -6.717646 & -2.944582 \\ \mathrm{H} & -1.081449 & -6.964996 & -2.061485 \\ \mathrm{C} & 4.826624 & -3.967948 & 1.795333 \\ \mathrm{H} & 5.590159 & -4.75439 & 1.680753 \\ \mathrm{H} & 5.121918 & -3.14454 & 1.130008 \\ \mathrm{H} & 4.912441 & -3.586926 & 2.821579 \\ \mathrm{H} & -1.373842 & -6.32893 & -4.112434 \\ \mathrm{H} & -2.398887 & -5.948929 & -4.000013 \\ \mathrm{H} & & -5.708421 & -4.88104\end{array}$


Table S14. Structure for one $\mathrm{ZnEt}$ group bound to $\mu \mathrm{OH}$ site and another to the $\mathrm{OH}$ site. $E=-5470.65429784$ Hartrees, $G=-5469.904521$ Hartrees

\begin{tabular}{|c|c|c|c|}
\hline Atom & $\mathrm{X}$ & $Y$ & Z \\
\hline $\mathrm{C}$ & 5.266901 & -3.1085 & -3.2087 \\
\hline C & -6.015901 & -3.276328 & 2.051933 \\
\hline C & 5.314772 & -4.18094 & 1.816143 \\
\hline C & -6.03596 & -2.289846 & -2.812533 \\
\hline C & 3.908566 & -3.170653 & -3.553263 \\
\hline C & -4.698284 & -3.721301 & 2.236551 \\
\hline C & 3.960636 & -4.39875 & 2.111551 \\
\hline C & -4.729624 & -2.600944 & -3.218238 \\
\hline $\mathrm{C}$ & 3.510937 & -4.014568 & -4.601224 \\
\hline C & -4.476051 & -4.935152 & 2.903621 \\
\hline C & 3.594276 & -5.574077 & 2.784213 \\
\hline $\mathrm{C}$ & -4.543048 & -3.447608 & -4.320685 \\
\hline $\mathrm{C}$ & 4.449271 & -4.790529 & -5.277777 \\
\hline $\mathrm{C}$ & -5.549555 & -5.701263 & 3.347641 \\
\hline C & 4.553951 & -6.524033 & 3.123003 \\
\hline C & -5.638316 & -3.994509 & -4.983541 \\
\hline $\mathrm{C}$ & 5.814839 & -4.738326 & -4.935738 \\
\hline $\mathrm{C}$ & -6.877248 & -5.280631 & 3.137009 \\
\hline C & 5.912569 & -6.327181 & 2.806426 \\
\hline $\mathrm{C}$ & -6.953767 & -3.707215 & -4.568701 \\
\hline C & 6.204008 & -3.87632 & -3.892481 \\
\hline $\mathrm{C}$ & -7.086866 & -4.043405 & 2.499531 \\
\hline C & 6.273594 & -5.127821 & 2.162831 \\
\hline C & -7.129424 & -2.830305 & -3.481556 \\
\hline C & -5.491286 & 2.239017 & 3.187168 \\
\hline C & 5.741805 & 2.278526 & -2.093963 \\
\hline C & -5.584767 & 3.23806 & -1.68306 \\
\hline C & 5.805732 & 1.197219 & 2.953472 \\
\hline C & -4.131707 & 2.333429 & 3.51842 \\
\hline C & 4.428492 & 2.721531 & -2.312973 \\
\hline $\mathrm{C}$ & -4.224375 & 3.465403 & -1.939074 \\
\hline C & 4.497641 & 1.4835 & 3.374124 \\
\hline C & -3.747962 & 3.133059 & 4.604908 \\
\hline C & 4.226537 & 3.942754 & -2.974202 \\
\hline C & -3.84414 & 4.629123 & -2.623852 \\
\hline C & 4.308023 & 2.373365 & 4.442411 \\
\hline C & -4.700491 & 3.847779 & 5.324775 \\
\hline C & 5.312081 & 4.718896 & -3.371063 \\
\hline
\end{tabular}




\begin{tabular}{|c|c|c|c|}
\hline C & -4.801701 & 5.560076 & -3.014767 \\
\hline C & 5.400087 & 2.977465 & 5.061185 \\
\hline C & -6.06543 & 3.780855 & 4.983739 \\
\hline C & 6.634625 & 4.297524 & -3.128972 \\
\hline C & -6.166659 & 5.358501 & -2.731634 \\
\hline C & 6.716369 & 2.703719 & 4.640336 \\
\hline C & -6.442713 & 2.948123 & 3.913355 \\
\hline C & 6.825463 & 3.051403 & -2.500573 \\
\hline C & -6.54007 & 4.170016 & -2.076744 \\
\hline C & 6.895738 & 1.794122 & 3.580277 \\
\hline C & 2.942851 & -3.396982 & 1.67815 \\
\hline C & 2.905093 & -2.4013 & -2.766045 \\
\hline C & 3.283725 & 1.903531 & -1.81161 \\
\hline C & -3.192282 & 2.498038 & -1.47632 \\
\hline C & -3.121125 & 1.59101 & 2.709676 \\
\hline C & -3.549474 & -2.931044 & 1.715735 \\
\hline C & 3.336078 & 0.902471 & 2.642461 \\
\hline C & -3.568636 & -2.032375 & -2.472612 \\
\hline $\mathrm{H}$ & 5.567702 & -2.458689 & -2.389816 \\
\hline $\mathrm{H}$ & -6.181088 & -2.328471 & 1.544398 \\
\hline $\mathrm{H}$ & 5.595592 & -3.265091 & 1.301186 \\
\hline $\mathrm{H}$ & -6.173527 & -1.62495 & -1.962965 \\
\hline $\mathrm{H}$ & 2.455306 & -4.070579 & -4.857942 \\
\hline $\mathrm{H}$ & -3.452708 & -5.266852 & 3.064034 \\
\hline $\mathrm{H}$ & 2.54329 & -5.7435 & 3.009665 \\
\hline $\mathrm{H}$ & -3.52939 & -3.667966 & -4.6494 \\
\hline $\mathrm{H}$ & 4.119514 & -5.449172 & -6.080179 \\
\hline $\mathrm{H}$ & -5.358791 & -6.645014 & 3.857029 \\
\hline $\mathrm{H}$ & 4.245296 & -7.437541 & 3.629577 \\
\hline $\mathrm{H}$ & -5.473866 & -4.655306 & -5.833627 \\
\hline $\mathrm{H}$ & 7.254013 & -3.816018 & -3.608675 \\
\hline $\mathrm{H}$ & -8.103473 & -3.686122 & 2.339944 \\
\hline $\mathrm{H}$ & 7.319193 & -4.943244 & 1.918786 \\
\hline $\mathrm{H}$ & -8.136519 & -2.58142 & -3.149098 \\
\hline $\mathrm{H}$ & -5.781847 & 1.60688 & 2.351421 \\
\hline $\mathrm{H}$ & 5.893496 & 1.330176 & -1.583364 \\
\hline $\mathrm{H}$ & -5.873071 & 2.327813 & -1.16206 \\
\hline $\mathrm{H}$ & 5.947535 & 0.513655 & 2.118988 \\
\hline $\mathrm{H}$ & -2.695862 & 3.181122 & 4.878664 \\
\hline $\mathrm{H}$ & 3.207906 & 4.288029 & -3.144124 \\
\hline $\mathrm{H}$ & -2.794786 & 4.785503 & -2.859739 \\
\hline $\mathrm{H}$ & 3.292498 & 2.605288 & 4.757563 \\
\hline $\mathrm{H}$ & -4.384498 & 4.468356 & 6.162303 \\
\hline
\end{tabular}




\begin{tabular}{|c|c|c|c|}
\hline $\mathrm{H}$ & 5.133473 & 5.672738 & -3.865768 \\
\hline $\mathrm{H}$ & -4.486397 & 6.46152 & -3.539751 \\
\hline $\mathrm{H}$ & 5.230793 & 3.677033 & 5.878783 \\
\hline $\mathrm{H}$ & -7.494294 & 2.86692 & 3.640736 \\
\hline $\mathrm{H}$ & 7.836885 & 2.694146 & -2.311352 \\
\hline $\mathrm{H}$ & -7.59108 & 3.981237 & -1.862177 \\
\hline $\mathrm{H}$ & 7.90279 & 1.562672 & 3.235268 \\
\hline $\mathrm{H}$ & 6.661406 & -7.072506 & 3.064113 \\
\hline $\mathrm{H}$ & -7.715549 & -5.884785 & 3.476013 \\
\hline $\mathrm{H}$ & 7.481162 & 4.909179 & -3.431621 \\
\hline $\mathrm{H}$ & 7.568039 & 3.179489 & 5.120861 \\
\hline $\mathrm{H}$ & -6.912772 & 6.091208 & -3.029997 \\
\hline $\mathrm{H}$ & -7.808889 & -4.13773 & -5.08462 \\
\hline $\mathrm{H}$ & -6.809023 & 4.342699 & 5.54427 \\
\hline $\mathrm{H}$ & 6.546947 & -5.346322 & -5.462181 \\
\hline $\mathrm{H}$ & -2.033601 & -0.957276 & 2.87762 \\
\hline $\mathrm{H}$ & 1.595559 & -3.42871 & -0.667696 \\
\hline $\mathrm{H}$ & 0.937489 & 1.904254 & -3.751938 \\
\hline $\mathrm{H}$ & -0.113413 & 0.724457 & -3.834062 \\
\hline $\mathrm{H}$ & 0.858368 & 0.132551 & 4.613406 \\
\hline $\mathrm{H}$ & -0.055521 & 3.395918 & 1.28669 \\
\hline $\mathrm{H}$ & -0.728305 & 3.307249 & 2.712815 \\
\hline $\mathrm{H}$ & -2.099797 & 0.315947 & -2.685637 \\
\hline $\mathrm{H}$ & 0.436949 & -1.224591 & -4.626533 \\
\hline $\mathrm{H}$ & -0.073878 & -1.682179 & 3.913138 \\
\hline $\mathrm{H}$ & 0.739236 & -3.040108 & 3.799035 \\
\hline $\mathrm{H}$ & -1.450539 & -4.219102 & -2.410675 \\
\hline $\mathrm{H}$ & -1.247761 & -5.059602 & 0.749352 \\
\hline $\mathrm{H}$ & -0.492206 & -4.388652 & -1.157407 \\
\hline $\mathrm{O}$ & 0.936148 & -0.235644 & -1.469587 \\
\hline $\mathrm{O}$ & 3.579184 & 0.875559 & -1.134467 \\
\hline $\mathrm{O}$ & 3.603718 & 0.198071 & 1.621019 \\
\hline 0 & 3.333141 & -1.761435 & -1.758031 \\
\hline $\mathrm{O}$ & 1.69025 & -2.468593 & -3.142376 \\
\hline 0 & -0.528454 & -4.089844 & -2.131645 \\
\hline $\mathrm{O}$ & -2.404389 & -2.389016 & -2.831595 \\
\hline $\mathrm{O}$ & -2.385981 & -3.412745 & 1.875423 \\
\hline $\mathrm{O}$ & -3.841752 & -1.232021 & -1.526041 \\
\hline $\mathrm{O}$ & -3.833243 & -1.837152 & 1.138018 \\
\hline $\mathrm{O}$ & -3.613485 & 1.444881 & -0.916296 \\
\hline $\mathrm{O}$ & -3.576988 & 0.855971 & 1.777569 \\
\hline $\mathrm{O}$ & -1.899339 & 1.724411 & 3.020238 \\
\hline 0 & -1.965275 & 2.810417 & -1.653303 \\
\hline
\end{tabular}




$\begin{array}{lrrr}\mathrm{O} & -1.110156 & 1.062817 & 0.442184 \\ \mathrm{O} & -1.520585 & 0.106582 & -1.93925 \\ \mathrm{O} & -0.299809 & -0.901885 & -4.087517 \\ \mathrm{O} & 0.017013 & 1.686844 & -3.528461 \\ \mathrm{O} & -0.139142 & -2.660323 & 3.62665 \\ \mathrm{O} & -1.304377 & -1.835328 & -0.295293 \\ \mathrm{O} & 1.081915 & -2.625159 & -0.502156 \\ \mathrm{O} & 1.735604 & -3.595841 & 2.025056 \\ \mathrm{O} & -0.435647 & -4.618091 & 0.461343 \\ \mathrm{O} & 3.358869 & -2.435014 & 0.961909 \\ \mathrm{O} & 0.31267 & 3.596959 & -0.376141 \\ \mathrm{O} & 0.109688 & 3.136758 & 2.243363 \\ \mathrm{O} & 1.423606 & 1.384903 & 0.482162 \\ \mathrm{O} & 2.104631 & 2.318077 & -2.059134 \\ \mathrm{O} & 2.171274 & 1.165257 & 3.079487 \\ \mathrm{O} & 0.041576 & -0.063786 & 4.132326 \\ \mathrm{O} & -1.484101 & -0.87396 & 2.085117 \\ \mathrm{O} & 0.952169 & -0.969876 & 1.446897 \\ \mathrm{Zr} & -2.624025 & -0.295318 & 0.103905 \\ \mathrm{Zr} & -0.259823 & -1.780842 & -2.174051 \\ \mathrm{Zr} & 0.015306 & 1.644989 & -1.18372 \\ \mathrm{Zr} & 0.100341 & 0.760404 & 2.186286 \\ \mathrm{Zr} & 2.364118 & -0.663644 & -0.027294 \\ \mathrm{Zr} & -0.289837 & -2.694517 & 1.311667 \\ \mathrm{C} & 3.539347 & 4.414538 & 1.217132 \\ \mathrm{H} & 3.228505 & 5.43374 & 0.943903 \\ \mathrm{H} & 3.40563 & 4.347134 & 2.308336 \\ \mathrm{Zn} & 2.214482 & 3.169898 & 0.517842 \\ \mathrm{Zn} & -0.588012 & 4.979734 & -1.135926 \\ \mathrm{C} & -1.255692 & 6.676288 & -1.782815 \\ \mathrm{H} & -1.358571 & 6.60387 & -2.875466 \\ \mathrm{H} & -2.277406 & 6.798004 & -1.39683 \\ \mathrm{H} & 5.004294 & 4.197861 & 0.859162 \\ \mathrm{H} & 5.209594 & 4.447152 & -0.189804 \\ \mathrm{H} & 5.673172 & 4.818765 & 1.475566 \\ \mathrm{H} & -0.319255 & 3.155347 & 1.004041 \\ \mathrm{H} & 0.624435 & 7.798826 & -1.811472 \\ \mathrm{H} & -301588 & 8.002835 & -0.326246\end{array}$


Table S15. Structure for $\mathrm{H}_{2} \mathrm{O} / \mathrm{OH}-2 \mathrm{Et}$.

$E=-5470.62737032$ Hartrees, $G=-5469.876479$ Hartrees

\begin{tabular}{|c|c|c|c|}
\hline Atom & $x$ & $Y$ & Z \\
\hline C & -6.309864 & 1.270787 & -3.006002 \\
\hline$C$ & 4.533273 & 4.952577 & 1.88018 \\
\hline C & -6.511703 & 2.261187 & 2.031838 \\
\hline$C$ & 4.687532 & 4.034662 & -2.99529 \\
\hline$C$ & -5.052505 & 1.756404 & -3.394443 \\
\hline C & 3.150065 & 4.961711 & 2.114355 \\
\hline C & -5.284222 & 2.891654 & 2.285207 \\
\hline$C$ & 3.335859 & 3.921727 & -3.353276 \\
\hline C & -4.976791 & 2.685134 & -4.443374 \\
\hline C & 2.582896 & 6.043019 & 2.80494 \\
\hline C & -5.280525 & 4.120832 & 2.961367 \\
\hline C & 2.854334 & 4.670139 & -4.43726 \\
\hline$C$ & -6.134428 & 3.129674 & -5.077858 \\
\hline C & 3.37767 & 7.105826 & 3.22359 \\
\hline C & -6.476648 & 4.721289 & 3.344656 \\
\hline C & 3.69889 & 5.534449 & -5.12848 \\
\hline C & -7.401856 & 2.651249 & -4.691564 \\
\hline C & 4.761895 & 7.123033 & 2.963416 \\
\hline C & -7.715703 & 4.109382 & 3.070554 \\
\hline $\mathrm{C}$ & 5.052077 & 5.672816 & -4.761356 \\
\hline C & -7.464046 & 1.708003 & -3.64786 \\
\hline C & 5.325465 & 6.015487 & 2.302525 \\
\hline C & -7.705773 & 2.858972 & 2.422953 \\
\hline C & 5.532168 & 4.892321 & -3.69269 \\
\hline C & 5.802847 & -0.45225 & 2.956812 \\
\hline C & -5.034019 & -3.996555 & -1.949757 \\
\hline C & 6.030506 & -1.359037 & -1.927066 \\
\hline C & -5.253071 & -3.002812 & 3.111306 \\
\hline C & 4.553758 & -0.968902 & 3.331799 \\
\hline C & -3.656737 & -4.005079 & -2.218136 \\
\hline C & 4.801439 & -2.00057 & -2.141141 \\
\hline C & -3.906978 & -2.865806 & 3.484492 \\
\hline C & 4.478877 & -1.851166 & 4.419212 \\
\hline C & -3.106298 & -5.099764 & -2.902296 \\
\hline C & 4.780543 & -3.223071 & -2.828673 \\
\hline C & -3.410255 & -3.65418 & 4.533676 \\
\hline C & 5.632386 & -2.233305 & 5.097268 \\
\hline C & -3.907676 & -6.175985 & -3.273571 \\
\hline C & 5.966858 & -3.806044 & -3.263661 \\
\hline
\end{tabular}




\begin{tabular}{|c|c|c|c|}
\hline C & -4.235428 & -4.571696 & 5.179949 \\
\hline C & 6.894643 & -1.74112 & 4.712181 \\
\hline C & -5.286123 & -6.190955 & -2.982118 \\
\hline C & 7.209256 & -3.187589 & -3.023113 \\
\hline C & -5.585378 & -4.723176 & 4.806663 \\
\hline C & 6.953755 & -0.829336 & 3.641352 \\
\hline C & -5.83498 & -5.069052 & -2.330802 \\
\hline C & 7.214891 & -1.943648 & -2.364788 \\
\hline C & -6.078257 & -3.912876 & 3.76563 \\
\hline C & -4.020149 & 2.260472 & 1.804698 \\
\hline C & -3.83124 & 1.338281 & -2.651505 \\
\hline C & -2.808758 & -2.87078 & -1.743777 \\
\hline C & 3.535881 & -1.40666 & -1.631458 \\
\hline C & 3.334007 & -0.578787 & 2.567459 \\
\hline C & 2.288554 & 3.852626 & 1.621062 \\
\hline C & -3.01271 & -1.948169 & 2.72279 \\
\hline C & 2.438663 & 3.016102 & -2.577697 \\
\hline $\mathrm{H}$ & -6.363169 & 0.555769 & -2.18846 \\
\hline $\mathrm{H}$ & 4.968275 & 4.105368 & 1.354947 \\
\hline $\mathrm{H}$ & -6.510404 & 1.305189 & 1.513956 \\
\hline $\mathrm{H}$ & 5.056442 & 3.443031 & -2.160839 \\
\hline $\mathrm{H}$ & -4.001851 & 3.069489 & -4.73463 \\
\hline $\mathrm{H}$ & 1.513658 & 6.036745 & 3.003092 \\
\hline $\mathrm{H}$ & -4.328226 & 4.61039 & 3.154406 \\
\hline $\mathrm{H}$ & 1.811689 & 4.562887 & -4.728814 \\
\hline $\mathrm{H}$ & -6.056208 & 3.860016 & -5.881831 \\
\hline $\mathrm{H}$ & 2.919259 & 7.940697 & 3.751761 \\
\hline $\mathrm{H}$ & -6.451627 & 5.683877 & 3.853287 \\
\hline $\mathrm{H}$ & 3.305375 & 6.112355 & -5.963392 \\
\hline $\mathrm{H}$ & -8.431151 & 1.320015 & -3.330998 \\
\hline $\mathrm{H}$ & 6.396276 & 5.994844 & 2.104853 \\
\hline $\mathrm{H}$ & -8.64865 & 2.356266 & 2.211534 \\
\hline $\mathrm{H}$ & 6.577581 & 4.970191 & -3.397334 \\
\hline $\mathrm{H}$ & 5.852776 & 0.237997 & 2.117912 \\
\hline $\mathrm{H}$ & -5.457764 & -3.142533 & -1.42705 \\
\hline $\mathrm{H}$ & 6.037748 & -0.408706 & -1.397881 \\
\hline $\mathrm{H}$ & -5.63246 & -2.393235 & 2.294642 \\
\hline $\mathrm{H}$ & 3.504663 & -2.241878 & 4.707419 \\
\hline $\mathrm{H}$ & -2.038675 & -5.10522 & -3.113448 \\
\hline $\mathrm{H}$ & 3.826708 & -3.701962 & -3.033298 \\
\hline $\mathrm{H}$ & -2.365707 & -3.550109 & 4.820303 \\
\hline $\mathrm{H}$ & 5.556673 & -2.929588 & 5.931196 \\
\hline $\mathrm{H}$ & -3.457492 & -7.02363 & -3.788287 \\
\hline
\end{tabular}




\begin{tabular}{|c|c|c|c|}
\hline $\mathrm{H}$ & 5.930719 & -4.760399 & -3.788028 \\
\hline $\mathrm{H}$ & -3.827231 & -5.18211 & 5.984138 \\
\hline $\mathrm{H}$ & 7.916663 & -0.423743 & 3.333433 \\
\hline $\mathrm{H}$ & -6.900779 & -5.045331 & -2.1077 \\
\hline $\mathrm{H}$ & 8.160753 & -1.435942 & -2.181723 \\
\hline $\mathrm{H}$ & -7.119664 & -4.004685 & 3.460176 \\
\hline $\mathrm{H}$ & -8.65029 & 4.581644 & 3.363274 \\
\hline $\mathrm{H}$ & 5.38042 & 7.958327 & 3.282771 \\
\hline $\mathrm{H}$ & -5.909242 & -7.035302 & -3.266432 \\
\hline $\mathrm{H}$ & -6.227787 & -5.44179 & 5.30971 \\
\hline $\mathrm{H}$ & 8.136073 & -3.649272 & -3.354813 \\
\hline $\mathrm{H}$ & 5.710297 & 6.350794 & -5.299319 \\
\hline $\mathrm{H}$ & 7.796625 & -2.044473 & 5.238433 \\
\hline $\mathrm{H}$ & -8.305585 & 3.000159 & -5.185358 \\
\hline $\mathrm{H}$ & 1.545937 & 1.499894 & 2.785402 \\
\hline $\mathrm{H}$ & -2.8188 & 2.730135 & -0.575846 \\
\hline $\mathrm{H}$ & -0.650883 & -2.129129 & -3.81129 \\
\hline $\mathrm{H}$ & -0.024913 & -0.672898 & -3.891305 \\
\hline $\mathrm{H}$ & -0.742447 & -0.416549 & 4.744659 \\
\hline $\mathrm{H}$ & -0.146561 & -3.535351 & 2.17936 \\
\hline $\mathrm{H}$ & 1.786262 & 0.312285 & -2.753318 \\
\hline $\mathrm{H}$ & -1.49713 & -2.716753 & 0.568546 \\
\hline $\mathrm{H}$ & -1.184427 & 0.997494 & -4.60705 \\
\hline $\mathrm{H}$ & -0.53967 & 1.597454 & 3.949733 \\
\hline $\mathrm{H}$ & -1.738069 & 2.637382 & 3.852778 \\
\hline $\mathrm{H}$ & -0.251541 & 4.401978 & -2.410629 \\
\hline $\mathrm{H}$ & -0.597247 & 5.120581 & 0.777344 \\
\hline $\mathrm{H}$ & -1.160983 & 4.263427 & -1.11371 \\
\hline 0 & -1.233737 & -0.118389 & -1.422616 \\
\hline $\mathrm{O}$ & -3.380528 & -2.004201 & -1.014054 \\
\hline $\mathrm{O}$ & -3.517471 & -1.37888 & 1.707166 \\
\hline $\mathrm{O}$ & -4.008561 & 0.594607 & -1.638486 \\
\hline 0 & -2.709989 & 1.77468 & -3.06343 \\
\hline $\mathrm{O}$ & -1.075501 & 3.993334 & -2.09495 \\
\hline $\mathrm{O}$ & 1.20785 & 2.987608 & -2.892443 \\
\hline $\mathrm{O}$ & 1.038966 & 3.936424 & 1.832908 \\
\hline $\mathrm{O}$ & 2.975955 & 2.329948 & -1.652912 \\
\hline $\mathrm{O}$ & 2.873697 & 2.900067 & 1.016533 \\
\hline $\mathrm{O}$ & 3.624203 & -0.293365 & -1.036666 \\
\hline $\mathrm{O}$ & 3.50338 & 0.2538 & 1.617939 \\
\hline 0 & 2.229688 & -1.082204 & 2.921484 \\
\hline $\mathrm{O}$ & 2.461075 & -2.060806 & -1.835867 \\
\hline $\mathrm{O}$ & 1.178454 & -0.797918 & 0.388542 \\
\hline
\end{tabular}




\begin{tabular}{|c|c|c|c|}
\hline 0 & 1.181047 & 0.317016 & -1.997634 \\
\hline 0 & -0.367753 & 0.909269 & -4.09429 \\
\hline 0 & 0.162038 & -1.636506 & -3.606803 \\
\hline 0 & -0.793649 & 2.539809 & 3.643764 \\
\hline $\mathrm{O}$ & 0.430675 & 2.084853 & -0.322397 \\
\hline 0 & -2.092558 & 2.104296 & -0.440244 \\
\hline $\mathrm{O}$ & -2.921644 & 2.812259 & 2.119184 \\
\hline 0 & -1.236296 & 4.447695 & 0.500606 \\
\hline 0 & -4.146847 & 1.219562 & 1.086812 \\
\hline 0 & 0.674933 & -3.545425 & -0.543588 \\
\hline $\mathrm{O}$ & 0.574718 & -2.933019 & 2.409245 \\
\hline 0 & -1.15399 & -1.815833 & 0.491545 \\
\hline 0 & -1.58368 & -2.864657 & -2.078275 \\
\hline 0 & -1.822834 & -1.817321 & 3.139686 \\
\hline $\mathrm{O}$ & -0.125712 & 0.054573 & 4.166931 \\
\hline 0 & 0.99533 & 1.227315 & 2.036902 \\
\hline 0 & -1.36037 & 0.535925 & 1.458994 \\
\hline $\mathrm{Zr}$ & 2.168588 & 1.029612 & 0.012975 \\
\hline $\mathrm{Zr}$ & -0.608234 & 1.719979 & -2.164026 \\
\hline $\mathrm{Zr}$ & 0.235323 & -1.659841 & -1.28567 \\
\hline $\mathrm{Zr}$ & 0.025548 & -0.828215 & 2.266227 \\
\hline $\mathrm{Zr}$ & -2.702012 & -0.127021 & 0.038919 \\
\hline $\mathrm{Zr}$ & -0.738558 & 2.573158 & 1.332667 \\
\hline$C$ & 3.910285 & -3.483358 & 0.86016 \\
\hline $\mathrm{H}$ & 4.468978 & -2.538748 & 0.963184 \\
\hline $\mathrm{H}$ & 4.142781 & -3.823595 & -0.162374 \\
\hline $\mathrm{Zn}$ & 1.99973 & -3.020638 & 1.038079 \\
\hline C & 4.424665 & -4.510389 & 1.861818 \\
\hline $\mathrm{H}$ & 3.966193 & -5.497531 & 1.704485 \\
\hline $\mathrm{H}$ & 5.514681 & -4.650872 & 1.796713 \\
\hline $\mathrm{H}$ & 4.203687 & -4.221876 & 2.897521 \\
\hline $\mathrm{Zn}$ & 1.91735 & -4.604496 & -1.373481 \\
\hline C & 2.999199 & -6.045168 & -2.08404 \\
\hline $\mathrm{H}$ & 3.053842 & -5.941939 & -3.177493 \\
\hline $\mathrm{H}$ & 4.019999 & -5.867855 & -1.714436 \\
\hline$C$ & 2.53251 & -7.445658 & -1.705049 \\
\hline $\mathrm{H}$ & 1.523433 & -7.6621 & -2.077352 \\
\hline $\mathrm{H}$ & 3.196126 & -8.221294 & -2.114046 \\
\hline $\mathrm{H}$ & 2.509134 & -7.590494 & -0.617649 \\
\hline
\end{tabular}


Table S16. Structure for a ZnEt group added to two HB sites on the node that are opposite of each other.

$E=-5470.67999544$ Hartrees, $G=-5469.928857$ Hartrees

\begin{tabular}{|c|c|c|c|}
\hline Atom & $X$ & $\mathrm{Y}$ & Z \\
\hline $\mathrm{C}$ & 6.202436 & 1.174286 & 2.564808 \\
\hline$C$ & -4.762681 & 4.175104 & -2.511157 \\
\hline$C$ & 6.199271 & 1.160174 & -2.573415 \\
\hline$C$ & -4.717666 & 4.227239 & 2.451908 \\
\hline$C$ & 4.975433 & 1.765404 & 2.900505 \\
\hline C & -3.391605 & 4.095169 & -2.797449 \\
\hline C & 4.976438 & 1.767608 & -2.896069 \\
\hline C & -3.354092 & 4.14376 & 2.770848 \\
\hline$C$ & 4.968568 & 2.88209 & 3.749913 \\
\hline C & -2.831477 & 5.003301 & -3.708136 \\
\hline $\mathrm{C}$ & 4.97051 & 2.841489 & -3.798742 \\
\hline C & -2.807059 & 5.072911 & 3.6677 \\
\hline C & 6.163613 & 3.40488 & 4.238658 \\
\hline C & -3.619664 & 5.988945 & -4.294451 \\
\hline C & 6.161608 & 3.318184 & -4.339312 \\
\hline $\mathrm{C}$ & -3.599402 & 6.081135 & 4.209704 \\
\hline $\mathrm{C}$ & 7.400946 & 2.820971 & 3.903182 \\
\hline $\mathrm{C}$ & -4.989961 & 6.099818 & -3.987761 \\
\hline $\mathrm{C}$ & 7.39736 & 2.732607 & -4.000444 \\
\hline C & -4.964246 & 6.187946 & 3.876781 \\
\hline $\mathrm{C}$ & 7.394195 & 1.691436 & 3.062347 \\
\hline $\mathrm{C}$ & -5.548456 & 5.160035 & -3.101232 \\
\hline $\mathrm{C}$ & 7.387902 & 1.63279 & -3.121054 \\
\hline $\mathrm{C}$ & -5.509961 & 5.23015 & 3.001264 \\
\hline $\mathrm{C}$ & -6.203696 & -1.293319 & -2.459201 \\
\hline $\mathrm{C}$ & 4.759237 & -4.155211 & 2.610114 \\
\hline $\mathrm{C}$ & -6.231343 & -1.226308 & 2.512887 \\
\hline C & 4.772294 & -4.171088 & -2.552185 \\
\hline $\mathrm{C}$ & -4.985061 & -1.911995 & -2.775316 \\
\hline C & 3.395703 & -4.068059 & 2.929736 \\
\hline C & -5.008949 & -1.852574 & 2.798952 \\
\hline $\mathrm{C}$ & 3.414237 & -4.067003 & -2.891074 \\
\hline $\mathrm{C}$ & -4.979694 & -2.990577 & -3.671476 \\
\hline $\mathrm{C}$ & 2.851617 & -4.99126 & 3.835883 \\
\hline $\mathrm{C}$ & -4.985195 & -2.917986 & 3.710917 \\
\hline C & 2.852604 & -5.028231 & -3.745278 \\
\hline $\mathrm{C}$ & -6.17115 & -3.460783 & -4.215274 \\
\hline $\mathrm{C}$ & 3.643961 & -5.999429 & 4.378178 \\
\hline
\end{tabular}




\begin{tabular}{|c|c|c|c|}
\hline$C$ & -6.163752 & -3.36779 & 4.29836 \\
\hline$C$ & 3.626399 & -6.079269 & -4.232076 \\
\hline$C$ & -7.403057 & -2.863784 & -3.883876 \\
\hline$C$ & 5.007125 & -6.114007 & 4.040631 \\
\hline$C$ & -7.401178 & -2.76917 & 3.991216 \\
\hline$C$ & 4.987984 & -6.197636 & -3.890427 \\
\hline$C$ & -7.392729 & -1.759923 & -3.010431 \\
\hline$C$ & 5.551689 & -5.157825 & 3.16126 \\
\hline$C$ & -7.407897 & -1.677283 & 3.103219 \\
\hline$C$ & 5.545912 & -5.216391 & -3.048324 \\
\hline$C$ & 3.721152 & 1.282056 & -2.251348 \\
\hline$C$ & 3.712474 & 1.249343 & 2.302962 \\
\hline$C$ & 2.553327 & -3.02172 & 2.276706 \\
\hline C & -3.753651 & -1.408094 & 2.13273 \\
\hline C & -3.723442 & -1.419378 & -2.151147 \\
\hline$C$ & -2.53441 & 3.076782 & -2.131259 \\
\hline$C$ & 2.576502 & -2.991253 & -2.288433 \\
\hline C & -2.514152 & 3.076803 & 2.152287 \\
\hline $\mathrm{H}$ & 6.203093 & 0.31452 & 1.898365 \\
\hline $\mathrm{H}$ & -5.192866 & 3.460943 & -1.81229 \\
\hline $\mathrm{H}$ & 6.200536 & 0.325204 & -1.876371 \\
\hline $\mathrm{H}$ & -5.137864 & 3.497124 & 1.763777 \\
\hline $\mathrm{H}$ & 4.015962 & 3.349137 & 3.993554 \\
\hline $\mathrm{H}$ & -1.772427 & 4.925649 & -3.944295 \\
\hline $\mathrm{H}$ & 4.021413 & 3.312388 & -4.04809 \\
\hline $\mathrm{H}$ & -1.754598 & 4.991962 & 3.932323 \\
\hline $\mathrm{H}$ & 6.138504 & 4.281234 & 4.884883 \\
\hline $\mathrm{H}$ & -3.166442 & 6.690592 & -4.993528 \\
\hline $\mathrm{H}$ & 6.135518 & 4.166074 & -5.022406 \\
\hline $\mathrm{H}$ & -3.155267 & 6.797822 & 4.899222 \\
\hline $\mathrm{H}$ & 8.337183 & 1.219749 & 2.788604 \\
\hline $\mathrm{H}$ & -6.609509 & 5.211662 & -2.860621 \\
\hline $\mathrm{H}$ & 8.327635 & 1.151949 & -2.852137 \\
\hline $\mathrm{H}$ & -6.565993 & 5.281587 & 2.738966 \\
\hline $\mathrm{H}$ & -6.199176 & -0.454886 & -1.766127 \\
\hline $\mathrm{H}$ & 5.179413 & -3.432469 & 1.914534 \\
\hline $\mathrm{H}$ & -6.241995 & -0.396229 & 1.809558 \\
\hline $\mathrm{H}$ & 5.202495 & -3.42688 & -1.88577 \\
\hline $\mathrm{H}$ & -4.029632 & -3.454959 & -3.928056 \\
\hline $\mathrm{H}$ & 1.79534 & -4.921801 & 4.089285 \\
\hline $\mathrm{H}$ & -4.032842 & -3.397084 & 3.928975 \\
\hline $\mathrm{H}$ & 1.797761 & -4.951258 & -4.000519 \\
\hline $\mathrm{H}$ & -6.149647 & -4.307793 & -4.899778 \\
\hline
\end{tabular}




$\begin{array}{rrrr}\mathrm{H} & 3.197828 & -6.715683 & 5.066795 \\ \mathrm{H} & -6.128788 & -4.205178 & 4.994073 \\ \mathrm{H} & 3.168189 & -6.822335 & -4.883446 \\ \mathrm{H} & -8.331381 & -1.273052 & -2.748475 \\ \mathrm{H} & 6.606617 & -5.211365 & 2.895244 \\ \mathrm{H} & -8.349651 & -1.186487 & 2.860621 \\ \mathrm{H} & 6.597674 & -5.280227 & -2.772032 \\ \mathrm{H} & 8.328646 & 3.110244 & -4.41587 \\ \mathrm{H} & -5.602814 & 6.876378 & -4.439396 \\ \mathrm{H} & 5.622951 & -6.906221 & 4.459547 \\ \mathrm{H} & 5.590337 & -7.020371 & -4.268274 \\ \mathrm{H} & -8.323253 & -3.129023 & 4.441949 \\ \mathrm{H} & -5.581929 & 6.977784 & 4.297896 \\ \mathrm{H} & -8.334645 & -3.23581 & -4.304036 \\ \mathrm{H} & 8.3341 & 3.231145 & 4.282204 \\ \mathrm{H} & -1.907486 & 0.499131 & -2.806782 \\ \mathrm{H} & 2.854024 & 1.918709 & 0.001814 \\ \mathrm{H} & 0.49353 & -1.800964 & 4.33471 \\ \mathrm{H} & -0.033052 & -0.30736 & 4.111829 \\ \mathrm{H} & 0.324032 & -1.846695 & -4.359627 \\ \mathrm{H} & -1.479819 & -4.200963 & -2.149002 \\ \mathrm{H} & -1.921918 & 0.465822 & 2.854759 \\ \mathrm{H} & 1.114299 & -3.294154 & -0.109324 \\ \mathrm{H} & 1.129646 & 1.458309 & 4.438631 \\ \mathrm{H} & 0.106539 & 0.248925 & -4.046167 \\ \mathrm{H} & 1.322413 & 1.257276 & -4.258909 \\ \mathrm{H} & 0.257436 & 4.322854 & 1.73664 \\ \mathrm{O} & 1.020272 & -0.325266 & 1.495978 \\ \mathrm{O} & 3.116216 & -2.312214 & 1.390508 \\ \mathrm{O} & 3.13497 & -2.262323 & -1.40807 \\ \mathrm{O} & 3.827453 & 0.346663 & 1.411981 \\ \mathrm{O} & 2.624515 & 1.767345 & 2.692582 \\ \mathrm{O} & 1.063891 & 3.791241 & 1.661214 \\ \mathrm{O} & -1.282047 & 3.065497 & 2.44027 \\ \mathrm{O} & -1.290389 & 3.075581 & -2.401105 \\ \mathrm{O} & -3.104709 & 2.254195 & 1.382804 \\ \mathrm{O} & -3.110818 & 2.275936 & -1.332392 \\ \mathrm{O} & -3.853813 & -0.427215 & 1.32745 \\ \mathrm{O} & -3.821793 & -0.412809 & -1.377404 \\ \mathrm{O} & -2.648357 & -2.021098 & -2.444665 \\ \mathrm{O} & -2.675625 & -2.01143 & 2.425108 \\ \mathrm{O} & -1.468694 & -1.263449 & -0.003905 \\ & -1.34271 & 0.317198 & 2.093025\end{array}$




$\begin{array}{lrrr}\mathrm{O} & 0.310923 & 1.240519 & 3.96992 \\ \mathrm{O} & -0.277477 & -1.300857 & 4.02152 \\ \mathrm{O} & 0.397031 & 1.227301 & -3.964084 \\ \mathrm{O} & -0.602288 & 1.673648 & 0.051923 \\ \mathrm{O} & 1.919379 & 1.656449 & 0.050491 \\ \mathrm{O} & 2.620589 & 1.806307 & -2.61532 \\ \mathrm{O} & 1.055556 & 3.818339 & -1.454259 \\ \mathrm{O} & 3.847857 & 0.400028 & -1.343944 \\ \mathrm{O} & -1.053551 & -3.816009 & 1.534601 \\ \mathrm{O} & -0.993973 & -3.823969 & -1.398586 \\ \mathrm{O} & 0.802366 & -2.382661 & -0.025111 \\ \mathrm{O} & 1.334689 & -2.935864 & 2.635027 \\ \mathrm{O} & 1.37129 & -2.900229 & -2.667199 \\ \mathrm{O} & -0.347147 & -1.298909 & -3.927347 \\ \mathrm{O} & -1.321252 & 0.366162 & -2.04755 \\ \mathrm{O} & 1.036061 & -0.271238 & -1.468165 \\ \mathrm{Zr} & -2.407101 & 0.621315 & 0.012378 \\ \mathrm{Zr} & 0.483111 & 1.70036 & 1.920288 \\ \mathrm{Zr} & -0.475361 & -1.767383 & 1.782159 \\ \mathrm{Zr} & -0.439074 & -1.74316 & -1.866162 \\ \mathrm{Zr} & 2.405558 & -0.694042 & 0.010142 \\ \mathrm{Zr} & 0.467052 & 1.755424 & -1.720423 \\ \mathrm{Zn} & -2.436485 & -3.647233 & 0.05221 \\ \mathrm{Zn} & 2.275751 & 4.118856 & 0.141191 \\ \mathrm{H} & -0.341143 & -4.407401 & 1.254033 \\ \mathrm{H} & 0.279864 & 4.384572 & -1.580131 \\ \mathrm{C} & -4.374275 & -3.936024 & 0.045898 \\ \mathrm{H} & -4.859187 & -2.98152 & -0.212213 \\ \mathrm{H} & -4.688071 & -4.14698 & 1.078308 \\ \mathrm{C} & 4.228798 & 4.246042 & -0.041085 \\ \mathrm{H} & 4.68659 & 4.533592 & 0.915408 \\ \mathrm{H} & 4.591743 & 3.215919 & -0.222418 \\ \mathrm{H} & 4.722237 & 5.150387 & -1.164332 \\ \mathrm{H} & 5.803384 & 5.045185 & -1.345638 \\ \mathrm{H} & 4.219193 & 4.933385 & -2.115991 \\ \mathrm{H} & 4.534336 & 6.209167 & -0.94135 \\ \mathrm{H} & -4.869373 & -5.042471 & -0.878823 \\ \mathrm{H} & & -5.034029 & -0.534322 \\ \mathrm{H} & -595454 & -5.070291 & -0.949491 \\ \mathrm{H} & -4.92875 & -1.907106\end{array}$


Table S17. Structure for $2 \mathrm{H}_{2} \mathrm{O}-2 \mathrm{Et}-\mathrm{O}$.

$E=-5470.64989224$ Hartrees, $G=-5469.900509$ Hartrees

\begin{tabular}{|c|c|c|c|}
\hline Atom & $x$ & $Y$ & Z \\
\hline C & -5.901937 & 2.787215 & -2.47627 \\
\hline$C$ & 5.507136 & 2.784253 & 2.507398 \\
\hline$C$ & -5.860573 & 2.685481 & 2.660793 \\
\hline$C$ & 5.436512 & 2.930633 & -2.453294 \\
\hline$C$ & -4.567014 & 3.048879 & -2.818574 \\
\hline $\mathrm{C}$ & 4.163966 & 3.054288 & 2.809314 \\
\hline$C$ & -4.520108 & 2.952908 & 2.977062 \\
\hline$C$ & 4.095085 & 3.204734 & -2.757872 \\
\hline C & -4.2803 & 4.140665 & -3.65184 \\
\hline$C$ & 3.863504 & 4.060269 & 3.739663 \\
\hline C & -4.231004 & 3.973725 & 3.894906 \\
\hline C & 3.797398 & 4.259195 & -3.633201 \\
\hline$C$ & -5.304781 & 4.961079 & -4.118389 \\
\hline C & 4.883299 & 4.800278 & 4.330339 \\
\hline$C$ & -5.255118 & 4.731119 & 4.456794 \\
\hline C & 4.818018 & 5.038958 & -4.170685 \\
\hline C & -6.647842 & 4.708967 & -3.776169 \\
\hline$C$ & 6.233509 & 4.560655 & 4.008507 \\
\hline C & -6.602584 & 4.488435 & 4.124742 \\
\hline $\mathrm{C}$ & 6.167135 & 4.785978 & -3.853107 \\
\hline C & -6.924765 & 3.601481 & -2.951739 \\
\hline C & 6.524448 & 3.524145 & 3.101837 \\
\hline C & -6.883293 & 3.438185 & 3.229652 \\
\hline$C$ & 6.455465 & 3.705424 & -2.998089 \\
\hline C & 5.493793 & -2.869155 & 2.359486 \\
\hline$C$ & -5.87741 & -2.732594 & -2.615585 \\
\hline$C$ & 5.497154 & -2.727065 & -2.611056 \\
\hline$C$ & -5.851976 & -2.832286 & 2.545742 \\
\hline C & 4.159713 & -3.159278 & 2.681695 \\
\hline$C$ & -4.539917 & -2.993355 & -2.950437 \\
\hline$C$ & 4.152656 & -3.013156 & -2.89117 \\
\hline$C$ & -4.510069 & -3.086278 & 2.869434 \\
\hline C & 3.884625 & -4.215399 & 3.562267 \\
\hline$C$ & -4.258821 & -4.009633 & -3.876479 \\
\hline $\mathrm{C}$ & 3.848342 & -4.021279 & -3.817853 \\
\hline$C$ & -4.207401 & -4.173877 & 3.702847 \\
\hline$C$ & 4.919599 & -4.985024 & 4.084646 \\
\hline C & -5.288061 & -4.771128 & -4.423372 \\
\hline C & 4.86687 & -4.748682 & -4.426047 \\
\hline
\end{tabular}




\begin{tabular}{|c|c|c|c|}
\hline C & -5.221338 & -4.99901 & 4.183995 \\
\hline C & 6.260815 & -4.718932 & 3.746762 \\
\hline C & -6.632079 & -4.537441 & -4.070824 \\
\hline C & 6.219047 & -4.493289 & -4.125552 \\
\hline C & -6.570348 & -4.757856 & 3.857394 \\
\hline C & 6.527418 & -3.634785 & 2.889401 \\
\hline C & -6.9054 & -3.48854 & -3.171256 \\
\hline C & 6.51342 & -3.455008 & -3.222154 \\
\hline C & -6.864188 & -3.652073 & 3.036317 \\
\hline C & -3.437163 & 2.171989 & 2.310295 \\
\hline C & -3.474347 & 2.215762 & -2.243889 \\
\hline C & -3.451746 & -2.209439 & -2.29316 \\
\hline C & 3.059664 & -2.273605 & -2.203754 \\
\hline C & 3.06198 & -2.348508 & 2.080134 \\
\hline C & 3.068411 & 2.301486 & 2.139421 \\
\hline C & -3.42908 & -2.2517 & 2.272019 \\
\hline C & 3.013702 & 2.380272 & -2.142788 \\
\hline $\mathrm{H}$ & -6.11774 & 1.943467 & -1.825025 \\
\hline $\mathrm{H}$ & 5.733461 & 1.998399 & 1.79014 \\
\hline $\mathrm{H}$ & -6.08121 & 1.888272 & 1.95511 \\
\hline $\mathrm{H}$ & 5.661098 & 2.108473 & -1.77759 \\
\hline $\mathrm{H}$ & -3.243933 & 4.34501 & -3.912878 \\
\hline $\mathrm{H}$ & 2.822307 & 4.253617 & 3.986392 \\
\hline $\mathrm{H}$ & -3.191459 & 4.180405 & 4.14061 \\
\hline $\mathrm{H}$ & 2.754527 & 4.465525 & -3.866423 \\
\hline $\mathrm{H}$ & -5.060996 & 5.809704 & -4.75573 \\
\hline $\mathrm{H}$ & 4.631028 & 5.585466 & 5.041666 \\
\hline $\mathrm{H}$ & -5.007432 & 5.529115 & 5.15513 \\
\hline $\mathrm{H}$ & 4.566548 & 5.860836 & -4.839427 \\
\hline $\mathrm{H}$ & -7.955013 & 3.381973 & -2.67495 \\
\hline $\mathrm{H}$ & 7.560846 & 3.306255 & 2.848038 \\
\hline $\mathrm{H}$ & -7.917165 & 3.218456 & 2.966867 \\
\hline $\mathrm{H}$ & 7.490944 & 3.481247 & -2.745321 \\
\hline $\mathrm{H}$ & 5.700294 & -2.044486 & 1.681165 \\
\hline $\mathrm{H}$ & -6.091471 & -1.938164 & -1.904766 \\
\hline $\mathrm{H}$ & 5.725635 & -1.93917 & -1.896773 \\
\hline $\mathrm{H}$ & -6.081681 & -1.991275 & 1.895723 \\
\hline $\mathrm{H}$ & 2.846988 & -4.429974 & 3.811379 \\
\hline $\mathrm{H}$ & -3.222061 & -4.210697 & -4.138141 \\
\hline $\mathrm{H}$ & 2.804794 & -4.230593 & -4.041897 \\
\hline $\mathrm{H}$ & -3.166792 & -4.372811 & 3.950775 \\
\hline $\mathrm{H}$ & 4.686055 & -5.810376 & 4.755609 \\
\hline $\mathrm{H}$ & -5.046822 & -5.566631 & -5.126888 \\
\hline
\end{tabular}




$\begin{array}{rrrr}\mathrm{H} & 4.612933 & -5.535252 & -5.135218 \\ \mathrm{H} & -4.963906 & -5.844619 & 4.820046 \\ \mathrm{H} & 7.557276 & -3.400549 & 2.623182 \\ \mathrm{H} & -7.936333 & -3.273721 & -2.89335 \\ \mathrm{H} & 7.551311 & -3.226105 & -2.984797 \\ \mathrm{H} & -7.899049 & -3.438653 & 2.772237 \\ \mathrm{H} & -7.401964 & 5.08434 & 4.558237 \\ \mathrm{H} & 7.028769 & 5.146748 & 4.462697 \\ \mathrm{H} & -7.434102 & -5.137595 & -4.493356 \\ \mathrm{H} & -7.360734 & -5.404247 & 4.230954 \\ \mathrm{H} & 7.013541 & -5.069715 & -4.593324 \\ \mathrm{H} & 6.963597 & 5.398509 & -4.26896 \\ \mathrm{H} & 7.068969 & -5.324568 & 4.149977 \\ \mathrm{H} & -7.447178 & 5.350461 & -4.139096 \\ \mathrm{H} & 1.811783 & 0.012986 & 2.791968 \\ \mathrm{H} & -2.157147 & 2.841619 & 0.100358 \\ \mathrm{H} & -1.182917 & -1.55398 & -4.273881 \\ \mathrm{H} & -0.250373 & -0.275825 & -4.101759 \\ \mathrm{H} & -0.86986 & -1.753486 & 4.422273 \\ \mathrm{H} & -1.006232 & -4.310902 & 1.237972 \\ \mathrm{H} & 0.246577 & -4.402441 & -2.133156 \\ \mathrm{H} & 1.774323 & 0.000782 & -2.848164 \\ \mathrm{H} & -2.133867 & -2.866619 & -0.099923 \\ \mathrm{H} & -0.933323 & 1.74111 & -4.44714 \\ \mathrm{H} & -0.199999 & 0.266863 & 4.07035 \\ \mathrm{H} & -1.139484 & 1.537413 & 4.25762 \\ \mathrm{H} & 0.252836 & 4.408836 & 2.075056 \\ \mathrm{H} & -1.082528 & 4.285992 & -1.277896 \\ \mathrm{O} & -1.263318 & -0.008472 & -1.482864 \\ \mathrm{O} & -3.805478 & -1.406676 & -1.375143 \\ \mathrm{O} & -3.784774 & -1.375996 & 1.424619 \\ \mathrm{O} & -3.812944 & 1.337372 & -1.392101 \\ \mathrm{O} & -2.292234 & 2.463134 & -2.629996 \\ \mathrm{O} & -0.234939 & 3.940942 & -1.591695 \\ \mathrm{O} & 1.814255 & 2.679852 & -2.414277 \\ \mathrm{O} & 1.868988 & 2.600555 & 2.440867 \\ \mathrm{O} & 3.385061 & 1.41927 & -1.393375 \\ \mathrm{O} & 3.416232 & 1.39733 & 1.31669 \\ \mathrm{O} & 3.411365 & -1.367281 & -1.385009 \\ \mathrm{O} & 3.413747 & -1.385716 & 1.323342 \\ \mathrm{O} & 1.869984 & -2.660797 & 2.367959 \\ \mathrm{O} & 1.859267 & -2.582855 & -2.489166 \\ \mathrm{O} & 0.89919 & -1.554675 & 0.002632 \\ & & & \end{array}$




\begin{tabular}{lrrr}
$\mathrm{O}$ & 1.176239 & -0.001416 & -2.086388 \\
$\mathrm{O}$ & -0.21225 & 1.315534 & -3.962105 \\
$\mathrm{O}$ & -0.282527 & -1.297271 & -4.012531 \\
$\mathrm{O}$ & -0.240809 & 1.288047 & 3.983826 \\
$\mathrm{O}$ & 0.883715 & 1.55732 & -0.046519 \\
$\mathrm{O}$ & -1.594001 & 2.062821 & -0.009167 \\
$\mathrm{O}$ & -2.235973 & 2.390961 & 2.668087 \\
$\mathrm{O}$ & -0.196371 & 3.935796 & 1.356712 \\
$\mathrm{O}$ & -3.797501 & 1.360336 & 1.403505 \\
$\mathrm{O}$ & -0.197763 & -3.937148 & -1.406774 \\
$\mathrm{O}$ & -0.15951 & -3.952351 & 1.539122 \\
$\mathrm{O}$ & -1.576071 & -2.082904 & 0.001649 \\
$\mathrm{O}$ & -2.255139 & -2.413314 & -2.67218 \\
$\mathrm{O}$ & -2.237431 & -2.498599 & 2.630915 \\
$\mathrm{O}$ & -0.153428 & -1.325864 & 3.932133 \\
$\mathrm{O}$ & 1.201809 & 0.009448 & 2.039662 \\
$\mathrm{O}$ & -1.239754 & -0.008932 & 1.468505 \\
$\mathrm{Zr}$ & 2.309123 & 0.009514 & -0.029201 \\
$\mathrm{Zr}$ & -0.241164 & 1.793786 & -1.91455 \\
$\mathrm{Zr}$ & -0.186298 & -1.798651 & -1.761154 \\
$\mathrm{Zr}$ & -0.19904 & -1.805043 & 1.885209 \\
$\mathrm{Zr}$ & -2.699117 & -0.016876 & 0.005665 \\
$\mathrm{Zr}$ & -0.176309 & 1.796553 & 1.731674 \\
$\mathrm{C}$ & 3.008007 & -4.896589 & -0.213546 \\
$\mathrm{H}$ & 3.725406 & -4.079872 & -0.032056 \\
$\mathrm{H}$ & 3.086052 & -5.108668 & -1.290231 \\
$\mathrm{Zn}$ & 1.220867 & -4.175445 & 0.130566 \\
$\mathrm{C}$ & 3.41088 & -6.129642 & 0.585845 \\
$\mathrm{H}$ & 2.791169 & -7.000396 & 0.333034 \\
$\mathrm{H}$ & 4.458123 & -6.418376 & 0.40451 \\
$\mathrm{H}$ & 3.311743 & -5.974387 & 1.667119 \\
$\mathrm{Zn}$ & 1.175379 & 4.190869 & -0.220699 \\
$\mathrm{C}$ & 2.944481 & 4.985624 & 0.038063 \\
$\mathrm{H}$ & 3.29873 & 5.38502 & -0.922461 \\
$\mathrm{H}$ & 3.645661 & 4.173219 & 0.285609 \\
$\mathrm{C}$ & 3.001551 & 6.072317 & 1.104695 \\
$\mathrm{H}$ & 4.030132 & 6.408673 & 1.309644 \\
$\mathrm{H}$ & 2.425265 & 6.960447 & 0.811947 \\
$\mathrm{H}$ & 2.594163 & 5.734494 & 2.068002 \\
& & & \\
\hline
\end{tabular}


Table S18. Structure for a ZnEt group added to two $\mu \mathrm{OH}$ sites on the node that are opposite of each other.

$E=-5470.66906385$ Hartrees, $G=-5469.91957$ Hartrees

\begin{tabular}{lrrr}
\hline Atom & $\mathrm{X}$ & \multicolumn{1}{c}{$\mathrm{Y}$} & \multicolumn{1}{c}{$\mathrm{Z}$} \\
\hline \hline $\mathrm{C}$ & -5.390359 & 2.853949 & -2.50995 \\
$\mathrm{C}$ & 6.005325 & 2.696581 & 2.501797 \\
$\mathrm{C}$ & -5.362383 & 2.828126 & 2.628145 \\
$\mathrm{C}$ & 5.948618 & 2.770128 & -2.460858 \\
$\mathrm{C}$ & -4.049693 & 3.083665 & -2.852744 \\
$\mathrm{C}$ & 4.667186 & 2.997972 & 2.796299 \\
$\mathrm{C}$ & -4.017519 & 3.07328 & 2.943789 \\
$\mathrm{C}$ & 4.613341 & 3.067276 & -2.771766 \\
$\mathrm{C}$ & -3.739266 & 4.156904 & -3.701459 \\
$\mathrm{C}$ & 4.384945 & 4.023558 & 3.710812 \\
$\mathrm{C}$ & -3.709991 & 4.101704 & 3.847035 \\
$\mathrm{C}$ & 4.339322 & 4.113526 & -3.664672 \\
$\mathrm{C}$ & -4.746025 & 4.990645 & -4.182727 \\
$\mathrm{C}$ & 5.418095 & 4.751699 & 4.29297 \\
$\mathrm{C}$ & -4.719918 & 4.887784 & 4.395025 \\
$\mathrm{C}$ & 5.376376 & 4.864723 & -4.210852 \\
$\mathrm{C}$ & -6.094633 & 4.770655 & -3.840164 \\
$\mathrm{C}$ & 6.763925 & 4.480266 & 3.978121 \\
$\mathrm{C}$ & -6.071252 & 4.667246 & 4.063246 \\
$\mathrm{C}$ & 6.719433 & 4.589479 & -3.886072 \\
$\mathrm{C}$ & -6.395567 & 3.681381 & -3.000035 \\
$\mathrm{C}$ & 7.03598 & 3.424707 & 3.087712 \\
$\mathrm{C}$ & -6.371029 & 3.60959 & 3.183181 \\
$\mathrm{C}$ & 6.984117 & 3.516243 & -3.014398 \\
$\mathrm{C}$ & 5.878727 & -2.957005 & 2.438009 \\
$\mathrm{C}$ & -5.476428 & -2.666709 & -2.567024 \\
$\mathrm{C}$ & 5.895914 & -2.889249 & -2.53406 \\
$\mathrm{C}$ & -5.464394 & -2.68981 & 2.595261 \\
$\mathrm{C}$ & 4.538376 & -3.21547 & 2.761158 \\
$\mathrm{C}$ & -4.14371 & -2.95918 & -2.894639 \\
$\mathrm{C}$ & 4.546558 & -3.15242 & -2.813312 \\
$\mathrm{C}$ & -4.128577 & -2.965824 & 2.926035 \\
$\mathrm{C}$ & -3.240184 & -4.252605 & 3.656644 \\
$\mathrm{C}$ & 4.224099 & -4.167995 & -3.725601 \\
$\mathrm{C}$ & 5.258334 & -5.034938 & 4.193022
\end{tabular}




\begin{tabular}{|c|c|c|c|}
\hline$C$ & -4.924166 & -4.743408 & -4.342797 \\
\hline$C$ & 5.229148 & -4.924678 & -4.320351 \\
\hline$C$ & -4.881034 & -4.844052 & 4.267149 \\
\hline$C$ & 6.605367 & -4.800874 & 3.854576 \\
\hline$C$ & -6.263993 & -4.477575 & -3.997137 \\
\hline$C$ & 6.585516 & -4.691994 & -4.020306 \\
\hline$C$ & -6.224201 & -4.580793 & 3.933613 \\
\hline$C$ & 6.895588 & -3.735214 & 2.981848 \\
\hline$C$ & -6.518168 & -3.41009 & -3.113966 \\
\hline$C$ & 6.898695 & -3.646458 & -3.131719 \\
\hline$C$ & -6.493953 & -3.481722 & 3.095438 \\
\hline$C$ & -2.94901 & 2.260948 & 2.291433 \\
\hline$C$ & -2.975243 & 2.237497 & -2.262975 \\
\hline$C$ & -3.041447 & -2.187543 & -2.246326 \\
\hline$C$ & 3.467158 & -2.380941 & -2.139626 \\
\hline$C$ & 3.458496 & -2.39192 & 2.144863 \\
\hline$C$ & 3.558211 & 2.257382 & 2.135008 \\
\hline$C$ & -3.029727 & -2.162083 & 2.319003 \\
\hline$C$ & 3.51459 & 2.272849 & -2.148653 \\
\hline $\mathrm{H}$ & -5.623357 & 2.0264 & -1.843176 \\
\hline $\mathrm{H}$ & 6.217627 & 1.892678 & 1.800556 \\
\hline $\mathrm{H}$ & -5.5964 & 2.02735 & 1.930145 \\
\hline $\mathrm{H}$ & 6.154744 & 1.950952 & -1.776052 \\
\hline $\mathrm{H}$ & -2.697218 & 4.340238 & -3.955743 \\
\hline $\mathrm{H}$ & 3.346312 & 4.240563 & 3.949832 \\
\hline $\mathrm{H}$ & -2.666496 & 4.293645 & 4.090066 \\
\hline $\mathrm{H}$ & 3.304953 & 4.325094 & -3.928419 \\
\hline $\mathrm{H}$ & -4.483345 & 5.827918 & -4.828058 \\
\hline $\mathrm{H}$ & 5.180972 & 5.549787 & 4.995512 \\
\hline $\mathrm{H}$ & -4.45786 & 5.692072 & 5.081173 \\
\hline $\mathrm{H}$ & 5.144163 & 5.677012 & -4.898379 \\
\hline $\mathrm{H}$ & -7.43058 & 3.488676 & -2.720054 \\
\hline $\mathrm{H}$ & 8.068801 & 3.182307 & 2.840484 \\
\hline $\mathrm{H}$ & -7.408499 & 3.4086 & 2.918866 \\
\hline $\mathrm{H}$ & 8.014716 & 3.27337 & -2.758349 \\
\hline $\mathrm{H}$ & 6.102837 & -2.144043 & 1.751566 \\
\hline $\mathrm{H}$ & -5.675104 & -1.85992 & -1.86466 \\
\hline $\mathrm{H}$ & 6.139018 & -2.092233 & -1.835003 \\
\hline $\mathrm{H}$ & -5.674459 & -1.858023 & 1.926048 \\
\hline $\mathrm{H}$ & 3.202011 & -4.43247 & 3.928796 \\
\hline $\mathrm{H}$ & -2.847214 & -4.223351 & -4.057324 \\
\hline $\mathrm{H}$ & 3.177017 & -4.354185 & -3.95341 \\
\hline $\mathrm{H}$ & -2.812723 & -4.267916 & 4.020872 \\
\hline
\end{tabular}




\begin{tabular}{|c|c|c|c|}
\hline $\mathrm{H}$ & 5.00836 & -5.840329 & 4.882445 \\
\hline $\mathrm{H}$ & -4.69728 & -5.555504 & -5.03232 \\
\hline $\mathrm{H}$ & 4.961549 & -5.714587 & -5.02115 \\
\hline $\mathrm{H}$ & -4.641985 & -5.687719 & 4.913326 \\
\hline $\mathrm{H}$ & 7.930988 & -3.524396 & 2.716903 \\
\hline $\mathrm{H}$ & -7.545096 & -3.173348 & -2.838452 \\
\hline $\mathrm{H}$ & 7.940898 & -3.434337 & -2.896413 \\
\hline $\mathrm{H}$ & -7.523576 & -3.254801 & 2.821459 \\
\hline $\mathrm{H}$ & -6.859478 & 5.28621 & 4.485157 \\
\hline $\mathrm{H}$ & 7.570066 & 5.056148 & 4.426714 \\
\hline $\mathrm{H}$ & -7.077017 & -5.06886 & -4.411493 \\
\hline $\mathrm{H}$ & -7.028271 & -5.20697 & 4.312998 \\
\hline $\mathrm{H}$ & 7.369564 & -5.290284 & -4.478709 \\
\hline $\mathrm{H}$ & 7.528809 & 5.178809 & -4.310708 \\
\hline $\mathrm{H}$ & 7.400325 & -5.415315 & 4.270845 \\
\hline $\mathrm{H}$ & -6.880101 & 5.423655 & -4.213442 \\
\hline $\mathrm{H}$ & 2.215633 & -0.006098 & 2.854064 \\
\hline $\mathrm{H}$ & -0.760459 & -1.59886 & -4.177984 \\
\hline $\mathrm{H}$ & 0.19862 & -0.350198 & -4.011932 \\
\hline $\mathrm{H}$ & -0.544973 & -1.66504 & 4.364401 \\
\hline $\mathrm{H}$ & 0.431272 & -4.145659 & 0.440013 \\
\hline $\mathrm{H}$ & 0.424992 & -4.661652 & -1.766169 \\
\hline $\mathrm{H}$ & 1.166725 & -4.296768 & 1.832204 \\
\hline $\mathrm{H}$ & 2.192772 & -0.075503 & -2.847668 \\
\hline $\mathrm{H}$ & -0.528068 & 1.660016 & -4.335878 \\
\hline $\mathrm{H}$ & 0.242012 & 0.319681 & 4.033487 \\
\hline $\mathrm{H}$ & -0.676241 & 1.597108 & 4.210897 \\
\hline $\mathrm{H}$ & 1.272886 & 4.236638 & -1.832253 \\
\hline $\mathrm{H}$ & 0.501303 & 4.647838 & 1.781553 \\
\hline $\mathrm{H}$ & 0.549472 & 4.117635 & -0.43174 \\
\hline $\mathrm{O}$ & -0.817205 & -0.056547 & -1.507898 \\
\hline O & -3.375188 & -1.350774 & -1.356033 \\
\hline O & -3.361644 & -1.28212 & 1.466983 \\
\hline O & -3.328704 & 1.359777 & -1.41732 \\
\hline O & -1.778055 & 2.478499 & -2.624803 \\
\hline O & 0.411711 & 4.031475 & -1.424903 \\
\hline O & 2.32006 & 2.599489 & -2.419897 \\
\hline $\mathrm{O}$ & 2.36442 & 2.614668 & 2.385695 \\
\hline 0 & 3.875925 & 1.312461 & -1.399931 \\
\hline 0 & 3.90572 & 1.32138 & 1.348101 \\
\hline O & 3.849737 & -1.459197 & -1.352415 \\
\hline $\mathrm{O}$ & 3.841958 & -1.444297 & 1.39144 \\
\hline O & 2.257389 & -2.685576 & 2.424128 \\
\hline
\end{tabular}




$\begin{array}{lrrr}\mathrm{O} & 2.262092 & -2.701197 & -2.383085 \\ \mathrm{O} & 1.349035 & -1.529866 & 0.058549 \\ \mathrm{O} & 1.635554 & -0.077235 & -2.056321 \\ \mathrm{O} & 0.257518 & 1.293684 & -3.904727 \\ \mathrm{O} & 0.149674 & -1.365305 & -3.92965 \\ \mathrm{O} & 0.22302 & 1.335987 & 3.951472 \\ \mathrm{O} & 1.402598 & 1.478044 & -0.04549 \\ \mathrm{O} & -1.060146 & 2.066204 & -0.038756 \\ \mathrm{O} & -1.740967 & 2.503493 & 2.625667 \\ \mathrm{O} & 0.153953 & 3.966845 & 1.190615 \\ \mathrm{O} & -3.317341 & 1.426472 & 1.413594 \\ \mathrm{O} & 0.059377 & -3.985545 & -1.180639 \\ \mathrm{O} & 0.307541 & -4.062767 & 1.436743 \\ \mathrm{O} & -1.129493 & -2.07616 & 0.077079 \\ \mathrm{O} & -1.844765 & -2.465747 & -2.592962 \\ \mathrm{O} & -1.838188 & -2.439442 & 2.672987 \\ \mathrm{O} & 0.250132 & -1.325379 & 3.928732 \\ \mathrm{O} & 1.651897 & 0.007275 & 2.067478 \\ \mathrm{O} & -0.803241 & 0.052043 & 1.541333 \\ \mathrm{Zr} & 2.79497 & -0.05057 & 0.000491 \\ \mathrm{Zr} & 0.232903 & 1.704776 & -1.833307 \\ \mathrm{Zr} & 0.247917 & -1.864354 & -1.648529 \\ \mathrm{Zr} & 0.194296 & -1.738065 & 1.858078 \\ \mathrm{Zr} & -2.205628 & 0.020941 & 0.021012 \\ \mathrm{Zr} & 0.316169 & 1.829335 & 1.667604 \\ \mathrm{C} & -3.128847 & -5.311065 & -0.361256 \\ \mathrm{H} & -3.069037 & -5.570693 & -1.429928 \\ \mathrm{H} & -2.656054 & -6.147609 & 0.173797 \\ \mathrm{Zn} & -1.947227 & -3.780725 & -0.198356 \\ \mathrm{Zn} & -1.828162 & 3.804924 & 0.228112 \\ \mathrm{C} & -3.12933 & 5.23213 & 0.018475 \\ \mathrm{H} & -4.061431 & 4.789955 & -0.363577 \\ \mathrm{H} & -3.372928 & 5.629773 & 1.014465 \\ \mathrm{C} & -4.584763 & -5.168505 & 0.061641 \\ \mathrm{H} & -4.687847 & -5.120763 & 1.152569 \\ \mathrm{H} & -5.050273 & -4.258389 & -0.340474 \\ \mathrm{H} & -5.196024 & -6.016653 & -0.283807 \\ \mathrm{C} & -2.658764 & 6.350381 & -0.904863 \\ \mathrm{H} & -2.419141 & 5.976757 & -1.90925 \\ \mathrm{H} & -3.422086 & 7.133709 & -1.030458 \\ \mathrm{H} & -1.752366 & 6.843341 & -0.527178\end{array}$


Table S19. Structure for a ZnEt group added to two HB sites on the node that are adjacent of each other.

$E=-5470.6790882$ Hartrees, $G=-5469.928901$ Hartrees

\begin{tabular}{lrrr}
\hline Atom & $\mathrm{X}$ & $\mathrm{Y}$ & \multicolumn{1}{c}{$\mathrm{Z}$} \\
\hline \hline $\mathrm{C}$ & -6.263955 & 1.977571 & -2.795373 \\
$\mathrm{C}$ & 5.391015 & 3.759851 & 1.203222 \\
$\mathrm{C}$ & -5.902584 & 2.955967 & 2.235819 \\
$\mathrm{C}$ & 5.007724 & 2.859888 & -3.66292 \\
$\mathrm{C}$ & -4.977292 & 2.248166 & -3.284152 \\
$\mathrm{C}$ & 4.051485 & 3.999067 & 1.544747 \\
$\mathrm{C}$ & -4.570771 & 3.370264 & 2.388318 \\
$\mathrm{C}$ & 3.632438 & 2.977295 & -3.913395 \\
$\mathrm{C}$ & -4.829311 & 3.157677 & -4.342191 \\
$\mathrm{C}$ & 3.72921 & 5.155712 & 2.270083 \\
$\mathrm{C}$ & -4.308449 & 4.577114 & 3.053568 \\
$\mathrm{C}$ & 3.199837 & 3.802629 & -4.961576 \\
$\mathrm{C}$ & -5.942135 & 3.793879 & -4.887332 \\
$\mathrm{C}$ & 4.721039 & 6.067697 & 2.618009 \\
$\mathrm{C}$ & -5.353415 & 5.367023 & 3.52501 \\
$\mathrm{C}$ & 4.120579 & 4.517576 & -5.72261 \\
$\mathrm{C}$ & -7.237662 & 3.532114 & -4.39991 \\
$\mathrm{C}$ & 6.063956 & 5.854399 & 2.250387 \\
$\mathrm{C}$ & -6.695041 & 4.973071 & 3.3528 \\
$\mathrm{C}$ & 5.502325 & 4.424968 & -5.463289 \\
$\mathrm{C}$ & -7.375187 & 2.606034 & -3.348075 \\
$\mathrm{C}$ & 6.380628 & 4.672291 & 1.555129 \\
$\mathrm{C}$ & -6.945362 & 3.742789 & 2.715022 \\
$\mathrm{C}$ & 5.926947 & 3.568329 & -4.430054 \\
$\mathrm{C}$ & 5.817535 & -1.78809 & 2.214517 \\
$\mathrm{C}$ & -5.809842 & -3.435621 & -1.805835 \\
$\mathrm{C}$ & 5.50758 & -2.689197 & -2.665823 \\
$\mathrm{C}$ & -5.171467 & -5.764529 & -3.198568 \\
$\mathrm{C}$ & & &
\end{tabular}




\begin{tabular}{|c|c|c|c|}
\hline C & 4.93065 & -5.082461 & -3.97648 \\
\hline C & -4.56398 & -4.181544 & 5.243472 \\
\hline C & 6.811855 & -3.252652 & 3.888112 \\
\hline C & -6.505881 & -5.550207 & -2.800207 \\
\hline C & 6.274038 & -4.682421 & -3.837989 \\
\hline C & -5.945221 & -4.10234 & 4.977869 \\
\hline C & 6.938963 & -2.356889 & 2.809702 \\
\hline C & -6.806436 & -4.356355 & -2.115732 \\
\hline C & 6.53941 & -3.461196 & -3.190683 \\
\hline C & -6.375263 & -3.214347 & 3.972981 \\
\hline C & -3.471666 & 2.538453 & 1.814983 \\
\hline C & -3.789109 & 1.626764 & -2.636067 \\
\hline C & -3.41826 & -2.700009 & -1.782015 \\
\hline C & 3.071119 & -2.320203 & -2.175856 \\
\hline$C$ & 3.339524 & -1.496343 & 2.020184 \\
\hline C & 2.980393 & 3.052884 & 1.128006 \\
\hline C & -3.115071 & -1.783692 & 2.680023 \\
\hline C & 2.659682 & 2.229877 & -3.063913 \\
\hline $\mathrm{H}$ & -6.372199 & 1.276822 & -1.970604 \\
\hline $\mathrm{H}$ & 5.636514 & 2.853908 & 0.653122 \\
\hline $\mathrm{H}$ & -6.102391 & 2.016199 & 1.726259 \\
\hline $\mathrm{H}$ & 5.337245 & 2.210065 & -2.855415 \\
\hline $\mathrm{H}$ & -3.829451 & 3.375893 & -4.711253 \\
\hline $\mathrm{H}$ & 2.692191 & 5.327151 & 2.551191 \\
\hline $\mathrm{H}$ & -3.274983 & 4.897985 & 3.169358 \\
\hline $\mathrm{H}$ & 2.13428 & 3.873863 & -5.170478 \\
\hline $\mathrm{H}$ & -5.805627 & 4.506124 & -5.699918 \\
\hline $\mathrm{H}$ & 4.451706 & 6.963355 & 3.176256 \\
\hline $\mathrm{H}$ & -5.127609 & 6.308473 & 4.023964 \\
\hline $\mathrm{H}$ & 3.765457 & 5.158542 & -6.528399 \\
\hline $\mathrm{H}$ & -8.365938 & 2.384088 & -2.953492 \\
\hline $\mathrm{H}$ & 7.414332 & 4.473746 & 1.274753 \\
\hline $\mathrm{H}$ & -7.973047 & 3.40696 & 2.581022 \\
\hline $\mathrm{H}$ & 6.990597 & 3.468268 & -4.217561 \\
\hline $\mathrm{H}$ & 5.915503 & -1.111535 & 1.368337 \\
\hline $\mathrm{H}$ & -6.042686 & -2.52601 & -1.257082 \\
\hline $\mathrm{H}$ & 5.716143 & -1.755989 & -2.146977 \\
\hline $\mathrm{H}$ & -5.79821 & -1.782891 & 2.460158 \\
\hline $\mathrm{H}$ & 3.398253 & -3.174404 & 4.15965 \\
\hline $\mathrm{H}$ & -3.142574 & -5.024737 & -3.189874 \\
\hline $\mathrm{H}$ & 2.861003 & -4.627906 & -3.558087 \\
\hline $\mathrm{H}$ & -2.582778 & -3.489809 & 4.726636 \\
\hline $\mathrm{H}$ & 5.393961 & -4.206058 & 5.217892 \\
\hline
\end{tabular}




$\begin{array}{rrrr}\mathrm{H} & -4.910879 & -6.672866 & -3.740251 \\ \mathrm{H} & 4.697321 & -6.015342 & -4.488135 \\ \mathrm{H} & -4.202145 & -4.859173 & 6.015682 \\ \mathrm{H} & 7.929215 & -2.11661 & 2.424553 \\ \mathrm{H} & -7.832497 & -4.156015 & -1.809579 \\ \mathrm{H} & 7.56859 & -3.12055 & -3.084891 \\ \mathrm{H} & -7.43808 & -3.129048 & 3.749836 \\ \mathrm{H} & -7.511772 & 5.593461 & 3.714274 \\ \mathrm{H} & 6.836902 & 6.572073 & 2.515293 \\ \mathrm{H} & -7.281968 & -6.276861 & -3.028492 \\ \mathrm{H} & -6.657634 & -4.707192 & 5.533914 \\ \mathrm{H} & 7.081937 & -5.291336 & -4.236905 \\ \mathrm{H} & 6.220782 & 4.986625 & -6.05576 \\ \mathrm{H} & 7.688566 & -3.706165 & 4.344646 \\ \mathrm{H} & -8.106093 & 4.030902 & -4.823819 \\ \mathrm{H} & 2.195033 & 0.792784 & 2.098758 \\ \mathrm{H} & -2.408342 & 2.800511 & -0.643857 \\ \mathrm{H} & -1.350713 & -2.283038 & -4.042773 \\ \mathrm{H} & -0.505819 & -0.939322 & -4.172216 \\ \mathrm{H} & -0.685601 & -0.61627 & 4.371119 \\ \mathrm{H} & 0.463787 & -4.5718 & -1.561282 \\ \mathrm{H} & 0.585319 & -3.662834 & 2.820522 \\ \mathrm{H} & 1.540287 & -0.322753 & -3.193201 \\ \mathrm{H} & -1.766117 & -2.881017 & 0.514289 \\ \mathrm{H} & -1.406787 & 0.902277 & -4.768598 \\ \mathrm{H} & -0.941078 & 2.57966 & 3.670867 \\ \mathrm{H} & 0.250259 & 4.072385 & -2.689512 \\ \mathrm{H} & 0.249055 & 4.82154 & 0.524449 \\ \mathrm{H} & -0.552718 & 4.065158 & -1.313375 \\ \mathrm{O} & -1.36687 & -0.257617 & -1.586736 \\ \mathrm{O} & -3.772857 & -1.74795 & -1.0234 \\ \mathrm{O} & -3.592941 & -1.162338 & 1.6789 \\ \mathrm{O} & -4.004313 & 0.912971 & -1.607344 \\ \mathrm{O} & -2.644529 & 1.879069 & -3.130081 \\ \mathrm{O} & -0.594874 & 3.790254 & -2.301157 \\ \mathrm{O} & 1.421063 & 2.41567 & -3.273371 \\ \mathrm{O} & 1.78666 & 3.352192 & 1.42279 \\ \mathrm{O} & 3.149199 & 1.45408 & -2.183624 \\ \mathrm{O} & 3.348958 & 1.998862 & 0.510855 \\ \mathrm{O} & 3.39917 & -1.241153 & -1.594302 \\ \mathrm{O} & 3.561199 & -0.693973 & 1.057804 \\ \mathrm{O} & 2.197612 & -1.796187 & 2.483331 \\ \mathrm{O} & 1.884323 & -2.760511 & -2.309889\end{array}$




$\begin{array}{lrrr}\mathrm{O} & 1.023994 & -1.352774 & -0.013631 \\ \mathrm{O} & 1.013864 & -0.212916 & -2.387937 \\ \mathrm{O} & -0.578096 & 0.662018 & -4.32894 \\ \mathrm{O} & -0.457231 & -1.926945 & -3.901183 \\ \mathrm{O} & -0.052234 & 2.475589 & 3.300873 \\ \mathrm{O} & 0.709759 & 1.62904 & -0.643357 \\ \mathrm{O} & -1.780086 & 2.069079 & -0.556365 \\ \mathrm{O} & -2.281584 & 2.906126 & 2.028218 \\ \mathrm{O} & -0.48109 & 4.238382 & 0.271369 \\ \mathrm{O} & -3.823991 & 1.521573 & 1.132409 \\ \mathrm{O} & 0.01508 & -4.025596 & -0.896588 \\ \mathrm{O} & 0.139934 & -3.410005 & 1.996542 \\ \mathrm{O} & -1.392433 & -2.012065 & 0.298446 \\ \mathrm{O} & -2.236299 & -2.896011 & -2.215026 \\ \mathrm{O} & -1.894946 & -1.808474 & 3.016912 \\ \mathrm{O} & 0.188795 & -0.549624 & 3.959461 \\ \mathrm{O} & 1.323627 & 0.662861 & 1.687819 \\ \mathrm{O} & -1.148998 & 0.374758 & 1.274916 \\ \mathrm{Zr} & 2.259435 & 0.303816 & -0.478042 \\ \mathrm{Zr} & -0.515817 & 1.484524 & -2.383389 \\ \mathrm{Zr} & -0.180784 & -1.992402 & -1.610311 \\ \mathrm{Zr} & 0.034566 & -1.227054 & 1.912341 \\ \mathrm{Zr} & -2.712606 & -0.032705 & -0.020769 \\ \mathrm{Zr} & -0.239936 & 2.327682 & 1.13622 \\ \mathrm{C} & 3.304381 & -4.501792 & 0.45495 \\ \mathrm{H} & 3.907303 & -3.691992 & 0.012884 \\ \mathrm{H} & 3.706364 & -4.629375 & 1.47044 \\ \mathrm{Zn} & 1.457425 & -3.849569 & 0.555935 \\ \mathrm{C} & 3.492746 & -5.788129 & -0.341242 \\ \mathrm{H} & 4.554775 & -6.033569 & -0.501339 \\ \mathrm{H} & 3.036235 & -6.649933 & 0.164297 \\ \mathrm{H} & 3.038283 & -5.73167 & -1.340334 \\ \mathrm{Zn} & 1.206598 & 1.165647 & 4.130923 \\ \mathrm{C} & 3.093152 & 1.501825 & 4.563644 \\ \mathrm{H} & 3.482874 & 0.719834 & 5.22972 \\ \mathrm{H} & 3.657044 & 1.369927 & 3.620929 \\ \mathrm{C} & 3.385969 & 2.887358 & 5.128555 \\ \mathrm{H} & 2.980281 & 3.004639 & 6.142197 \\ \mathrm{H} & 4.464792 & 3.101535 & 5.186487 \\ \mathrm{H} & 2.938546 & 3.681664 & 4.516457\end{array}$


Table S20. Structure for $2 \mathrm{H}_{2} \mathrm{O}-2 \mathrm{Et}-\mathrm{A}$.

$E=-5470.64865903$ Hartrees, $G=-5469.899981$ Hartrees

\begin{tabular}{|c|c|c|c|}
\hline Atom & $\mathrm{X}$ & $\mathrm{Y}$ & Z \\
\hline$C$ & -6.170251 & 2.427211 & -1.652384 \\
\hline$C$ & 5.522876 & 3.497345 & 2.486346 \\
\hline $\mathrm{C}$ & -5.712839 & 2.05348 & 3.451748 \\
\hline C & 5.045349 & 3.902204 & -2.43756 \\
\hline $\mathrm{C}$ & -4.904761 & 2.862093 & -2.072968 \\
\hline$C$ & 4.185763 & 3.590728 & 2.900626 \\
\hline$C$ & -4.390551 & 2.459243 & 3.686835 \\
\hline C & 3.661392 & 4.033299 & -2.625687 \\
\hline $\mathrm{C}$ & -4.810046 & 4.02361 & -2.854403 \\
\hline C & 3.847607 & 4.502583 & 3.911542 \\
\hline$C$ & -4.147452 & 3.455901 & 4.643754 \\
\hline$C$ & 3.178633 & 5.090545 & -3.41067 \\
\hline $\mathrm{C}$ & -5.954408 & 4.7418 & -3.193452 \\
\hline$C$ & 4.820413 & 5.324423 & 4.472541 \\
\hline$C$ & -5.203244 & 4.056023 & 5.324436 \\
\hline$C$ & 4.058623 & 6.013019 & -3.969913 \\
\hline$C$ & -7.229102 & 4.315261 & -2.771902 \\
\hline$C$ & 6.159476 & 5.263095 & 4.039862 \\
\hline$C$ & -6.536517 & 3.674778 & 5.075731 \\
\hline$C$ & 5.448525 & 5.903721 & -3.766774 \\
\hline$C$ & -7.312982 & 3.139953 & -2.000855 \\
\hline$C$ & 6.493537 & 4.318558 & 3.051275 \\
\hline$C$ & -6.76617 & 2.648731 & 4.138982 \\
\hline$C$ & 5.923859 & 4.819974 & -3.004373 \\
\hline$C$ & 6.138177 & -2.102041 & 1.984952 \\
\hline$C$ & -5.531896 & -3.035735 & -2.139594 \\
\hline$C$ & 5.733619 & -1.692067 & -2.954139 \\
\hline$C$ & -5.08858 & -3.410605 & 2.990008 \\
\hline$C$ & 4.875103 & -2.56413 & 2.38356 \\
\hline$C$ & -4.204042 & -3.118786 & -2.585717 \\
\hline C & 4.412292 & -2.118741 & -3.154144 \\
\hline$C$ & -3.705234 & -3.522077 & 3.199556 \\
\hline$C$ & 4.791649 & -3.691337 & 3.213558 \\
\hline$C$ & -3.883575 & -4.043373 & -3.591563 \\
\hline C & 4.151998 & -3.104128 & -4.117963 \\
\hline C & -3.216607 & -4.609954 & 3.939043 \\
\hline $\mathrm{C}$ & 5.945053 & -4.360933 & 3.610643 \\
\hline $\mathrm{C}$ & -4.859847 & -4.890014 & -4.109634 \\
\hline$C$ & 5.195212 & -3.672682 & -4.842439 \\
\hline
\end{tabular}




\begin{tabular}{|c|c|c|c|}
\hline C & -4.089485 & -5.573453 & 4.439208 \\
\hline C & 7.216677 & -3.920997 & 3.1944 \\
\hline C & -6.189684 & -4.835506 & -3.647194 \\
\hline C & 6.529225 & -3.276573 & -4.624687 \\
\hline C & -5.478645 & -5.475414 & 4.226429 \\
\hline C & 7.290384 & -2.768255 & 2.389867 \\
\hline C & -6.508254 & -3.876231 & -2.666162 \\
\hline C & 6.774422 & -2.261239 & -3.681402 \\
\hline C & -5.959611 & -4.36913 & 3.499742 \\
\hline C & -3.282012 & 1.848361 & 2.896166 \\
\hline C & -3.682651 & 2.133724 & -1.632177 \\
\hline C & -3.163269 & -2.248836 & -1.96028 \\
\hline C & 3.300033 & -1.551251 & -2.344653 \\
\hline C & 3.648677 & -1.8569 & 1.914724 \\
\hline C & 3.1332 & 2.751501 & 2.265524 \\
\hline C & -2.776126 & -2.535035 & 2.579509 \\
\hline C & 2.732412 & 3.054469 & -1.988697 \\
\hline $\mathrm{H}$ & -6.237334 & 1.527655 & -1.044672 \\
\hline $\mathrm{H}$ & 5.780417 & 2.779887 & 1.710627 \\
\hline $\mathrm{H}$ & -5.897267 & 1.276608 & 2.713654 \\
\hline $\mathrm{H}$ & 5.413211 & 3.073411 & -1.837302 \\
\hline $\mathrm{H}$ & -3.827193 & 4.364274 & -3.172616 \\
\hline $\mathrm{H}$ & 2.81407 & 4.557444 & 4.245273 \\
\hline $\mathrm{H}$ & -3.122016 & 3.772032 & 4.823648 \\
\hline $\mathrm{H}$ & 2.106778 & 5.177691 & -3.575173 \\
\hline $\mathrm{H}$ & -5.85958 & 5.646132 & -3.792608 \\
\hline $\mathrm{H}$ & 4.538625 & 6.031937 & 5.251059 \\
\hline $\mathrm{H}$ & -4.993127 & 4.839558 & 6.050908 \\
\hline $\mathrm{H}$ & 3.664861 & 6.831233 & -4.571038 \\
\hline $\mathrm{H}$ & -8.286497 & 2.785957 & -1.664605 \\
\hline $\mathrm{H}$ & 7.525084 & 4.237313 & 2.711642 \\
\hline $\mathrm{H}$ & -7.786056 & 2.323948 & 3.937402 \\
\hline $\mathrm{H}$ & 6.994764 & 4.704331 & -2.843579 \\
\hline $\mathrm{H}$ & 6.196487 & -1.223777 & 1.346264 \\
\hline $\mathrm{H}$ & -5.778633 & -2.307695 & -1.370462 \\
\hline $\mathrm{H}$ & 5.926702 & -0.922358 & -2.210153 \\
\hline $\mathrm{H}$ & -5.462706 & -2.568833 & 2.412168 \\
\hline $\mathrm{H}$ & 3.807988 & -4.040761 & 3.522022 \\
\hline $\mathrm{H}$ & -2.855495 & -4.10231 & -3.942803 \\
\hline $\mathrm{H}$ & 3.12438 & -3.421819 & -4.279461 \\
\hline $\mathrm{H}$ & -2.143776 & -4.698578 & 4.097805 \\
\hline $\mathrm{H}$ & 5.860115 & -5.243142 & 4.243433 \\
\hline $\mathrm{H}$ & -4.586989 & -5.610836 & -4.87909 \\
\hline
\end{tabular}




\begin{tabular}{|c|c|c|c|}
\hline $\mathrm{H}$ & 4.97705 & -4.443955 & -5.579837 \\
\hline $\mathrm{H}$ & -3.688571 & -6.416146 & 5.000462 \\
\hline $\mathrm{H}$ & 8.262908 & -2.400619 & 2.065173 \\
\hline $\mathrm{H}$ & -7.532085 & -3.798965 & -2.302691 \\
\hline $\mathrm{H}$ & 7.795553 & -1.926076 & -3.504984 \\
\hline $\mathrm{H}$ & -7.029487 & -4.264946 & 3.324632 \\
\hline $\mathrm{H}$ & -7.361721 & 4.148528 & 5.60178 \\
\hline $\mathrm{H}$ & 6.916946 & 5.91221 & 4.472438 \\
\hline $\mathrm{H}$ & -6.949592 & -5.501486 & -4.048528 \\
\hline $\mathrm{H}$ & -6.158866 & -6.229635 & 4.614436 \\
\hline $\mathrm{H}$ & 7.344464 & -3.729352 & -5.183883 \\
\hline $\mathrm{H}$ & 6.135004 & 6.626515 & -4.20096 \\
\hline $\mathrm{H}$ & 8.117454 & -4.448336 & 3.499358 \\
\hline $\mathrm{H}$ & -8.122114 & 4.876543 & -3.035907 \\
\hline $\mathrm{H}$ & 2.193186 & 0.26048 & 2.889355 \\
\hline $\mathrm{H}$ & -2.281364 & 2.787819 & 0.549132 \\
\hline $\mathrm{H}$ & -0.486692 & -1.845038 & -4.263624 \\
\hline $\mathrm{H}$ & -0.137674 & -1.86132 & 4.555572 \\
\hline $\mathrm{H}$ & -0.226264 & -4.223536 & 1.235689 \\
\hline $\mathrm{H}$ & 0.788762 & -4.032101 & -2.185722 \\
\hline $\mathrm{H}$ & 1.551781 & 0.553642 & -2.851307 \\
\hline $\mathrm{H}$ & -1.632906 & -2.841766 & 0.076068 \\
\hline $\mathrm{H}$ & -1.105774 & 2.556271 & -3.971378 \\
\hline $\mathrm{H}$ & 0.272528 & 0.239619 & 4.301545 \\
\hline $\mathrm{H}$ & -0.764727 & 1.397724 & 4.655684 \\
\hline $\mathrm{H}$ & 0.296609 & 4.654895 & -1.075863 \\
\hline $\mathrm{H}$ & 0.383858 & 4.482576 & 2.179167 \\
\hline $\mathrm{H}$ & -0.487915 & 4.254326 & 0.252269 \\
\hline 0 & -1.244545 & 0.144154 & -1.283372 \\
\hline 0 & -3.539214 & -1.522645 & -0.985625 \\
\hline 0 & -3.297098 & -1.663513 & 1.813981 \\
\hline 0 & -3.853448 & 1.171075 & -0.822341 \\
\hline 0 & -2.557067 & 2.560244 & -2.045817 \\
\hline 0 & -0.534969 & 4.252917 & -0.772483 \\
\hline 0 & 1.481428 & 3.254389 & -2.123446 \\
\hline 0 & 1.935808 & 2.901616 & 2.665411 \\
\hline 0 & 3.25838 & 2.097744 & -1.346388 \\
\hline 0 & 3.513736 & 1.942532 & 1.362898 \\
\hline 0 & 3.608185 & -0.631239 & -1.521328 \\
\hline 0 & 3.834465 & -0.823457 & 1.191306 \\
\hline 0 & 2.526183 & -2.316117 & 2.269493 \\
\hline 0 & 2.141072 & -2.027186 & -2.521524 \\
\hline 0 & 1.260095 & -1.217351 & 0.046159 \\
\hline
\end{tabular}




$\begin{array}{lrrr}\mathrm{O} & 1.148274 & 0.468388 & -1.973288 \\ \mathrm{O} & -0.522891 & 1.869905 & -3.609545 \\ \mathrm{O} & -0.13656 & -1.069568 & -3.79811 \\ \mathrm{O} & 0.123768 & 1.255276 & 4.288184 \\ \mathrm{O} & 0.863247 & 1.770817 & 0.20995 \\ \mathrm{O} & -1.626541 & 2.082431 & 0.441785 \\ \mathrm{O} & -2.088795 & 2.167751 & 3.190788 \\ \mathrm{O} & -0.368137 & 3.98326 & 1.828288 \\ \mathrm{O} & -3.620901 & 1.05873 & 1.958192 \\ \mathrm{O} & 0.335915 & -3.654926 & -1.414945 \\ \mathrm{O} & 0.592768 & -3.787044 & 1.509508 \\ \mathrm{O} & -1.14536 & -2.013461 & 0.187198 \\ \mathrm{O} & -1.980255 & -2.332291 & -2.405301 \\ \mathrm{O} & -1.540241 & -2.654216 & 2.833032 \\ \mathrm{O} & 0.483454 & -1.319255 & 4.048924 \\ \mathrm{O} & 1.540649 & 0.259492 & 2.173858 \\ \mathrm{O} & -0.927598 & -0.001331 & 1.728959 \\ \mathrm{Zr} & 2.480012 & 0.479129 & 0.01453 \\ \mathrm{Zr} & -0.380936 & 2.053639 & -1.464812 \\ \mathrm{Zr} & 0.070088 & -1.523318 & -1.666465 \\ \mathrm{Zr} & 0.336044 & -1.677535 & 1.979181 \\ \mathrm{Zr} & -2.497866 & -0.11397 & 0.404503 \\ \mathrm{Zr} & -0.051395 & 1.910104 & 2.088701 \\ \mathrm{C} & 3.706762 & -4.269692 & -0.525105 \\ \mathrm{H} & 4.341799 & -3.38471 & -0.356725 \\ \mathrm{H} & 3.701814 & -4.410181 & -1.61639 \\ \mathrm{Zn} & 1.888848 & -3.781391 & 0.012206 \\ \mathrm{C} & 4.325143 & -5.48772 & 0.149443 \\ \mathrm{H} & 3.780513 & -6.410026 & -0.093302 \\ \mathrm{H} & 5.371765 & -5.644593 & -0.155413 \\ \mathrm{H} & 4.327321 & -5.400628 & 1.242854 \\ \mathrm{Zn} & -1.701867 & 0.187934 & -3.791498 \\ \mathrm{C} & -3.578276 & 0.176716 & -4.360018 \\ \mathrm{H} & -3.808407 & -0.764895 & -4.879487 \\ \mathrm{H} & -4.186758 & 0.155399 & -3.441783 \\ \mathrm{H} & -3.97903 & 1.365699 & -5.225733 \\ \mathrm{H} & -5.062446 & 1.405666 & -5.42043 \\ \mathrm{H} & & 2.323905 & -4.754773\end{array}$


Table S21. Structure for a $\mathrm{ZnEt}$ group added to two $\mu \mathrm{OH}$ sites on the node that are adjacent of each other.

$E=-5470.66907846$ Hartrees, $G=-5469.918823$ Hartrees

\begin{tabular}{|c|c|c|c|}
\hline Atom & $X$ & $\mathrm{Y}$ & Z \\
\hline $\mathrm{C}$ & 5.540887 & 3.784394 & 1.892579 \\
\hline $\mathrm{C}$ & -6.197655 & 2.206803 & -1.944323 \\
\hline $\mathrm{C}$ & 5.051029 & 3.281423 & -3.197462 \\
\hline $\mathrm{C}$ & -5.69958 & 2.743322 & 2.964917 \\
\hline $\mathrm{C}$ & 4.221228 & 3.931277 & 2.344895 \\
\hline $\mathrm{C}$ & -4.92524 & 2.591115 & -2.393153 \\
\hline$C$ & 3.666255 & 3.3824 & -3.398944 \\
\hline C & -4.374878 & 3.179152 & 3.117053 \\
\hline $\mathrm{C}$ & 3.890907 & 5.048465 & 3.126755 \\
\hline C & -4.822866 & 3.54785 & -3.413936 \\
\hline $\mathrm{C}$ & 3.184568 & 4.293404 & -4.350854 \\
\hline $\mathrm{C}$ & -4.119169 & 4.322786 & 3.887586 \\
\hline C & 4.85564 & 6.00481 & 3.434985 \\
\hline C & -5.967291 & 4.129598 & -3.950922 \\
\hline $\mathrm{C}$ & 4.063799 & 5.107577 & -5.059546 \\
\hline C & -5.167592 & 5.031392 & 4.467912 \\
\hline$C$ & 6.182295 & 5.868264 & 2.981301 \\
\hline $\mathrm{C}$ & -7.248264 & 3.776244 & -3.48368 \\
\hline $\mathrm{C}$ & 5.454171 & 5.033058 & -4.84477 \\
\hline$C$ & -6.503287 & 4.615358 & 4.300942 \\
\hline C & 6.505461 & 4.735164 & 2.210235 \\
\hline $\mathrm{C}$ & -7.339946 & 2.788401 & -2.485278 \\
\hline $\mathrm{C}$ & 5.928778 & 4.090257 & -3.912628 \\
\hline C & -6.745325 & 3.447698 & 3.552815 \\
\hline C & -5.543649 & -3.385925 & -1.418215 \\
\hline $\mathrm{C}$ & 6.141808 & -1.680477 & 2.404836 \\
\hline $\mathrm{C}$ & -5.117461 & -2.860496 & 3.508106 \\
\hline C & 5.665319 & -2.181643 & -2.710988 \\
\hline C & -4.219869 & -3.559542 & -1.848602 \\
\hline $\mathrm{C}$ & 4.876937 & -2.052421 & 2.885218 \\
\hline $\mathrm{C}$ & -3.729856 & -2.982335 & 3.674574 \\
\hline $\mathrm{C}$ & 4.336334 & -2.598386 & -2.884546 \\
\hline C & -3.909247 & -4.646261 & -2.678674 \\
\hline $\mathrm{C}$ & 4.794484 & -3.017708 & 3.900509 \\
\hline C & -3.233734 & -3.878501 & 4.632904 \\
\hline $\mathrm{C}$ & 4.082824 & -3.772841 & -3.609425 \\
\hline $\mathrm{C}$ & -4.895008 & -5.557653 & -3.044753 \\
\hline
\end{tabular}




\begin{tabular}{|c|c|c|c|}
\hline C & 5.946721 & -3.623168 & 4.394545 \\
\hline C & -4.106618 & -4.658772 & 5.385015 \\
\hline$C$ & 5.134882 & -4.522607 & -4.130424 \\
\hline$C$ & -6.221776 & -5.407394 & -2.596491 \\
\hline$C$ & 7.219472 & -3.278722 & 3.89781 \\
\hline C & -5.500237 & -4.569693 & 5.201179 \\
\hline$C$ & 6.472587 & -4.117646 & -3.953777 \\
\hline$C$ & -6.529189 & -4.293807 & -1.792166 \\
\hline$C$ & 7.292978 & -2.279879 & 2.907338 \\
\hline C & -5.987784 & -3.64083 & 4.262922 \\
\hline$C$ & 6.714233 & -2.927022 & -3.241538 \\
\hline$C$ & 2.740797 & 2.546904 & -2.5788 \\
\hline$C$ & 3.180714 & 2.946881 & 1.93677 \\
\hline C & 3.653977 & -1.439445 & 2.285766 \\
\hline$C$ & -2.791751 & -2.188428 & 2.835643 \\
\hline C & -3.169481 & -2.594679 & -1.412805 \\
\hline$C$ & -3.69732 & 2.010706 & -1.784101 \\
\hline C & 3.227276 & -1.837219 & -2.242227 \\
\hline$C$ & -3.268235 & 2.425729 & 2.458013 \\
\hline $\mathrm{H}$ & 5.789313 & 2.920766 & 1.279663 \\
\hline $\mathrm{H}$ & -6.270099 & 1.455259 & -1.16106 \\
\hline $\mathrm{H}$ & 5.420614 & 2.569065 & -2.46349 \\
\hline $\mathrm{H}$ & -5.8893 & 1.84972 & 2.374524 \\
\hline $\mathrm{H}$ & 2.863797 & 5.166864 & 3.465078 \\
\hline $\mathrm{H}$ & -3.835454 & 3.82733 & -3.774753 \\
\hline $\mathrm{H}$ & 2.110139 & 4.376342 & -4.502137 \\
\hline $\mathrm{H}$ & -3.089071 & 4.645053 & 4.025371 \\
\hline $\mathrm{H}$ & 4.576844 & 6.871712 & 4.032561 \\
\hline $\mathrm{H}$ & -5.869044 & 4.876542 & -4.737694 \\
\hline $\mathrm{H}$ & 3.66737 & 5.820934 & -5.780962 \\
\hline $\mathrm{H}$ & -4.950183 & 5.921041 & 5.057557 \\
\hline $\mathrm{H}$ & 7.524345 & 4.604774 & 1.847555 \\
\hline $\mathrm{H}$ & -8.319416 & 2.483579 & -2.118713 \\
\hline $\mathrm{H}$ & 7.000315 & 4.001978 & -3.73666 \\
\hline $\mathrm{H}$ & -7.768063 & 3.096782 & 3.420125 \\
\hline $\mathrm{H}$ & -5.778538 & -2.533506 & -0.784968 \\
\hline $\mathrm{H}$ & 6.201682 & -0.930336 & 1.619514 \\
\hline $\mathrm{H}$ & -5.494275 & -2.153676 & 2.77207 \\
\hline $\mathrm{H}$ & 5.857615 & -1.275295 & -2.140827 \\
\hline $\mathrm{H}$ & -2.887315 & -4.764174 & -3.034821 \\
\hline $\mathrm{H}$ & 3.813739 & -3.307545 & 4.273402 \\
\hline $\mathrm{H}$ & -2.15761 & -3.954 & 4.770876 \\
\hline $\mathrm{H}$ & 3.052107 & -4.100723 & -3.730981 \\
\hline
\end{tabular}




\begin{tabular}{|c|c|c|c|}
\hline $\mathrm{H}$ & -4.635069 & -6.399188 & -3.685478 \\
\hline $\mathrm{H}$ & 5.859332 & -4.384005 & 5.169037 \\
\hline $\mathrm{H}$ & -3.704973 & -5.355329 & 6.120053 \\
\hline $\mathrm{H}$ & 4.917939 & -5.439698 & -4.676774 \\
\hline $\mathrm{H}$ & -7.551575 & -4.146215 & -1.446649 \\
\hline $\mathrm{H}$ & 8.264298 & -1.984424 & 2.512835 \\
\hline $\mathrm{H}$ & -7.062219 & -3.539288 & 4.114794 \\
\hline $\mathrm{H}$ & 7.738923 & -2.588982 & -3.091607 \\
\hline $\mathrm{H}$ & 6.140609 & 5.674307 & -5.392757 \\
\hline $\mathrm{H}$ & -8.141461 & 4.23856 & -3.897427 \\
\hline $\mathrm{H}$ & 8.117672 & -3.759125 & 4.278296 \\
\hline $\mathrm{H}$ & 7.293369 & -4.706166 & -4.356931 \\
\hline $\mathrm{H}$ & -6.18103 & -5.186701 & 5.783142 \\
\hline $\mathrm{H}$ & -7.322146 & 5.171397 & 4.751571 \\
\hline $\mathrm{H}$ & -6.990698 & -6.122342 & -2.879667 \\
\hline $\mathrm{H}$ & 6.934715 & 6.616518 & 3.219428 \\
\hline $\mathrm{H}$ & 1.618445 & 3.265678 & -0.228405 \\
\hline $\mathrm{H}$ & 1.523923 & -0.848079 & 4.403696 \\
\hline $\mathrm{H}$ & 0.428257 & 0.284236 & 4.232106 \\
\hline $\mathrm{H}$ & -0.041708 & -1.926315 & -4.269589 \\
\hline $\mathrm{H}$ & 0.112281 & -3.882694 & 0.104433 \\
\hline $\mathrm{H}$ & 0.470057 & -4.22565 & 2.346986 \\
\hline $\mathrm{H}$ & -0.705258 & -4.192212 & -1.207342 \\
\hline $\mathrm{H}$ & -1.70529 & 0.244458 & 3.202948 \\
\hline $\mathrm{H}$ & 0.971772 & 2.374515 & 4.333162 \\
\hline $\mathrm{H}$ & -0.21462 & 0.315571 & -3.927649 \\
\hline $\mathrm{H}$ & 0.366434 & 1.749578 & -4.247085 \\
\hline $\mathrm{H}$ & -1.264361 & 4.515274 & 1.522458 \\
\hline $\mathrm{H}$ & -1.434111 & 4.328273 & -1.73157 \\
\hline $\mathrm{H}$ & -0.449316 & 4.295368 & 0.178337 \\
\hline $\mathrm{O}$ & 1.174007 & 0.455649 & 1.566336 \\
\hline 0 & 3.838694 & -0.660483 & 1.305473 \\
\hline 0 & 3.574153 & -0.875653 & -1.484641 \\
\hline 0 & 3.539797 & 2.034021 & 1.132494 \\
\hline $\mathrm{O}$ & 2.009284 & 3.106323 & 2.406964 \\
\hline 0 & -0.369212 & 4.320336 & 1.19677 \\
\hline 0 & -2.090361 & 2.88883 & 2.581866 \\
\hline $\mathrm{O}$ & -2.577089 & 2.427261 & -2.212405 \\
\hline 0 & -3.590783 & 1.37532 & 1.824946 \\
\hline $\mathrm{O}$ & -3.873675 & 1.145345 & -0.871715 \\
\hline $\mathrm{O}$ & -3.314309 & -1.377735 & 2.008377 \\
\hline $\mathrm{O}$ & -3.555859 & -1.628746 & -0.691746 \\
\hline $\mathrm{O}$ & -1.982986 & -2.77871 & -1.838989 \\
\hline
\end{tabular}




\begin{tabular}{|c|c|c|c|}
\hline 0 & -1.54142 & -2.374214 & 3.000062 \\
\hline 0 & -0.963664 & -1.415529 & 0.396484 \\
\hline 0 & -1.183345 & 0.253673 & 2.387958 \\
\hline 0 & 0.199515 & 1.893358 & 4.002067 \\
\hline 0 & 0.578776 & -0.724058 & 4.21672 \\
\hline 0 & -0.433867 & 1.298438 & -3.921218 \\
\hline 0 & -1.223795 & 1.630165 & 0.248712 \\
\hline 0 & 1.140864 & 2.439512 & -0.066093 \\
\hline 0 & 1.497897 & 2.630906 & -2.82329 \\
\hline 0 & -0.572663 & 4.011912 & -1.42355 \\
\hline 0 & 3.272192 & 1.853554 & -1.655009 \\
\hline 0 & 0.683063 & -3.563343 & 1.676608 \\
\hline 0 & 0.160823 & -3.870565 & -0.897859 \\
\hline 0 & 1.547466 & -1.665501 & 0.177547 \\
\hline 0 & 2.523673 & -1.771512 & 2.772754 \\
\hline 0 & 2.030405 & -2.207784 & -2.464721 \\
\hline 0 & -0.402801 & -1.322817 & -3.607092 \\
\hline $\mathrm{O}$ & -1.536823 & -0.025763 & -1.701894 \\
\hline 0 & 0.957388 & 0.285264 & -1.453789 \\
\hline $\mathrm{Zr}$ & -2.482228 & 0.002927 & 0.4203 \\
\hline $\mathrm{Zr}$ & 0.001547 & 2.145782 & 1.920631 \\
\hline $\mathrm{Zr}$ & 0.322014 & -1.423487 & 2.006322 \\
\hline $\mathrm{Zr}$ & 0.043958 & -1.546492 & -1.460228 \\
\hline $\mathrm{Zr}$ & 2.468282 & 0.459908 & -0.069231 \\
\hline $\mathrm{Zr}$ & -0.41485 & 1.914173 & -1.642722 \\
\hline C & 3.784015 & -4.715298 & 0.066767 \\
\hline $\mathrm{H}$ & 3.515896 & -5.610895 & 0.645795 \\
\hline $\mathrm{H}$ & 3.575753 & -4.967672 & -0.984673 \\
\hline $\mathrm{Zn}$ & 2.484221 & -3.328421 & 0.476062 \\
\hline $\mathrm{Zn}$ & -2.450051 & -0.588902 & -3.288698 \\
\hline$C$ & -3.934291 & -0.829158 & -4.514176 \\
\hline $\mathrm{H}$ & -4.109742 & 0.119959 & -5.039947 \\
\hline $\mathrm{H}$ & -4.838517 & -1.006305 & -3.912547 \\
\hline C & 5.263474 & -4.397255 & 0.241008 \\
\hline $\mathrm{H}$ & 5.541413 & -3.442015 & -0.224831 \\
\hline $\mathrm{H}$ & 5.544319 & -4.326881 & 1.299579 \\
\hline $\mathrm{H}$ & 5.90382 & -5.169749 & -0.212519 \\
\hline$C$ & -3.729584 & -1.963494 & -5.511231 \\
\hline $\mathrm{H}$ & -4.589229 & -2.087453 & -6.187316 \\
\hline $\mathrm{H}$ & -2.847744 & -1.798473 & -6.145401 \\
\hline $\mathrm{H}$ & -3.582432 & -2.929053 & -5.007288 \\
\hline
\end{tabular}


Table S22. Structure for $\mathrm{H}_{2} \mathrm{O}, \mathrm{OH}$.

$E=-5084.98966538$ Hartrees, $G=-5084.352097$ Hartrees

\begin{tabular}{|c|c|c|c|}
\hline Atom & $X$ & $\mathrm{Y}$ & Z \\
\hline$C$ & 5.665409 & 2.928341 & 2.550247 \\
\hline$C$ & -5.705596 & 2.910737 & -2.519637 \\
\hline$C$ & 5.663351 & 2.889825 & -2.587823 \\
\hline$C$ & -5.673272 & 2.996148 & 2.443035 \\
\hline C & 4.326205 & 3.176844 & 2.88558 \\
\hline C & -4.362058 & 3.193429 & -2.808005 \\
\hline C & 4.323518 & 3.152146 & -2.911001 \\
\hline$C$ & -4.335748 & 3.276177 & 2.760075 \\
\hline $\mathrm{C}$ & 4.025851 & 4.256293 & 3.73003 \\
\hline C & -4.06143 & 4.212825 & -3.723588 \\
\hline C & 4.034417 & 4.18229 & -3.818355 \\
\hline C & -4.052299 & 4.320938 & 3.651796 \\
\hline C & 5.041216 & 5.077711 & 4.214402 \\
\hline C & -5.081743 & 4.95325 & -4.312845 \\
\hline C & 5.057573 & 4.953388 & -4.363085 \\
\hline C & -5.081975 & 5.087339 & 4.190766 \\
\hline$C$ & 6.388489 & 4.838819 & 3.879331 \\
\hline C & -6.432681 & 4.700655 & -4.004255 \\
\hline C & 6.404118 & 4.715627 & -4.023816 \\
\hline$C$ & -6.42692 & 4.829263 & 3.859768 \\
\hline$C$ & 6.679097 & 3.74348 & 3.043463 \\
\hline C & -6.723399 & 3.651097 & -3.11265 \\
\hline C & 6.685199 & 3.656297 & -3.139606 \\
\hline$C$ & -6.7015 & 3.75745 & 2.989407 \\
\hline$C$ & -5.655075 & -2.743804 & -2.441271 \\
\hline C & 5.677229 & -2.592785 & 2.621475 \\
\hline$C$ & -5.69676 & -2.663177 & 2.530578 \\
\hline$C$ & 5.691274 & -2.628844 & -2.540819 \\
\hline$C$ & -4.316637 & -3.021064 & -2.756865 \\
\hline$C$ & 4.33903 & -2.866574 & 2.94298 \\
\hline C & -4.35245 & -2.943697 & 2.817465 \\
\hline$C$ & 4.353561 & -2.88782 & -2.877789 \\
\hline$C$ & -4.027823 & -4.064365 & -3.648187 \\
\hline$C$ & 4.057894 & -3.896136 & 3.85417 \\
\hline $\mathrm{C}$ & -4.048339 & -3.96119 & 3.733822 \\
\hline$C$ & 4.064566 & -3.967048 & -3.726745 \\
\hline $\mathrm{C}$ & -5.053608 & -4.834349 & -4.187877 \\
\hline $\mathrm{C}$ & 5.088128 & -4.65741 & 4.399492 \\
\hline$C$ & -5.066455 & -4.702841 & 4.325263 \\
\hline
\end{tabular}




$\begin{array}{lrrr}\mathrm{C} & 5.087654 & -4.779367 & -4.210311 \\ \mathrm{C} & -6.399095 & -4.581441 & -3.85692 \\ \mathrm{C} & 6.433144 & -4.410423 & 4.060058 \\ \mathrm{C} & -6.418033 & -4.452807 & 4.01765 \\ \mathrm{C} & 6.432505 & -4.533275 & -3.870504 \\ \mathrm{C} & -6.679472 & -3.509789 & -2.988318 \\ \mathrm{C} & 6.706106 & -3.348697 & 3.175552 \\ \mathrm{C} & -6.712633 & -3.405447 & 3.12487 \\ \mathrm{C} & 6.712665 & -3.435794 & -3.03366 \\ \mathrm{C} & 3.240968 & 2.355818 & -2.262171 \\ \mathrm{C} & 3.243574 & 2.343585 & 2.292423 \\ \mathrm{C} & 3.250508 & -2.081885 & 2.286737 \\ \mathrm{C} & -3.259437 & -2.188579 & 2.146769 \\ \mathrm{C} & -3.229013 & -2.210515 & -2.136513 \\ \mathrm{C} & -3.266496 & 2.439785 & -2.139198 \\ \mathrm{C} & 3.262458 & -2.067958 & -2.278031 \\ \mathrm{C} & -3.24455 & 2.46554 & 2.144646 \\ \mathrm{H} & 5.891814 & 2.094563 & 1.889806 \\ \mathrm{H} & -5.931349 & 2.111386 & -1.817387 \\ \mathrm{H} & 5.883745 & 2.086671 & -1.888736 \\ \mathrm{H} & -5.885992 & 2.177259 & 1.759891 \\ \mathrm{H} & 2.985631 & 4.454419 & 3.978564 \\ \mathrm{H} & -3.019646 & 4.415029 & -3.960744 \\ \mathrm{H} & 2.99482 & 4.387123 & -4.06534 \\ \mathrm{H} & -3.016031 & 4.520899 & 3.915846 \\ \mathrm{H} & 4.786749 & 5.91785 & 4.858794 \\ \mathrm{H} & -4.82977 & 5.746247 & -5.015577 \\ \mathrm{H} & 4.809396 & 5.759461 & -5.051954 \\ \mathrm{H} & -4.842072 & 5.898389 & 4.876788 \\ \mathrm{H} & 7.712686 & 3.535036 & 2.770581 \\ \mathrm{H} & -7.760359 & 3.422752 & -2.870402 \\ \mathrm{H} & 7.718599 & 3.441325 & -2.870694 \\ \mathrm{H} & -7.733619 & 3.527701 & 2.728344 \\ \mathrm{H} & -5.871917 & -1.927662 & -1.756254 \\ \mathrm{H} & 5.891595 & -1.788166 & 1.921942 \\ \mathrm{H} & -5.925885 & -1.867181 & 1.825481 \\ \mathrm{H} & 5.909404 & -1.794799 & -1.877982 \\ \mathrm{H} & -2.987308 & -4.265306 & -3.89533 \\ \mathrm{H} & 3.019585 & -4.108355 & 4.101534 \\ \mathrm{H} & -3.003715 & -4.167323 & 3.955582 \\ \mathrm{H} & 3.030147 & -4.164224 & -4.00172 \\ \mathrm{H} & -4.810217 & -5.647107 & -4.870745 \\ & 4.846141 & -5.463945 & 5.090029\end{array}$




$\begin{array}{rrrr}\mathrm{H} & -4.811704 & -5.497138 & 5.025531 \\ \mathrm{H} & 4.841379 & -5.616858 & -4.861289 \\ \mathrm{H} & -7.712999 & -3.285382 & -2.727815 \\ \mathrm{H} & 7.737893 & -3.122713 & 2.909497 \\ \mathrm{H} & -7.75068 & -3.179824 & 2.884038 \\ \mathrm{H} & 7.743924 & -3.217935 & -2.75995 \\ \mathrm{H} & 7.202771 & 5.322619 & -4.443186 \\ \mathrm{H} & -7.228671 & 5.28605 & -4.45815 \\ \mathrm{H} & 7.236281 & -5.010112 & 4.481316 \\ \mathrm{H} & 7.229733 & -5.169437 & -4.246694 \\ \mathrm{H} & -7.212611 & -5.039964 & 4.47214 \\ \mathrm{H} & -7.23047 & 5.430312 & 4.278549 \\ \mathrm{H} & -7.200253 & -5.186809 & -4.274394 \\ \mathrm{H} & 7.180725 & 5.481472 & 4.255643 \\ \mathrm{H} & -2.004964 & 0.1422 & -2.829579 \\ \mathrm{H} & 1.953525 & 2.992351 & 0.031344 \\ \mathrm{H} & 0.953532 & -1.42003 & 4.326253 \\ \mathrm{H} & 0.017106 & -0.153759 & 4.097949 \\ \mathrm{H} & 0.729816 & -1.519942 & -4.451502 \\ \mathrm{H} & 1.030814 & -4.122024 & -2.163842 \\ \mathrm{H} & -1.999567 & 0.105487 & 2.831791 \\ \mathrm{H} & 2.15205 & -2.613046 & -0.005359 \\ \mathrm{H} & 0.744448 & 1.84742 & 4.380027 \\ \mathrm{H} & 0.014481 & 0.503739 & -4.09248 \\ \mathrm{H} & 0.951743 & 1.776157 & -4.236323 \\ \mathrm{H} & -0.950271 & 4.367832 & 1.606477 \\ \mathrm{H} & -0.767025 & 4.502956 & -1.676667 \\ \mathrm{H} & -0.025749 & 4.192306 & 0.327613 \\ \mathrm{O} & 1.051914 & 0.077423 & 1.477049 \\ \mathrm{O} & 3.592601 & -1.316682 & 1.327796 \\ \mathrm{O} & 3.595819 & -1.215664 & -1.405849 \\ \mathrm{O} & 3.595598 & 1.463658 & 1.444658 \\ \mathrm{O} & 2.048515 & 2.581944 & 2.650897 \\ \mathrm{O} & -0.050468 & 4.109228 & 1.342646 \\ \mathrm{O} & -2.044088 & 2.79318 & 2.404438 \\ \mathrm{O} & -2.07005 & 2.772675 & -2.419051 \\ \mathrm{O} & -3.596831 & 1.496317 & 1.401911 \\ \mathrm{O} & -3.606441 & 1.52054 & -1.332973 \\ \mathrm{O} & -3.608488 & -1.312658 & 1.289905 \\ \mathrm{O} & -3.595019 & -1.249474 & -1.386156 \\ \mathrm{O} & -2.031192 & -2.51015 & -2.41258 \\ \mathrm{O} & -2.077423 & -2.44263 & 2.505469 \\ \mathrm{O} & -1.032419 & -1.450639 & -0.034134\end{array}$




$\begin{array}{lrrr}\mathrm{O} & -1.390835 & 0.086415 & 2.078876 \\ \mathrm{O} & -0.026431 & 1.4589 & 3.941515 \\ \mathrm{O} & 0.058869 & -1.172626 & 4.038266 \\ \mathrm{O} & 0.04885 & 1.516066 & -3.985751 \\ \mathrm{O} & -1.060235 & 1.592841 & 0.035927 \\ \mathrm{O} & 1.385058 & 2.208704 & 0.047545 \\ \mathrm{O} & 2.044816 & 2.565502 & -2.633309 \\ \mathrm{O} & 0.011389 & 4.063358 & -1.304383 \\ \mathrm{O} & 3.599093 & 1.537718 & -1.357279 \\ \mathrm{O} & 0.023651 & -3.820796 & 1.657922 \\ \mathrm{O} & 0.115693 & -3.921729 & -1.901968 \\ \mathrm{O} & 1.380262 & -2.029539 & -0.056302 \\ \mathrm{O} & 2.068143 & -2.247883 & 2.708321 \\ \mathrm{O} & 2.075958 & -2.305156 & -2.676052 \\ \mathrm{O} & -0.017205 & -1.123616 & -3.980564 \\ \mathrm{O} & -1.38976 & 0.164027 & -2.08201 \\ \mathrm{O} & 1.058177 & 0.163039 & -1.488306 \\ \mathrm{Zr} & -2.483386 & 0.088533 & 0.008059 \\ \mathrm{Zr} & 0.018368 & 1.845461 & 1.873573 \\ \mathrm{Zr} & -0.026022 & -1.790664 & 1.82176 \\ \mathrm{Zr} & 0.014684 & -1.584165 & -1.934589 \\ \mathrm{Zr} & 2.479178 & 0.142715 & 0.013464 \\ \mathrm{Zr} & -0.036778 & 2.001438 & -1.721917 \\ \mathrm{Zn} & -0.060562 & -4.08057 & -0.078303\end{array}$


Table S23. Structure for $\mathrm{H}_{2} \mathrm{O}, \mu \mathrm{OH}$.

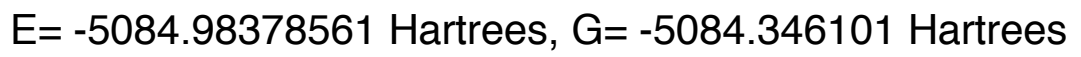

\begin{tabular}{lrrr}
\hline Atom & $\mathrm{X}$ & $\mathrm{Y}$ & \multicolumn{1}{c}{$\mathrm{Z}$} \\
\hline \hline $\mathrm{C}$ & 5.766535 & 2.818299 & 2.489505 \\
$\mathrm{C}$ & -5.610568 & 2.955937 & -2.56483 \\
$\mathrm{C}$ & 5.754383 & 2.657008 & -2.646171 \\
$\mathrm{C}$ & -5.567332 & 3.159161 & 2.394308 \\
$\mathrm{C}$ & 4.434373 & 3.107205 & 2.820406 \\
$\mathrm{C}$ & -4.261075 & 3.198928 & -2.861486 \\
$\mathrm{C}$ & 4.42075 & 2.944018 & -2.973912 \\
$\mathrm{C}$ & -4.222836 & 3.414084 & 2.702966 \\
$\mathrm{C}$ & 4.161801 & 4.213536 & 3.639132 \\
$\mathrm{C}$ & -3.937427 & 4.188507 & -3.801596 \\
$\mathrm{C}$ & 4.155112 & 3.958913 & -3.905329 \\
$\mathrm{C}$ & -3.912528 & 4.472647 & 3.569086 \\
$\mathrm{C}$ & 5.197667 & 5.021375 & 4.102474 \\
$\mathrm{C}$ & -4.940516 & 4.939213 & -4.407192 \\
$\mathrm{C}$ & 5.195711 & 4.691665 & -4.469598 \\
$\mathrm{C}$ & -4.922334 & 5.276526 & 4.09078 \\
$\mathrm{C}$ & 6.538144 & 4.741866 & 3.771592 \\
$\mathrm{C}$ & -6.296634 & 4.726986 & -4.091017 \\
$\mathrm{C}$ & 6.53669 & 4.429421 & -4.126347 \\
$\mathrm{C}$ & -6.273734 & 5.043391 & 3.767661 \\
$\mathrm{C}$ & 6.800587 & 3.620119 & 2.961837 \\
$\mathrm{C}$ & -6.611154 & 3.706427 & -3.174183 \\
$\mathrm{C}$ & 6.793549 & 3.385012 & -3.217366 \\
$\mathrm{C}$ & -6.575802 & 3.958076 & 2.923533 \\
$\mathrm{C}$ & -5.697182 & -2.694682 & -2.351152 \\
$\mathrm{C}$ & 5.644452 & -2.698228 & 2.692869 \\
$\mathrm{C}$ & -5.728033 & -2.494222 & 2.61737 \\
$\mathrm{C}$ & 5.648411 & -2.858033 & -2.467026 \\
$\mathrm{C}$ & -4.366436 & -3.011948 & -2.661566 \\
$\mathrm{C}$ & 4.300565 & -2.931651 & 3.02245 \\
$\mathrm{C}$ & -4.390448 & -2.800501 & 2.909171 \\
$\mathrm{C}$ & 4.304206 & -3.092407 & -2.796083 \\
$\mathrm{C}$ & -4.104598 & -4.082925 & -3.528026 \\
$\mathrm{C}$ & -4.996157 & -3.931927 & 3.958423 \\
$\mathrm{C}$ & 5.00858 & -4.704803 & 4.520535
\end{tabular}




\begin{tabular}{|c|c|c|c|}
\hline C & -5.144238 & -4.505131 & 4.459601 \\
\hline C & 4.989796 & -5.032519 & -4.083849 \\
\hline C & -6.488114 & -4.546986 & -3.721529 \\
\hline C & 6.358588 & -4.498761 & 4.173681 \\
\hline C & -6.489887 & -4.229704 & 4.14775 \\
\hline C & 6.34083 & -4.811124 & -3.751671 \\
\hline C & -6.740847 & -3.44836 & -2.878529 \\
\hline C & 6.655668 & -3.465423 & 3.263694 \\
\hline C & -6.760568 & -3.197121 & 3.230524 \\
\hline C & 6.649043 & -3.701073 & -2.941698 \\
\hline C & 3.320237 & 2.189914 & -2.304833 \\
\hline C & 3.330713 & 2.286464 & 2.248595 \\
\hline C & 3.230188 & -2.136352 & 2.348885 \\
\hline C & -3.28065 & -2.088182 & 2.219511 \\
\hline C & -3.258271 & -2.213428 & -2.062292 \\
\hline C & -3.182841 & 2.435033 & -2.176081 \\
\hline C & 3.234363 & -2.231918 & -2.214966 \\
\hline C & -3.152645 & 2.562652 & 2.105765 \\
\hline $\mathrm{H}$ & 5.970364 & 1.963235 & 1.849138 \\
\hline $\mathrm{H}$ & -5.855191 & 2.179498 & -1.843578 \\
\hline $\mathrm{H}$ & 5.955611 & 1.864395 & -1.929398 \\
\hline $\mathrm{H}$ & -5.801662 & 2.329774 & 1.731217 \\
\hline $\mathrm{H}$ & 3.127223 & 4.442174 & 3.884332 \\
\hline $\mathrm{H}$ & -2.891528 & 4.359327 & -4.0454 \\
\hline $\mathrm{H}$ & 3.120502 & 4.183042 & -4.155767 \\
\hline $\mathrm{H}$ & -2.871296 & 4.653606 & 3.827292 \\
\hline $\mathrm{H}$ & 4.965101 & 5.882827 & 4.726891 \\
\hline $\mathrm{H}$ & -4.670506 & 5.708891 & -5.128777 \\
\hline $\mathrm{H}$ & 4.966148 & 5.486469 & -5.17789 \\
\hline $\mathrm{H}$ & -4.661448 & 6.097641 & 4.756777 \\
\hline $\mathrm{H}$ & 7.828402 & 3.380228 & 2.692796 \\
\hline $\mathrm{H}$ & -7.652988 & 3.50939 & -2.925374 \\
\hline $\mathrm{H}$ & 7.82196 & 3.151347 & -2.944816 \\
\hline $\mathrm{H}$ & -7.613653 & 3.747898 & 2.668764 \\
\hline $\mathrm{H}$ & -5.894316 & -1.852363 & -1.692164 \\
\hline $\mathrm{H}$ & 5.875881 & -1.91667 & 1.973225 \\
\hline $\mathrm{H}$ & -5.937647 & -1.706557 & 1.897486 \\
\hline $\mathrm{H}$ & 5.887896 & -2.015306 & -1.82295 \\
\hline $\mathrm{H}$ & -3.070685 & -4.314722 & -3.776109 \\
\hline $\mathrm{H}$ & 2.954404 & -4.113806 & 4.213758 \\
\hline $\mathrm{H}$ & -3.072135 & -4.018435 & 4.09552 \\
\hline $\mathrm{H}$ & 2.944561 & -4.367566 & -3.870249 \\
\hline $\mathrm{H}$ & -4.927161 & -5.672239 & -4.714328 \\
\hline
\end{tabular}




$\begin{array}{rrrr}\mathrm{H} & 4.749314 & -5.488157 & 5.231428 \\ \mathrm{H} & -4.908603 & -5.284644 & 5.182695 \\ \mathrm{H} & 4.72169 & -5.881899 & -4.710678 \\ \mathrm{H} & -7.767881 & -3.193183 & -2.621283 \\ \mathrm{H} & 7.691803 & -3.273105 & 2.988322 \\ \mathrm{H} & -7.793128 & -2.950639 & 2.987366 \\ \mathrm{H} & 7.686002 & -3.502506 & -2.673894 \\ \mathrm{H} & 7.349193 & 5.006387 & -4.561368 \\ \mathrm{H} & -7.078975 & 5.320545 & -4.557788 \\ \mathrm{H} & 7.147549 & -5.108039 & 4.607875 \\ \mathrm{H} & 7.121954 & -5.476007 & -4.112335 \\ \mathrm{H} & -7.297109 & -4.785232 & 4.618927 \\ \mathrm{H} & -7.061675 & 5.67375 & 4.172698 \\ \mathrm{H} & -7.304486 & -5.141416 & -4.124648 \\ \mathrm{H} & 7.346506 & 5.373875 & 4.13144 \\ \mathrm{H} & -1.925553 & 0.091234 & -2.822357 \\ \mathrm{H} & 2.081427 & 2.912664 & -0.022474 \\ \mathrm{H} & 1.010615 & -1.367241 & 4.286647 \\ \mathrm{H} & 0.077105 & -0.105768 & 4.070938 \\ \mathrm{H} & 0.615306 & -1.707714 & -4.451125 \\ \mathrm{H} & 0.945762 & -4.114478 & -1.347328 \\ \mathrm{H} & -1.033558 & -4.108253 & 2.135491 \\ \mathrm{H} & -1.878594 & 0.080351 & 2.833077 \\ \mathrm{H} & 0.814352 & 1.9052 & 4.352968 \\ \mathrm{H} & 0.057038 & 0.382476 & -4.084718 \\ \mathrm{H} & 1.033911 & 1.619744 & -4.257495 \\ \mathrm{H} & -0.819973 & 4.458252 & 1.504681 \\ \mathrm{H} & -0.668202 & 4.474667 & -1.759643 \\ \mathrm{H} & 0.103451 & 4.210983 & 0.234691 \\ \mathrm{O} & 1.137514 & 0.142851 & 1.575915 \\ \mathrm{O} & 3.600507 & -1.308776 & 1.472206 \\ \mathrm{O} & 3.60276 & -1.37847 & -1.353224 \\ \mathrm{O} & 3.651631 & 1.393027 & 1.411604 \\ \mathrm{O} & 2.14331 & 2.583355 & 2.609692 \\ \mathrm{O} & 0.069989 & 4.15832 & 1.254087 \\ \mathrm{O} & -1.946727 & 2.879029 & 2.340043 \\ \mathrm{O} & -1.982194 & 2.724319 & -2.468833 \\ \mathrm{O} & -3.532195 & 1.578707 & 1.395001 \\ \mathrm{O} & -3.550759 & 1.560205 & -1.331486 \\ \mathrm{O} & -3.621818 & -1.216785 & 1.350333 \\ \mathrm{O} & -3.586351 & -1.156967 & -1.449185 \\ \mathrm{O} & -2.076434 & -2.694026 & -2.153051 \\ \mathrm{O} & -2.091803 & -2.378057 & 2.542327\end{array}$




$\begin{array}{lrrr}\mathrm{O} & -1.188149 & -1.526902 & -0.006189 \\ \mathrm{O} & -1.318242 & 0.132796 & 2.044867 \\ \mathrm{O} & 0.02206 & 1.552729 & 3.921247 \\ \mathrm{O} & 0.115675 & -1.117955 & 4.003205 \\ \mathrm{O} & 0.119445 & 1.398852 & -4.009928 \\ \mathrm{O} & -0.974517 & 1.57097 & 0.017887 \\ \mathrm{O} & 1.457978 & 2.173007 & 0.012703 \\ \mathrm{O} & 2.129676 & 2.461005 & -2.668749 \\ \mathrm{O} & 0.106702 & 4.035653 & -1.379921 \\ \mathrm{O} & 3.653563 & 1.371722 & -1.396078 \\ \mathrm{O} & -0.353356 & -3.936462 & 1.463421 \\ \mathrm{O} & 0.078283 & -3.854351 & -1.698951 \\ \mathrm{O} & 1.293452 & -1.905057 & 0.023685 \\ \mathrm{O} & 2.021849 & -2.358318 & 2.699276 \\ \mathrm{O} & 2.057773 & -2.386159 & -2.653542 \\ \mathrm{O} & -0.04981 & -1.238284 & -3.928468 \\ \mathrm{O} & -1.341154 & 0.126208 & -2.051071 \\ \mathrm{O} & 1.13384 & 0.152775 & -1.536098 \\ \mathrm{Zr} & -2.468093 & 0.160639 & 0.014175 \\ \mathrm{Zr} & 0.106152 & 1.920658 & 1.856527 \\ \mathrm{Zr} & 0.1043 & -1.652843 & 1.707116 \\ \mathrm{Zr} & 0.111889 & -1.604126 & -1.861226 \\ \mathrm{Zr} & 2.507557 & -0.003701 & 0.010572 \\ \mathrm{Zr} & 0.05831 & 1.964007 & -1.76303 \\ \mathrm{Zn} & -1.135014 & -3.596199 & -0.248294\end{array}$


Table S24. Structure for 4-coordinated $\mathrm{Zn}$ with one water. $E=-5161.43528515$ Hartrees, $G=-5160.80185$ Hartrees

\begin{tabular}{|c|c|c|c|}
\hline Atom & $\mathrm{X}$ & $\mathrm{Y}$ & Z \\
\hline C & 5.774873 & 2.795835 & 2.567209 \\
\hline $\mathrm{C}$ & -5.592086 & 3.138634 & -2.500194 \\
\hline $\mathrm{C}$ & 5.771683 & 2.795378 & -2.571012 \\
\hline $\mathrm{C}$ & -5.557312 & 3.186395 & 2.462966 \\
\hline C & 4.44327 & 3.079769 & 2.904672 \\
\hline $\mathrm{C}$ & -4.241061 & 3.385184 & -2.786758 \\
\hline C & 4.439839 & 3.098014 & -2.891957 \\
\hline $\mathrm{C}$ & -4.212367 & 3.425966 & 2.7818 \\
\hline C & 4.173696 & 4.161052 & 3.757129 \\
\hline C & -3.911613 & 4.402372 & -3.694847 \\
\hline $\mathrm{C}$ & 4.180102 & 4.142643 & -3.791603 \\
\hline C & -3.89936 & 4.455653 & 3.681124 \\
\hline C & 5.211982 & 4.949702 & 4.247338 \\
\hline C & -4.910492 & 5.175804 & -4.278401 \\
\hline C & 5.224738 & 4.888383 & -4.330841 \\
\hline C & -4.906853 & 5.246974 & 4.225973 \\
\hline C & 6.551925 & 4.675133 & 3.910213 \\
\hline C & -6.268056 & 4.959399 & -3.971391 \\
\hline C & 6.56399 & 4.609979 & -3.993629 \\
\hline C & -6.258588 & 5.029653 & 3.893365 \\
\hline C & 6.811302 & 3.578195 & 3.066206 \\
\hline C & -6.588457 & 3.91196 & -3.087502 \\
\hline C & 6.81488 & 3.536586 & -3.117333 \\
\hline C & -6.563501 & 3.972535 & 3.015163 \\
\hline C & -5.702161 & -2.515524 & -2.463592 \\
\hline C & 5.629896 & -2.723788 & 2.597616 \\
\hline C & -5.741504 & -2.470517 & 2.508642 \\
\hline C & 5.642868 & -2.722078 & -2.564765 \\
\hline C & -4.372152 & -2.828344 & -2.78149 \\
\hline C & 4.284474 & -2.961857 & 2.917331 \\
\hline C & -4.405736 & -2.791203 & 2.7931 \\
\hline C & 4.298341 & -2.940477 & -2.903325 \\
\hline C & -4.113078 & -3.872774 & -3.680622 \\
\hline C & 3.97421 & -3.989681 & 3.821024 \\
\hline C & -4.130596 & -3.823646 & 3.701932 \\
\hline C & 3.978806 & -4.004719 & -3.760221 \\
\hline C & -5.160319 & -4.609312 & -4.225756 \\
\hline C & 4.982414 & -4.783932 & 4.36046 \\
\hline C & -5.169365 & -4.540428 & 4.288108 \\
\hline
\end{tabular}




\begin{tabular}{|c|c|c|c|}
\hline C & 4.978414 & -4.842174 & -4.249987 \\
\hline C & -6.498081 & -4.320735 & -3.892667 \\
\hline C & 6.3339 & -4.572729 & 4.022578 \\
\hline C & -6.5133 & -4.249833 & 3.98266 \\
\hline C & 6.329714 & -4.636877 & -3.908682 \\
\hline C & -6.747905 & -3.24799 & -3.016125 \\
\hline C & 6.636898 & -3.512662 & 3.145896 \\
\hline C & -6.778045 & -3.187949 & 3.097713 \\
\hline C & 6.640938 & -3.553999 & -3.063826 \\
\hline C & 3.335106 & 2.328005 & -2.248789 \\
\hline C & 3.337426 & 2.282043 & 2.305569 \\
\hline C & 3.218725 & -2.141758 & 2.267364 \\
\hline C & -3.291744 & -2.062305 & 2.128264 \\
\hline C & -3.261974 & -2.053503 & -2.155788 \\
\hline C & -3.167355 & 2.595776 & -2.123796 \\
\hline C & 3.231076 & -2.094289 & -2.297517 \\
\hline C & -3.14469 & 2.589223 & 2.160111 \\
\hline $\mathrm{H}$ & 5.97751 & 1.960681 & 1.900851 \\
\hline $\mathrm{H}$ & -5.840342 & 2.341345 & -1.803188 \\
\hline $\mathrm{H}$ & 5.969328 & 1.980372 & -1.878922 \\
\hline $\mathrm{H}$ & -5.792931 & 2.379708 & 1.772805 \\
\hline $\mathrm{H}$ & 3.139505 & 4.38673 & 4.00739 \\
\hline $\mathrm{H}$ & -2.864453 & 4.576899 & -3.93037 \\
\hline $\mathrm{H}$ & 3.146729 & 4.378515 & -4.037057 \\
\hline $\mathrm{H}$ & -2.857722 & 4.624287 & 3.94596 \\
\hline $\mathrm{H}$ & 4.981542 & 5.791944 & 4.898013 \\
\hline $\mathrm{H}$ & -4.636048 & 5.966597 & -4.975131 \\
\hline $\mathrm{H}$ & 4.999581 & 5.706045 & -5.013956 \\
\hline $\mathrm{H}$ & -4.643994 & 6.046082 & 4.917548 \\
\hline $\mathrm{H}$ & 7.83851 & 3.34229 & 2.791681 \\
\hline $\mathrm{H}$ & -7.631525 & 3.711745 & -2.846497 \\
\hline $\mathrm{H}$ & 7.841704 & 3.289845 & -2.850659 \\
\hline $\mathrm{H}$ & -7.601778 & 3.774604 & 2.752312 \\
\hline $\mathrm{H}$ & -5.895174 & -1.69858 & -1.772377 \\
\hline $\mathrm{H}$ & 5.86703 & -1.922145 & 1.902109 \\
\hline $\mathrm{H}$ & -5.947324 & -1.662129 & 1.810589 \\
\hline $\mathrm{H}$ & 5.885589 & -1.900666 & -1.894743 \\
\hline $\mathrm{H}$ & -3.082114 & -4.098856 & -3.944152 \\
\hline $\mathrm{H}$ & 2.929975 & -4.175392 & 4.065007 \\
\hline $\mathrm{H}$ & -3.092911 & -4.054159 & 3.934999 \\
\hline $\mathrm{H}$ & 2.934325 & -4.178602 & -4.011532 \\
\hline $\mathrm{H}$ & -4.940244 & -5.422368 & -4.915925 \\
\hline $\mathrm{H}$ & 4.717687 & -5.589336 & 5.04405 \\
\hline
\end{tabular}




\begin{tabular}{|c|c|c|c|}
\hline $\mathrm{H}$ & -4.937295 & -5.343988 & 4.985469 \\
\hline $\mathrm{H}$ & 4.707699 & -5.671055 & -4.902487 \\
\hline $\mathrm{H}$ & -7.77462 & -2.995255 & -2.755071 \\
\hline $\mathrm{H}$ & 7.674634 & -3.314754 & 2.880511 \\
\hline $\mathrm{H}$ & -7.809061 & -2.930824 & 2.859079 \\
\hline $\mathrm{H}$ & 7.67809 & -3.368251 & -2.787935 \\
\hline $\mathrm{H}$ & 7.379563 & 5.196976 & -4.408898 \\
\hline $\mathrm{H}$ & -7.047099 & 5.5706 & -4.420624 \\
\hline $\mathrm{H}$ & 7.119643 & -5.198647 & 4.438543 \\
\hline $\mathrm{H}$ & 7.108841 & -5.29338 & -4.288392 \\
\hline $\mathrm{H}$ & -7.323911 & -4.817063 & 4.433745 \\
\hline $\mathrm{H}$ & -7.04472 & 5.650362 & 4.3165 \\
\hline $\mathrm{H}$ & -7.315743 & -4.899768 & -4.315108 \\
\hline $\mathrm{H}$ & 7.362092 & 5.292234 & 4.291079 \\
\hline $\mathrm{H}$ & -1.948591 & 0.258813 & -2.824735 \\
\hline $\mathrm{H}$ & 2.067191 & 2.990497 & 0.052305 \\
\hline $\mathrm{H}$ & 0.908546 & -1.467303 & 4.332637 \\
\hline $\mathrm{H}$ & 0.031222 & -0.163549 & 4.091529 \\
\hline $\mathrm{H}$ & 0.728667 & -1.470439 & -4.440967 \\
\hline $\mathrm{H}$ & -0.838288 & -4.042603 & -2.376231 \\
\hline $\mathrm{H}$ & -1.956656 & 0.201773 & 2.830655 \\
\hline $\mathrm{H}$ & 2.032076 & -2.695032 & -0.073641 \\
\hline $\mathrm{H}$ & 0.826301 & 1.817388 & 4.3971 \\
\hline $\mathrm{H}$ & 0.056882 & 0.554217 & -4.080059 \\
\hline $\mathrm{H}$ & 1.030342 & 1.799426 & -4.232016 \\
\hline $\mathrm{H}$ & -0.794604 & 4.419209 & 1.642327 \\
\hline $\mathrm{H}$ & -0.615487 & 4.575992 & -1.642735 \\
\hline $\mathrm{H}$ & 0.123302 & 4.230116 & 0.361514 \\
\hline 0 & 1.090527 & 0.082134 & 1.462638 \\
\hline 0 & 3.583457 & -1.385701 & 1.311586 \\
\hline $\mathrm{O}$ & 3.597956 & -1.277088 & -1.396784 \\
\hline 0 & 3.665971 & 1.399267 & 1.452302 \\
\hline 0 & 2.149987 & 2.548372 & 2.670565 \\
\hline 0 & 0.097661 & 4.1393 & 1.375152 \\
\hline $\mathrm{O}$ & -1.93574 & 2.874076 & 2.42551 \\
\hline 0 & -1.961526 & 2.894924 & -2.398513 \\
\hline 0 & -3.52521 & 1.636227 & 1.408038 \\
\hline $\mathrm{O}$ & -3.533127 & 1.674238 & -1.328995 \\
\hline 0 & -3.616451 & -1.161962 & 1.291683 \\
\hline $\mathrm{O}$ & -3.593345 & -1.118055 & -1.363289 \\
\hline $\mathrm{O}$ & -2.075336 & -2.372781 & -2.464532 \\
\hline $\mathrm{O}$ & -2.117428 & -2.402716 & 2.443468 \\
\hline $\mathrm{O}$ & -1.086048 & -1.41431 & -0.042853 \\
\hline
\end{tabular}




$\begin{array}{lrrr}\mathrm{O} & -1.355399 & 0.149003 & 2.073464 \\ \mathrm{O} & 0.046747 & 1.458089 & 3.949183 \\ \mathrm{O} & 0.031405 & -1.181793 & 4.026297 \\ \mathrm{O} & 0.122624 & 1.566245 & -3.972749 \\ \mathrm{O} & -0.97929 & 1.675003 & 0.047372 \\ \mathrm{O} & 1.48225 & 2.21902 & 0.060513 \\ \mathrm{O} & 2.144985 & 2.57474 & -2.618736 \\ \mathrm{O} & 0.155145 & 4.117886 & -1.276469 \\ \mathrm{O} & 3.671992 & 1.491792 & -1.353626 \\ \mathrm{O} & 0.037644 & -3.767837 & 1.650046 \\ \mathrm{O} & -0.012789 & -3.833332 & -1.90461 \\ \mathrm{O} & 1.344824 & -2.012881 & -0.089557 \\ \mathrm{O} & 2.039995 & -2.254141 & 2.709778 \\ \mathrm{O} & 2.048261 & -2.241889 & -2.7247 \\ \mathrm{O} & -0.02641 & -1.072835 & -3.982893 \\ \mathrm{O} & -1.345255 & 0.25837 & -2.067323 \\ \mathrm{O} & 1.100575 & 0.197678 & -1.481333 \\ \mathrm{Zr} & -2.430303 & 0.223305 & 0.011736 \\ \mathrm{Zr} & 0.113753 & 1.866732 & 1.886577 \\ \mathrm{Zr} & -0.011426 & -1.775685 & 1.79849 \\ \mathrm{Zr} & 0.034116 & -1.530485 & -1.933864 \\ \mathrm{Zr} & 2.533432 & 0.107281 & 0.006465 \\ \mathrm{Zr} & 0.062207 & 2.061442 & -1.71149 \\ \mathrm{Zn} & -0.329096 & -4.082222 & -0.084416 \\ \mathrm{O} & -2.507038 & -4.066214 & 0.043539 \\ \mathrm{H} & -2.546519 & -4.178195 & 1.012447 \\ \mathrm{H} & -2.54261 & -3.089525 & -0.057442\end{array}$


Table S25. Structure for 4-coordinated $\mathrm{Zn}$ with two waters. $E=-5237.86342825$ Hartrees, $G=-5237.182235$ Hartrees

\begin{tabular}{|c|c|c|c|}
\hline Atom & $X$ & $\mathrm{Y}$ & Z \\
\hline$C$ & 5.326346 & 3.489824 & 2.515313 \\
\hline$C$ & -5.999097 & 2.527319 & -2.565174 \\
\hline C & 5.338166 & 3.376611 & -2.621574 \\
\hline$C$ & -5.983957 & 2.687069 & 2.395722 \\
\hline$C$ & 3.971331 & 3.639752 & 2.845736 \\
\hline$C$ & -4.680603 & 2.907886 & -2.856226 \\
\hline$C$ & 3.982773 & 3.530909 & -2.94988 \\
\hline$C$ & -4.67249 & 3.073315 & 2.709994 \\
\hline$C$ & 3.587398 & 4.705128 & 3.674018 \\
\hline$C$ & -4.456945 & 3.933983 & -3.786352 \\
\hline$C$ & 3.617653 & 4.522359 & -3.872649 \\
\hline$C$ & -4.471796 & 4.149555 & 3.586615 \\
\hline$C$ & 4.535821 & 5.608954 & 4.147227 \\
\hline$C$ & -5.529719 & 4.585354 & -4.387488 \\
\hline$C$ & 4.57988 & 5.361656 & -4.427513 \\
\hline$C$ & -5.558294 & 4.842563 & 4.113255 \\
\hline$C$ & 5.89814 & 5.469166 & 3.817025 \\
\hline$C$ & -6.857993 & 4.234502 & -4.076585 \\
\hline $\mathrm{C}$ & 5.939955 & 5.232729 & -4.083439 \\
\hline C & -6.878815 & 4.477409 & 3.784726 \\
\hline C & 6.273569 & 4.387287 & 2.997583 \\
\hline C & -7.069355 & 3.178831 & -3.169983 \\
\hline C & 6.299474 & 4.211013 & -3.183517 \\
\hline C & -7.068637 & 3.37518 & 2.929877 \\
\hline C & -5.515745 & -3.105104 & -2.403788 \\
\hline$C$ & 5.760891 & -2.012394 & 2.667668 \\
\hline C & -5.574081 & -2.955428 & 2.566309 \\
\hline C & 5.788653 & -2.121983 & -2.494326 \\
\hline C & -4.159518 & -3.28334 & -2.713947 \\
\hline C & 4.446823 & -2.383395 & 2.991758 \\
\hline C & -4.21284 & -3.128165 & 2.858339 \\
\hline C & 4.474748 & -2.489005 & -2.828316 \\
\hline C & -3.789576 & -4.314392 & -3.589722 \\
\hline C & 4.243532 & -3.418203 & 3.917405 \\
\hline C & -3.83362 & -4.10588 & 3.789907 \\
\hline C & 4.271708 & -3.598849 & -3.661038 \\
\hline C & -4.752215 & -5.168464 & -4.119118 \\
\hline C & 5.327897 & -4.09023 & 4.474922 \\
\hline C & -4.793193 & -4.914677 & 4.391137 \\
\hline
\end{tabular}




\begin{tabular}{|c|c|c|c|}
\hline C & 5.355081 & -4.336805 & -4.132342 \\
\hline C & -6.113828 & -5.014591 & -3.793276 \\
\hline C & 6.650758 & -3.74587 & 4.133263 \\
\hline C & -6.159298 & -4.773408 & 4.078559 \\
\hline C & 6.676393 & -3.983582 & -3.794655 \\
\hline C & -6.477307 & -3.955069 & -2.940748 \\
\hline C & 6.843473 & -2.679289 & 3.233599 \\
\hline C & -6.531363 & -3.764758 & 3.170263 \\
\hline C & 6.869863 & -2.855934 & -2.973554 \\
\hline C & 2.964022 & 2.664142 & -2.290073 \\
\hline C & 2.956519 & 2.717887 & 2.264096 \\
\hline C & 3.303859 & -1.693119 & 2.322137 \\
\hline C & -3.177882 & -2.301797 & 2.177317 \\
\hline C & -3.136574 & -2.38275 & -2.105606 \\
\hline C & -3.531324 & 2.250231 & -2.175714 \\
\hline C & 3.318957 & -1.746444 & -2.240313 \\
\hline C & -3.520858 & 2.339516 & 2.107323 \\
\hline $\mathrm{H}$ & 5.616466 & 2.667844 & 1.865022 \\
\hline $\mathrm{H}$ & -6.16409 & 1.723987 & -1.850636 \\
\hline $\mathrm{H}$ & 5.617652 & 2.603556 & -1.909892 \\
\hline $\mathrm{H}$ & -6.131673 & 1.844163 & 1.724822 \\
\hline $\mathrm{H}$ & 2.534311 & 4.82645 & 3.917726 \\
\hline $\mathrm{H}$ & -3.433107 & 4.211699 & -4.025236 \\
\hline $\mathrm{H}$ & 2.565907 & 4.643116 & -4.123526 \\
\hline $\mathrm{H}$ & -3.454303 & 4.431724 & 3.848626 \\
\hline $\mathrm{H}$ & 4.216581 & 6.436766 & 4.778631 \\
\hline $\mathrm{H}$ & -5.337806 & 5.385028 & -5.101592 \\
\hline $\mathrm{H}$ & 4.272195 & 6.136333 & -5.1283 \\
\hline $\mathrm{H}$ & -5.382851 & 5.679592 & 4.787542 \\
\hline $\mathrm{H}$ & 7.320639 & 4.255665 & 2.727955 \\
\hline $\mathrm{H}$ & -8.086393 & 2.875558 & -2.925262 \\
\hline $\mathrm{H}$ & 7.345817 & 4.080193 & -2.910334 \\
\hline $\mathrm{H}$ & -8.079684 & 3.063234 & 2.67142 \\
\hline $\mathrm{H}$ & -5.794402 & -2.298214 & -1.730251 \\
\hline $\mathrm{H}$ & 5.917364 & -1.195095 & 1.966415 \\
\hline $\mathrm{H}$ & -5.861742 & -2.188687 & 1.850301 \\
\hline $\mathrm{H}$ & 5.945536 & -1.257128 & -1.852998 \\
\hline $\mathrm{H}$ & -2.740091 & -4.433534 & -3.850844 \\
\hline $\mathrm{H}$ & 3.222775 & -3.703203 & 4.165224 \\
\hline $\mathrm{H}$ & -2.77657 & -4.228369 & 4.014475 \\
\hline $\mathrm{H}$ & 3.253564 & -3.872315 & -3.935135 \\
\hline $\mathrm{H}$ & -4.446569 & -5.96847 & -4.791937 \\
\hline $\mathrm{H}$ & 5.146962 & -4.902282 & 5.177234 \\
\hline
\end{tabular}




\begin{tabular}{|c|c|c|c|}
\hline $\mathrm{H}$ & -4.479864 & -5.677078 & 5.103076 \\
\hline $\mathrm{H}$ & 5.175515 & -5.199655 & -4.771926 \\
\hline $\mathrm{H}$ & -7.525575 & -3.80628 & -2.685308 \\
\hline $\mathrm{H}$ & 7.855324 & -2.376755 & 2.967886 \\
\hline $\mathrm{H}$ & -7.583266 & -3.622885 & 2.925317 \\
\hline $\mathrm{H}$ & 7.881226 & -2.554132 & -2.704954 \\
\hline $\mathrm{H}$ & 6.690663 & 5.893151 & -4.510646 \\
\hline $\mathrm{H}$ & -7.695554 & 4.750617 & -4.539706 \\
\hline $\mathrm{H}$ & 7.496659 & -4.275767 & 4.563671 \\
\hline $\mathrm{H}$ & 7.520855 & -4.561593 & -4.161444 \\
\hline $\mathrm{H}$ & -6.907643 & -5.413005 & 4.540799 \\
\hline $\mathrm{H}$ & -7.726991 & 5.021163 & 4.193823 \\
\hline $\mathrm{H}$ & -6.865069 & -5.685459 & -4.203166 \\
\hline $\mathrm{H}$ & 6.637939 & 6.176365 & 4.184271 \\
\hline $\mathrm{H}$ & -2.075188 & 0.047072 & -2.834131 \\
\hline $\mathrm{H}$ & 1.619385 & 3.239999 & -0.002818 \\
\hline $\mathrm{H}$ & 0.933266 & -1.210219 & 4.34877 \\
\hline $\mathrm{H}$ & -0.087135 & -0.025104 & 4.087757 \\
\hline $\mathrm{H}$ & 0.748096 & -1.447477 & -4.399469 \\
\hline $\mathrm{H}$ & -0.654347 & -4.069494 & -2.025677 \\
\hline $\mathrm{H}$ & -2.108311 & 0.116779 & 2.835037 \\
\hline $\mathrm{H}$ & 0.499257 & 2.045147 & 4.363861 \\
\hline $\mathrm{H}$ & -0.101493 & 0.514337 & -4.07632 \\
\hline $\mathrm{H}$ & 0.733468 & 1.85475 & -4.258567 \\
\hline $\mathrm{H}$ & -1.380554 & 4.386525 & 1.549142 \\
\hline $\mathrm{H}$ & -1.200928 & 4.494168 & -1.738238 \\
\hline $\mathrm{H}$ & -0.442295 & 4.269233 & 0.275277 \\
\hline $\mathrm{O}$ & 0.95594 & 0.268433 & 1.480965 \\
\hline $\mathrm{O}$ & 3.604085 & -0.939014 & 1.334889 \\
\hline 0 & 3.604204 & -0.908086 & -1.323908 \\
\hline $\mathrm{O}$ & 3.380524 & 1.854306 & 1.431589 \\
\hline $\mathrm{O}$ & 1.747669 & 2.866324 & 2.619451 \\
\hline 0 & -0.461047 & 4.201861 & 1.290888 \\
\hline $\mathrm{O}$ & -2.35005 & 2.758123 & 2.369067 \\
\hline $\mathrm{O}$ & -2.363073 & 2.667287 & -2.461095 \\
\hline $\mathrm{O}$ & -3.798973 & 1.340112 & 1.374125 \\
\hline $\mathrm{O}$ & -3.80233 & 1.319457 & -1.356939 \\
\hline $\mathrm{O}$ & -3.591053 & -1.463658 & 1.312222 \\
\hline $\mathrm{O}$ & -3.568656 & -1.449152 & -1.365096 \\
\hline $\mathrm{O}$ & -1.923684 & -2.592481 & -2.401597 \\
\hline 0 & -1.981614 & -2.475341 & 2.532441 \\
\hline $\mathrm{O}$ & -1.004479 & -1.387987 & -0.007668 \\
\hline $\mathrm{O}$ & -1.498641 & 0.113251 & 2.082967 \\
\hline
\end{tabular}




$\begin{array}{lrrr}\mathrm{O} & -0.238992 & 1.597067 & 3.926546 \\ \mathrm{O} & 0.026598 & -1.036421 & 4.047549 \\ \mathrm{O} & -0.145593 & 1.532001 & -3.997909 \\ \mathrm{O} & -1.271236 & 1.617129 & 0.017079 \\ \mathrm{O} & 1.122018 & 2.409812 & 0.02291 \\ \mathrm{O} & 1.756369 & 2.77809 & -2.662064 \\ \mathrm{O} & -0.38812 & 4.125634 & -1.36226 \\ \mathrm{O} & 3.385376 & 1.884312 & -1.377382 \\ \mathrm{O} & 0.271123 & -3.672384 & 1.744969 \\ \mathrm{O} & 0.239805 & -3.777466 & -1.781704 \\ \mathrm{O} & 1.457969 & -1.866801 & -0.029102 \\ \mathrm{O} & 2.148979 & -1.921666 & 2.759942 \\ \mathrm{O} & 2.164325 & -2.041562 & -2.655356 \\ \mathrm{O} & -0.027396 & -1.097352 & -3.938148 \\ \mathrm{O} & -1.47975 & 0.12202 & -2.074375 \\ \mathrm{O} & 0.969834 & 0.320687 & -1.483386 \\ \mathrm{Zr} & -2.570013 & 0.00656 & 0.013341 \\ \mathrm{Zr} & -0.217528 & 1.952072 & 1.853908 \\ \mathrm{Zr} & -0.001021 & -1.70889 & 1.830935 \\ \mathrm{Zr} & 0.051968 & -1.531317 & -1.877599 \\ \mathrm{Zr} & 2.355859 & 0.443229 & 0.018795 \\ \mathrm{Zr} & -0.27501 & 2.058692 & -1.746592 \\ \mathrm{Zn} & 0.735464 & -4.11905 & 0.059659 \\ \mathrm{O} & 2.413073 & -5.003759 & -0.788377 \\ \mathrm{H} & 2.207607 & -4.740278 & -1.704885 \\ \mathrm{H} & 3.237649 & -4.51277 & -0.539563 \\ \mathrm{H} & 2.338792 & -2.273536 & 0.019248 \\ \mathrm{O} & 4.440992 & -3.431889 & 0.073072 \\ \mathrm{H} & 4.735002 & -3.587662 & 0.984228 \\ \mathrm{H} & 4.46871 & -2.46043 & -0.025597 \\ & & & \\ & & & \end{array}$


Table S26. Structure for 5-coordinated $\mathrm{Zn}$ with two waters. $E=-5237.87448431$ Hartrees, $G=-5237.192392$ Hartrees

\begin{tabular}{|c|c|c|c|}
\hline Atom & $\mathrm{X}$ & $\mathrm{Y}$ & Z \\
\hline C & 5.931945 & 2.390071 & 2.743915 \\
\hline $\mathrm{C}$ & -5.368832 & 3.60666 & -2.337079 \\
\hline $\mathrm{C}$ & 5.96561 & 2.719895 & -2.383623 \\
\hline $\mathrm{C}$ & -5.367424 & 3.333726 & 2.618938 \\
\hline C & 4.613104 & 2.715787 & 3.093542 \\
\hline $\mathrm{C}$ & -4.005488 & 3.805584 & -2.601607 \\
\hline C & 4.65215 & 3.106562 & -2.689764 \\
\hline $\mathrm{C}$ & -4.014834 & 3.487066 & 2.958041 \\
\hline C & 4.389594 & 3.751808 & 4.012901 \\
\hline C & -3.621148 & 4.861873 & -3.440826 \\
\hline $\mathrm{C}$ & 4.449281 & 4.218157 & -3.521191 \\
\hline C & -3.659291 & 4.440462 & 3.923215 \\
\hline C & 5.460967 & 4.456229 & 4.557199 \\
\hline C & -4.577567 & 5.71856 & -3.977334 \\
\hline C & 5.532327 & 4.945635 & -4.006944 \\
\hline C & -4.63155 & 5.242921 & 4.513861 \\
\hline C & 6.788583 & 4.139456 & 4.208517 \\
\hline C & -5.946117 & 5.548739 & -3.690454 \\
\hline C & 6.854229 & 4.581747 & -3.682946 \\
\hline C & -5.98973 & 5.113001 & 4.162422 \\
\hline C & 7.001099 & 3.087762 & 3.296558 \\
\hline C & -6.322723 & 4.463385 & -2.877299 \\
\hline C & 7.047051 & 3.443403 & -2.876716 \\
\hline C & -6.338685 & 4.130465 & 3.21661 \\
\hline C & -5.750238 & -2.026282 & -2.665714 \\
\hline C & 5.522168 & -3.10668 & 2.417453 \\
\hline C & -5.823059 & -2.298978 & 2.298703 \\
\hline C & 5.57225 & -2.773937 & -2.733812 \\
\hline C & -4.434581 & -2.381762 & -2.997787 \\
\hline C & 4.164684 & -3.299385 & 2.715725 \\
\hline C & -4.506304 & -2.701326 & 2.567334 \\
\hline C & 4.221351 & -2.904761 & -3.091273 \\
\hline C & -4.219391 & -3.377647 & -3.961399 \\
\hline C & 3.798944 & -4.366994 & 3.549978 \\
\hline C & -4.287592 & -3.802113 & 3.40885 \\
\hline C & 3.857222 & -3.895093 & -4.016416 \\
\hline C & -5.296811 & -4.0262 & -4.557145 \\
\hline C & 4.763991 & -5.242076 & 4.041119 \\
\hline C & -5.363607 & -4.504183 & 3.943305 \\
\hline
\end{tabular}




\begin{tabular}{lrrr}
$\mathrm{C}$ & 4.818993 & -4.746715 & -4.55508 \\
$\mathrm{C}$ & -6.62155 & -3.695296 & -4.211592 \\
$\mathrm{C}$ & 6.126443 & -5.075152 & 3.723083 \\
$\mathrm{C}$ & -6.689865 & -4.129856 & 3.651776 \\
$\mathrm{C}$ & 6.176102 & -4.629312 & -4.195742 \\
$\mathrm{C}$ & -6.825915 & -2.670266 & -3.26869 \\
$\mathrm{C}$ & 6.486222 & -3.976827 & 2.917875 \\
$\mathrm{C}$ & -6.89701 & -3.00176 & 2.836136 \\
$\mathrm{C}$ & 6.532836 & -3.619263 & -3.281519 \\
$\mathrm{C}$ & 3.507107 & 2.351088 & -2.10207 \\
$\mathrm{C}$ & 3.474535 & 2.012594 & 2.439682 \\
$\mathrm{C}$ & 3.144312 & -2.388715 & 2.116184 \\
$\mathrm{C}$ & -3.354063 & -1.986073 & 1.956129 \\
$\mathrm{C}$ & -3.29295 & -1.703494 & -2.319496 \\
$\mathrm{C}$ & -2.975659 & 2.924273 & -1.986477 \\
$\mathrm{C}$ & 3.191553 & -2.048874 & -2.43706 \\
$\mathrm{C}$ & -2.984108 & 2.641425 & 2.287972 \\
$\mathrm{H}$ & 6.098812 & 1.590609 & 2.025922 \\
$\mathrm{H}$ & -5.660049 & 2.778846 & -1.694479 \\
$\mathrm{H}$ & 6.118933 & 1.853548 & -1.744777 \\
$\mathrm{H}$ & -5.636661 & 2.585075 & 1.877694 \\
$\mathrm{H}$ & 3.365601 & 4.010671 & 4.272725 \\
$\mathrm{H}$ & -2.565115 & 5.000314 & -3.660241 \\
$\mathrm{H}$ & 3.430099 & 4.518644 & -3.755182 \\
$\mathrm{H}$ & -2.612585 & 4.540841 & 4.20242 \\
$\mathrm{H}$ & 5.26669 & 5.265074 & 5.260025 \\
$\mathrm{H}$ & -4.260593 & 6.538363 & -4.620433 \\
$\mathrm{H}$ & 5.351693 & 5.815499 & -4.636763 \\
$\mathrm{H}$ & -4.335642 & 5.982181 & 5.256724 \\
$\mathrm{H}$ & 8.017821 & 2.820721 & 3.01161 \\
$\mathrm{H}$ & -7.375932 & 4.298813 & -2.653976 \\
$\mathrm{H}$ & 8.058979 & 3.130739 & -2.622407 \\
$\mathrm{H}$ & -7.383369 & 4.000321 & 2.937307 \\
$\mathrm{H}$ & -5.908977 & -1.241867 & -1.929363 \\
$\mathrm{H}$ & 5.801969 & -2.274525 & 1.775833 \\
$\mathrm{H}$ & -5.986251 & -1.433595 & 1.659891 \\
$\mathrm{H}$ & 5.848815 & -2.010152 & -2.01094 \\
$\mathrm{H}$ & -3.197854 & -3.636767 & -4.230674 \\
$\mathrm{H}$ & 2.745794 & -4.516167 & 3.779425 \\
$\mathrm{H}$ & -3.267402 & -4.111011 & 3.615759 \\
& 2.807794 & -4.002486 & -4.28261 \\
& -5.111273 & -4.802787 & -5.297636 \\
\hline & 4.456688 & -6.075366 & 4.671527
\end{tabular}




\begin{tabular}{|c|c|c|c|}
\hline $\mathrm{H}$ & -5.173721 & -5.36335 & 4.584715 \\
\hline $\mathrm{H}$ & 4.51375 & -5.518327 & -5.260502 \\
\hline $\mathrm{H}$ & -7.841301 & -2.384555 & -2.997381 \\
\hline $\mathrm{H}$ & 7.534047 & -3.813105 & 2.669426 \\
\hline $\mathrm{H}$ & -7.912873 & -2.678628 & 2.612539 \\
\hline $\mathrm{H}$ & 7.575688 & -3.50225 & -2.989753 \\
\hline $\mathrm{H}$ & 7.700003 & 5.154161 & -4.056074 \\
\hline $\mathrm{H}$ & -6.691653 & 6.224594 & -4.102386 \\
\hline $\mathrm{H}$ & 6.878193 & -5.763723 & 4.101192 \\
\hline $\mathrm{H}$ & 6.925501 & -5.297173 & -4.613655 \\
\hline $\mathrm{H}$ & -7.530799 & -4.684336 & 4.061734 \\
\hline $\mathrm{H}$ & -6.748236 & 5.742503 & 4.621501 \\
\hline $\mathrm{H}$ & -7.463088 & -4.205241 & -4.674248 \\
\hline $\mathrm{H}$ & 7.624681 & 4.690944 & 4.63176 \\
\hline $\mathrm{H}$ & -1.866038 & 0.579271 & -2.831781 \\
\hline $\mathrm{H}$ & 2.251378 & 2.938333 & 0.226873 \\
\hline $\mathrm{H}$ & 0.884271 & -1.710475 & 4.216644 \\
\hline $\mathrm{H}$ & 0.055113 & -0.362883 & 4.058269 \\
\hline $\mathrm{H}$ & 0.752253 & -1.163805 & -4.551837 \\
\hline $\mathrm{H}$ & -0.745687 & -3.829216 & -2.711033 \\
\hline $\mathrm{H}$ & -1.91015 & 0.143726 & 2.802448 \\
\hline $\mathrm{H}$ & 1.745507 & -2.815703 & -0.271178 \\
\hline $\mathrm{H}$ & 0.926064 & 1.553869 & 4.483283 \\
\hline $\mathrm{H}$ & 0.160308 & 0.858915 & -4.059018 \\
\hline $\mathrm{H}$ & 1.195539 & 2.0597 & -4.12167 \\
\hline $\mathrm{H}$ & -0.552328 & 4.392334 & 1.894501 \\
\hline $\mathrm{H}$ & -0.32985 & 4.752439 & -1.376572 \\
\hline $\mathrm{H}$ & 0.368029 & 4.245456 & 0.609181 \\
\hline $\mathrm{O}$ & 1.143245 & 0.001193 & 1.455543 \\
\hline 0 & 3.559943 & -1.564561 & 1.245909 \\
\hline $\mathrm{O}$ & 3.594799 & -1.305021 & -1.490072 \\
\hline $\mathrm{O}$ & 3.768384 & 1.174837 & 1.531634 \\
\hline 0 & 2.299171 & 2.315406 & 2.815979 \\
\hline $\mathrm{O}$ & 0.328875 & 4.090445 & 1.614889 \\
\hline $\mathrm{O}$ & -1.765337 & 2.853968 & 2.571555 \\
\hline $\mathrm{O}$ & -1.755697 & 3.179404 & -2.236991 \\
\hline $\mathrm{O}$ & -3.405821 & 1.75573 & 1.477191 \\
\hline $\mathrm{O}$ & -3.394398 & 1.97562 & -1.251349 \\
\hline $\mathrm{O}$ & -3.637662 & -1.026205 & 1.167039 \\
\hline $\mathrm{O}$ & -3.592861 & -0.787435 & -1.491994 \\
\hline 0 & -2.123777 & -2.073508 & -2.63017 \\
\hline $\mathrm{O}$ & -2.198275 & -2.378454 & 2.271388 \\
\hline $\mathrm{O}$ & -1.141645 & -1.328084 & -0.168806 \\
\hline
\end{tabular}




$\begin{array}{lrrr}\mathrm{O} & -1.309027 & 0.12994 & 2.043404 \\ \mathrm{O} & 0.133306 & 1.258639 & 4.012115 \\ \mathrm{O} & 0.018765 & -1.375457 & 3.929276 \\ \mathrm{O} & 0.274555 & 1.856188 & -3.884865 \\ \mathrm{O} & -0.852725 & 1.75153 & 0.128047 \\ \mathrm{O} & 1.634359 & 2.193389 & 0.186729 \\ \mathrm{O} & 2.333206 & 2.678746 & -2.460929 \\ \mathrm{O} & 0.408404 & 4.230277 & -1.030368 \\ \mathrm{O} & 3.798741 & 1.444786 & -1.261426 \\ \mathrm{O} & -0.190152 & -3.746915 & 1.249297 \\ \mathrm{O} & -0.144162 & -3.584217 & -1.989671 \\ \mathrm{O} & 1.296495 & -1.957984 & -0.221996 \\ \mathrm{O} & 1.948957 & -2.502968 & 2.521244 \\ \mathrm{O} & 2.00775 & -2.106165 & -2.882367 \\ \mathrm{O} & 0.004719 & -0.774909 & -4.074532 \\ \mathrm{O} & -1.269282 & 0.502374 & -2.073203 \\ \mathrm{O} & 1.172369 & 0.297927 & -1.478928 \\ \mathrm{Zr} & -2.386354 & 0.385437 & -0.009218 \\ \mathrm{Zr} & 0.239185 & 1.791923 & 1.977772 \\ \mathrm{Zr} & -0.046783 & -1.808489 & 1.662765 \\ \mathrm{Zr} & 0.037514 & -1.346428 & -2.052646 \\ \mathrm{Zr} & 2.593432 & 0.025272 & 0.000568 \\ \mathrm{Zr} & 0.221547 & 2.210134 & -1.593987 \\ \mathrm{Zn} & -1.18478 & -3.550344 & -0.354443 \\ \mathrm{O} & -2.033066 & -5.519912 & 1.9465 \\ \mathrm{H} & -1.599438 & -6.359096 & 1.738835 \\ \mathrm{O} & -3.029475 & -4.401983 & -0.218343 \\ \mathrm{H} & -2.883608 & -4.904082 & 0.650084 \\ \mathrm{H} & -1.286003 & -4.843605 & 1.899954 \\ \mathrm{H} & -3.754584 & -3.774811 & -0.063731\end{array}$


Table S27. Structure for 5-coordinated $\mathrm{Zn}$ with three waters. $E=-5314.30899267$ Hartrees, $G=-5313.606428$ Hartrees

\begin{tabular}{|c|c|c|c|}
\hline Atom & $X$ & $\mathrm{Y}$ & Z \\
\hline$C$ & 5.880001 & 2.565214 & 2.741718 \\
\hline $\mathrm{C}$ & -5.437557 & 3.623571 & -2.337325 \\
\hline$C$ & 5.907984 & 2.892543 & -2.385997 \\
\hline$C$ & -5.431291 & 3.3535 & 2.618833 \\
\hline C & 4.556891 & 2.872964 & 3.091483 \\
\hline C & -4.077151 & 3.841008 & -2.602313 \\
\hline C & 4.589273 & 3.260939 & -2.692068 \\
\hline$C$ & -4.080877 & 3.525597 & 2.957509 \\
\hline $\mathrm{C}$ & 4.319376 & 3.906355 & 4.010295 \\
\hline C & -3.707528 & 4.902019 & -3.442216 \\
\hline C & 4.370964 & 4.369167 & -3.52409 \\
\hline C & -3.738226 & 4.484355 & 3.922067 \\
\hline C & 5.381094 & 4.62574 & 4.55394 \\
\hline C & -4.675744 & 5.74519 & -3.978979 \\
\hline C & 5.443806 & 5.111184 & -4.010494 \\
\hline C & -4.721286 & 5.273707 & 4.512497 \\
\hline C & 6.712858 & 4.327032 & 4.205131 \\
\hline C & -6.041768 & 5.556751 & -3.691685 \\
\hline C & 6.770655 & 4.765672 & -3.686596 \\
\hline$C$ & -6.077632 & 5.124943 & 4.161447 \\
\hline$C$ & 6.939595 & 3.277835 & 3.293721 \\
\hline C & -6.403247 & 4.466791 & -2.877826 \\
\hline C & 6.979275 & 3.630545 & -2.879765 \\
\hline C & -6.41327 & 4.137167 & 3.216273 \\
\hline$C$ & -5.74163 & -2.014219 & -2.662751 \\
\hline C & 5.545695 & -2.936803 & 2.418527 \\
\hline C & -5.809585 & -2.285059 & 2.301966 \\
\hline C & 5.590054 & -2.60632 & -2.73305 \\
\hline$C$ & -4.421228 & -2.351949 & -2.994864 \\
\hline C & 4.19101 & -3.148046 & 2.717174 \\
\hline C & -4.487345 & -2.669258 & 2.570504 \\
\hline C & 4.240933 & -2.755987 & -3.0901 \\
\hline C & -4.192645 & -3.34527 & -3.95792 \\
\hline C & 3.840192 & -4.220068 & 3.552105 \\
\hline C & -4.253289 & -3.766447 & 3.412525 \\
\hline C & 3.890286 & -3.751753 & -4.014519 \\
\hline $\mathrm{C}$ & -5.261205 & -4.008858 & -4.553081 \\
\hline C & 4.81728 & -5.081513 & 4.043542 \\
\hline C & -5.319555 & -4.482779 & 3.947649 \\
\hline
\end{tabular}




\begin{tabular}{|c|c|c|c|}
\hline C & 4.863547 & -4.590362 & -4.552954 \\
\hline C & -6.590282 & -3.695977 & -4.20742 \\
\hline C & 6.177238 & -4.896078 & 3.72509 \\
\hline C & -6.65086 & -4.126967 & 3.656217 \\
\hline C & 6.218995 & -4.454126 & -4.193996 \\
\hline C & -6.808497 & -2.673316 & -3.265049 \\
\hline C & 6.521717 & -3.793381 & 2.919166 \\
\hline C & -6.873668 & -3.002287 & 2.839964 \\
\hline C & 6.562027 & -3.438746 & -3.280434 \\
\hline C & 3.454885 & 2.490174 & -2.10372 \\
\hline C & 3.427974 & 2.15381 & 2.438344 \\
\hline C & 3.157895 & -2.25185 & 2.117052 \\
\hline C & -3.344991 & -1.938406 & 1.958051 \\
\hline C & -3.288845 & -1.657488 & -2.316595 \\
\hline C & -3.035177 & 2.974242 & -1.987018 \\
\hline C & 3.199523 & -1.913751 & -2.435738 \\
\hline C & -3.038751 & 2.69379 & 2.287706 \\
\hline $\mathrm{H}$ & 6.057407 & 1.767923 & 2.023795 \\
\hline $\mathrm{H}$ & -5.717192 & 2.792068 & -1.694374 \\
\hline $\mathrm{H}$ & 6.073125 & 2.028765 & -1.746599 \\
\hline $\mathrm{H}$ & -5.690192 & 2.600922 & 1.87789 \\
\hline $\mathrm{H}$ & 3.291884 & 4.151243 & 4.269927 \\
\hline $\mathrm{H}$ & -2.653486 & 5.05467 & -3.661838 \\
\hline $\mathrm{H}$ & 3.347618 & 4.655265 & -3.757995 \\
\hline $\mathrm{H}$ & -2.692852 & 4.59931 & 4.200656 \\
\hline $\mathrm{H}$ & 5.175872 & 5.432316 & 5.256279 \\
\hline $\mathrm{H}$ & -4.370216 & 6.568872 & -4.622708 \\
\hline $\mathrm{H}$ & 5.251063 & 5.978125 & -4.640764 \\
\hline $\mathrm{H}$ & -4.435449 & 6.017605 & 5.254716 \\
\hline $\mathrm{H}$ & 7.95982 & 3.024759 & 3.008493 \\
\hline $\mathrm{H}$ & -7.454071 & 4.287749 & -2.654327 \\
\hline $\mathrm{H}$ & 7.995474 & 3.331948 & -2.625518 \\
\hline $\mathrm{H}$ & -7.456145 & 3.992528 & 2.937256 \\
\hline $\mathrm{H}$ & -5.910128 & -1.236544 & -1.921728 \\
\hline $\mathrm{H}$ & 5.813391 & -2.101386 & 1.776027 \\
\hline $\mathrm{H}$ & -5.983415 & -1.426327 & 1.657576 \\
\hline $\mathrm{H}$ & 5.856191 & -1.837905 & -2.011138 \\
\hline $\mathrm{H}$ & -3.168 & -3.586505 & -4.232992 \\
\hline $\mathrm{H}$ & 2.788609 & -4.380627 & 3.782405 \\
\hline $\mathrm{H}$ & -3.227483 & -4.060049 & 3.619951 \\
\hline $\mathrm{H}$ & 2.84193 & -3.872956 & -4.280048 \\
\hline $\mathrm{H}$ & -5.065485 & -4.781743 & -5.295115 \\
\hline $\mathrm{H}$ & 4.522408 & -5.918547 & 4.675149 \\
\hline
\end{tabular}




\begin{tabular}{|c|c|c|c|}
\hline $\mathrm{H}$ & -5.119035 & -5.338544 & 4.590664 \\
\hline $\mathrm{H}$ & 4.568748 & -5.366122 & -5.258286 \\
\hline $\mathrm{H}$ & -7.827702 & -2.402075 & -2.993059 \\
\hline $\mathrm{H}$ & 7.567285 & -3.615227 & 2.671119 \\
\hline $\mathrm{H}$ & -7.89382 & -2.695113 & 2.613856 \\
\hline $\mathrm{H}$ & 7.603353 & -3.307042 & -2.989352 \\
\hline $\mathrm{H}$ & 7.608405 & 5.349456 & -4.060233 \\
\hline $\mathrm{H}$ & -6.796625 & 6.222005 & -4.103955 \\
\hline $\mathrm{H}$ & 6.93839 & -5.573904 & 4.103767 \\
\hline $\mathrm{H}$ & 6.977506 & -5.111569 & -4.612042 \\
\hline $\mathrm{H}$ & -7.483615 & -4.693178 & 4.066721 \\
\hline $\mathrm{H}$ & -6.844606 & 5.744334 & 4.620262 \\
\hline $\mathrm{H}$ & -7.42486 & -4.21737 & -4.670138 \\
\hline $\mathrm{H}$ & 7.541392 & 4.890306 & 4.627752 \\
\hline $\mathrm{H}$ & -1.889753 & 0.639028 & -2.832108 \\
\hline $\mathrm{H}$ & 2.192222 & 3.046406 & 0.227225 \\
\hline $\mathrm{H}$ & 0.877562 & -1.627137 & 4.153863 \\
\hline $\mathrm{H}$ & 0.018567 & -0.301264 & 4.028845 \\
\hline $\mathrm{H}$ & 0.746959 & -1.083688 & -4.513543 \\
\hline $\mathrm{H}$ & -0.896228 & -3.70508 & -2.645878 \\
\hline $\mathrm{H}$ & -1.936756 & 0.220888 & 2.806436 \\
\hline $\mathrm{H}$ & 1.904598 & -2.650651 & -0.252338 \\
\hline $\mathrm{H}$ & 0.885455 & 1.629077 & 4.48597 \\
\hline $\mathrm{H}$ & 0.129912 & 0.922331 & -4.044618 \\
\hline $\mathrm{H}$ & 1.142647 & 2.144693 & -4.128438 \\
\hline $\mathrm{H}$ & -0.628803 & 4.464592 & 1.888869 \\
\hline $\mathrm{H}$ & -0.410275 & 4.824024 & -1.377842 \\
\hline $\mathrm{H}$ & 0.293285 & 4.325559 & 0.604349 \\
\hline 0 & 1.113528 & 0.089241 & 1.452426 \\
\hline 0 & 3.56057 & -1.443921 & 1.221665 \\
\hline $\mathrm{O}$ & 3.593683 & -1.164538 & -1.487538 \\
\hline 0 & 3.732263 & 1.315321 & 1.533792 \\
\hline 0 & 2.248006 & 2.437232 & 2.815332 \\
\hline 0 & 0.256967 & 4.174687 & 1.611167 \\
\hline $\mathrm{O}$ & -1.822972 & 2.917056 & 2.577142 \\
\hline 0 & -1.818508 & 3.24363 & -2.238294 \\
\hline 0 & -3.449593 & 1.807457 & 1.47363 \\
\hline $\mathrm{O}$ & -3.442265 & 2.02047 & -1.252241 \\
\hline 0 & -3.63507 & -0.961577 & 1.200228 \\
\hline $\mathrm{O}$ & -3.596184 & -0.765238 & -1.466165 \\
\hline $\mathrm{O}$ & -2.114378 & -1.993255 & -2.647403 \\
\hline $\mathrm{O}$ & -2.18268 & -2.356812 & 2.231703 \\
\hline $\mathrm{O}$ & -1.12898 & -1.222382 & -0.160216 \\
\hline
\end{tabular}




\begin{tabular}{lrrr}
$\mathrm{O}$ & -1.335799 & 0.200876 & 2.047668 \\
$\mathrm{O}$ & 0.0959 & 1.332457 & 4.010564 \\
$\mathrm{O}$ & -0.004567 & -1.311517 & 3.89369 \\
$\mathrm{O}$ & 0.227539 & 1.926637 & -3.883043 \\
$\mathrm{O}$ & -0.89678 & 1.842448 & 0.131034 \\
$\mathrm{O}$ & 1.585827 & 2.292818 & 0.186511 \\
$\mathrm{O}$ & 2.276343 & 2.795243 & -2.465614 \\
$\mathrm{O}$ & 0.337601 & 4.313593 & -1.034734 \\
$\mathrm{O}$ & 3.759089 & 1.589527 & -1.260706 \\
$\mathrm{O}$ & -0.034155 & -3.726091 & 1.364287 \\
$\mathrm{O}$ & -0.100299 & -3.543391 & -2.111146 \\
$\mathrm{O}$ & 1.309471 & -1.88796 & -0.224462 \\
$\mathrm{O}$ & 1.972168 & -2.357183 & 2.53959 \\
$\mathrm{O}$ & 2.014773 & -1.997849 & -2.869313 \\
$\mathrm{O}$ & -0.009159 & -0.696193 & -4.048544 \\
$\mathrm{O}$ & -1.298766 & 0.571533 & -2.068163 \\
$\mathrm{O}$ & 1.138622 & 0.385101 & -1.472599 \\
$\mathrm{Zr}$ & -2.40416 & 0.4554 & -0.006865 \\
$\mathrm{Zr}$ & 0.194331 & 1.874813 & 1.979839 \\
$\mathrm{Zr}$ & -0.061128 & -1.737654 & 1.616772 \\
$\mathrm{Zr}$ & 0.010414 & -1.290247 & -2.028154 \\
$\mathrm{Zr}$ & 2.566927 & 0.153972 & -0.001682 \\
$\mathrm{Zr}$ & 0.172705 & 2.289775 & -1.598327 \\
$\mathrm{Zn}$ & -0.474424 & -4.218549 & -0.368876 \\
$\mathrm{O}$ & -1.481323 & -5.711278 & 2.276238 \\
$\mathrm{H}$ & -1.017137 & -6.207084 & 2.963245 \\
$\mathrm{O}$ & -2.62915 & -3.752691 & -0.299363 \\
$\mathrm{H}$ & -2.820366 & -3.941836 & 0.637109 \\
$\mathrm{H}$ & -0.926895 & -4.87662 & 2.137911 \\
$\mathrm{H}$ & -2.456475 & -2.781601 & -0.295181 \\
$\mathrm{O}$ & -0.93367 & -6.25889 & -0.256843 \\
$\mathrm{H}$ & -1.16728 & -6.279314 & 0.723451 \\
$\mathrm{H}$ & -1.769378 & -6.42059 & -0.720258 \\
& & & \\
\hline
\end{tabular}


Table S28. Structure for 6-coordinated $\mathrm{Zn}$ with four waters. $E=-5390.73839474$ Hartrees, $G=-5390.010648$ Hartrees

\begin{tabular}{|c|c|c|c|}
\hline Atom & $\mathrm{X}$ & $\mathrm{Y}$ & Z \\
\hline C & 6.037402 & 2.592619 & 2.516059 \\
\hline $\mathrm{C}$ & -5.281508 & 3.685243 & -2.552676 \\
\hline $\mathrm{C}$ & 6.025088 & 2.496092 & -2.621397 \\
\hline $\mathrm{C}$ & -5.234489 & 3.823431 & 2.408119 \\
\hline C & 4.731211 & 2.981216 & 2.848388 \\
\hline $\mathrm{C}$ & -3.916562 & 3.824723 & -2.844656 \\
\hline C & 4.719063 & 2.890569 & -2.947506 \\
\hline C & -3.875477 & 3.96764 & 2.72187 \\
\hline C & 4.544273 & 4.095215 & 3.680679 \\
\hline C & -3.513856 & 4.797459 & -3.771932 \\
\hline $\mathrm{C}$ & 4.53596 & 3.934705 & -3.866785 \\
\hline C & -3.484627 & 4.988424 & 3.601636 \\
\hline C & 5.639238 & 4.81375 & 4.155185 \\
\hline C & -4.453478 & 5.632112 & -4.369426 \\
\hline C & 5.63219 & 4.590576 & -4.420198 \\
\hline C & -4.429478 & 5.862459 & 4.132029 \\
\hline C & 6.954442 & 4.433965 & 3.822828 \\
\hline C & -5.822839 & 5.523043 & -4.057952 \\
\hline C & 6.947628 & 4.219676 & -4.07831 \\
\hline C & -5.794249 & 5.740019 & 3.804163 \\
\hline C & 7.130033 & 3.305022 & 2.999686 \\
\hline C & -6.218685 & 4.5191 & -3.154372 \\
\hline C & 7.119545 & 3.147206 & -3.182068 \\
\hline C & -6.178466 & 4.692292 & 2.946073 \\
\hline C & -5.81174 & -1.943024 & -2.410327 \\
\hline C & 5.482505 & -2.898463 & 2.651379 \\
\hline C & -5.838663 & -1.800447 & 2.563474 \\
\hline C & 5.486541 & -2.99438 & -2.512487 \\
\hline C & -4.509201 & -2.360268 & -2.722402 \\
\hline C & 4.122133 & -3.031511 & 2.975819 \\
\hline C & -4.53254 & -2.213786 & 2.848906 \\
\hline C & 4.127835 & -3.120229 & -2.845226 \\
\hline C & -4.330203 & -3.438127 & -3.601231 \\
\hline C & 3.739201 & -4.015267 & 3.898072 \\
\hline C & -4.330683 & -3.25006 & 3.777829 \\
\hline C & 3.72931 & -4.172995 & -3.681509 \\
\hline C & -5.430238 & -4.104545 & -4.132351 \\
\hline C & 4.686679 & -4.871855 & 4.452182 \\
\hline C & -5.419176 & -3.875094 & 4.379136 \\
\hline
\end{tabular}




\begin{tabular}{|c|c|c|c|}
\hline C & 4.6631 & -5.091276 & -4.156112 \\
\hline$C$ & -6.742244 & -3.711202 & -3.804086 \\
\hline$C$ & 6.049435 & -4.768415 & 4.109593 \\
\hline C & -6.738261 & -3.491447 & 4.068326 \\
\hline C & 6.02643 & -4.98101 & -3.818804 \\
\hline$C$ & -6.910047 & -2.606658 & -2.947748 \\
\hline C & 6.428888 & -3.750539 & 3.212854 \\
\hline$C$ & -6.924827 & -2.429909 & 3.163207 \\
\hline$C$ & 6.418807 & -3.90871 & -2.994502 \\
\hline C & 3.56179 & 2.218369 & -2.290586 \\
\hline C & 3.569806 & 2.25843 & 2.263825 \\
\hline C & 3.124087 & -2.143051 & 2.301612 \\
\hline$C$ & -3.372566 & -1.594535 & 2.173207 \\
\hline C & -3.341849 & -1.658913 & -2.109042 \\
\hline C & -2.902766 & 2.971101 & -2.16903 \\
\hline C & 3.129188 & -2.185638 & -2.247468 \\
\hline C & -2.871475 & 3.044162 & 2.118402 \\
\hline $\mathrm{H}$ & 6.175719 & 1.732599 & 1.865079 \\
\hline $\mathrm{H}$ & -5.587143 & 2.922128 & -1.840456 \\
\hline $\mathrm{H}$ & 6.162703 & 1.682428 & -1.9134 \\
\hline $\mathrm{H}$ & -5.530927 & 3.023507 & 1.73368 \\
\hline $\mathrm{H}$ & 3.529901 & 4.40148 & 3.92635 \\
\hline $\mathrm{H}$ & -2.457085 & 4.888782 & -4.01136 \\
\hline $\mathrm{H}$ & 3.522396 & 4.241728 & -4.116012 \\
\hline $\mathrm{H}$ & -2.432816 & 5.083441 & 3.862736 \\
\hline $\mathrm{H}$ & 5.473418 & 5.683014 & 4.789846 \\
\hline $\mathrm{H}$ & -4.122237 & 6.38723 & -5.080949 \\
\hline $\mathrm{H}$ & 5.467403 & 5.410018 & -5.118296 \\
\hline $\mathrm{H}$ & -4.106837 & 6.652661 & 4.808429 \\
\hline $\mathrm{H}$ & 8.136514 & 2.988482 & 2.729302 \\
\hline $\mathrm{H}$ & -7.273316 & 4.401601 & -2.909111 \\
\hline $\mathrm{H}$ & 8.125801 & 2.830289 & -2.910908 \\
\hline $\mathrm{H}$ & -7.229034 & 4.567049 & 2.687422 \\
\hline $\mathrm{H}$ & -5.941825 & -1.100693 & -1.735134 \\
\hline $\mathrm{H}$ & 5.776123 & -2.128099 & 1.942463 \\
\hline $\mathrm{H}$ & -5.987831 & -0.990775 & 1.852257 \\
\hline $\mathrm{H}$ & 5.788497 & -2.179257 & -1.85918 \\
\hline $\mathrm{H}$ & -3.322173 & -3.726434 & -3.886929 \\
\hline $\mathrm{H}$ & 2.684803 & -4.115823 & 4.149094 \\
\hline $\mathrm{H}$ & -3.318221 & -3.566321 & 4.003405 \\
\hline $\mathrm{H}$ & 2.679229 & -4.265361 & -3.953076 \\
\hline $\mathrm{H}$ & -5.270828 & -4.940653 & -4.811685 \\
\hline $\mathrm{H}$ & 4.364616 & -5.640546 & 5.153345 \\
\hline
\end{tabular}




\begin{tabular}{|c|c|c|c|}
\hline $\mathrm{H}$ & -5.244753 & -4.683252 & 5.087807 \\
\hline $\mathrm{H}$ & 4.332222 & -5.905395 & -4.799482 \\
\hline $\mathrm{H}$ & -7.914775 & -2.272867 & -2.692024 \\
\hline $\mathrm{H}$ & 7.478379 & -3.634777 & 2.944788 \\
\hline $\mathrm{H}$ & -7.934204 & -2.100828 & 2.919904 \\
\hline $\mathrm{H}$ & 7.467457 & -3.795131 & -2.723408 \\
\hline $\mathrm{H}$ & 7.803922 & 4.736722 & -4.504548 \\
\hline $\mathrm{H}$ & -6.55509 & 6.181964 & -4.51834 \\
\hline $\mathrm{H}$ & 6.787254 & -5.44264 & 4.537757 \\
\hline $\mathrm{H}$ & 6.753706 & -5.699985 & -4.187753 \\
\hline $\mathrm{H}$ & -7.588879 & -3.988771 & 4.528712 \\
\hline $\mathrm{H}$ & -6.531364 & 6.425171 & 4.21601 \\
\hline $\mathrm{H}$ & -7.601742 & -4.234586 & -4.216119 \\
\hline $\mathrm{H}$ & 7.808952 & 4.996388 & 4.191389 \\
\hline $\mathrm{H}$ & -1.875316 & 0.542478 & -2.825991 \\
\hline $\mathrm{H}$ & 2.347215 & 3.014838 & -0.006729 \\
\hline $\mathrm{H}$ & 0.877827 & -1.265168 & 4.32685 \\
\hline $\mathrm{H}$ & 0.086063 & 0.087368 & 4.082069 \\
\hline $\mathrm{H}$ & 0.667854 & -1.43116 & -4.387553 \\
\hline $\mathrm{H}$ & 0.754781 & -3.925755 & -1.987265 \\
\hline $\mathrm{H}$ & -1.8679 & 0.578025 & 2.835072 \\
\hline $\mathrm{H}$ & 1.82159 & -2.659317 & 0.047474 \\
\hline $\mathrm{H}$ & 1.032236 & 2.014412 & 4.365592 \\
\hline $\mathrm{H}$ & 0.160675 & 0.646623 & -4.072878 \\
\hline $\mathrm{H}$ & 1.21875 & 1.818324 & -4.253788 \\
\hline $\mathrm{H}$ & -0.398591 & 4.683939 & 1.55546 \\
\hline $\mathrm{H}$ & -0.204631 & 4.762714 & -1.731803 \\
\hline $\mathrm{H}$ & 0.503194 & 4.401558 & 0.280334 \\
\hline 0 & 1.164622 & 0.226495 & 1.479082 \\
\hline 0 & 3.553535 & -1.435652 & 1.334217 \\
\hline $\mathrm{O}$ & 3.562095 & -1.349787 & -1.405319 \\
\hline 0 & 3.830691 & 1.338286 & 1.427263 \\
\hline 0 & 2.406278 & 2.620625 & 2.62345 \\
\hline 0 & 0.471664 & 4.335847 & 1.296038 \\
\hline $\mathrm{O}$ & -1.645212 & 3.246607 & 2.375621 \\
\hline 0 & -1.679448 & 3.171646 & -2.452357 \\
\hline 0 & -3.325703 & 2.108342 & 1.386434 \\
\hline $\mathrm{O}$ & -3.337963 & 2.102302 & -1.350693 \\
\hline $\mathrm{O}$ & -3.627958 & -0.68624 & 1.315992 \\
\hline $\mathrm{O}$ & -3.607375 & -0.673 & -1.352334 \\
\hline $\mathrm{O}$ & -2.187917 & -2.099552 & -2.383419 \\
\hline $\mathrm{O}$ & -2.221087 & -2.01897 & 2.477292 \\
\hline $\mathrm{O}$ & -1.136288 & -1.123656 & -0.014502 \\
\hline
\end{tabular}




\begin{tabular}{lrrr}
$\mathrm{O}$ & -1.277503 & 0.477923 & 2.074266 \\
$\mathrm{O}$ & 0.223585 & 1.713361 & 3.926399 \\
$\mathrm{O}$ & 0.015802 & -0.927657 & 4.030701 \\
$\mathrm{O}$ & 0.295533 & 1.655881 & -3.995207 \\
$\mathrm{O}$ & -0.790202 & 1.92838 & 0.021943 \\
$\mathrm{O}$ & 1.706565 & 2.289561 & 0.020813 \\
$\mathrm{O}$ & 2.392744 & 2.541451 & -2.667941 \\
$\mathrm{O}$ & 0.526435 & 4.252542 & -1.353842 \\
$\mathrm{O}$ & 3.836208 & 1.380599 & -1.375587 \\
$\mathrm{O}$ & -0.135095 & -3.535786 & 1.683799 \\
$\mathrm{O}$ & -0.157397 & -3.649948 & -1.802484 \\
$\mathrm{O}$ & 1.274772 & -1.865153 & -0.028275 \\
$\mathrm{O}$ & 1.940225 & -2.158056 & 2.739593 \\
$\mathrm{O}$ & 1.923653 & -2.311877 & -2.630563 \\
$\mathrm{O}$ & -0.044208 & -0.958184 & -3.933736 \\
$\mathrm{O}$ & -1.274476 & 0.508907 & -2.067641 \\
$\mathrm{O}$ & 1.166258 & 0.269922 & -1.482285 \\
$\mathrm{Zr}$ & -2.358586 & 0.595796 & 0.015028 \\
$\mathrm{Zr}$ & 0.317028 & 2.078801 & 1.857587 \\
$\mathrm{Zr}$ & -0.085956 & -1.543755 & 1.802127 \\
$\mathrm{Zr}$ & -0.051988 & -1.384416 & -1.87104 \\
$\mathrm{Zr}$ & 2.591091 & 0.112082 & 0.011439 \\
$\mathrm{Zr}$ & 0.272553 & 2.198465 & -1.744872 \\
$\mathrm{Zn}$ & -0.735348 & -4.068798 & 0.016866 \\
$\mathrm{O}$ & -1.029849 & -6.178265 & -0.147981 \\
$\mathrm{H}$ & -1.505252 & -6.138621 & -1.03792 \\
$\mathrm{O}$ & -2.823822 & -3.752857 & -0.012456 \\
$\mathrm{H}$ & -2.957683 & -4.125851 & 0.907155 \\
$\mathrm{H}$ & -0.220571 & -6.688182 & -0.296448 \\
$\mathrm{H}$ & -2.842382 & -2.779264 & 0.08354 \\
$\mathrm{O}$ & -2.16714 & -5.464286 & -2.411572 \\
$\mathrm{H}$ & -2.968497 & -5.018216 & -2.088696 \\
$\mathrm{H}$ & -1.530054 & -4.717494 & -2.502321 \\
\hline & -2.341553 & -5.025803 & 2.308365 \\
$\mathrm{H}$ & -1.990396 & -5.847513 & 1.931661 \\
$\mathrm{H}$ & & -4.448306 & 2.387205
\end{tabular}


Table S29. Structure for $2\left[\mathrm{H}_{2} \mathrm{O}, \mathrm{OH}\right]$.

$E=-5310.94822884$ Hartrees, $G=-5310.340308$ Hartrees

\begin{tabular}{|c|c|c|c|}
\hline Atom & $x$ & $Y$ & $Z$ \\
\hline $\mathrm{C}$ & -5.663528 & -2.792785 & 2.553482 \\
\hline $\mathrm{C}$ & 5.713616 & -2.770343 & -2.502503 \\
\hline C & -5.655258 & -2.771839 & -2.584508 \\
\hline $\mathrm{C}$ & 5.675377 & -2.838914 & 2.460305 \\
\hline C & -4.324219 & -3.037603 & 2.891283 \\
\hline $\mathrm{C}$ & 4.370967 & -3.056546 & -2.791701 \\
\hline$C$ & -4.314696 & -3.032559 & -2.905075 \\
\hline C & 4.338079 & -3.120434 & 2.776584 \\
\hline C & -4.022837 & -4.113553 & 3.739734 \\
\hline $\mathrm{C}$ & 4.073469 & -4.079807 & -3.703945 \\
\hline C & -4.022328 & -4.065206 & -3.808833 \\
\hline C & 4.055498 & -4.162725 & 3.671601 \\
\hline C & -5.037212 & -4.935205 & 4.225774 \\
\hline C & 5.095909 & -4.820188 & -4.289509 \\
\hline C & -5.043304 & -4.840234 & -4.352051 \\
\hline C & 5.086001 & -4.925235 & 4.214494 \\
\hline $\mathrm{C}$ & -6.384536 & -4.700063 & 3.88827 \\
\hline C & 6.445983 & -4.563899 & -3.980163 \\
\hline C & -6.390724 & -4.603905 & -4.015292 \\
\hline C & 6.430851 & -4.665662 & 3.884269 \\
\hline C & -6.676246 & -3.608154 & 3.048306 \\
\hline C & 6.73357 & -3.510762 & -3.091778 \\
\hline C & -6.674943 & -3.542063 & -3.135124 \\
\hline C & 6.704412 & -3.596292 & 3.010587 \\
\hline C & 5.652014 & 2.884461 & -2.443384 \\
\hline C & -5.686147 & 2.728678 & 2.605735 \\
\hline C & 5.687775 & 2.820867 & 2.52847 \\
\hline C & -5.69393 & 2.747128 & -2.556323 \\
\hline C & 4.313492 & 3.157861 & -2.761685 \\
\hline C & -4.348929 & 3.006069 & 2.927968 \\
\hline C & 4.342549 & 3.099703 & 2.812851 \\
\hline C & -4.356326 & 3.007385 & -2.892765 \\
\hline C & 4.023701 & 4.197514 & -3.657043 \\
\hline C & -4.070863 & 4.039202 & 3.836138 \\
\hline C & 4.035362 & 4.119618 & 3.725428 \\
\hline C & -4.068392 & 4.084218 & -3.745102 \\
\hline C & 5.048638 & 4.967752 & -4.197986 \\
\hline C & -5.103234 & 4.80039 & 4.377532 \\
\hline$C$ & 5.051326 & 4.865312 & 4.315508 \\
\hline
\end{tabular}




\begin{tabular}{|c|c|c|c|}
\hline$C$ & -5.092456 & 4.892976 & -4.232569 \\
\hline$C$ & 6.394212 & 4.718557 & -3.864569 \\
\hline$C$ & -6.447355 & 4.549611 & 4.037329 \\
\hline$C$ & 6.403762 & 4.616838 & 4.010422 \\
\hline$C$ & -6.437247 & 4.645388 & -3.89362 \\
\hline$C$ & 6.675603 & 3.650385 & -2.99202 \\
\hline$C$ & -6.717173 & 3.484329 & 3.156143 \\
\hline$C$ & 6.701485 & 3.566992 & 3.121601 \\
\hline$C$ & -6.716307 & 3.550203 & -3.053406 \\
\hline$C$ & -3.232493 & -2.231794 & -2.258296 \\
\hline$C$ & -3.242624 & -2.203706 & 2.296928 \\
\hline$C$ & -3.257968 & 2.220223 & 2.27681 \\
\hline$C$ & 3.252139 & 2.339018 & 2.144558 \\
\hline C & 3.226204 & 2.346904 & -2.14061 \\
\hline$C$ & 3.27159 & -2.301952 & -2.126684 \\
\hline$C$ & -3.263935 & 2.190949 & -2.289169 \\
\hline$C$ & 3.245525 & -2.313768 & 2.157434 \\
\hline $\mathrm{H}$ & -5.890619 & -1.96223 & 1.889086 \\
\hline $\mathrm{H}$ & 5.935982 & -1.969079 & -1.801183 \\
\hline $\mathrm{H}$ & -5.877752 & -1.967344 & -1.887419 \\
\hline $\mathrm{H}$ & 5.886254 & -2.022496 & 1.773524 \\
\hline $\mathrm{H}$ & -2.98168 & -4.306804 & 3.990337 \\
\hline $\mathrm{H}$ & 3.032033 & -4.307899 & -3.918863 \\
\hline $\mathrm{H}$ & -2.982117 & -4.273893 & -4.050921 \\
\hline $\mathrm{H}$ & 3.020409 & -4.3624 & 3.940856 \\
\hline $\mathrm{H}$ & -4.781973 & -5.772613 & 4.873459 \\
\hline $\mathrm{H}$ & 4.846313 & -5.621301 & -4.983965 \\
\hline $\mathrm{H}$ & -4.792831 & -5.649191 & -5.036726 \\
\hline $\mathrm{H}$ & 4.847173 & -5.733731 & 4.903953 \\
\hline $\mathrm{H}$ & -7.710111 & -3.401561 & 2.775068 \\
\hline $\mathrm{H}$ & 7.769982 & -3.279564 & -2.849685 \\
\hline $\mathrm{H}$ & -7.709203 & -3.326988 & -2.869799 \\
\hline $\mathrm{H}$ & 7.736452 & -3.364946 & 2.750735 \\
\hline $\mathrm{H}$ & 5.86901 & 2.070524 & -1.755576 \\
\hline $\mathrm{H}$ & -5.897967 & 1.921696 & 1.908135 \\
\hline $\mathrm{H}$ & 5.918567 & 2.023052 & 1.825856 \\
\hline $\mathrm{H}$ & -5.911216 & 1.915249 & -1.890439 \\
\hline $\mathrm{H}$ & 2.982838 & 4.39335 & -3.907082 \\
\hline $\mathrm{H}$ & -3.033306 & 4.254423 & 4.08398 \\
\hline $\mathrm{H}$ & 2.990081 & 4.32436 & 3.945218 \\
\hline $\mathrm{H}$ & -3.034119 & 4.279913 & -4.021775 \\
\hline $\mathrm{H}$ & 4.804333 & 5.777136 & -4.884553 \\
\hline $\mathrm{H}$ & -4.863616 & 5.609858 & 5.065456 \\
\hline
\end{tabular}




\begin{tabular}{|c|c|c|c|}
\hline $\mathrm{H}$ & 4.794065 & 5.661701 & 5.012486 \\
\hline $\mathrm{H}$ & -4.846864 & 5.728305 & -4.886579 \\
\hline $\mathrm{H}$ & 7.709364 & 3.429272 & -2.729262 \\
\hline $\mathrm{H}$ & -7.74817 & 3.255852 & 2.889136 \\
\hline $\mathrm{H}$ & 7.740268 & 3.342853 & 2.882502 \\
\hline $\mathrm{H}$ & -7.74763 & 3.331369 & -2.780413 \\
\hline $\mathrm{H}$ & -7.187501 & -5.213785 & -4.433876 \\
\hline $\mathrm{H}$ & 7.243438 & -5.150249 & -4.430184 \\
\hline $\mathrm{H}$ & -7.252156 & 5.14926 & 4.455457 \\
\hline $\mathrm{H}$ & -7.235349 & 5.278565 & -4.273088 \\
\hline $\mathrm{H}$ & 7.196656 & 5.207166 & 4.463774 \\
\hline $\mathrm{H}$ & 7.234905 & -5.2636 & 4.30643 \\
\hline $\mathrm{H}$ & 7.194842 & 5.323868 & -4.283287 \\
\hline $\mathrm{H}$ & -7.176032 & -5.342648 & 4.266227 \\
\hline $\mathrm{H}$ & 1.947842 & -0.026557 & -2.868329 \\
\hline $\mathrm{H}$ & -2.150699 & -2.744885 & 0.059579 \\
\hline $\mathrm{H}$ & -0.964381 & 1.608793 & 4.302754 \\
\hline $\mathrm{H}$ & 2.014029 & 0.036341 & 2.815877 \\
\hline $\mathrm{H}$ & -2.155421 & 2.744501 & -0.008525 \\
\hline $\mathrm{H}$ & 0.849767 & -4.232846 & 2.278892 \\
\hline 0 & -1.060267 & 0.055447 & 1.487367 \\
\hline 0 & -3.598639 & 1.441866 & 1.326983 \\
\hline 0 & -3.598925 & 1.335691 & -1.41585 \\
\hline 0 & -3.584423 & -1.369828 & 1.400536 \\
\hline 0 & -2.061133 & -2.385346 & 2.720218 \\
\hline 0 & -0.012084 & -4.035696 & 1.869427 \\
\hline $\mathrm{O}$ & 2.048566 & -2.629174 & 2.444637 \\
\hline $\mathrm{O}$ & 2.092543 & -2.557492 & -2.510807 \\
\hline 0 & 3.590343 & -1.347958 & 1.411644 \\
\hline 0 & 3.599038 & -1.435681 & -1.260083 \\
\hline $\mathrm{O}$ & 3.594323 & 1.456808 & 1.289406 \\
\hline 0 & 3.586457 & 1.365598 & -1.406778 \\
\hline 0 & 2.030951 & 2.668593 & -2.389169 \\
\hline 0 & 2.068977 & 2.592142 & 2.504701 \\
\hline $\mathrm{O}$ & 1.023604 & 1.557246 & -0.036375 \\
\hline 0 & 1.382273 & 0.048999 & 2.081865 \\
\hline 0 & 0.041595 & -1.277566 & 4.003545 \\
\hline $\mathrm{O}$ & -0.062816 & 1.366027 & 4.032444 \\
\hline $\mathrm{O}$ & 0.107402 & -2.093201 & -4.102759 \\
\hline $\mathrm{O}$ & 1.0353 & -1.553274 & 0.047214 \\
\hline $\mathrm{O}$ & -1.356464 & -2.190467 & 0.091352 \\
\hline $\mathrm{O}$ & -2.052186 & -2.377465 & -2.693548 \\
\hline $\mathrm{O}$ & -0.025397 & -3.934737 & -1.686399 \\
\hline
\end{tabular}




$\begin{array}{llll}\mathrm{O} & -3.575466 & -1.470257 & -1.299126 \\ \mathrm{O} & -0.024408 & 3.967026 & 1.623265 \\ \mathrm{O} & -0.128471 & 4.06536 & -1.930672 \\ \mathrm{O} & -1.386835 & 2.157396 & -0.070159 \\ \mathrm{O} & -2.074728 & 2.400201 & 2.68866 \\ \mathrm{O} & -2.0839 & 2.429434 & -2.688587 \\ \mathrm{O} & 0.083552 & 1.44753 & -3.992568 \\ \mathrm{O} & 1.369055 & -0.046001 & -2.091688 \\ \mathrm{O} & -1.074652 & -0.047923 & -1.499589 \\ \mathrm{Zr} & 2.451384 & 0.03287 & 0.001184 \\ \mathrm{Zr} & -0.028508 & -1.688681 & 1.952088 \\ \mathrm{Zr} & 0.028817 & 1.944414 & 1.798188 \\ \mathrm{Zr} & -0.020871 & 1.719272 & -1.999912 \\ \mathrm{Zr} & -2.461882 & 0.006945 & 0.005221 \\ \mathrm{Zr} & 0.020552 & -1.882865 & -1.769105 \\ \mathrm{Zn} & 0.037125 & 4.224188 & -0.114623 \\ \mathrm{Zn} & 0.076431 & -4.200228 & 0.049316 \\ \mathrm{H} & -1.038515 & 4.261787 & -2.210832 \\ \mathrm{H} & 0.835508 & -2.72947 & -4.217793 \\ \mathrm{H} & -0.717597 & -1.669347 & 4.459768 \\ \mathrm{H} & -0.007416 & 0.355401 & 4.125901 \\ \mathrm{H} & -0.734778 & 1.586483 & -4.487483 \\ \mathrm{H} & -0.726372 & -2.559085 & -4.295921\end{array}$

Table S30. Structure for $4\left[\mathrm{H}_{2} \mathrm{O}, \mathrm{OH}\right]$. $E=-5762.91272502$ Hartrees, $G=-5762.350361$ Hartrees

\begin{tabular}{lrrr}
\hline Atom & \multicolumn{1}{c}{$\mathrm{X}$} & \multicolumn{1}{c}{$\mathrm{Y}$} & \multicolumn{1}{c}{$\mathrm{Z}$} \\
\hline \hline $\mathrm{C}$ & 5.658135 & 2.779143 & 2.576224 \\
$\mathrm{C}$ & -5.720018 & 2.78371 & -2.47753 \\
$\mathrm{C}$ & 5.648807 & 2.811333 & -2.5617 \\
$\mathrm{C}$ & -5.680864 & 2.801012 & 2.485716 \\
$\mathrm{C}$ & 4.318361 & 3.017469 & 2.9168 \\
$\mathrm{C}$ & -4.378072 & 3.075865 & -2.764017 \\
$\mathrm{C}$ & 4.307602 & 3.072373 & -2.879293 \\
$\mathrm{C}$ & -4.344134 & 3.082209 & 2.804633 \\
$\mathrm{C}$ & 4.01477 & 4.083905 & 3.776405 \\
$\mathrm{C}$ & -4.083042 & 4.109165 & -3.66569 \\
$\mathrm{C}$ & 4.012746 & 4.113661 & -3.772267 \\
$\mathrm{C}$ & -4.06367 & 4.115817 & 3.710336 \\
$\mathrm{C}$ & 5.027421 & 4.902742 & 4.270722 \\
$\mathrm{C}$ & -5.107251 & 4.853299 & -4.243353 \\
$\mathrm{C}$ & 5.031879 & 4.896539 & -4.30763
\end{tabular}




\begin{tabular}{|c|c|c|c|}
\hline C & -5.095747 & 4.870379 & 4.261282 \\
\hline C & 6.375192 & 4.674102 & 3.930543 \\
\hline C & -6.456685 & 4.590824 & -3.936414 \\
\hline C & 6.379894 & 4.659735 & -3.973593 \\
\hline C & -6.440088 & 4.611248 & 3.928647 \\
\hline C & 6.669146 & 3.591594 & 3.079269 \\
\hline C & -6.741741 & 3.527916 & -3.058917 \\
\hline C & 6.66666 & 3.589479 & -3.104512 \\
\hline C & -6.711461 & 3.550369 & 3.044001 \\
\hline C & -5.645841 & -2.871252 & -2.476937 \\
\hline C & 5.693032 & -2.742498 & 2.571357 \\
\hline C & -5.680674 & -2.859187 & 2.495311 \\
\hline C & 5.699745 & -2.707532 & -2.590624 \\
\hline C & -4.30679 & -3.13836 & -2.798304 \\
\hline C & 4.356509 & -3.026184 & 2.890945 \\
\hline C & -4.334775 & -3.137949 & 2.776537 \\
\hline C & 4.362653 & -2.967263 & -2.929485 \\
\hline C & -4.014876 & -4.168053 & -3.704431 \\
\hline C & 4.080928 & -4.069285 & 3.788428 \\
\hline C & -4.025122 & -4.166564 & 3.678463 \\
\hline C & 4.076927 & -4.035852 & -3.792875 \\
\hline C & -5.038215 & -4.934931 & -4.253115 \\
\hline C & 5.115103 & -4.833732 & 4.321724 \\
\hline C & -5.039301 & -4.920585 & 4.260985 \\
\hline C & 5.102679 & -4.837251 & -4.288873 \\
\hline C & -6.384268 & -4.692192 & -3.91688 \\
\hline C & 6.458591 & -4.576456 & 3.983879 \\
\hline C & -6.39235 & -4.671978 & 3.958745 \\
\hline C & 6.44699 & -4.590194 & -3.947637 \\
\hline C & -6.667844 & -3.633734 & -3.033271 \\
\hline C & 6.725853 & -3.501515 & 3.113713 \\
\hline C & -6.692597 & -3.613657 & 3.080889 \\
\hline C & 6.723797 & -3.503144 & -3.096188 \\
\hline C & 3.227355 & 2.262568 & -2.240607 \\
\hline C & 3.238498 & 2.187404 & 2.314089 \\
\hline C & 3.263697 & -2.236953 & 2.247724 \\
\hline C & -3.246221 & -2.368598 & 2.115323 \\
\hline C & -3.221161 & -2.33149 & -2.169093 \\
\hline C & -3.276893 & 2.316913 & -2.10705 \\
\hline C & 3.268572 & -2.159611 & -2.3173 \\
\hline C & -3.249947 & 2.284487 & 2.176982 \\
\hline $\mathrm{H}$ & 5.886658 & 1.956207 & 1.902507 \\
\hline $\mathrm{H}$ & -5.94133 & 1.974496 & -1.784784 \\
\hline
\end{tabular}




\begin{tabular}{lrrr} 
H & 5.873992 & 1.999661 & -1.873514 \\
$\mathrm{H}$ & -5.890613 & 1.990023 & 1.791775 \\
$\mathrm{H}$ & 2.973127 & 4.263874 & 4.034958 \\
$\mathrm{H}$ & -3.041831 & 4.342538 & -3.877074 \\
$\mathrm{H}$ & 2.970723 & 4.30674 & -4.020383 \\
$\mathrm{H}$ & -3.029074 & 4.313343 & 3.983174 \\
$\mathrm{H}$ & 4.770165 & 5.73133 & 4.928959 \\
$\mathrm{H}$ & -4.8586 & 5.662107 & -4.928951 \\
$\mathrm{H}$ & 4.778835 & 5.709611 & -4.98649 \\
$\mathrm{H}$ & -4.858081 & 5.671398 & 4.959668 \\
$\mathrm{H}$ & 7.703504 & 3.391233 & 2.802733 \\
$\mathrm{H}$ & -7.777503 & 3.292971 & -2.817702 \\
$\mathrm{H}$ & 7.701522 & 3.374085 & -2.841323 \\
$\mathrm{H}$ & -7.74305 & 3.320334 & 2.781162 \\
$\mathrm{H}$ & -5.864545 & -2.064772 & -1.78059 \\
$\mathrm{H}$ & 5.901919 & -1.927112 & 1.882569 \\
$\mathrm{H}$ & -5.91327 & -2.052916 & 1.802811 \\
$\mathrm{H}$ & 5.914862 & -1.880208 & -1.918004 \\
$\mathrm{H}$ & -2.973756 & -4.360779 & -3.955903 \\
$\mathrm{H}$ & 3.044624 & -4.29521 & 4.032005 \\
$\mathrm{H}$ & -2.979056 & -4.371184 & 3.895318 \\
$\mathrm{H}$ & 3.043707 & -4.223746 & -4.077979 \\
$\mathrm{H}$ & -4.791855 & -5.736842 & -4.947522 \\
$\mathrm{H}$ & 4.877459 & -5.651405 & 5.000596 \\
$\mathrm{H}$ & -4.780057 & -5.723193 & 4.950021 \\
$\mathrm{H}$ & 4.858826 & -5.664436 & -4.953893 \\
$\mathrm{H}$ & -7.701915 & -3.418154 & -2.767428 \\
$\mathrm{H}$ & 7.756101 & -3.268324 & 2.848288 \\
$\mathrm{H}$ & -7.731904 & -3.390251 & 2.843494 \\
$\mathrm{H}$ & 7.754807 & -3.284989 & -2.821192 \\
$\mathrm{H}$ & 7.175828 & 5.275004 & -4.386263 \\
$\mathrm{H}$ & -7.255715 & 5.180293 & -4.379489 \\
$\mathrm{H}$ & 7.264636 & -5.178767 & 4.395594 \\
$\mathrm{H}$ & 7.246729 & -5.216839 & -4.334617 \\
$\mathrm{H}$ & -7.183838 & -5.268685 & 4.406073 \\
$\mathrm{H}$ & -7.245449 & 5.202926 & 4.357028 \\
$\mathrm{H}$ & -7.183601 & -5.29513 & -4.341288 \\
$\mathrm{H}$ & 7.165696 & 5.314206 & 4.315048 \\
$\mathrm{H}$ & -2.17959 & 0.016332 & -2.700327 \\
$\mathrm{H}$ & 2.161678 & 2.743912 & 0.068374 \\
& 0.860841 & -2.368414 & 4.28241 \\
\hline & 2.166388 & -0.078142 & 2.720695 \\
\hline
\end{tabular}




\begin{tabular}{|c|c|c|c|}
\hline $\mathrm{H}$ & -0.832361 & 4.28657 & 2.401881 \\
\hline 0 & 1.026094 & -0.063324 & 1.551262 \\
\hline 0 & 3.58781 & -1.443103 & 1.307845 \\
\hline 0 & 3.586691 & -1.279956 & -1.458575 \\
\hline 0 & 3.573163 & 1.33048 & 1.43005 \\
\hline 0 & 2.060415 & 2.419214 & 2.698126 \\
\hline 0 & 0.023294 & 4.088416 & 1.980976 \\
\hline 0 & -2.061904 & 2.653867 & 2.416844 \\
\hline 0 & -2.10006 & 2.623004 & -2.457975 \\
\hline 0 & -3.57535 & 1.287454 & 1.460614 \\
\hline 0 & -3.592431 & 1.412201 & -1.273928 \\
\hline $\mathrm{O}$ & -3.579167 & -1.434908 & 1.312733 \\
\hline 0 & -3.566146 & -1.344794 & -1.436753 \\
\hline $\mathrm{O}$ & -2.027673 & -2.666287 & -2.420398 \\
\hline 0 & -2.063184 & -2.68978 & 2.412569 \\
\hline 0 & -1.017907 & -1.569555 & -0.065503 \\
\hline 0 & -1.356157 & -0.107631 & 2.187878 \\
\hline O & -0.092048 & 1.57097 & 4.051085 \\
\hline $\mathrm{O}$ & -0.003501 & -1.969266 & 4.077412 \\
\hline 0 & -0.128783 & 2.021652 & -4.081191 \\
\hline 0 & -1.028363 & 1.546121 & 0.065073 \\
\hline 0 & 1.356968 & 2.203462 & 0.103457 \\
\hline $\mathrm{O}$ & 2.042496 & 2.443538 & -2.642552 \\
\hline O & -0.001634 & 4.045775 & -1.558176 \\
\hline 0 & 3.567842 & 1.469779 & -1.300226 \\
\hline $\mathrm{O}$ & 0.067923 & -4.052741 & 1.530548 \\
\hline 0 & 0.139691 & -4.086681 & -2.013531 \\
\hline $\mathrm{O}$ & 1.387491 & -2.148688 & -0.087699 \\
\hline $\mathrm{O}$ & 2.07994 & -2.446247 & 2.649187 \\
\hline 0 & 2.088837 & -2.462188 & -2.661998 \\
\hline 0 & 0.003102 & -1.517984 & -4.052842 \\
\hline 0 & -1.37574 & 0.072893 & -2.160663 \\
\hline $\mathrm{O}$ & 1.020455 & 0.070329 & -1.547777 \\
\hline $\mathrm{Zr}$ & -2.397595 & -0.022796 & 0.007099 \\
\hline $\mathrm{Zr}$ & 0.026174 & 1.748426 & 2.039271 \\
\hline $\mathrm{Zr}$ & -0.019843 & -2.041163 & 1.748178 \\
\hline $\mathrm{Zr}$ & 0.018757 & -1.750596 & -2.046719 \\
\hline $\mathrm{Zr}$ & 2.406614 & 0.015815 & 0.000376 \\
\hline $\mathrm{Zr}$ & -0.016553 & 2.035326 & -1.751969 \\
\hline $\mathrm{Zn}$ & 0.035129 & -4.302968 & -0.20526 \\
\hline $\mathrm{Zn}$ & 0.019057 & -0.162588 & 4.294752 \\
\hline $\mathrm{Zn}$ & -0.005963 & 0.216789 & -4.304588 \\
\hline $\mathrm{Zn}$ & -0.063709 & 4.291774 & 0.176445 \\
\hline
\end{tabular}




$\begin{array}{lrrr}H & 1.044554 & -4.248662 & -2.334452 \\ \mathrm{H} & -1.034302 & 2.338864 & -4.245909\end{array}$

Table S31. Structure for $4\left[\mathrm{H}_{2} \mathrm{O}, \mathrm{OH}\right]-4 \mathrm{Hy}$. $E=-6068.67134419$ Hartrees, $G=-6068.023786$ Hartrees

\begin{tabular}{|c|c|c|c|}
\hline Atom & $X$ & $\mathrm{Y}$ & Z \\
\hline$C$ & -5.688208 & -2.084719 & 3.101009 \\
\hline$C$ & 5.172188 & -3.472143 & -2.825969 \\
\hline$C$ & -6.101986 & -2.248198 & -2.01777 \\
\hline$C$ & 5.536714 & -3.359017 & 2.122784 \\
\hline C & -4.358719 & -2.460518 & 3.34343 \\
\hline$C$ & 3.787644 & -3.621793 & -2.996265 \\
\hline C & -4.827177 & -2.663462 & -2.430532 \\
\hline C & 4.207976 & -3.482891 & 2.554933 \\
\hline C & -4.102798 & -3.531731 & 4.212515 \\
\hline$C$ & 3.310234 & -4.638835 & -3.836157 \\
\hline$C$ & -4.720286 & -3.753259 & -3.307705 \\
\hline C & 3.892528 & -4.455956 & 3.514582 \\
\hline C & -5.154123 & -4.221111 & 4.812341 \\
\hline C & 4.197626 & -5.505785 & -4.46656 \\
\hline$C$ & -5.858215 & -4.432342 & -3.734459 \\
\hline C & 4.8787 & -5.305472 & 4.008417 \\
\hline$C$ & -6.49261 & -3.853961 & 4.571227 \\
\hline C & 5.588017 & -5.386 & -4.275483 \\
\hline$C$ & -7.141018 & -4.03993 & -3.304083 \\
\hline C & 6.211478 & -5.204702 & 3.562775 \\
\hline C & -6.736585 & -2.767616 & 3.70912 \\
\hline C & 6.056754 & -4.339073 & -3.459853 \\
\hline$C$ & -7.238742 & -2.922851 & -2.452277 \\
\hline C & 6.522574 & -4.203035 & 2.623846 \\
\hline$C$ & 5.708749 & 2.154567 & -3.012824 \\
\hline$C$ & -5.127623 & 3.405191 & 2.910221 \\
\hline C & 6.147905 & 2.265016 & 1.939039 \\
\hline C & -5.559481 & 3.240104 & -2.231262 \\
\hline C & 4.384519 & 2.559664 & -3.237148 \\
\hline$C$ & -3.746626 & 3.547923 & 3.114113 \\
\hline $\mathrm{C}$ & 4.867356 & 2.697725 & 2.315109 \\
\hline C & -4.234226 & 3.342252 & -2.682562 \\
\hline C & 4.132438 & 3.591829 & -4.152191 \\
\hline $\mathrm{C}$ & -3.287842 & 4.576714 & 3.951015 \\
\hline C & 4.745197 & 3.776738 & 3.202788 \\
\hline C & -3.906401 & 4.350482 & -3.601613 \\
\hline
\end{tabular}




$\begin{array}{lrrr}\text { C } & 5.184341 & 4.226925 & -4.805325 \\ \mathrm{C} & -4.186508 & 5.463791 & 4.537347 \\ \mathrm{C} & 5.878953 & 4.428822 & 3.677992 \\ \mathrm{C} & -4.8768 & 5.247216 & -4.042797 \\ \mathrm{C} & 6.519269 & 3.845859 & -4.567536 \\ \mathrm{C} & -5.572979 & 5.347733 & 4.314829 \\ \mathrm{C} & 7.168068 & 4.024923 & 3.27932 \\ \mathrm{C} & -6.207537 & 5.158646 & -3.589021 \\ \mathrm{C} & 6.758197 & 2.785385 & -3.673215 \\ \mathrm{C} & -6.024786 & 4.287061 & 3.505532 \\ \mathrm{C} & 7.279721 & 2.91799 & 2.417316 \\ \mathrm{C} & -6.529556 & 4.130724 & -2.681861 \\ \mathrm{C} & -3.617288 & -1.961439 & -1.906619 \\ \mathrm{C} & -3.24852 & -1.770018 & 2.629844 \\ \mathrm{C} & -2.801547 & 2.626636 & 2.41428 \\ \mathrm{C} & 3.651784 & 2.036094 & 1.767328 \\ \mathrm{C} & 3.27313 & 1.893355 & -2.49682 \\ \mathrm{C} & 2.832211 & -2.729705 & -2.281243 \\ \mathrm{C} & -3.187422 & 2.434291 & -2.130025 \\ \mathrm{C} & 3.158695 & -2.585483 & 1.988083 \\ \mathrm{H} & -5.878723 & -1.256871 & 2.421469 \\ \mathrm{H} & 5.534134 & -2.675591 & -2.179327 \\ \mathrm{H} & -6.177459 & -1.400416 & -1.340777 \\ \mathrm{H} & 5.77253 & -2.596203 & 1.383932 \\ \mathrm{H} & -3.068237 & -3.817082 & 4.393173 \\ \mathrm{H} & 2.235457 & -4.75073 & -3.961194 \\ \mathrm{H} & -3.730172 & -4.078877 & -3.628017 \\ \mathrm{H} & 2.8669 & -4.531528 & 3.870164 \\ \mathrm{H} & -4.935069 & -5.055024 & 5.477974 \\ \mathrm{H} & 3.808563 & -6.29918 & -5.103504 \\ \mathrm{H} & -5.751854 & -5.286403 & -4.402141 \\ \mathrm{H} & 4.61375 & -6.058853 & 4.748995 \\ \mathrm{H} & -7.762716 & -2.460353 & 3.509819 \\ \mathrm{H} & 7.128392 & -4.214042 & -3.309176 \\ \mathrm{H} & -8.219353 & -2.58818 & -2.116657 \\ \mathrm{H} & 7.547914 & -4.09725 & 2.271423 \\ \mathrm{H} & 5.89159 & 1.342107 & -2.312882 \\ \mathrm{H} & -5.477533 & 2.600632 & 2.267482 \\ \mathrm{H} & 6.23218 & 1.418458 & 1.260839 \\ \mathrm{H} & -5.804572 & 2.460636 & -1.513325 \\ \mathrm{H} & 3.10146 & 3.879194 & -4.353908 \\ \mathrm{H} & -2.216348 & 4.691069 & 4.103559 \\ \mathrm{H} & 3.748913 & 4.116403 & 3.481585 \\ & & & \\ & & & \end{array}$




\begin{tabular}{|c|c|c|c|}
\hline $\mathrm{H}$ & -2.882362 & 4.418875 & -3.963808 \\
\hline $\mathrm{H}$ & 4.969635 & 5.028491 & -5.510327 \\
\hline $\mathrm{H}$ & -3.807778 & 6.267623 & 5.167235 \\
\hline $\mathrm{H}$ & 5.765502 & 5.2763 & 4.352799 \\
\hline $\mathrm{H}$ & -4.599976 & 6.027423 & -4.750605 \\
\hline $\mathrm{H}$ & 7.78106 & 2.461975 & -3.484658 \\
\hline $\mathrm{H}$ & -7.092655 & 4.162588 & 3.329936 \\
\hline $\mathrm{H}$ & 8.266284 & 2.574417 & 2.108997 \\
\hline $\mathrm{H}$ & -7.551953 & 4.036614 & -2.317841 \\
\hline $\mathrm{H}$ & -8.02914 & -4.574307 & -3.632316 \\
\hline $\mathrm{H}$ & 6.280281 & -6.070795 & -4.759669 \\
\hline $\mathrm{H}$ & -6.273229 & 6.045638 & 4.767483 \\
\hline $\mathrm{H}$ & -6.963694 & 5.86077 & -3.931988 \\
\hline $\mathrm{H}$ & 8.052832 & 4.542794 & 3.641829 \\
\hline $\mathrm{H}$ & 6.980474 & -5.871531 & 3.945568 \\
\hline $\mathrm{H}$ & 7.341113 & 4.3444 & -5.075829 \\
\hline $\mathrm{H}$ & -7.313639 & -4.391869 & 5.039719 \\
\hline $\mathrm{H}$ & 1.795235 & -0.302776 & -2.915897 \\
\hline $\mathrm{H}$ & -2.436219 & -2.489025 & 0.29756 \\
\hline $\mathrm{H}$ & -0.323005 & 2.383138 & 4.235862 \\
\hline $\mathrm{H}$ & 2.431087 & -0.150694 & 2.492743 \\
\hline $\mathrm{H}$ & -1.768692 & 2.95114 & 0.016531 \\
\hline $\mathrm{H}$ & 0.584528 & -4.299115 & 2.346406 \\
\hline $\mathrm{O}$ & -0.872894 & 0.184399 & 1.596305 \\
\hline $\mathrm{O}$ & -3.292669 & 1.843598 & 1.540667 \\
\hline $\mathrm{O}$ & -3.540448 & 1.608265 & -1.231533 \\
\hline $\mathrm{O}$ & -3.563797 & -0.899869 & 1.751239 \\
\hline $\mathrm{O}$ & -2.079979 & -2.130142 & 2.924869 \\
\hline $\mathrm{O}$ & -0.312141 & -4.004025 & 2.109673 \\
\hline $\mathrm{O}$ & 1.96462 & -2.829684 & 2.318735 \\
\hline $\mathrm{O}$ & 1.607894 & -2.897864 & -2.539406 \\
\hline $\mathrm{O}$ & 3.540221 & -1.63656 & 1.231 \\
\hline $\mathrm{O}$ & 3.31963 & -1.864234 & -1.489204 \\
\hline $\mathrm{O}$ & 3.819794 & 1.108364 & 0.9213 \\
\hline $\mathrm{O}$ & 3.563464 & 0.880772 & -1.799831 \\
\hline $\mathrm{O}$ & 2.132264 & 2.440696 & -2.604404 \\
\hline $\mathrm{O}$ & 2.549861 & 2.447202 & 2.234521 \\
\hline $\mathrm{O}$ & 1.235726 & 1.476719 & -0.167219 \\
\hline $\mathrm{O}$ & 1.567633 & -0.019698 & 2.065657 \\
\hline $\mathrm{O}$ & 0.269683 & -1.489165 & 4.079243 \\
\hline $\mathrm{O}$ & 0.546371 & 2.035654 & 3.974797 \\
\hline $\mathrm{O}$ & -0.460336 & -1.911545 & -3.900903 \\
\hline $\mathrm{O}$ & 0.866476 & -1.651686 & 0.044627 \\
\hline
\end{tabular}




$\begin{array}{lrrr}\mathrm{O} & -1.582222 & -2.024213 & 0.254442 \\ \mathrm{O} & -2.510858 & -2.271779 & -2.451518 \\ \mathrm{O} & -0.609084 & -4.049173 & -1.464743 \\ \mathrm{O} & -3.789905 & -1.133546 & -0.970573 \\ \mathrm{O} & 0.478086 & 4.021271 & 1.420385 \\ \mathrm{O} & 0.154332 & 3.986172 & -2.025051 \\ \mathrm{O} & -1.154518 & 2.205266 & -0.038286 \\ \mathrm{O} & -1.579211 & 2.711163 & 2.72234 \\ \mathrm{O} & -2.016193 & 2.596804 & -2.574542 \\ \mathrm{O} & -0.092417 & 1.365323 & -3.986133 \\ \mathrm{O} & 1.205422 & -0.25826 & -2.150579 \\ \mathrm{O} & -1.218978 & -0.008392 & -1.561903 \\ \mathrm{Zr} & 2.366421 & -0.2692 & -0.188785 \\ \mathrm{Zr} & -0.041663 & -1.701283 & 2.127855 \\ \mathrm{Zr} & 0.358969 & 2.053959 & 1.686271 \\ \mathrm{Zr} & 0.032091 & 1.721173 & -2.052758 \\ \mathrm{Zr} & -2.367062 & 0.243691 & 0.195271 \\ \mathrm{Zr} & -0.295377 & -2.063095 & -1.687172 \\ \mathrm{Zn} & 0.90068 & 4.293225 & -0.301038 \\ \mathrm{O} & 2.686231 & 4.799482 & -1.230697 \\ \mathrm{H} & 2.550763 & 4.216298 & -2.016291 \\ \mathrm{H} & 3.489572 & 4.466957 & -0.799169 \\ \mathrm{Zn} & 0.785397 & 0.206898 & 4.31641 \\ \mathrm{Zn} & -1.199846 & -0.090606 & -3.917181 \\ \mathrm{Zn} & -0.647746 & -4.254862 & 0.324469 \\ \mathrm{O} & -2.979516 & -0.818647 & -4.672486 \\ \mathrm{H} & -2.871523 & -1.045669 & -5.609273 \\ \mathrm{O} & 2.901389 & 0.777318 & 4.500349 \\ \mathrm{H} & 3.380492 & 0.881288 & 5.334866 \\ \mathrm{O} & -2.89549 & -4.468655 & -0.190755 \\ \mathrm{H} & -3.547282 & -5.183964 & -0.190831 \\ \mathrm{H} & 0.436553 & -1.951845 & -4.264277 \\ \mathrm{H} & -2.897147 & -1.673144 & -4.180831 \\ \mathrm{H} & 2.774644 & 1.679631 & 4.131661 \\ \mathrm{H} & -2.477705 & -4.435781 & -1.087278 \\ & -0.765146 & 4.276492 & -2.129688\end{array}$


Table S32. Structure for $\mathrm{H}_{2} \mathrm{O} / \mu \mathrm{OH}$.

$E=-5310.95770663$ Hartrees, $G=-5310.342720$ Hartrees

\begin{tabular}{|c|c|c|c|}
\hline Atom & $x$ & $Y$ & $Z$ \\
\hline$C$ & 5.674275 & -2.577966 & -2.481084 \\
\hline $\mathrm{C}$ & -5.69427 & -2.546232 & 2.594282 \\
\hline$C$ & 5.674278 & -2.443926 & 2.655414 \\
\hline$C$ & -5.663593 & -2.723784 & -2.36585 \\
\hline$C$ & 4.336784 & -2.842041 & -2.810889 \\
\hline$C$ & -4.348587 & -2.814121 & 2.887058 \\
\hline$C$ & 4.336457 & -2.709636 & 2.984221 \\
\hline$C$ & -4.324366 & -3.000136 & -2.678377 \\
\hline C & 4.043593 & -3.939185 & -3.634973 \\
\hline$C$ & -4.040406 & -3.814072 & 3.821286 \\
\hline$C$ & 4.055064 & -3.724752 & 3.910697 \\
\hline $\mathrm{C}$ & -4.033879 & -4.059196 & -3.550939 \\
\hline$C$ & 5.064504 & -4.762284 & -4.104598 \\
\hline C & -5.055203 & -4.550596 & 4.42479 \\
\hline $\mathrm{C}$ & 5.083884 & -4.478346 & 4.469082 \\
\hline C & -5.058354 & -4.842785 & -4.074894 \\
\hline C & 6.410211 & -4.507712 & -3.774814 \\
\hline$C$ & -6.408029 & -4.313311 & 4.112349 \\
\hline C & 6.428563 & -4.237448 & 4.124668 \\
\hline C & -6.404935 & -4.588117 & -3.747923 \\
\hline C & 6.69346 & -3.394998 & -2.959629 \\
\hline C & -6.706553 & -3.282587 & 3.201558 \\
\hline C & 6.701758 & -3.192791 & 3.220749 \\
\hline C & -6.686672 & -3.50229 & -2.89745 \\
\hline$C$ & -5.683685 & 3.106152 & 2.410672 \\
\hline$C$ & 5.647092 & 2.94084 & -2.654912 \\
\hline C & -5.727064 & 2.932762 & -2.558664 \\
\hline C & 5.663223 & 3.07302 & 2.505711 \\
\hline C & -4.347122 & 3.398601 & 2.720299 \\
\hline$C$ & 4.306866 & 3.199162 & -2.980637 \\
\hline C & -4.384943 & 3.217337 & -2.851459 \\
\hline C & 4.323881 & 3.328759 & 2.838605 \\
\hline$C$ & -4.065251 & 4.460291 & 3.591964 \\
\hline$C$ & 4.01804 & 4.209536 & -3.91074 \\
\hline C & -4.08839 & 4.219713 & -3.786845 \\
\hline C & 4.027654 & 4.421494 & 3.667569 \\
\hline $\mathrm{C}$ & -5.096197 & 5.232957 & 4.117809 \\
\hline$C$ & 5.042629 & 4.967806 & -4.470695 \\
\hline $\mathrm{C}$ & -5.111987 & 4.943045 & -4.391375 \\
\hline
\end{tabular}




\begin{tabular}{|c|c|c|c|}
\hline C & 5.045209 & 5.24987 & 4.135326 \\
\hline C & -6.440019 & 4.964436 & 3.79243 \\
\hline C & 6.38951 & 4.736651 & -4.127525 \\
\hline C & -6.461627 & 4.689242 & -4.078389 \\
\hline C & 6.391609 & 5.006967 & 3.799389 \\
\hline C & -6.71322 & 3.87485 & 2.94409 \\
\hline C & 6.67036 & 3.693499 & -3.2236 \\
\hline C & -6.748423 & 3.656605 & -3.166127 \\
\hline C & 6.679131 & 3.896099 & 2.98295 \\
\hline C & 3.248036 & -1.93265 & 2.321358 \\
\hline C & 3.248514 & -2.005709 & -2.232816 \\
\hline C & 3.224274 & 2.419102 & -2.309503 \\
\hline C & -3.286137 & 2.482426 & -2.168175 \\
\hline C & -3.254067 & 2.584213 & 2.11496 \\
\hline C & -3.258809 & -2.065184 & 2.203397 \\
\hline C & 3.238337 & 2.490017 & 2.25522 \\
\hline C & -3.238736 & -2.170102 & -2.079289 \\
\hline $\mathrm{H}$ & 5.894486 & -1.729752 & -1.837287 \\
\hline $\mathrm{H}$ & -5.927392 & -1.760686 & 1.879052 \\
\hline $\mathrm{H}$ & 5.888147 & -1.649878 & 1.94404 \\
\hline $\mathrm{H}$ & -5.88407 & -1.891885 & -1.700882 \\
\hline $\mathrm{H}$ & 3.003456 & -4.146745 & -3.879545 \\
\hline $\mathrm{H}$ & -2.995238 & -4.009037 & 4.050739 \\
\hline $\mathrm{H}$ & 3.01747 & -3.930108 & 4.16637 \\
\hline $\mathrm{H}$ & -2.994173 & -4.262401 & -3.799968 \\
\hline $\mathrm{H}$ & 4.816134 & -5.615669 & -4.734038 \\
\hline $\mathrm{H}$ & -4.79684 & -5.330116 & 5.14007 \\
\hline $\mathrm{H}$ & 4.842307 & -5.270852 & 5.17590 \\
\hline $\mathrm{H}$ & -4.81241 & -5.664698 & -4.74541 \\
\hline $\mathrm{H}$ & 7.726102 & -3.172657 & -2.6938 \\
\hline $\mathrm{H}$ & -7.745404 & -3.065334 & 2.95685 \\
\hline $\mathrm{H}$ & 7.733461 & -2.974558 & 2.94817 \\
\hline $\mathrm{H}$ & -7.719941 & -3.276538 & -2.637702 \\
\hline $\mathrm{H}$ & -5.894773 & 2.273991 & 1.743472 \\
\hline $\mathrm{H}$ & 5.866074 & 2.151353 & -1.939998 \\
\hline $\mathrm{H}$ & -5.949541 & 2.14659 & -1.840804 \\
\hline $\mathrm{H}$ & 5.886921 & 2.22842 & 1.85818 \\
\hline $\mathrm{H}$ & -3.028979 & 4.66848 & 3.849489 \\
\hline $\mathrm{H}$ & 2.978606 & 4.410525 & -4.161364 \\
\hline $\mathrm{H}$ & -3.047324 & 4.422091 & -4.02718 \\
\hline $\mathrm{H}$ & 2.988381 & 4.624944 & 3.91564 \\
\hline $\mathrm{H}$ & -4.858451 & 6.057867 & 4.78778 \\
\hline $\mathrm{H}$ & 4.795366 & 5.759845 & -5.17618 \\
\hline
\end{tabular}




\begin{tabular}{|c|c|c|c|}
\hline $\mathrm{H}$ & -4.863868 & 5.723384 & -5.109378 \\
\hline $\mathrm{H}$ & 4.79277 & 6.100871 & 4.76636 \\
\hline $\mathrm{H}$ & -7.745233 & 3.637817 & 2.689179 \\
\hline $\mathrm{H}$ & 7.703666 & 3.48164 & -2.951777 \\
\hline $\mathrm{H}$ & -7.784507 & 3.428113 & -2.92034 \\
\hline $\mathrm{H}$ & 7.711785 & 3.682188 & 2.710474 \\
\hline $\mathrm{H}$ & 7.231735 & -4.829822 & 4.556142 \\
\hline $\mathrm{H}$ & -7.199887 & -4.895563 & 4.577468 \\
\hline $\mathrm{H}$ & 7.188099 & 5.334651 & -4.559808 \\
\hline $\mathrm{H}$ & 7.184853 & 5.656357 & 4.161777 \\
\hline $\mathrm{H}$ & -7.260166 & 5.261712 & -4.544184 \\
\hline $\mathrm{H}$ & -7.204618 & -5.202248 & -4.154863 \\
\hline $\mathrm{H}$ & -7.245011 & 5.571249 & 4.200003 \\
\hline $\mathrm{H}$ & 7.206912 & -5.15099 & -4.140667 \\
\hline $\mathrm{H}$ & -1.972841 & 0.206373 & 2.844547 \\
\hline $\mathrm{H}$ & 0.952474 & 1.734167 & -4.260946 \\
\hline $\mathrm{H}$ & -0.003556 & 0.479171 & -4.085935 \\
\hline $\mathrm{H}$ & 0.738607 & 2.028309 & 4.356569 \\
\hline $\mathrm{H}$ & -0.044833 & 4.300872 & 0.236115 \\
\hline $\mathrm{H}$ & -0.807872 & 4.552583 & -1.756429 \\
\hline $\mathrm{H}$ & -0.968695 & 4.524351 & 1.510393 \\
\hline $\mathrm{H}$ & -1.98877 & 0.176184 & -2.81776 \\
\hline $\mathrm{H}$ & 1.949268 & 3.087477 & -0.025175 \\
\hline $\mathrm{H}$ & 0.565653 & -1.608784 & -4.487553 \\
\hline $\mathrm{H}$ & 0.043436 & 0.020878 & 4.089405 \\
\hline $\mathrm{H}$ & 0.97579 & -1.243887 & 4.350683 \\
\hline $\mathrm{O}$ & 1.068177 & 0.265992 & -1.508133 \\
\hline $\mathrm{O}$ & 3.584 & 1.615297 & -1.395853 \\
\hline 0 & 3.5841 & 1.610654 & 1.412222 \\
\hline 0 & 3.60471 & -1.142072 & -1.374215 \\
\hline $\mathrm{O}$ & 2.079441 & -2.193905 & -2.672641 \\
\hline $\mathrm{O}$ & 0.163248 & -3.694479 & -1.532219 \\
\hline 0 & -2.046314 & -2.613077 & -2.1756 \\
\hline 0 & -2.063604 & -2.338073 & 2.514308 \\
\hline $\mathrm{O}$ & -3.597439 & -1.135223 & -1.446125 \\
\hline 0 & -3.620345 & -1.207174 & 1.327959 \\
\hline 0 & -3.631043 & 1.59422 & -1.32734 \\
\hline $\mathrm{O}$ & -3.609309 & 1.597791 & 1.3952 \\
\hline $\mathrm{O}$ & -2.05661 & 2.926704 & 2.356762 \\
\hline $\mathrm{O}$ & -2.092403 & 2.793943 & -2.466851 \\
\hline $\mathrm{O}$ & -1.067176 & 1.677897 & 0.021786 \\
\hline $\mathrm{O}$ & -1.391203 & 0.199397 & -2.056334 \\
\hline $\mathrm{O}$ & -0.091437 & -1.150068 & -3.945726 \\
\hline
\end{tabular}




$\begin{array}{lrrr}\mathrm{O} & 0.044052 & 1.494957 & -4.008978 \\ \mathrm{O} & 0.090898 & -0.999109 & 4.035348 \\ \mathrm{O} & -1.134028 & -1.391602 & 0.001001 \\ \mathrm{O} & 1.422709 & -1.754558 & 0.025266 \\ \mathrm{O} & 2.048667 & -2.200749 & 2.670868 \\ \mathrm{O} & -0.065227 & -3.682146 & 1.376676 \\ \mathrm{O} & 3.604993 & -1.08066 & 1.464185 \\ \mathrm{O} & -0.02412 & 4.130415 & -1.375884 \\ \mathrm{O} & -0.071689 & 4.249274 & 1.256611 \\ \mathrm{O} & 1.367109 & 2.314882 & 0.012938 \\ \mathrm{O} & 2.026096 & 2.632944 & -2.680032 \\ \mathrm{O} & 2.042687 & 2.744207 & 2.616689 \\ \mathrm{O} & -0.037135 & 1.63792 & 3.927737 \\ \mathrm{O} & -1.384316 & 0.212981 & 2.075574 \\ \mathrm{O} & 1.058681 & 0.251249 & 1.531527 \\ \mathrm{Zr} & -2.503684 & 0.219009 & 0.009709 \\ \mathrm{Zr} & 0.093416 & -1.553445 & -1.891516 \\ \mathrm{Zr} & -0.03483 & 2.054276 & -1.761635 \\ \mathrm{Zr} & 0.019553 & 2.008806 & 1.854812 \\ \mathrm{Zr} & 2.477845 & 0.212173 & 0.002467 \\ \mathrm{Zr} & 0.03422 & -1.584629 & 1.797464 \\ \mathrm{Zn} & 1.343769 & -3.676698 & -0.002694 \\ \mathrm{Zn} & -1.239362 & -3.472684 & -0.190511\end{array}$

Table S32. Structure for $2\left[\mathrm{H}_{2} \mathrm{O} / \mu \mathrm{OH}\right]$.

$E=-5762.89579685$ Hartrees, $G=-5762.330234$ Hartrees

\begin{tabular}{lrrr}
\hline Atom & \multicolumn{1}{c}{$\mathrm{X}$} & \multicolumn{1}{c}{$\mathrm{Y}$} & \multicolumn{1}{c}{$\mathrm{Z}$} \\
\hline \hline $\mathrm{C}$ & 5.654167 & 2.825618 & 2.506566 \\
$\mathrm{C}$ & -5.719241 & 2.747772 & -2.55734 \\
$\mathrm{C}$ & 5.649667 & 2.725045 & -2.630598 \\
$\mathrm{C}$ & -5.684557 & 2.893152 & 2.403908 \\
$\mathrm{C}$ & 4.315143 & 3.078262 & 2.839534 \\
C & -4.375782 & 3.026849 & -2.849776 \\
C & 4.309655 & 2.98354 & -2.9564 \\
C & -4.346863 & 3.176858 & 2.71692 \\
C & 4.015281 & 4.16789 & 3.670978 \\
C & -4.075534 & 4.035026 & -3.777761 \\
C & 4.020264 & 4.002658 & -3.875929 \\
C & -4.0629 & 4.232291 & 3.595825 \\
C & 5.030947 & 4.994992 & 4.144931 \\
C & -5.096063 & 4.768415 & -4.375387 \\
C & 5.043227 & 4.767023 & -4.430418
\end{tabular}




\begin{tabular}{|c|c|c|c|}
\hline C & -5.092252 & 5.005259 & 4.125961 \\
\hline C & 6.378041 & 4.751941 & 3.81214 \\
\hline C & -6.446876 & 4.51968 & -4.06314 \\
\hline c & 6.389914 & 4.533238 & -4.088964 \\
\hline C & -6.437378 & 4.743319 & 3.798749 \\
\hline C & 6.668158 & 3.646559 & 2.989426 \\
\hline C & -6.737261 & 3.480989 & -3.158792 \\
\hline C & 6.671324 & 3.484642 & -3.192152 \\
\hline C & -6.71246 & 3.661086 & 2.94154 \\
\hline C & -5.669159 & -2.905481 & -2.410589 \\
\hline C & 5.665544 & -2.694347 & 2.644425 \\
\hline C & -5.708483 & -2.764765 & 2.559801 \\
\hline C & 5.677138 & -2.792763 & -2.516889 \\
\hline C & -4.330942 & -3.186572 & -2.723473 \\
\hline C & 4.327451 & -2.964057 & 2.969948 \\
\hline C & -4.364082 & -3.04189 & 2.849418 \\
\hline$\checkmark$ & 4.339264 & -3.055631 & -2.850098 \\
\hline$\checkmark$ & -4.042599 & -4.240604 & -3.602322 \\
\hline C & 4.046687 & -3.982457 & 3.893655 \\
\hline C & -4.059602 & -4.048203 & 3.777959 \\
\hline C & 4.049759 & -4.145004 & -3.685846 \\
\hline C & -5.0687 & -5.016998 & -4.132135 \\
\hline C & 5.077116 & -4.737209 & 4.447612 \\
\hline C & -5.077504 & -4.782611 & 4.378742 \\
\hline C & 5.072552 & -4.963201 & -4.160042 \\
\hline C & -6.414013 & -4.759955 & -3.803661 \\
\hline C & 6.421994 & -4.494428 & 4.104625 \\
\hline C & -6.429208 & -4.536161 & 4.068801 \\
\hline C & 6.417587 & -4.713122 & -3.823898 \\
\hline C & -6.693886 & -3.677838 & -2.947969 \\
\hline C & 6.694629 & -3.443478 & 3.207241 \\
\hline C & -6.724143 & -3.499616 & 3.163592 \\
\hline C & 6.698239 & -3.605624 & -3.000521 \\
\hline C & 3.227391 & 2.194599 & -2.297337 \\
\hline C & 3.231976 & 2.237771 & 2.256992 \\
\hline C & 3.23879 & -2.186658 & 2.305006 \\
\hline C & -3.271275 & -2.294764 & 2.169534 \\
\hline C & -3.242778 & -2.36864 & -2.113988 \\
\hline C & -3.279972 & 2.281081 & -2.172135 \\
\hline C & 3.248421 & -2.228086 & -2.260236 \\
\hline C & -3.255896 & 2.358561 & 2.110992 \\
\hline $\mathrm{H}$ & 5.878108 & 1.978609 & 1.861411 \\
\hline $\mathrm{H}$ & -5.948587 & 1.952254 & -1.851225 \\
\hline
\end{tabular}




\begin{tabular}{|c|c|c|c|}
\hline $\mathrm{H}$ & 5.870822 & 1.925574 & -1.926558 \\
\hline $\mathrm{H}$ & -5.899606 & 2.060999 & 1.737092 \\
\hline $\mathrm{H}$ & 2.973775 & 4.361523 & 3.920481 \\
\hline $\mathrm{H}$ & -3.031301 & 4.23872 & -4.004191 \\
\hline $\mathrm{H}$ & 2.979836 & 4.202177 & -4.124656 \\
\hline $\mathrm{H}$ & -3.023211 & 4.431407 & 3.849784 \\
\hline $\mathrm{H}$ & 4.777701 & 5.841269 & 4.781892 \\
\hline $\mathrm{H}$ & -4.843858 & 5.554717 & -5.085418 \\
\hline $\mathrm{H}$ & 4.795286 & 5.564393 & -5.129684 \\
\hline $\mathrm{H}$ & -4.851624 & 5.8237 & 4.802636 \\
\hline $\mathrm{H}$ & 7.70217 & 3.433277 & 2.72178 \\
\hline $\mathrm{H}$ & -7.774643 & 3.253916 & -2.916096 \\
\hline $\mathrm{H}$ & 7.705579 & 3.270617 & -2.924665 \\
\hline $\mathrm{H}$ & -7.745012 & 3.427087 & 2.686128 \\
\hline $\mathrm{H}$ & -5.886649 & -2.073772 & -1.744173 \\
\hline $\mathrm{H}$ & 5.879435 & -1.894665 & 1.938421 \\
\hline $\mathrm{H}$ & -5.940449 & -1.970159 & 1.85356 \\
\hline $\mathrm{H}$ & 5.894373 & -1.945617 & -1.869668 \\
\hline $\mathrm{H}$ & -3.001961 & -4.435911 & -3.855259 \\
\hline $\mathrm{H}$ & 3.008264 & -4.190213 & 4.143941 \\
\hline $\mathrm{H}$ & -3.014456 & -4.249558 & 4.00218 \\
\hline $\mathrm{H}$ & 3.010314 & -4.346825 & -3.937486 \\
\hline $\mathrm{H}$ & -4.825711 & -5.834932 & -4.808576 \\
\hline $\mathrm{H}$ & 4.836371 & -5.535294 & 5.148659 \\
\hline $\mathrm{H}$ & -4.82276 & -5.567529 & 5.089366 \\
\hline $\mathrm{H}$ & 4.825963 & -5.809802 & -4.799244 \\
\hline $\mathrm{H}$ & -7.727546 & -3.448028 & -2.69323 \\
\hline $\mathrm{H}$ & 7.726801 & -3.220608 & 2.93905 \\
\hline $\mathrm{H}$ & -7.762507 & -3.274898 & 2.923052 \\
\hline $\mathrm{H}$ & 7.730186 & -3.384529 & -2.731205 \\
\hline $\mathrm{H}$ & 7.188797 & 5.133607 & -4.517638 \\
\hline $\mathrm{H}$ & -7.243443 & 5.098926 & -4.524022 \\
\hline $\mathrm{H}$ & 7.225473 & -5.088337 & 4.533634 \\
\hline $\mathrm{H}$ & 7.215102 & -5.353334 & -4.192822 \\
\hline $\mathrm{H}$ & -7.223699 & -5.116393 & 4.532102 \\
\hline $\mathrm{H}$ & -7.240865 & 5.348631 & 4.21115 \\
\hline $\mathrm{H}$ & -7.215418 & -5.368749 & -4.215082 \\
\hline $\mathrm{H}$ & 7.170707 & 5.397879 & 4.181661 \\
\hline $\mathrm{H}$ & -1.983889 & -0.032497 & -2.805969 \\
\hline $\mathrm{H}$ & 0.922849 & -1.473686 & 4.342308 \\
\hline $\mathrm{H}$ & 0.010437 & -0.20006 & 4.073765 \\
\hline $\mathrm{H}$ & 0.680379 & -1.810125 & -4.419969 \\
\hline $\mathrm{H}$ & -1.986443 & 0.024508 & 2.805547 \\
\hline
\end{tabular}




\begin{tabular}{rrrr}
$\mathrm{H}$ & 0.666011 & 1.807579 & 4.421462 \\
$\mathrm{H}$ & 0.012908 & 0.19461 & -4.0737 \\
$\mathrm{H}$ & 0.918758 & 1.472579 & -4.342062 \\
$\mathrm{O}$ & 1.084608 & 0.012182 & 1.629141 \\
$\mathrm{O}$ & 3.598369 & -1.42099 & 1.358412 \\
$\mathrm{O}$ & 3.604285 & -1.370492 & -1.39818 \\
$\mathrm{O}$ & 3.594084 & 1.378657 & 1.399375 \\
$\mathrm{O}$ & 2.058273 & 2.492558 & 2.646373 \\
$\mathrm{O}$ & 0.034462 & 3.926507 & 1.415334 \\
$\mathrm{O}$ & -2.076453 & 2.683532 & 2.386044 \\
$\mathrm{O}$ & -2.092611 & 2.556155 & -2.482117 \\
$\mathrm{O}$ & -3.6411 & 1.407667 & 1.351518 \\
$\mathrm{O}$ & -3.656786 & 1.432148 & -1.289366 \\
$\mathrm{O}$ & -3.651227 & -1.447832 & 1.286252 \\
$\mathrm{O}$ & -3.631833 & -1.420052 & -1.353694 \\
$\mathrm{O}$ & -2.062269 & -2.692063 & -2.386116 \\
$\mathrm{O}$ & -2.082973 & -2.567297 & 2.478236 \\
$\mathrm{O}$ & -1.183239 & -1.628126 & -0.002029 \\
$\mathrm{O}$ & -1.413983 & 0.009816 & 2.025073 \\
$\mathrm{O}$ & -0.070362 & 1.407883 & 3.938607 \\
$\mathrm{O}$ & 0.035789 & -1.22535 & 4.035418 \\
$\mathrm{O}$ & 0.03287 & 1.219888 & -4.03545 \\
$\mathrm{O}$ & -1.191718 & 1.622127 & 0.001598 \\
$\mathrm{O}$ & 1.339365 & 1.934215 & -0.010703 \\
$\mathrm{O}$ & 2.044898 & 2.382073 & -2.711775 \\
$\mathrm{O}$ & 0.030824 & 3.873877 & -1.461592 \\
$\mathrm{O}$ & 3.59136 & 1.42814 & -1.353395 \\
$\mathrm{O}$ & 0.046184 & -3.878457 & 1.461005 \\
$\mathrm{O}$ & 0.051869 & -3.930565 & -1.415323 \\
$\mathrm{O}$ & 1.348993 & -1.934941 & 0.012077 \\
$\mathrm{O}$ & 2.056387 & -2.381612 & 2.716459 \\
$\mathrm{O}$ & 2.075553 & -2.491848 & -2.646615 \\
$\mathrm{O}$ & -0.058937 & -1.414186 & -3.938481 \\
$\mathrm{O}$ & -1.411355 & -0.018122 & -2.025564 \\
$\mathrm{O}$ & 1.08662 & -0.013559 & -1.62802 \\
$\mathrm{Zr}$ & -2.492601 & -0.005539 & -0.000486 \\
$\mathrm{Zr}$ & 0.034889 & 1.83609 & 1.861361 \\
$\mathrm{Zr}$ & 0.038595 & -1.79744 & 1.783676 \\
$\mathrm{Zr}$ & 0.045959 & -1.84078 & -1.861378 \\
$\mathrm{Zr}$ & 2.364668 & 0.000843 & 0.001216 \\
$\mathrm{Zr}$ & 0.031574 & 1.792452 & -1.7831 \\
$\mathrm{Zn}$ & 1.385196 & 3.926112 & -0.013258 \\
$\mathrm{Zn}$ & -1.288641 & 3.81107 & -0.017413 \\
& & & \\
\hline
\end{tabular}




$\begin{array}{lrrr}\mathrm{O} & -3.297419 & 4.181168 & -0.047476 \\ \mathrm{H} & -3.618989 & 4.525716 & 0.806366 \\ \mathrm{O} & 3.431278 & 4.108498 & -0.025892 \\ \mathrm{H} & 3.822639 & 4.399601 & 0.817277 \\ \mathrm{H} & -3.723717 & 3.298435 & -0.131965 \\ \mathrm{H} & 3.679352 & 3.155121 & -0.102073 \\ \mathrm{Zn} & -1.272927 & -3.818049 & 0.01626 \\ \mathrm{Zn} & 1.402204 & -3.926447 & 0.013882 \\ \mathrm{O} & 3.449711 & -4.096036 & 0.025518 \\ \mathrm{H} & 3.842754 & -4.383093 & -0.818162 \\ \mathrm{O} & -3.28095 & -4.193913 & 0.043166 \\ \mathrm{H} & -3.60001 & -4.538002 & -0.811781 \\ \mathrm{H} & 3.69076 & -3.140825 & 0.103115 \\ \mathrm{H} & -3.709841 & -3.312511 & 0.128393\end{array}$

Table S32. Structure for $2\left[\mathrm{H}_{2} \mathrm{O} / \mu \mathrm{OH}\right]-4 \mathrm{Hy}$.

$E=-6068.72220353$ Hartrees, $G=-6068.064577$ Hartrees

\begin{tabular}{lrrr}
\hline Atom & \multicolumn{1}{c}{$\mathrm{X}$} & \multicolumn{1}{c}{$\mathrm{Y}$} & \multicolumn{1}{c}{$\mathrm{Z}$} \\
\hline $\mathrm{C}$ & 5.652107 & 2.876651 & 2.446666 \\
$\mathrm{C}$ & -5.721079 & 2.694531 & -2.615101 \\
$\mathrm{C}$ & 5.647832 & 2.66789 & -2.687338 \\
$\mathrm{C}$ & -5.686598 & 2.944353 & 2.342001 \\
$\mathrm{C}$ & 4.313082 & 3.136566 & 2.774155 \\
$\mathrm{C}$ & -4.377592 & 2.967092 & -2.913239 \\
$\mathrm{C}$ & 4.307932 & 2.919765 & -3.018478 \\
$\mathrm{C}$ & -4.348814 & 3.234386 & 2.648992 \\
$\mathrm{C}$ & 4.013429 & 4.243508 & 3.582419 \\
$\mathrm{C}$ & -4.077054 & 3.955477 & -3.862262 \\
$\mathrm{C}$ & 4.018747 & 3.919353 & -3.959354 \\
$\mathrm{C}$ & -4.064705 & 4.307934 & 3.505549 \\
$\mathrm{C}$ & 5.029255 & 5.080187 & 4.038913 \\
$\mathrm{C}$ & -5.097398 & 4.67629 & -4.475298 \\
$\mathrm{C}$ & 5.041894 & 4.671672 & -4.529756 \\
$\mathrm{C}$ & -5.09395 & 5.092155 & 4.019155 \\
$\mathrm{C}$ & 6.376312 & 4.829915 & 3.711372 \\
$\mathrm{C}$ & -6.448278 & 4.434481 & -4.15793 \\
$\mathrm{C}$ & 6.388514 & 4.444879 & -4.183354 \\
$\mathrm{C}$ & -6.439111 & 4.823627 & 3.697503 \\
C & 6.666241 & 3.707397 & 2.912125 \\
C & -6.738918 & 3.415142 & -3.231906 \\
C & 6.669665 & 3.415349 & -3.26464 \\
C & -6.714381 & 3.723582 & 2.863327
\end{tabular}




$\begin{array}{lrrr}\text { C } & -5.672162 & -2.954398 & -2.349367 \\ \mathrm{C} & 5.662345 & -2.639119 & 2.700749 \\ \mathrm{C} & -5.711697 & -2.709035 & 2.61697 \\ \mathrm{C} & 5.674162 & -2.846237 & -2.457443 \\ \mathrm{C} & -4.333939 & -3.242363 & -2.656149 \\ \mathrm{C} & 4.32422 & -2.901671 & 3.031706 \\ \mathrm{C} & -4.36741 & -2.98025 & 2.912391 \\ \mathrm{C} & 4.336219 & -3.115826 & -2.785098 \\ \mathrm{C} & -4.045803 & -4.314628 & -3.512644 \\ \mathrm{C} & 4.043161 & -3.900333 & 3.976712 \\ \mathrm{C} & -4.063142 & -3.966886 & 3.861961 \\ \mathrm{C} & 4.04655 & -4.222486 & -3.597705 \\ \mathrm{C} & -5.072063 & -5.101845 & -4.025965 \\ \mathrm{C} & 5.073409 & -4.643469 & 4.546489 \\ \mathrm{C} & -5.081218 & -4.688234 & 4.478067 \\ \mathrm{C} & 5.06921 & -5.050687 & -4.054519 \\ \mathrm{C} & -6.417336 & -4.837637 & -3.703086 \\ \mathrm{C} & 6.41835 & -4.408267 & 4.198504 \\ \mathrm{C} & -6.432856 & -4.448108 & 4.162914 \\ \mathrm{C} & 6.414277 & -4.793867 & -3.723636 \\ \mathrm{C} & -6.697034 & -3.737628 & -2.87045 \\ \mathrm{C} & 6.691243 & -3.376509 & 3.279196 \\ \mathrm{C} & -6.727534 & -3.430813 & 3.236044 \\ \mathrm{C} & 6.695117 & -3.669336 & -2.923742 \\ \mathrm{C} & 3.225407 & 2.145612 & -2.343194 \\ \mathrm{C} & 3.230205 & 2.284337 & 2.209294 \\ \mathrm{C} & 3.235715 & -2.138731 & 2.350681 \\ \mathrm{C} & -3.274222 & -2.248072 & 2.217469 \\ \mathrm{C} & -3.246228 & -2.411839 & -2.063957 \\ \mathrm{C} & -3.281773 & 2.235789 & -2.220478 \\ \mathrm{C} & 3.245909 & -2.275958 & -2.212761 \\ \mathrm{C} & -3.258604 & 2.403113 & 2.060478 \\ \mathrm{H} & 5.876189 & 2.020833 & 1.814197 \\ \mathrm{H} & -5.948401 & 1.917985 & -1.888255 \\ \mathrm{H} & 5.866789 & 1.886011 & -1.963996 \\ \mathrm{H} & -5.902167 & 2.10113 & 1.689741 \\ \mathrm{H} & 2.972176 & 4.44742 & 3.824919 \\ \mathrm{H} & -3.033404 & 4.153697 & -4.095979 \\ \mathrm{H} & 2.979464 & 4.113594 & -4.216594 \\ \mathrm{H} & -3.02627 & 4.522187 & 3.750534 \\ \mathrm{H} & 4.775935 & 5.941266 & 4.655905 \\ \mathrm{H} & -4.844879 & 5.447259 & -5.201798 \\ \mathrm{H} & 4.794404 & 5.452132 & -5.24793 \\ & & & \\ & & & \end{array}$




\begin{tabular}{|c|c|c|c|}
\hline $\mathrm{H}$ & -4.852728 & 5.925323 & 4.677323 \\
\hline $\mathrm{H}$ & 7.700171 & 3.488664 & 2.648069 \\
\hline $\mathrm{H}$ & -7.776032 & 3.194439 & -2.98297 \\
\hline $\mathrm{H}$ & 7.70315 & 3.208637 & -2.989608 \\
\hline $\mathrm{H}$ & -7.746305 & 3.487317 & 2.607714 \\
\hline $\mathrm{H}$ & -5.889883 & -2.111529 & -1.697526 \\
\hline $\mathrm{H}$ & 5.874479 & -1.857037 & 1.975609 \\
\hline $\mathrm{H}$ & -5.941252 & -1.933281 & 1.89001 \\
\hline $\mathrm{H}$ & 5.891912 & -1.99034 & -1.822876 \\
\hline $\mathrm{H}$ & -3.006432 & -4.525452 & -3.756548 \\
\hline $\mathrm{H}$ & 3.005796 & -4.102474 & 4.235555 \\
\hline $\mathrm{H}$ & -3.018677 & -4.163278 & 4.093487 \\
\hline $\mathrm{H}$ & 3.007339 & -4.434186 & -3.842251 \\
\hline $\mathrm{H}$ & -4.828752 & -5.93462 & -4.683858 \\
\hline $\mathrm{H}$ & 4.83271 & -5.424514 & 5.26642 \\
\hline $\mathrm{H}$ & -4.826551 & -5.457902 & 5.205167 \\
\hline $\mathrm{H}$ & 4.822255 & -5.912051 & -4.673757 \\
\hline $\mathrm{H}$ & -7.729986 & -3.505122 & -2.61554 \\
\hline $\mathrm{H}$ & 7.722782 & -3.161338 & 3.00345 \\
\hline $\mathrm{H}$ & -7.765516 & -3.212003 & 2.989198 \\
\hline $\mathrm{H}$ & 7.727038 & -3.443221 & -2.65801 \\
\hline $\mathrm{H}$ & 7.187273 & 5.03642 & -4.624155 \\
\hline $\mathrm{H}$ & -7.244507 & 5.004647 & -4.630472 \\
\hline $\mathrm{H}$ & 7.221428 & -4.993669 & 4.639585 \\
\hline $\mathrm{H}$ & 7.211641 & -5.442464 & -4.07848 \\
\hline $\mathrm{H}$ & -7.227292 & -5.018877 & 4.637819 \\
\hline $\mathrm{H}$ & -7.242503 & 5.438234 & 4.096279 \\
\hline $\mathrm{H}$ & -7.218853 & -5.455385 & -4.100882 \\
\hline $\mathrm{H}$ & 7.169053 & 5.483916 & 4.066798 \\
\hline $\mathrm{H}$ & -1.987896 & -0.040685 & -2.802325 \\
\hline $\mathrm{H}$ & 0.977583 & -1.404407 & 4.357293 \\
\hline $\mathrm{H}$ & 0.014125 & -0.162972 & 4.114252 \\
\hline $\mathrm{H}$ & 0.535796 & -1.933608 & -4.484703 \\
\hline $\mathrm{H}$ & -1.990062 & 0.032628 & 2.801838 \\
\hline $\mathrm{H}$ & 0.520595 & 1.930831 & 4.485583 \\
\hline $\mathrm{H}$ & 0.017095 & 0.158017 & -4.114289 \\
\hline $\mathrm{H}$ & 0.974606 & 1.403759 & -4.356643 \\
\hline 0 & 1.072779 & 0.017548 & 1.569997 \\
\hline 0 & 3.589444 & -1.290324 & 1.490481 \\
\hline $\mathrm{O}$ & 3.593102 & -1.409865 & -1.357696 \\
\hline 0 & 3.583385 & 1.417105 & 1.357907 \\
\hline $\mathrm{O}$ & 2.060299 & 2.479241 & 2.653458 \\
\hline 0 & 0.136489 & 3.951821 & 1.477372 \\
\hline
\end{tabular}




\begin{tabular}{|c|c|c|c|}
\hline 0 & -2.072246 & 2.866877 & 2.129819 \\
\hline $\mathrm{O}$ & -2.09029 & 2.492259 & -2.551555 \\
\hline $\mathrm{O}$ & -3.60675 & 1.346477 & 1.45514 \\
\hline $\mathrm{O}$ & -3.635546 & 1.402554 & -1.314784 \\
\hline O & -3.630876 & -1.415702 & 1.312299 \\
\hline $\mathrm{O}$ & -3.598407 & -1.358102 & -1.455662 \\
\hline $\mathrm{O}$ & -2.059041 & -2.872866 & -2.132845 \\
\hline $\mathrm{O}$ & -2.081917 & -2.503359 & 2.546604 \\
\hline $\mathrm{O}$ & -1.138087 & -1.597141 & 0.011164 \\
\hline O & -1.386312 & 0.039047 & 2.044678 \\
\hline $\mathrm{O}$ & -0.125422 & 1.454614 & 3.945441 \\
\hline $\mathrm{O}$ & 0.081004 & -1.18075 & 4.058998 \\
\hline O & 0.078891 & 1.176001 & -4.058979 \\
\hline $\mathrm{O}$ & -1.145313 & 1.590811 & -0.011466 \\
\hline $\mathrm{O}$ & 1.391331 & 1.981309 & -0.044332 \\
\hline $\mathrm{O}$ & 2.02378 & 2.421724 & -2.688355 \\
\hline $\mathrm{O}$ & -0.111515 & 3.884174 & -1.42597 \\
\hline O & 3.583718 & 1.296282 & -1.486145 \\
\hline 0 & -0.0967 & -3.889234 & 1.426772 \\
\hline O & 0.150253 & -3.955833 & -1.4765 \\
\hline 0 & 1.400125 & -1.982761 & 0.045736 \\
\hline O & 2.034972 & -2.421612 & 2.693517 \\
\hline $\mathrm{O}$ & 2.075781 & -2.480697 & -2.652441 \\
\hline 0 & -0.113481 & -1.460487 & -3.945742 \\
\hline 0 & -1.383812 & -0.047319 & -2.045442 \\
\hline $\mathrm{O}$ & 1.074265 & -0.019626 & -1.56898 \\
\hline $\mathrm{Zr}$ & -2.500614 & -0.005223 & -0.000456 \\
\hline $\mathrm{Zr}$ & 0.081512 & 1.827225 & 1.886096 \\
\hline $\mathrm{Zr}$ & 0.028968 & -1.781613 & 1.822797 \\
\hline $\mathrm{Zr}$ & 0.091352 & -1.832153 & -1.886524 \\
\hline $\mathrm{Zr}$ & 2.447949 & 0.000238 & 0.00102 \\
\hline $\mathrm{Zr}$ & 0.022216 & 1.776545 & -1.82209 \\
\hline $\mathrm{Zn}$ & 1.312752 & 3.901901 & -0.05979 \\
\hline $\mathrm{Zn}$ & -1.269132 & 3.692068 & 0.14533 \\
\hline $\mathrm{Zn}$ & -1.255372 & -3.697756 & -0.14411 \\
\hline $\mathrm{Zn}$ & 1.327163 & -3.903375 & $0.0602 s$ \\
\hline
\end{tabular}

\section{Reference}

1. Planas, N.; Mondloch, J. E.; Tussupbayev, S.; Borycz, J.; Gagliardi, L.; Hupp, J. T.; Farha, O. K.; Cramer, C. J., Defining the Proton Topology of the $\mathrm{Zr}_{6}$-Based MetalOrganic Framework NU-1000. J. Phys. Chem. Lett. 2014, 5, (21), 3716-3723. 